Um novo esquema upwind de alta resolução para equações de conservação não estacionárias dominadas por convecção

\author{
Laís Corrêa
}





\section{Um novo esquema upwind de alta resolução para equações de conservação não estacionárias dominadas por convecção}

\section{Laís Corrêa}

Orientador: Prof. Dr. Valdemir Garcia Ferreira

Dissertação apresentada ao Instituto de Ciências Matemáticas e de Computação - ICMC-USP, como parte dos requisitos para obtenção do título de Mestre em Ciências - Ciências de Computação e Matemática Computacional. VERSÃO REVISADA.

USP - São Carlos

Abril/2011 
Ficha catalográfica elaborada pela Biblioteca Prof. Achille Bassi e Seção Técnica de Informática, ICMC/USP, com os dados fornecidos pelo(a) autor(a)

\begin{tabular}{|c|c|}
\hline \multirow[t]{3}{*}{ C824n } & $\begin{array}{l}\text { Corrêa, Laís } \\
\text { Um novo esquema upwind de alta resolução para } \\
\text { equações de conservação não estacionárias dominadas } \\
\text { por conveção / Laís Corrêa; orientador Valdemir } \\
\text { Garcia Ferreira -- São Carlos, } 2011 . \\
\quad 128 \text { p. }\end{array}$ \\
\hline & $\begin{array}{l}\text { Dissertação (Mestrado - Programa de Pós-Graduação en } \\
\text { Ciências de Computação e Matemática Computacional) -- } \\
\text { Instituto de Ciências Matemáticas e de Computação, } \\
\text { Universidade de São Paulo, } 2011 .\end{array}$ \\
\hline & $\begin{array}{l}\text { 1. esquemas convectivos. 2. equações de Navier- } \\
\text { Stokes. 3. leis de conservação. 4. escoamentos com } \\
\text { superfícies livres móveis. 5. upwind. I. Garcia } \\
\text { Ferreira, Valdemir, orient. II. Título. }\end{array}$ \\
\hline
\end{tabular}


Dedico às pessoas que mais amo na minha vida: meus pais. 



\section{Agradecimentos}

A Deus, pelo dom da vida e por iluminar meu caminho ao longo deste trabalho.

Aos meus pais, Maria José e Antônio, pelo grande amor, paciência, compreensão e grande apoio ao longo de todos os meus estudos. Também aos meus demais familiares que sempre estiveram ao meu lado nesta caminhada pedindo a Deus que sempre guiasse meu caminho.

Ao meu amigo e orientador Prof. Dr. Valdemir Garcia Ferreira pela paciência, disponibilidade, apoio, confiança e, principalmente, pelos seus ensinamentos e orientações.

A minha orientadora em iniciação científica Profa. Dra. Magda Kimico Kaibara pelos seus ensinamentos e apoio.

A minha amiga Giseli Ap. Braz de Lima por toda ajuda, paciência, carinho e trabalho em equipe.

Aos meus amigos Patrícia, Josuel, Larissa, Rafael, Miguel e Ítalo por toda ajuda e compreensão e, principalmente, pelos bons momentos e risadas.

As minhas amigas Letrícia e Josiane por toda compreensão, paciência e carinho.

Aos funcionários do ICMC-USP por toda dedicação, em especial ao meu amigo Leonardo Martinussi por toda sua paciência e ajuda.

A todos os professores do LCAD-ICMC/USP pelos ensinamentos e reflexões que me auxiliaram na construção deste trabalho.

A FAPESP, pelo suporte financeiro concedido para realização do meu projeto de pesquisa.

Finalmente, agradeço a todos que direta ou indiretamente contribuíram para a realização deste trabalho. 


\section{Resumo}

Neste trabalho apresenta-se um novo esquema prático tipo upwind de alta resolução, denominado EPUS (Eight-degree Polynomial Upwind Scheme), para resolver numericamente equações de conservação não estacionárias dominadas por convecção. O esquema é baseado no critério de estabilidade TVD e é implementado no contexto do método das diferenças finitas. O desempenho do esquema é investigado na resolução de sistemas hiperbólicos de leis de conservação e escoamentos incompressíveis complexos com superfícies livres. Os resultados numéricos mostraram boa concordância com outros resultados numéricos e dados experimentais existentes.

Palavras-chave: esquemas convectivos, equações de Navier-Stokes, leis de conservação, escoamentos com superfícies livres móveis, upwinding, modelo $\kappa-\varepsilon$. 


\section{Abstract}

In this work a new practical high resolution upwinding scheme, called EPUS (Eight-degree Polynomial Upwind Scheme), for the numerical solution of transient convection-dominated conservation equations is presented. The scheme is based on TVD stability criterion and is implemented in the context of the finite difference methodology. The performance of the scheme is investigated by solving hyperbolic systems of conservation laws and complex incompressible flows with free surfaces. The numerical results displayed good agreement with other existing numerical and experimental data.

Key-words: convective schemes, Navier-Stokes equations, conservation laws, free surface flows, upwinding, $\kappa-\varepsilon$ model. 


\section{Sumário}

1 Introdução 1

2 Modelagem Matemática $\quad 5$

2.1 Leis de Conservação 1D . . . . . . . . . . . . . . . . . . 5

2.1.1 Equação de Advecção 1D . . . . . . . . . . . . . . . . . . . . 5

2.1 .2 Equação de Burgers 1D . . . . . . . . . . . . . . . . 6

2.1 .3 Equações de Euler 1D . . . . . . . . . . . . . . . . . . . . 7

2.2 Leis de Conservação 2D . . . . . . . . . . . . . . . . . . 8

2.2.1 Equações de Euler 2D . . . . . . . . . . . . . . . . . . . . . . . 8

2.2 .2 Equações de Águas Rasas 2D . . . . . . . . . . . . . . . . . . . . . 9

2.3 Equações de Navier-Stokes . . . . . . . . . . . . . . . . . . . . . . 9

2.4 Equações Médias de Reynolds . . . . . . . . . . . . . . . . . . . . . 11

2.5 Modelagem $\kappa-\varepsilon$ da Turbulência . . . . . . . . . . . . . . . . . . . 12

3 Desenvolvimento de Esquemas Upwind $\quad 15$

3.1 Aproximação dos Termos Convectivos . . . . . . . . . . . . . . . . . . 15

3.2 Variáveis Normalizadas . . . . . . . . . . . . . . . . . . . . 16

3.3 Limitador de Fluxo . . . . . . . . . . . . . . . . . . . . . . 18

3.4 Critério de Limitação CBC . . . . . . . . . . . . . . . . . . . . . . . 19

3.5 Restrições TVD . . . . . . . . . . . . . . . . . . . . . . 19

3.6 Alguns Esquemas de Alta Resolução . . . . . . . . . . . . . . . . . . . 22

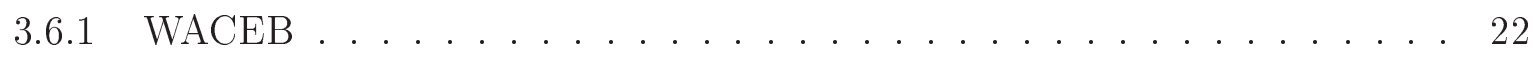

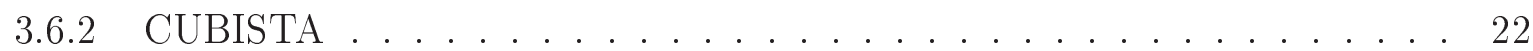

3.6 .3 Superbee . . . . . . . . . . . . . . . . . . . 23

3.6 .4 van Leer . . . . . . . . . . . . . . . . . . . . . . 23

3.6.5 Monotonized Central-difference $(\mathrm{MC}) \ldots \ldots \ldots . \ldots . \ldots . \ldots 24$

3.6 .6 ADBQUICKEST . . . . . . . . . . . . . . . . 24 


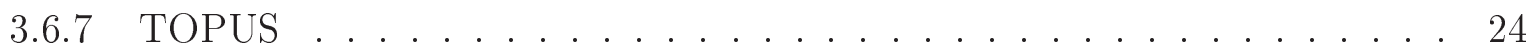

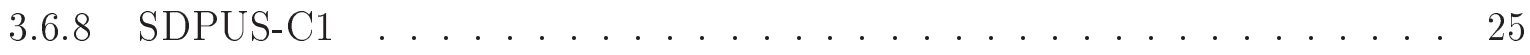

3.7 Estimativa de Erro . . . . . . . . . . . . . . . . . . 25

$\begin{array}{llr}4 & \text { O Esquema EPUS } & 27\end{array}$

4.1 Derivação do Esquema EPUS . . . . . . . . . . . . . . . . . 27

4.2 Limitador de Fluxo do Esquema EPUS . . . . . . . . . . . . . . . . . . . . . 29

4.3 Considerações Relevantes sobre o Esquema EPUS . . . . . . . . . . . . . . 30

5 Modelagem Computacional

5.1 Discretização das Leis de Conservação 1D . . . . . . . . . . . . . . . 33

5.1 .1 Equação de Adveç̧ão . . . . . . . . . . . . . . . . . . 34

5.1 .2 Equação de Burgers . . . . . . . . . . . . . . . . . . . . . 34

5.1 .3 Sistemas Hiperbólicos: Euler e Águas Rasas . . . . . . . . . . . . . . . . 34

5.2 Discretização das Leis de Conservação 2D . . . . . . . . . . . . . . 35

5.3 Discretização das Equações de Navier-Stokes . . . . . . . . . . . . . . . . 35

5.3 .1 Malha Computacional . . . . . . . . . . . . . . . 35

5.3.2 Discretização dos Termos Convectivos - Caso Laminar . . . . . . . . . . . 36

5.3.3 Discretização dos Termos Convectivos - Caso Turbulento . . . . . . . . . 40

5.3.4 Metodologia Computacional para as Equações de Navier-Stokes . . . . . 43

6 Resultados Numéricos 1D $\quad 47$

6.1 Equação Linear de Advecção . . . . . . . . . . . . . . . . . . . . . . . . 47

6.2 Equação Não-Linear de Burgers . . . . . . . . . . . . . . . . . . . . . 52

6.3 Equações Não-Lineares de Euler . . . . . . . . . . . . . . . . . . . . . . . . 59

7 Resultados Numéricos 2D e Axissimétricos $\quad 69$

7.1 Leis de Conservação 2D . . . . . . . . . . . . . . . . . . . . 69

7.1.1 Sistema Não-Linear de Euler . . . . . . . . . . . . . . . . . . . . 69

7.1 .2 Sistema Não-Linear de Águas Rasas . . . . . . . . . . . . . . . . . . . . 75

7.2 Escoamentos Incompressíveis Laminares 2D . . . . . . . . . . . . . . . 78

7.2.1 Colapso de uma Coluna de Fluido . . . . . . . . . . . . . . . . . . . 79

7.2.2 Jato Livre sobre uma Superfície Rígida Impermeável . . . . . . . . . . . 81

7.3 Escoamentos Incompressíveis Turbulentos 2D . . . . . . . . . . . . . . 83

7.4 Escoamentos Incompressíveis Laminares Axissimétricos . . . . . . . . . . . . 88

7.4.1 Ressalto Hidráulico Circular . . . . . . . . . . . . . . . . . . . 88

7.4.2 Experimento de Taylor . . . . . . . . . . . . . . . . . . . 91 
8 Resultados Numéricos 3D $\quad 95$

8.1 Colapso de um bloco de fluido . . . . . . . . . . . . . . . . . . . . 95

8.2 Ressalto Hidráulico Circular . . . . . . . . . . . . . . . . . . . . . . . 98

8.3 Jato Circular Oscilante . . . . . . . . . . . . . . . . . . 100

8.4 Jato Planar Oscilante . . . . . . . . . . . . . . . . . . . . . 105

9 Considerações Finais e Planos Futuros $\quad 107$

10 Produção Científica Associada $\quad 109$

10.1 Artigos e Resumos Publicados em Anais de Congressos . . . . . . . . . . . . . 109

10.2 Artigos Submetidos em Anais de Congressos . . . . . . . . . . . . . . . 113

10.3 Artigos Aceitos em Revistas Internacionais . . . . . . . . . . . . . . . . . 113

10.4 Cursos de Curta Duração Ministrados . . . . . . . . . . . . . . . . 114

$\begin{array}{ll}\text { A EPUS e a Propriedade TVD } & 115\end{array}$

B Esquema Desenvolvido Sem Parâmetro Livre $\quad 117$

$\begin{array}{ll}\text { C Software CLAWPACK } & 121\end{array}$

$\begin{array}{ll}\text { Referências Bibliográficas } & 123\end{array}$ 


\section{Lista de Figuras}

3.1 efeitos de difusão numérica: comparação entre o perfil característico de esquemas de primeira, segunda e alta ordem de precisão. . . . . . . . . . . . . . 16

3.2 posição dos nós computacionais $\mathbf{D}, \mathbf{U}$ e $\mathbf{R}$. . . . . . . . . . . . . . 16

3.3 diagrama de variáveis normalizadas mostrando os esquemas QUICK, diferença central e FOU. . . . . . . . . . . . . . . . . . . . . . . 17

3.4 região $\mathrm{CBC}$ em variáveis normalizadas. . . . . . . . . . . . . . . . . 19

3.5 região TVD para o limitador de fluxo. . . . . . . . . . . . . . . . . . . 21

3.6 região TVD em variáveis normalizadas. . . . . . . . . . . . . . . . . . 22

4.1 EPUS contido na região TVD para $\lambda=16$ e $\lambda=95$ (a) em variáveis normalizadas e (b) na forma de limitador de fluxo. . . . . . . . . . . . . . . . . . . 32

5.1 localização das componentes da velocidade numa típica célula computacional de uma malha deslocada 3D. . . . . . . . . . . . . . . . . . . . 36

5.2 representação esquemática para aproximação dos termos convectivos, em que $P$ é o ponto de discretização e $\phi_{f}$ e $\phi_{g}$ são as velocidades de convecção nas faces $f$ e $g$, respectivamente. . . . . . . . . . . . . . . . . 37

6.1 comparação para o Teste-1 - Problema Suave de alguns valores do parâmetro livre $\lambda$ para o caso de advecção de um escalar. . . . . . . . . . . . . . . . . . 48

6.2 comparação para o Teste-1 - Problema Não-Suave de alguns valores do parâmetro livre $\lambda$ para o caso de advecção de um escalar. . . . . . . . . . . . . . 49

6.3 análise da TV feita em Teste-2: (a) comparação entre as soluções exata e numéricas e (b) comportamento da TV do esquema EPUS em diferentes malhas. 50

6.4 comparação para o Teste-4 - Problema Suave de alguns valores do parâmetro livre $\lambda$ para o caso não linear. . . . . . . . . . . . . . . . . 53

6.5 comparação para o Teste-4 - Problema Não-Suave de alguns valores do parâmetro livre $\lambda$ para o caso não linear. . . . . . . . . . . . . . . . . . 54 
6.6 regiões de ampliação da Figura 6.5 . . . . . . . . . . . . . . . . 55

6.7 análise da TV feita no Teste-5: (a) comparação entre as soluções exata e numéricas e (b) comportamento da TV do esquema EPUS em diferentes malhas. . . . . 56

6.8 ilustração dos resultados obtidos sem e com correção de entropia para o Teste-7. 59

6.9 soluções de referência e numéricas para a densidade no problema Two Interacting Blast Waves. . . . . . . . . . . . . . . . . . . . . . 61

6.10 soluções de referência e numéricas para a energia total no problema Two Interacting Blast Waves. . . . . . . . . . . . . . . . . . . . .

6.11 soluções de referência e numéricas para a velocidade no problema Two Interacting Blast Waves. . . . . . . . . . . . . . . . . . . 63

6.12 modelo do tubo de choque de Sod em $t=0$.

6.13 soluções de referência e numéricas para a densidade no problema do tubo de choque de Sod.

6.14 soluções de referência e numéricas para a energia interna no problema do tubo de choque de Sod. . . . . . . . . . . . . . . . . . 67 67

7.1 perfil da densidade para o problema Four-shocks problem (Teste-1) . . . . . . 71

7.2 comportamento da densidade sobre a reta $y=x$ para o problema Four-shocks problem (Teste-1) . . . . . . . . . . . . . . . . . 72

7.3 perfil da densidade numa malha refinada para o Four-shocks problem (Teste-1). 73

7.4 continuação da Figura 7.3. . . . . . . . . . . . . . . . . . . . . 74

7.5 perfil da densidade numa malha refinada para o Four-contacts problem (Teste-2). 75

7.6 radial dam-break problem: comportamento da altura da porção de fluido nos instantes (a) $t=0$ e (b) $t=0.25$ (figura extraída de LeVeque [31]) . . . . . . . 76

7.7 perfil da altura para o problema radial dam-break problem (Teste-3). . . . . . . 77

7.8 comportamento da altura sobre a reta $y=0$ para o problema radial dam-break problem (ver Teste-3) . . . . . . . . . . . . . . . . . . 78

7.9 regiões de ampliação da Figura 7.8. . . . . . . . . . . . . . . . . 78

7.10 ilustração esquemática do problema de colapso de uma coluna de fluido. . . . . . 79

7.11 comparação das soluções para o problema do colapso de uma coluna de fluido. 80

7.12 contorno da pressão para o problema do colapso de uma coluna de fluido em diferentes tempos. . . . . . . . . . . . . . . . . . 80

7.13 contorno da velocidade na direção $x$ para o problema do colapso de uma coluna de fluido em diferentes tempos.

7.14 contorno da velocidade na direção y para o problema do colapso de uma coluna de fluido em diferentes tempos.

7.15 ilustração esquemática de um jato livre incidindo perpendicularmente sobre uma superfície rígida impermeável. 
7.16 comparação das soluções para o problema do jato livre sobre uma superfície rígida impermeável. . . . . . . . . . . . . . . . . . . . . . 83

7.17 contorno da pressão para o problema do jato livre sobre uma superfície rígida impermeável em diferentes tempos.

7.18 contorno da velocidade na direção $x$ para o problema do jato livre sobre uma superfície rígida impermeável em diferentes tempos.

7.19 contorno da velocidade na direção y para o problema do jato livre sobre uma superfície rígida impermeável em diferentes tempos. . . . . . . . . . . . . . 85

7.20 comparação das soluções para o problema do jato livre sobre uma superfície rígida impermeável (caso turbulento).

7.21 contorno da pressão para o problema do jato livre sobre uma superfície rígida impermeável (caso turbulento).

7.22 contorno da velocidade na direção $x$ para o problema do jato livre sobre uma superfície rígida impermeável (caso turbulento).

7.23 contorno da velocidade na direção y para o problema do jato livre sobre uma superfície rígida impermeável (caso turbulento).

7.24 ilustração esquemática do ressalto hidráulico circular.

7.25 comparação das soluções para o problema do ressalto hidráulico circular.

7.26 comparação das soluções para o problema do ressalto hidráulico circular utilizando diferentes valores para $\delta t$.

7.27 ilustração do ressalto hidráulico circular: resultados (a) experimental e (b) simulado pelo esquema EPUS. . . . . . . . . . . . . . . . . . . . 9 90

7.28 ilustração esquemática do experimento de Taylor. . . . . . . . . . . . . . . . 92

7.29 ilustração do experimento de Taylor: resultados (a) experimental e (b) simulado pelo esquema EPUS em diferentes tempos. . . . . . . . . . . . . . . . . . . 93

7.30 ilustração dos resultados obtidos pelo esquema EPUS (corte transversal em (b) para mostrar a estrutura vortical).

7.31 experimento de Taylor simulado pelo esquema EPUS: perfis de (a) pressão, (b) velocidade na direção $x$ e (c) velocidade na direção $y$ no tempo $t=10 s$. . . . . 94

8.1 ilustração esquemática para o problema do colapso de um bloco de fluido. . . . . 96

8.2 comparação das soluções para o problema do colapso de um bloco de fluido. . . 96

8.3 campo da pressão para o problema do colapso de um bloco de fluido em diferentes tempos.

8.4 campo da velocidade na direção $x$ para o problema do colapso de um bloco de fluido em diferentes tempos.

8.5 campo da velocidade na direção $z$ para o problema do colapso de um bloco de fluido em diferentes tempos. 
8.6 ilustração do ressalto hidráulico circular para $R e=250$ : resultados (a) experimental e (b) simulado pelo esquema EPUS. . . . . . . . . . . . . . . . . . . . . 99

8.7 ilustração do ressalto hidráulico circular para $R e=1000$ : resultados (a) experimental e (b) simulado pelo esquema EPUS. . . . . . . . . . . . . . . . 100

8.8 ilustração esquemática para problema do jato circular oscilante. . . . . . . . . 101

8.9 ilustração dos resultados obtidos pelo EPUS para o fenômeno do jato circular oscilante $($ Teste-4) . . . . . . . . . . . . . . . . . . . 102

8.10 ilustração do jato circular oscilante para o Teste-5: resultados (a) experimental e (b) simulado com o esquema EPUS. . . . . . . . . . . . . . . . . . . . . . 104

8.11 ilustração esquemática para o problema do jato planar oscilante. . . . . . . . . 105

8.12 ilustração dos resultados obtidos pelo EPUS para o fenômeno do jato planar oscilante no Teste-6. . . . . . . . . . . . . . . . . . . . 106 


\section{Lista de Tabelas}

6.1 erros relativos obtidos pelo EPUS para o Teste-1 - Problema Suave. . . . . . 48

6.2 erros relativos obtidos pelo EPUS para o Teste-1 - Problema Não-Suave. . . 50

6.3 erros relativos nas normas $L_{1}$ e $L_{\infty}$ e ordem de convergência $(\tilde{p})$ calculados para o Teste-3 utilizando como aproximação temporal Euler explícito e Runge-Kutta TVD de terceira ordem. . . . . . . . . . . . . . . . . . . 51

6.4 tempo de CPU calculado para o Teste-3. . . . . . . . . . . . . . . . 52

6.5 erros relativos obtidos pelo EPUS para o Teste-4 - Problema Suave. . . . . . 53

6.6 erros relativos obtidos pelo EPUS para o Teste-4 - Problema Não-Suave. . . 55

6.7 erros relativos nas normas $L_{1}$ e $L_{\infty}$ e estimativa para ordem de convergência $(\tilde{p})$ calculados para o Teste-6. . . . . . . . . . . . . . . 57

6.8 resultados obtidos para o Teste-8: erro relativo $E_{h}$ e estimativa para a ordem de convergência $\tilde{p}$, ambos obtidos na norma $L_{1}$, e tempo de CPU em cada malha. 64

7.1 ordem de convergência $\tilde{p}$ na norma $L_{1}$ para o Teste-1 em diferentes tempos. . . 72

7.2 comparação dos valores obtidos para o raio do ressalto hidráulico circular. . . . . 91 
CAPÍTULO

\section{1}

\section{Introdução}

Esquemas de convecção de alta resolução são bastante empregados hoje em dia para resolver problemas em dinâmica dos fluidos, especialmente para a classe de problemas de escoamentos incompressíveis não estacionários envolvendo superfícies livres móveis [12, 58] a altos valores do número de Reynolds. Embora muitos pesquisadores têm atentado para tais problemas, não é possível até o presente caracterizá-los por completo. Conseguir soluções numéricas representativas para essa classe de problemas tem sido difícil em virtude da forte influência dos termos convectivos (em geral não lineares) nas equações de transporte. Consequentemente, a escolha do método numérico que leva em conta a velocidade de convecção local do escoamento (usualmente conhecido como esquema de convecção upwind - vide [34] ou [38]) tem sido uma das principais preocupações da comunidade científica moderna em dinâmica dos fluidos computacional, uma vez que esse tipo de aproximação afeta sobre maneira a estabilidade e a precisão da solução.

Por exemplo, ao se usar esquemas upwind de primeira ordem, tais como FOU (First Order Upwind) [13] e o método Híbrido [42, 53], a solução computada sofre suavização, pois esses esquemas introduzem viscosidade numérica amortecendo as oscilações (físicas e não físicas). Por outro lado, ao se utilizar esquemas clássicos de alta ordem (maior ou igual a 2), tais como QUICK (Quadratic Upstream Interpolation for Convective Kinematics) [29] ou Lax-Wendroff [28], a solução numérica geralmente apresenta oscilações não físicas, principalmente em descontinuidades (choques) e regiões de altos gradientes, onde na maioria das vezes surgem instabilidades numéricas inevitáveis. De fato, pelo teorema de Godunov [48] nenhum esquema linear de ordem maior que 1 é monotônico (livre de oscilações). Isso tem motivado o desenvolvimento de esquemas convectivos upwind não lineares de alta ordem, os quais ajustam a ordem de precisão de acordo com a solução local de modo a manter um comportamento limitado.

Nas últimas décadas muitas tentativas têm sido feitas para derivar o esquema convectivo 
upwind de alta resolução "perfeito", isto é, aquele que satisfaz as seguintes propriedades: estabilidade, captura de descontinuidades sem a presença de oscilações numéricas (oscilações limitadas), simplicidade, economia computacional, convergência e a resolução de uma variedade de fenômenos complexos. Entre elas destacam-se a proposta do esquema HLPA (Hybrid-Linear Parabolic Approximation) de Zhu [69], do SMART (Sharp and Monotonic Algorithm for Realistic Transport) por Gaskell e Lau [21], do WACEB (Weighted-Average Coefficient Ensuring Boundedness) por Song et al. [37], do VONOS (Variable-Order Non-Oscillatory Scheme) de Varonos e Bergeles [63], do CUBISTA (Convergent and Universally Bounded Interpolation Scheme for the Treatment of Advection) de Alves et al. [1], do MUSCL (Monotone Upstream Scheme for Conservation Laws) por van Leer [61, 62] e, mais recentemente, a proposta do ADBQUICKEST por Ferreira et al. [19] e TOPUS por Queiroz [15]. Tais esquemas vêm sendo muito utilizados numa variedade de aplicações práticas. No entanto, muitas dificuldades ainda persistem quando se deseja simular escoamentos incompressíveis em regime transitório, principalmente os efeitos da turbulência, pois, nesse caso, os escoamentos tornam-se intrinsicamente transientes, tridimensionais e com uma variedade de escalas envolvidas no movimento.

Portanto, a necessidade de um esquema de convecção upwind simples, eficiente e robusto para aproximar termos convectivos não lineares de leis de conservação e equações transientes da dinâmica dos fluidos continua a estimular a pesquisa na área de CFD. Esta é a principal motivação para o estudo apresentado neste trabalho. Outra motivação para o desenvolvimento de um esquema upwind está no desejo da autora em desenvolver uma técnica numérica capaz de simular uma variedade de problemas de interesse prático.

Há também outra classe de esquemas de alta resolução sofisticados chamados ENO (Essentially Non-Oscillatory) [50] e seus relacionados WENO (Weighted ENO) [4, 26], que são apropriados para capturar descontinuidades e pontos extremos com alta ordem de precisão. Nesses esquemas, alta ordem é alcançada usando polinômios interpoladores (por partes) de graus elevados que não garantem resultados não oscilatórios. Seus compromissos são relaxar as condições TVD/CBC e, ao invés de considerar moléculas computacionais fixas, escolher moléculas computacionais variáveis. O preço a ser pago por esse procedimento é, além da dificuldade de implementação, o número muito elevado de operações aritméticas requeridas a cada passo no tempo, sendo isso mais uma motivação para o presente trabalho de mestrado. A diferença entre ENO/WENO e esquemas TVD/CBC é que os primeiros têm a propriedade de reter a mesma ordem de precisão espacial em todo o domínio de solução, inclusive nas vizinhanças de descontinuidades e pontos extremos, enquanto que os esquemas TVD/CBC oferecem primeira ordem de precisão nessas regiões críticas.

No contexto do método das diferenças finitas (ver, por exemplo, [33]) e fazendo o uso de variáveis normalizadas e/ou limitadores de fluxo e dos critérios de limitação CBC e/ou TVD, apresenta-se no presente trabalho de mestrado o novo esquema de convecção EPUS, que tem se mostrado simples, eficiente e robusto. O foco principal deste trabalho é, usando o esquema 
EPUS, a simulação numérica de problemas de escoamentos de fluidos não estacionários para os casos compressível e incompressível.

A contribuição da pesquisa científica aqui apresentada é inovadora, pois visa a análise, a implementação computacional e a construção de um novo método numérico capaz de simular uma variedade de problemas de escoamentos de fluidos, permitindo, no contexto atual, melhorias nas predições de problemas de engenharia.

Vale mencionar que o presente projeto de pesquisa é financiado pela FAPESP (processo $n^{\circ}$ 2008/07367-9) e contempla objetivos do projeto temático Solução numérica das equações de Navier-Stokes - processo $n^{o}$ 2000/03385-0, já desenvolvido no ICMC-USP com o apoio da FAPESP. Contempla também um dos objetivos do projeto temático Mecânica dos fluidos não estacionária: aplicações em aeronáutica e em reologia - processo no 2004/16064-9, financiado pela FAPESP e desenvolvido em conjunto com ICMC-USP, EESC-USP e IAE-CTA.

O presente projeto de pesquisa contempla os seguintes objetivos, a saber:

- Desenvolver e implementar um novo esquema convectivo de alta resolução para resolver problemas não estacionários em dinâmica dos fluidos;

- Corroborar a asserção de Arora e Roe [2] de que esquemas TVD, associados ao avanço temporal explícito de primeira ordem, têm méritos em problemas transientes;

- Dispor de códigos 2D/3D para simular numericamente problemas de escoamentos incompressíveis com superfícies livres móveis a uma ampla faixa do número de Reynolds;

- Fornecer à literatura novas soluções numéricos de EDPs não lineares que possam auxiliar pesquisadores da área de CFD.

Os demais capítulos deste texto de dissertação estão dispostos como segue:

- No Capítulo 2 apresentam-se as equações básicas utilizadas neste trabalho de mestrado, que são EDPs que aparecem com frequência em CFD;

- O Capítulo 3 é reservado para uma apresentação da base teórica para o desenvolvimento de esquemas upwind de alta resolução. Também apresentam-se alguns esquemas de alta resolução presentes na literatura, os quais são utilizados neste trabalho para comparações. Apresentam-se ainda estimativas para o erro cometido, bem como o cálculo da ordem de convergência observada;

- No Capítulo 4 descreve-se o desenvolvimento do novo esquema upwind polinomial de alta resolução EPUS, bem como faz-se algumas considerações relevantes sobre esta nova ferramenta numérica;

- O Capítulo 5 contém a modelagem computacional utilizada para a resolução numérica das leis de conservação e das equações de Navier-Stokes, bem como suas discretizações destas equações, dando-se ênfase à discretização dos termos convectivos. Descreve-se ainda a metodologia numérica e computacional utilizada para a simulação dos escoamentos incompressíveis laminares e turbulentos;

- No Capítulo 6 apresentam-se resultados numéricos para leis de conservação hiperbólicas 
1D obtidos pelo esquema EPUS (descrito no Capítulo 4) e também pelos demais esquemas de alta resolução apresentados no Capítulo 3;

- No Capítulo 7 apresentam-se resultados numéricos para leis de conservação hiperbólicas 2D, bem como para as equações de Navier-Stokes 2D (ambos os casos laminar e turbulento) e axissimétricas;

- No Capítulo 8 apresentam-se resultados numéricos de escoamentos incompressíveis laminares 3D, os quais são modelados pelas equações instantâneas de Navier-Stokes;

- No Capítulo 9 apresentam-se as considerações finais e os planos futuros;

- Por fim, no Capítulo 10 lista-se a produção científica associada ao presente trabalho de mestrado. 
CAPÍTULO

\section{Modelagem Matemática}

Neste capítulo descreve-se a formulação matemática utilizada ao longo deste projeto de mestrado, ou seja, as EDPs básicas que aparecem com frequência em CFD, a saber: leis de conservação 1D (equação linear de advecção, equação de Burgers e equações de Euler), leis de conservação 2D (sistemas hiperbólicos de Euler e águas rasas), equações instantâneas e médias de Navier-Stokes e modelagem $\kappa-\varepsilon$ da turbulência. O estudo destas equações é de fundamental importância para o entendimento do problema a ser modelado, bem como para sua implementação computacional.

\subsection{Leis de Conservação 1D}

Equações diferencias parciais podem ser usadas para modelar uma variedade de fenômenos da ciência e da engenharia (ver, por exemplo, [32]). Em mecânica dos fluidos, estas equações, também denominadas leis de conservação, são escritas em sua forma conservativa como

$$
\frac{\partial \phi}{\partial t}+\frac{\partial F(\phi)}{\partial x}=0
$$

em que $\phi=\phi(x, t)$ é o vetor das quantidades conservadas e $F(\phi)=F(\phi(x, t))$ é o vetor que representa as funções fluxo. Apresenta-se nesta seção três leis de conservação 1D, a saber: equação linear de advecção, equação de Burgers e equações de Euler.

\subsubsection{Equação de Advecção 1D}

A equação linear de advecção 1D é um dos modelos mais simples de EDPs hiperbólicas, sendo muito utilizada para o estudo de métodos numéricos em leis de conservação. Ela modela o transporte de escalares através da Eq. (2.1) (ver, por exemplo, [32] e [59]), considerando os 
vetores da propriedade conservada e da função fluxo dados, respectivamente, por

$$
\begin{gathered}
\phi=u, \\
F(u)=a u,
\end{gathered}
$$

sendo $a>0$ (constante) a velocidade de advecção da propriedade $u=u(x, t)$ no tempo $t$. As condições inicias e de contorno consideradas são, respectivamente,

$$
\begin{gathered}
u(x, 0)=u_{0}(x), \\
u\left(x_{L}, t\right)=u_{L}, \quad u\left(x_{R}, t\right)=u_{R},
\end{gathered}
$$

com $u_{L}$ e $u_{R}$ contantes, em que $x \in\left[x_{L}, x_{R}\right]$. A solução exata para este problema linear é dada por (ver LeVeque [32])

$$
u(x, t)=u_{0}(x-a t)
$$

A equação (2.6) mostra que a solução para este problema é obtida a partir de translações (advecções) uniformes da condição inicial com velocidade constante $a$. Isso se deve ao fato de que suas curvas características são dadas por $x$ - at, ou seja, são semi-retas paralelas (ver [32]).

\subsubsection{Equação de Burgers 1D}

A equação não linear de Burgers modela uma variedade de problemas em dinâmica dos fluidos (ver, por exemplo, [67]), sendo propensa à formação de choques, mesmo nos casos de dados iniciais suaves. Ela serve, por exemplo, como um modelo simplificado para o entendimento da turbulência, combinando convecção não linear e difusão linear, o que a leva, atualmente, a ser também muito utilizada no desenvolvimento de métodos numéricos (ver [32]).

A equação de Burgers é uma lei de conservação com fluxo quadrático, dada, em sua forma não viscosa, pela Eq. (2.1) com vetores da variável conservada e da função fluxo dados, respectivamente, por

$$
\begin{gathered}
\phi=u, \\
F(u)=\frac{1}{2} u^{2} .
\end{gathered}
$$


Na forma quase-linear, a equação de Burgers inviscida é dada por

$$
\frac{\partial u}{\partial t}+u \frac{\partial u}{\partial x}=0
$$

onde é possível observar que esta lei de conservação representa o transporte da propriedade $u$ com velocidade $u$, assemelhando-se à equação de advecção, a menos do fato de que agora temos uma velocidade de convecção não constante $u$. Sendo assim, as características não mais serão retas paralelas, podendo, em algum momento, se interceptarem, levando à formação de descontinuidades (choques), fenômeno característico de problemas não lineares.

As condições iniciais e de contorno adotadas na resolução desta EDP são dadas, respectivamente, por

$$
\begin{gathered}
u(x, 0)=u_{0}(x), \quad x \in\left[x_{L}, x_{R}\right], \\
u\left(x_{L}, t\right)=f\left(x_{L}, t\right), \quad u\left(x_{R}, t\right)=g\left(x_{R}, t\right) .
\end{gathered}
$$

\subsubsection{Equações de Euler 1D}

Estas EDPs constituem um sistema hiperbólico não linear de leis de conservação que modelam a dinâmica de um material compressível, tais como gases (ver [59]). Os problemas mais relevantes modelados por estas equações são os de tubo de choque, em que se estuda a interação entre diferentes gases.

Este sistema de equações é dado pela Eq. (2.1), sendo agora os vetores das quantidades conservadas e das funções fluxos dados, respectivamente, por

$$
\begin{gathered}
\phi=(\rho, \rho u, E)^{T}, \\
F(\phi)=\left(\rho u, \rho u^{2}+p, u(E+p)\right)^{T},
\end{gathered}
$$

em que as variáveis conservadas $\rho, u, \rho u, E$ e $p$ são, respectivamente, a massa específica, a velocidade, a quantidade de movimento, a energia total e a pressão. Para fechar este sistema, considera-se também a equação do gás ideal

$$
p=(\gamma-1)\left(E-\frac{1}{2} \rho u^{2}\right)
$$

sendo $\gamma=1.4$ a razão do calor específico. Ainda, tem-se que a energia interna e é dada por

$$
e=\frac{p}{(\gamma-1) \rho}
$$


A condição inicial considerada para a resolução deste sistema é dada por

$$
\left(\rho_{0}, u_{0}, p_{0}\right)^{T}=\left\{\begin{array}{ll}
\left(\rho_{L}, u_{L}, p_{L}\right)^{T}, & x \leq x_{0}, \\
\left(\rho_{R}, u_{R}, p_{R}\right)^{T}, & x>x_{0},
\end{array} \quad x \in\left[x_{L}, x_{R}\right] .\right.
$$

A condição de contorno adotada é a extrapolação de ordem zero (para mais detalhes, ver [32]).

\subsection{Leis de Conservação 2D}

Para o caso de problemas em geometria 2D, as leis de conservação consideradas neste trabalho são dadas por

$$
\frac{\partial \phi}{\partial t}+\frac{\partial F(\phi)}{\partial x}+\frac{\partial G(\phi)}{\partial y}=0
$$

em que $\phi=\phi(x, y, t)$ representa o vetor das variáveis conservadas, e $F(\phi)=F(\phi(x, y, t))$ e $G(\phi)=G(\phi(x, y, t))$ são os vetores das funções fluxo nas direções $x$ e $y$, respectivamente. Nesta seção são apresentadas duas leis de conservação 2D, a saber: equações não lineares de Euler e equações não lineares de águas rasas.

\subsubsection{Equações de Euler 2D}

As equações de Euler 2D, as quais modelam principalmente a dinâmica em gases, são dadas pela Eq. (2.17) com vetores das variáveis conservadas e das funções fluxos dados por

$$
\begin{gathered}
\phi=(\rho, \rho u, \rho v, E)^{T}, \\
F(\phi)=\left(\rho u, \rho u^{2}+p, \rho u v, u(E+p)\right)^{T}, \\
G(\phi)=\left(\rho v, \rho u v, \rho v^{2}+p, v(E+p)\right)^{T},
\end{gathered}
$$

em que $(u, v)^{T}$ representa o vetor velocidade. Para fechar este sistema, considera-se a equação do gás ideal

$$
p=(\gamma-1)\left(E-\frac{1}{2} \rho\left(u^{2}+v^{2}\right)\right)
$$

Estas equações são definidas neste trabalho em um domínio quadrado, o qual é dividido em quatro quadrantes pelas linhas $x=x_{0}$ e $y=y_{0}$. Esses quadrantes são definidos por estados 
iniciais constantes, de tal forma que

$$
\left(\rho_{0}, u_{0}, v_{0}, p_{0}\right)^{T}= \begin{cases}\left(\rho_{1}, u_{1}, v_{1}, p_{1}\right)^{T}, & \text { se } x>x_{0} \text { e } y>y_{0}, \\ \left(\rho_{2}, u_{2}, v_{2}, p_{2}\right)^{T}, & \text { se } x<x_{0} \text { e } y>y_{0}, \\ \left(\rho_{3}, u_{3}, v_{3}, p_{3}\right)^{T}, & \text { se } x<x_{0} \text { e } y<y_{0}, \\ \left(\rho_{4}, u_{4}, v_{4}, p_{4}\right)^{T}, & \text { se } x>x_{0} \text { e } y<y_{0}\end{cases}
$$

No contorno, aplica-se a condição de extrapolação de ordem zero (ver LeVeque [32]).

\subsubsection{Equações de Águas Rasas 2D}

Estas equações modelam o movimento hidrostático de um fluido incompressível com superfície livre (no caso água) em um canal de largura unitária, com velocidade horizontal $u(x, t)$ e velocidade vertical desprezada (ver, por exemplo, [32]). O sistema hiperbólico não linear de águas rasas 2D é dado pela Eq. (2.17), com vetores das quantidades conservadas e das funções fluxos dados por

$$
\begin{gathered}
\phi=(h, h u, h v)^{T}, \\
F(\phi)=\left(h u, h u^{2}+\frac{1}{2} g h^{2}, h u v\right)^{T}, \\
G(\phi)=\left(h u, h u v, h v^{2}+\frac{1}{2} g h^{2}\right)^{T},
\end{gathered}
$$

em que $h=h(x, y, t)$ é a altura da camada de água (profundidade do fluido no canal) e $g=9.81 \mathrm{~m} / \mathrm{s}^{2}$ é a gravidade. Neste sistema, $(u, v)^{T}$ e $(h u, h v)^{T}$ representam, respectivamente, os vetores velocidade e vazão.

As condições inicias adotadas na resolução deste sistema são dadas por

$$
u(x, y, 0)=u_{0}(x, y), \quad v(x, y, 0)=v_{0}(x, y) \quad \text { e } \quad h(x, y, 0)=h_{0}(x, y)
$$

em que $x \in\left[x_{L}, x_{R}\right]$ e $y \in\left[y_{L}, y_{R}\right]$. As condições de contorno foram adotadas de acordo com LeVeque [32], como extrapolação de ordem zero.

\subsection{Equações de Navier-Stokes}

Para o caso em que o fluido é considerado um meio homogêneo incompressível (a massa específica não varia durante o seu movimento) e as propriedades de transporte são constantes, as equações matemáticas das leis físicas de conservação são as equações de Navier-Stokes e continuidade, dadas, na forma adimensional, em coordenadas cartesianas e na notação de Einstein, 
por

$$
\begin{aligned}
\frac{\partial u_{i}}{\partial t}+\frac{\partial\left(u_{i} u_{j}\right)}{\partial x_{j}} & =-\frac{\partial p}{\partial x_{i}}+\frac{1}{R e} \frac{\partial}{\partial x_{j}}\left(\frac{\partial u_{i}}{\partial x_{j}}\right)+\frac{1}{F r^{2}} g_{i}, \quad i=1,2,3 \\
\frac{\partial u_{i}}{\partial x_{i}} & =0
\end{aligned}
$$

em que $u_{i}$ são as componentes do campo de velocidade, $t$ é o tempo e $p$ é a pressão. Os parâmetros adimensionais são $R e=L_{0} U_{0} / \nu$ e $F r=U_{0} / \sqrt{L_{0} g}$, os quais representam, respectivamente, os números de Reynolds e Froude, e $g_{i}$ são as componentes do campo gravitacional. O parâmetro $\nu=\mu / \rho>0$ é o coeficiente de viscosidade cinemática molecular (constante) do fluido, em que $\mu$ é a viscosidade dinâmica, e $U_{0}$ e $L_{0}$ são escalas de velocidade e comprimento característicos, respectivamente.

No caso de problemas com simetria radial (axissimétricos), as equações de Navier-Stokes são escritas em coordenadas cilíndricas, dadas, na forma adimensional, por

$$
\begin{aligned}
\frac{\partial u}{\partial t}+\frac{1}{r} \frac{\partial(r u u)}{\partial r}+\frac{\partial(u v)}{\partial z} & =-\frac{\partial p}{\partial r}+\frac{1}{R e} \frac{\partial}{\partial z}\left(\frac{\partial u}{\partial z}-\frac{\partial v}{\partial r}\right)+\frac{g_{r}}{F r^{2}} \\
\frac{\partial v}{\partial t}+\frac{1}{r} \frac{\partial(r v u)}{\partial r}+\frac{\partial(v v)}{\partial z} & =-\frac{\partial p}{\partial z}+\frac{1}{R e} \frac{1}{r} \frac{\partial}{\partial r}\left(r\left(\frac{\partial u}{\partial z}-\frac{\partial v}{\partial r}\right)\right)+\frac{g_{z}}{F r^{2}} \\
\frac{1}{r} \frac{\partial(r u)}{\partial r}+\frac{\partial v}{\partial z} & =0
\end{aligned}
$$

em que $u=u(r, z, t)$ e $v=v(r, z, t)$ são, respectivamente, as componentes do vetor velocidade nas direções $r$ e $z$, e $g=\left(g_{r}, g_{z}\right)^{T}$ representa o campo gravitacional.

Para resolver as equações de Navier-Stokes (em coordenadas cartesianas e cilíndricas) é necessário impor condições de contorno, as quais devem ser escolhidas de modo a respeitar o comportamento físico da solução. Neste trabalho, as condições de contorno adotadas para resolver estas equações são descritas a seguir:

- Entrada do fluido (injetor): considera-se condição de contorno prescrita, dada por

$$
u_{n}=U_{0}, \quad u_{t}=0
$$

em que $u_{n}$ é a velocidade normal ao contorno e $u_{t}$ é a velocidade tangencial a ele.

- Saída do fluido (ejetor): considera-se que não há variação da velocidade na direção normal ao contorno, de forma que

$$
\frac{\partial u_{n}}{\partial n}=\frac{\partial u_{t}}{\partial n}=0 .
$$


- Contorno rígido: aqui considera-se dois casos, a saber:

- No-slip (sem escorregamento): o fluido possui velocidade nula no contorno, isto é,

$$
u_{n}=0, \quad u_{t}=0
$$

- Free-slip (com escorregamento): não há perda friccional no contorno, de forma que

$$
u_{n}=0, \quad \frac{\partial u_{t}}{\partial n}=0
$$

- Superfície livre: considera-se que o fluido está imerso num ambiente inerte, de forma que

$$
\begin{aligned}
n \cdot(\sigma \cdot n) & =p_{\text {ext }}, \\
m_{1} \cdot(\sigma \cdot n) & =0, \\
m_{2} \cdot(\sigma \cdot n) & =0,
\end{aligned}
$$

em que $p_{\text {ext }}$ é a pressão externa (atmosférica), a qual foi assumida nula neste trabalho, e o tensor das tensões totais é dado, para os casos $2 \mathrm{D}$ e $3 \mathrm{D}$, por

$$
\sigma=-p I+2 \mu\left(\nabla u+(\nabla u)^{T}\right)
$$

e para o caso axissimétrico por

$$
\sigma=-p I+\frac{1}{R e}\left(\nabla u+(\nabla u)^{T}\right)
$$

sendo $I$ o tensor identidade. No caso $3 \mathrm{D}$, consideram-se os vetores tangenciais à superfície livre $m_{1}=\left(m_{1 x}, m_{1 y}, m_{1 z}\right)$ e $m_{2}=\left(m_{2 x}, m_{2 y}, m_{2 z}\right)$ e $n=\left(n_{x}, n_{y}, n_{z}\right)$ o vetor normal externo à superfície (no caso 2D tem-se $m_{1}=\left(m_{1 x}, m_{1 y}\right)$ e $n=\left(n_{x}, n_{y}\right)$ ). No caso axissimétrico, considera-se $m_{1}=\left(-n_{z}, 0, n_{r}\right)$ e $n=\left(n_{r}, 0, n_{z}\right)$.

\subsection{Equações Médias de Reynolds}

Para a simulação dos efeitos da turbulência, as equações de conservação instantâneas (2.26) e (2.27) são transformadas nas equações médias de Reynolds, da forma

$$
\begin{gathered}
\frac{\partial \bar{u}_{i}}{\partial t}+\frac{\partial\left(\bar{u}_{i} \bar{u}_{j}\right)}{\partial x_{j}}=-\frac{\partial \bar{p}}{\partial x_{i}}+\frac{1}{R_{e}} \frac{\partial}{\partial x_{j}}\left(\frac{\partial \bar{u}_{i}}{\partial x_{j}}\right)+\frac{1}{F r^{2}} g_{i}-\frac{1}{R e} \frac{\partial \overline{u_{i}^{\prime} u_{j}^{\prime}}}{\partial x_{j}}, \quad i=1,2, \\
\frac{\partial \bar{u}_{i}}{\partial x_{i}}=0
\end{gathered}
$$


Nas Eqs. (2.38) e (2.39), $\bar{u}_{i}$ é a i-ésima componente da velocidade média, $\bar{p}$ é a pressão média e $\overline{u_{i}^{\prime} u_{j}^{\prime}}$ é o tensor das tensões de Reynolds, este definido na seção seguinte.

\subsection{Modelagem $\kappa-\varepsilon$ da Turbulência}

Para a simulação dos efeitos da turbulência considerou-se a modelagem $\kappa-\varepsilon$, em que a ideia básica é acoplar às equações médias de Navier-Stokes (2.38)-(2.39) duas EDPs de transporte: uma para $\kappa$, a energia cinética da turbulência, e outra para $\varepsilon$, a dissipação da energia cinética $\kappa$. O objetivo é descrever a evolução da viscosidade adicional $\nu_{t}$ dada por (2.46). Em resumo, as variáveis turbulentas $\kappa$ e $\varepsilon$ são definidas, respectivamente, por

$$
\kappa=\frac{1}{2} \overline{u_{i}^{\prime} u_{i}^{\prime}} \quad \varepsilon=\nu\left(\frac{\overline{\partial u_{i}}}{\partial x_{j}}\right)^{2},
$$

em que $u_{i}^{\prime}$ é a componente flutuante da velocidade $u_{i}$.

Conhecidos $\kappa, \varepsilon$ e $\nu_{t}$, o tensor das tensões de Reynolds é definido por

$$
\overline{u_{i}^{\prime} u_{j}^{\prime}}=-\nu_{t} D_{i j}+\frac{2}{3} \kappa \delta_{i j}, \quad i=1,2,
$$

em que $\delta_{i j}$ representa o delta de Kronecker e $D_{i j}$, o tensor de deformações médio, dado por

$$
D_{i j}=\frac{\partial \bar{u}_{i}}{\partial x_{j}}+\frac{\partial \bar{u}_{j}}{\partial x_{i}}
$$

Em suma, as EDPs do modelo $\kappa-\varepsilon$ da turbulência são dadas por

$$
\begin{aligned}
\frac{\partial \kappa}{\partial t}+\frac{\partial\left(\kappa u_{j}\right)}{\partial x_{j}} & =\frac{1}{R_{e}} \frac{\partial}{\partial x_{j}}\left(\left(1+\frac{\nu_{t}}{\sigma_{\kappa}}\right) \frac{\partial \kappa}{\partial x_{j}}\right)+\nu_{t} D_{i j} \frac{\partial u_{i}}{\partial x_{j}}-\varepsilon \\
\frac{\partial \varepsilon}{\partial t}+\frac{\partial\left(\varepsilon u_{j}\right)}{\partial x_{j}} & =\frac{1}{R_{e}} \frac{\partial}{\partial x_{j}}\left(\left(1+\frac{\nu_{t}}{\sigma_{\varepsilon}}\right) \frac{\partial \varepsilon}{\partial x_{j}}\right)+\left(C_{1 \varepsilon} \nu_{t} D_{i j} \frac{\partial u_{i}}{\partial x_{j}}-C_{2 \varepsilon} \varepsilon\right) / T_{t}
\end{aligned}
$$

em que a escala de tempo do movimento turbulento e a viscosidade turbulenta são definidas, respectivamente, por

$$
\begin{gathered}
T_{t}=\kappa / \varepsilon, \\
\nu_{t}=C_{\mu} \kappa T_{t} .
\end{gathered}
$$

Nas equações acima apresentadas tem-se que $\sigma_{\kappa}=1.0$ e $\sigma_{\varepsilon}=1.3$ são coeficientes de difusão turbulentos, e $C_{1 \varepsilon}=1.44, C_{2 \varepsilon}=1.92$ e $C_{\mu}=0.09$ são constantes empíricas. Mais detalhes sobre essa modelagem da turbulência, pode-se recorrer a Ferreira [18].

As condições de contorno adotadas na simulação deste modelo de turbulência são as mesmas descritas na Seção 2.3 para o caso laminar. Já as condições iniciais aqui consideradas são 
definidas por Brandi [7], da forma

$$
\kappa=0.08 R e, \quad \varepsilon=\frac{100 \kappa}{3} \sqrt{\frac{\kappa}{R e}} .
$$

Para as variáveis turbulentas $\kappa \mathrm{e} \varepsilon$, as condições de contorno na entrada também são dadas por (2.47), enquanto que na saída são calculadas pela condição de Neumann:

$$
\frac{\partial \kappa}{\partial n}=0 \quad \frac{\partial \varepsilon}{\partial n}=0
$$

No contorno rígido são aplicadas as leis clássicas de parede descritas em [16, 52]. Finalmente, as condições de contorno na superfície livre são dadas por (ver [7, 18])

$$
\begin{array}{r}
p+\frac{2}{3 R e} \kappa-\frac{2}{R e}\left(1+\nu_{t}\right)\left[\frac{\partial u}{\partial x} n_{x}^{2}+\frac{\partial v}{\partial y} n_{y}^{2}+\left(\frac{\partial u}{\partial y}+\frac{\partial v}{\partial x}\right) n_{x} n_{y}\right]=0 \\
\frac{1}{R e}\left(1+\nu_{t}\right)\left[2 \frac{\partial u}{\partial x} m_{x} n_{x}+2 \frac{\partial v}{\partial y} m_{y} n_{y}+\left(\frac{\partial u}{\partial y}+\frac{\partial v}{\partial x}\right)\left(m_{x} n_{y}+m_{y} n_{x}\right)\right]=0 .
\end{array}
$$




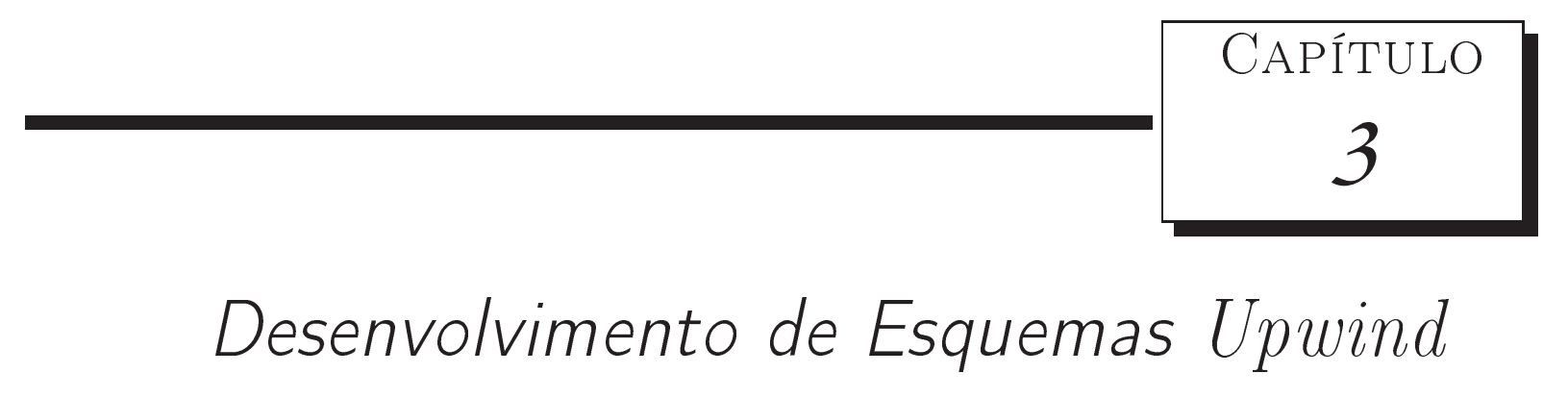

Neste capítulo apresenta-se um embasamento teórico para o desenvolvimento de esquemas convectivos upwind de alta resolução TVD, incluindo conceitos e restrições no que diz respeito à aproximação dos termos convectivos.

\subsection{Aproximação dos Termos Convectivos}

Nos últimos anos, esforço considerável tem sido feito para se obter soluções numéricas de boa qualidade para leis de conservação hiperbólicas e equações de Navier-Stokes, as quais são significativamente afetadas pela escolha do esquema de discretização para os termos convectivos (em geral não lineares). A principal dificuldade é controlar o fenômeno da difusão numérica.

A difusão numérica pode ser de duas formas (ver Figura 3.1 (a) e (b)): dissipativa e dispersiva. A Figura 3.1 (a) ilustra o efeito dissipativo (suavização de gradientes) de esquemas de primeira ordem (por exemplo, o esquema FOU [13]), e a Figura 3.1 (b) mostra o efeito dispersivo (surgimento de oscilações não físicas) de esquemas de alta ordem (por exemplo, o método de Lax-Wendroff [28]). De fato, esses inconvenientes estão contemplados no teorema de Godunov [48], o qual afirma que nenhum esquema de alta ordem linear pode ser monotônico. Neste contexto surge a motivação pela busca de esquemas convectivos upwind de alta ordem não lineares, como aquele apresentado na Figura 3.1 (c), os quais ajustam a ordem de precisão de acordo com a solução local de modo a manter comportamento limitado da solução.

Uma aproximação para os termos convectivos é obtida utilizando-se a estratégia upwind. Nessa estratégia, o termo convectivo é aproximado de acordo com o sinal da velocidade de convecção local. Para isso, consideram-se três nós computacionais adjacentes ao ponto de discretização, isto é, o a jusante D (Downstream), o a montante U (Upstream) e o mais a montante $\mathbf{R}$ (Remote-upstream). A Figura (3.2) ilustra esta estratégia, onde pode-se observar 
(a) Esquema de primeira ordem

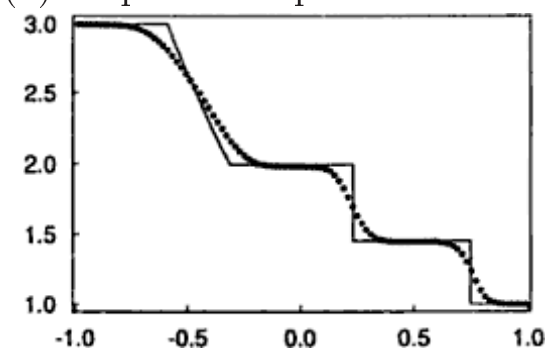

(b) Esquema de segunda ordem

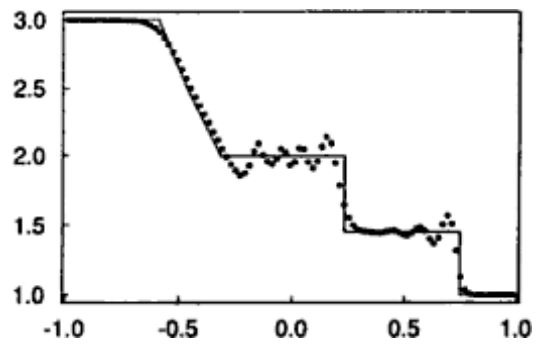

(c) Esquema de alta ordem

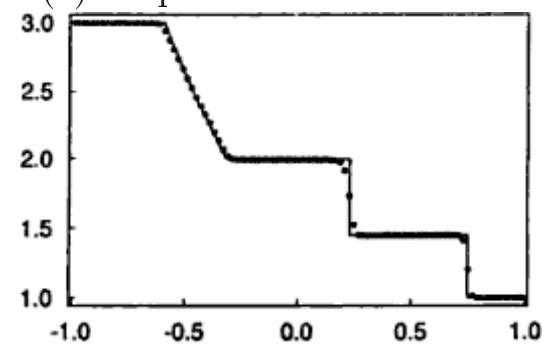

Figura 3.1: efeitos de difusão numérica: comparação entre o perfil característico de esquemas de primeira, segunda e alta ordem de precisão.

que as posições de $\mathbf{D}, \mathbf{U}$ e $\mathbf{R}$ são adotadas de acordo com o sinal da velocidade local $V_{f}$, na face $f$, de uma variável convectada $\phi_{f}$.
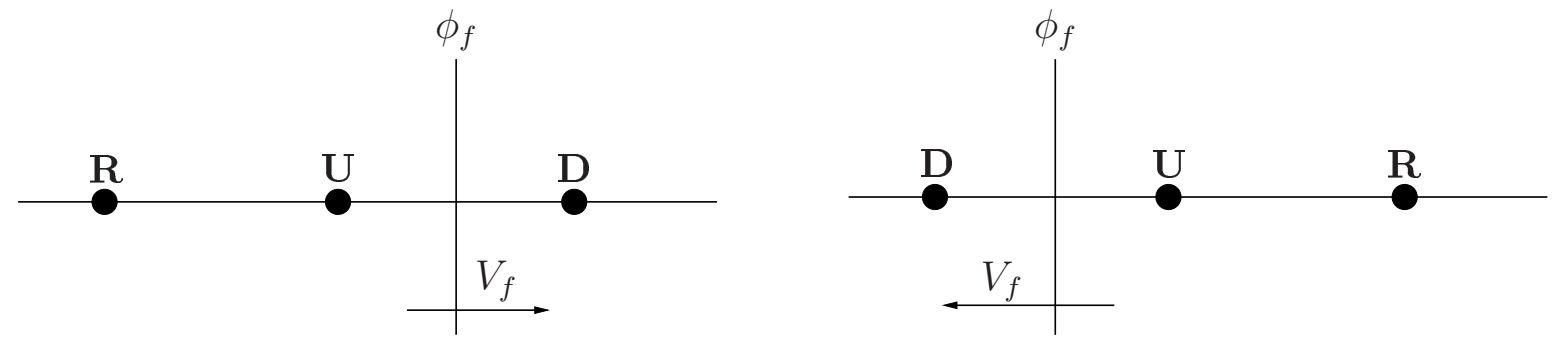

Figura 3.2: posição dos nós computacionais D, U e $\mathbf{R}$.

Nesse contexto, utilizando-se os três nós computacionais $\mathbf{D}, \mathbf{U}$ e $\mathbf{R}$, é possível escrever um esquema de convecção upwind dependente destes três pontos para avaliar $\phi_{f}$, através da seguinte relação:

$$
\phi_{f}=\phi_{f}\left(\phi_{D}, \phi_{U}, \phi_{R}\right)
$$

\subsection{Variáveis Normalizadas}

O conceito de variáveis normalizadas (NV - Normalized Variable) foi introduzido por Leonard [30] e é de grande importância para o desenvolvimento de esquemas de alta resolução, pois, juntamente com o critério TVD (ver Seção 3.5) introduzido por Harten [24], são capazes de obter 
esquemas estáveis e resolver gradientes elevados com alta precisão. Sendo $\phi$ a variável convectada, a variável normalizada de Leonard é definida por

$$
\hat{\phi}_{()}=\frac{\phi_{()}-\phi_{R}}{\phi_{D}-\phi_{R}}
$$

em que $\phi_{D}$ e $\phi_{R}$ são os valores não normalizados da propriedade $\phi$ nos pontos $\mathbf{D}$ e $\mathbf{R}$, respectivamente.

A partir desta definição observa-se que $\hat{\phi}_{R}=0$ e $\hat{\phi}_{D}=1$. Ainda, se $\hat{\phi}_{U}=0$ então $\phi_{U}=\phi_{R}$, e, se $\hat{\phi}_{U}=1$ então $\phi_{U}=\phi_{D}$. Assim, conclui-se que qualquer esquema de convecção upwind que utilize somente os valores de $\phi$ nos pontos $\mathbf{D}, \mathbf{U}$ e $\mathbf{R}$ pode ser representado na forma funcional

$$
\hat{\phi}_{f}=\hat{\phi}_{f}\left(\hat{\phi}_{U}\right)
$$

Leonard [30] também propôs o diagrama de variáveis normalizadas (NVD - Normalized Variable Diagram) baseado na definição (3.2) e na relação funcional (3.3) com o objetivo de representar uma relação entre as variáveis normalizadas $\hat{\phi}_{f}$ e $\hat{\phi}_{U}$, sendo isso de grande valia na análise de um esquema convectivo. Na Figura 3.3, por exemplo, os esquemas QUICK (Quadratic Upstream Interpolation for Convective Kinematics), diferença central (de segunda ordem) e FOU são ilustrados em variáveis normalizadas.

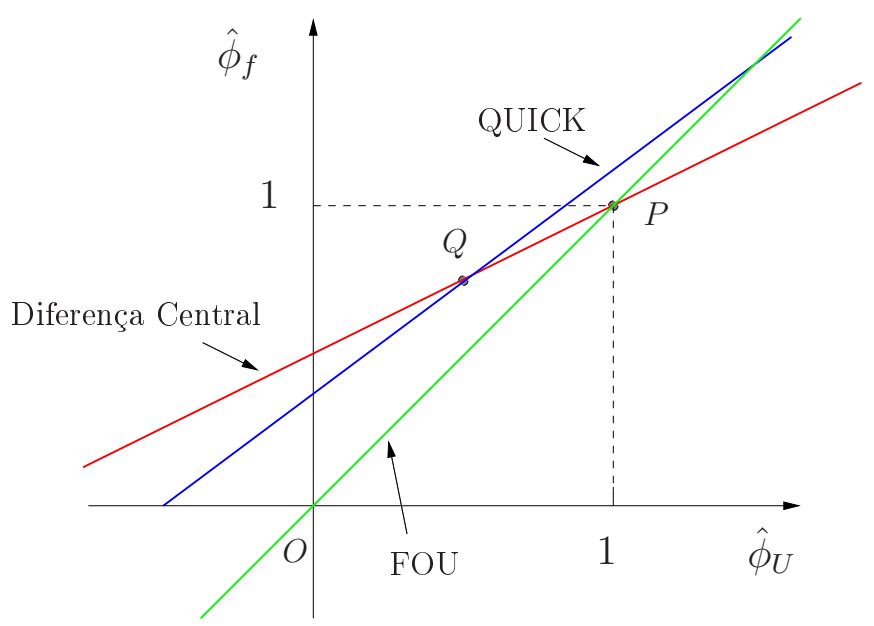

Figura 3.3: diagrama de variáveis normalizadas mostrando os esquemas QUICK, diferença central e FOU.

Utilizando-se deste diagrama, Leonard [30] mostrou que para se derivar um esquema monotônico de alta ordem não linear (ou linear por partes) formulado em NV, com $0 \leq \hat{\phi}_{U} \leq 1$, as seguintes condições devem ser satisfeitas: passar pelos pontos $O(0,0)$ e $P(1,1)$ (para ser monotônico), passar pelo ponto $Q(0.5,0.75)$ (para atingir segunda ordem de precisão) e passar pelo ponto $Q$ com inclinação 0.75 (para alcançar terceira ordem de precisão).

A explicação para o uso destas condições está no fato de que esquemas em variáveis nor- 
malizadas que têm interseção com o segundo quadrante podem produzir oscilações numéricas, assim como passar pelo quarto quadrante pode ser altamente difusivo (por isso o esquema deve passar pela origem $O$ ); e também, experimentos numéricos feitos por Leonard mostraram que os métodos em NVD que interceptam a reta $\hat{\phi}_{U}=1$ acima de $\hat{\phi}_{f}=1$ geram soluções oscilatórias, e os que interceptam a reta $\hat{\phi}_{U}=1$ abaixo de $\hat{\phi}_{f}=1$ geram dissipação numérica (por isso o esquema deve passar por $P$ ).

Leonard também recomenda que, para $\hat{\phi}_{U}<0$ e $\hat{\phi}_{U}>1$, seja utilizado o esquema FOU.

\subsection{Limitador de Fluxo}

Uma outra estratégia para derivar um esquema de alta resolução é o uso de limitadores de fluxo, os quais combinam um método de primeira ordem com um de ordem superior por meio de uma função não linear (ver, por exemplo, Waterson e Deconink [65]). O limitador de fluxo pode ser obtido reescrevendo-se a equação do esquema em variáveis não normalizadas na forma

$$
\phi_{f}=\phi_{U}+\frac{1}{2} \psi\left(r_{f}\right)\left(\phi_{D}-\phi_{U}\right)
$$

em que $\psi\left(r_{f}\right)=\psi_{f}$ é o limitador de fluxo e $r_{f}$ é a razão dos gradientes consecutivos (sensor), o qual em malhas uniformes é dado por

$$
r_{f}=\frac{\left(\frac{\partial \phi}{\partial x}\right)_{g}}{\left(\frac{\partial \phi}{\partial x}\right)_{f}} \approx \frac{\phi_{U}-\phi_{R}}{\phi_{D}-\phi_{U}} .
$$

Em variáveis normalizadas, as equações (3.4) e (3.5) são, respectivamente, da forma

$$
\begin{gathered}
\hat{\phi}_{f}=\hat{\phi}_{U}+\frac{1}{2} \psi_{f}\left(1-\hat{\phi}_{U}\right), \\
r_{f}=\frac{\hat{\phi}_{U}}{1-\hat{\phi}_{U}} .
\end{gathered}
$$

Nesse contexto, Sweby [54] introduziu o seu princípio de monotonicidade, dado por

$$
r_{f} \rightarrow 0 \longrightarrow \psi^{\prime}\left(r_{f}\right)=2
$$

Outra condição (necessária e suficiente), introduzida por Waterson e Deconinck [65], afirma que para o limitador atingir segunda ordem de precisão em malhas uniformes é

$$
\psi(1)=1
$$


Ainda, de acordo com Zijlema [70], para o limitador alcançar terceira ordem de precisão, uma condição necessária e suficiente é que ele satisfaça

$$
\psi^{\prime}(1)=\frac{1}{4} .
$$

\subsection{Critério de Limitação CBC}

No transporte de propriedades físicas, é de grande importância obter soluções numéricas limitadas, o que ocorre quando o valor da solução em um ponto computacional é limitado pelos valores da solução nos pontos vizinhos. A partir disso, Gaskell e Lau [21] formularam o critério de limitação CBC (Convection Boundedness Criterion), o qual afirma que um esquema definido por uma função contínua (ou contínua por partes) $\hat{\phi}_{f}=\hat{\phi}_{f}\left(\hat{\phi}_{U}\right)$ tem solução limitada se

$$
\begin{array}{rll}
\hat{\phi}_{U} \leq \hat{\phi}_{f}\left(\hat{\phi}_{U}\right) \leq 1, & \text { se } & \hat{\phi}_{U} \in[0,1], \\
\hat{\phi}_{f}\left(\hat{\phi}_{U}\right)=\hat{\phi}_{U}, & \text { se } & \hat{\phi}_{U} \notin[0,1], \\
\hat{\phi}_{f}(0)=0 & \text { e } & \hat{\phi}_{f}(1)=1 .
\end{array}
$$

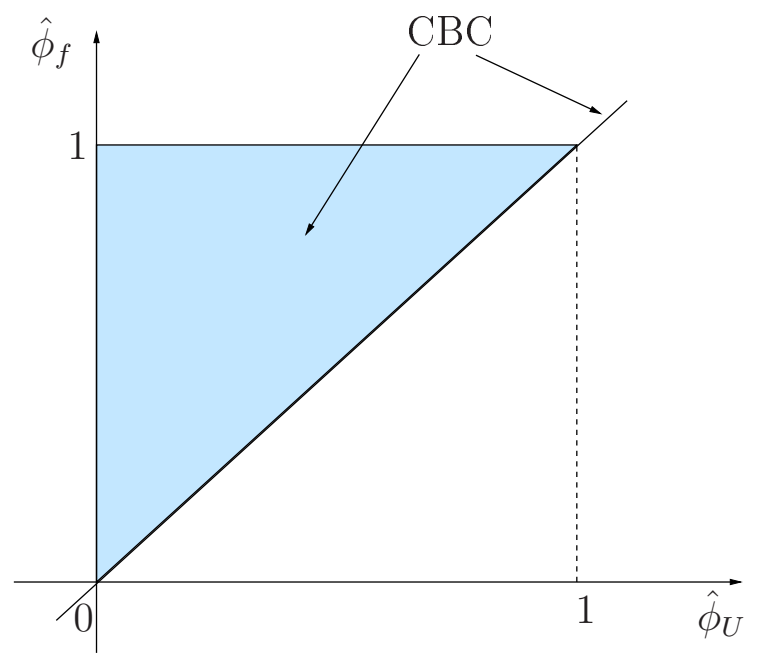

Figura 3.4: região CBC em variáveis normalizadas.

\subsection{Restrições TVD}

Outro requisito importante para um esquema de convecção é o conceito TVD (Total Variation Diminisching) proposto por Harten [24]. Esse conceito deu origem a restrições que, além da estabilidade da solução numérica, garantem sua convergência.

Considere uma sequência de aproximações discretas $\phi(t)=\phi_{i}(t)_{i \in \mathbf{Z}}$. A variação total (TV 
- Total Variation) no tempo $t$ desta sequência é definida por

$$
T V(\phi(t))=\sum_{i \in \mathbf{Z}}\left|\phi_{i+1}(t)-\phi_{i}(t)\right|
$$

E seja um esquema de diferença explícito envolvendo $(2 k+1)$ pontos dado por

$$
\phi_{i}^{n+1}=H\left(\phi_{i-k}^{n}, \ldots, \phi_{i+k}^{n}\right), \quad \forall n \geq 0, i \in \mathbf{Z},
$$

em que $H: \mathbf{R}^{2 k+1} \longrightarrow \mathbf{R}$ é uma função contínua e $\phi_{i}^{n}$ uma aproximação da solução exata $\phi$ nos pontos de malha $\left(x_{i}, t_{n}\right), \operatorname{com} x_{i}=i \delta x, t_{n}=n \delta t$, sendo $\delta x$ e $\delta t$ os espaçamentos (constantes) espacial e temporal, respectivamente.

Dentro desse contexto, o esquema (3.15) é dito TVD se, para todo conjunto de dados $\phi^{n}$, os valores $\phi^{n+1}$ calculados pelo método satisfizerem

$$
T V\left(\phi^{n+1}\right) \leq T V\left(\phi^{n}\right)
$$

É importante ressaltar que a variação total não precisa diminuir no sentido de decrescer, pois ela pode manter-se constante no tempo. O termo diminisching está se referindo ao fato de que a variação total não deve aumentar com o tempo.

De acordo com LeVeque [32], se um esquema é TVD, então os dados que são inicialmente monotônicos $\left(\phi_{i}^{n} \geq \phi_{i+1}^{n}\right.$, para todo $\left.i\right)$ permanecerão monotônicos em todos os passos de tempos futuros. Portanto, ao discretizar uma simples descontinuidade ela pode tornar-se suave durante o avanço temporal, porém sem um comportamento oscilatório. Essa propriedade leva a definir que um método é dito preservar monotonicidade se

$$
\phi_{i}^{n} \geq \phi_{i+1}^{n}, \forall i \quad \Rightarrow \quad \phi_{i}^{n+1} \geq \phi_{i+1}^{n+1}, \forall i
$$

Pode-se demonstrar que todo método TVD preserva monotonicidade.

Uma ferramenta fundamental para conseguir condições algébricas explícitas para um esquema ser TVD é o seguinte teorema, conhecido como critério de Harten:

Critério de Harten: considere um método geral da forma

$$
\phi_{i}^{n+1}=\phi_{i}^{n}+C_{i+1 / 2}\left(\phi_{i+1}^{n}-\phi_{i}^{n}\right)-D_{i-1 / 2}\left(\phi_{i}^{n}-\phi_{i-1}^{n}\right)
$$

em que os coeficientes $C_{i+1 / 2}$ e $D_{i-1 / 2}$ dependem das $2 k$ variáveis $\phi_{i-k+1}, \ldots, \phi_{i+k}$. Neste con- 
texto, se as seguintes condições são satisfeitas

$$
\begin{aligned}
C_{i+1 / 2} & \geq 0, \\
D_{i+1 / 2} & \geq 0, \\
C_{i+1 / 2}+D_{i+1 / 2} & \leq 1,
\end{aligned}
$$

então o esquema é TVD.

Vale ressaltar que esquemas TVD são muito atrativos do ponto de vista numérico, uma vez que garantem convergência e são monotônicos.

É possível visualizar o gráfico da região TVD para o limitador de fluxo usando o seguinte teorema (ver Sweby [54]):

Teorema 1 Se o limitador $\psi_{f}$ satisfaz as condições

$$
\begin{cases}0 \leq \psi\left(r_{f}\right) \leq \min \left(2 r_{f}, 2\right), & \text { se } \quad r_{f}>0 \\ \psi\left(r_{f}\right)=0, & \text { se } \quad r_{f} \leq 0\end{cases}
$$

então um esquema numérico é TVD sob a condição CFL (Courant-Friedrichs-Lewy) $\left|\theta=\frac{a \delta t}{\delta x}\right| \leq$ 1 , sendo a a velocidade de convecção.

A Figura 3.5 ilustra as condições (3.18) no plano $\psi_{f} \perp r_{f}$, em que o gráfico do limitador deve estar inteiramente contido na região hachurada.

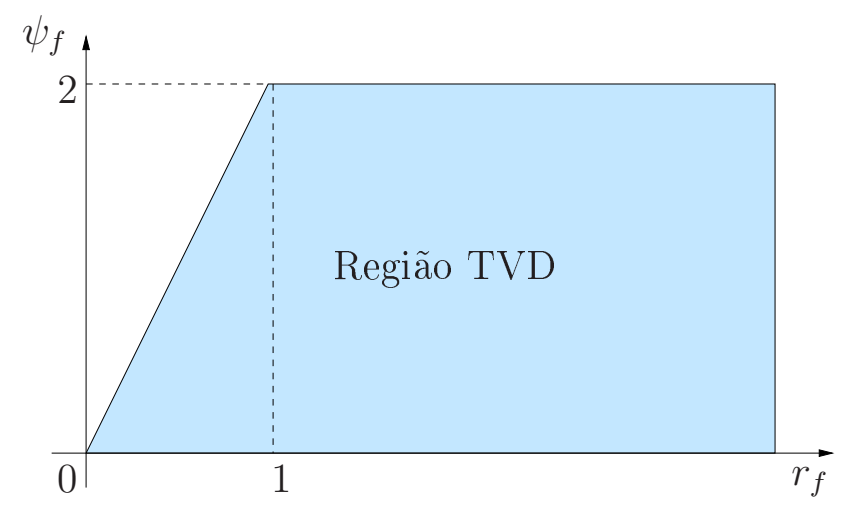

Figura 3.5: região TVD para o limitador de fluxo.

No contexto de variáveis normalizadas, a relação funcional entre $\hat{\phi}_{U}$ e $\hat{\phi}_{f}$ que satisfaz as restrições TVD pode ser expressa utilizando (3.18), (3.6) e (3.7), e é dada por

$$
\begin{cases}\hat{\phi}_{U} \leq \hat{\phi}_{f} \leq \min \left\{1,2 \hat{\phi}_{U}\right\}, & \text { se } 0<\hat{\phi}_{U}<1 \\ \hat{\phi}_{f}=\hat{\phi}_{U}, & \text { se } \hat{\phi}_{U} \leq 0 \text { ou } \hat{\phi}_{U} \geq 1\end{cases}
$$

Finalmente, para que um esquema convectivo $\hat{\phi}_{f}=\hat{\phi}_{f}\left(\hat{\phi}_{U}\right)$ seja TVD, o seu gráfico deve estar inteiramente contido na região hachurada na Figura 3.6. 


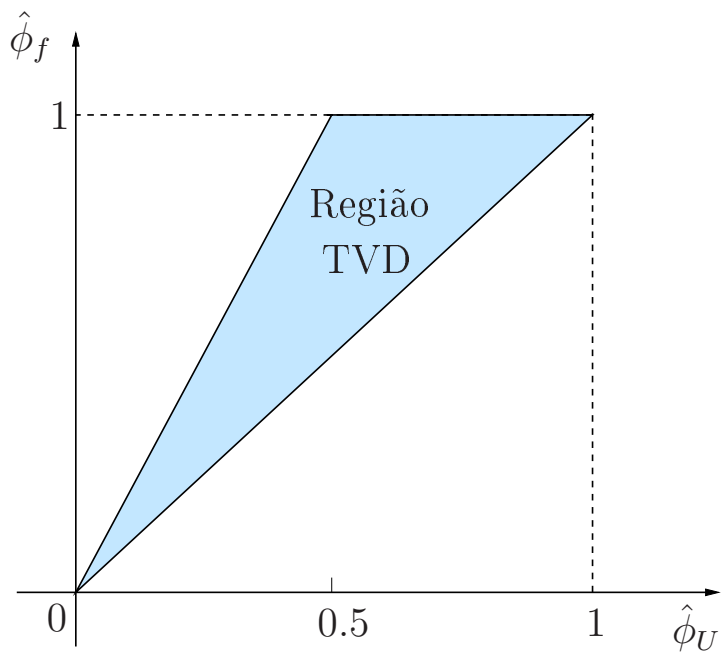

Figura 3.6: região TVD em variáveis normalizadas.

\subsection{Alguns Esquemas de Alta Resolução}

Com o objetivo de comparar o desempenho do esquema EPUS, nesta seção são apresentados alguns esquemas de alta resolução bem estabelecidos na literatura, a saber: WACEB [37], CUBISTA [1], Superbee [47], van Leer [61], MC [62], ADBQUICKEST [19], TOPUS [15] e SDPUS-C1 [35]. Neste trabalho, implementou-se estes esquemas nos contextos de diferenças e volumes finitos.

\subsubsection{WACEB}

Desenvolvido por Song et al. [37], o esquema WACEB (Weighted-Average Coefficient Ensuring Boundedness) é dado, em variáveis não normalizadas, por

$$
\phi_{f}= \begin{cases}\phi_{U}, & \text { se } \hat{\phi}_{U} \notin[0,1] \\ 2 \phi_{U}-\phi_{R}, & \text { se } 0 \leq \hat{\phi}_{U}<3 / 10, \\ \frac{1}{8}\left(3 \phi_{D}+6 \phi_{U}-\phi_{R}\right), & \text { se } 3 / 10 \leq \hat{\phi}_{U} \leq 5 / 6, \\ \phi_{D}, & \text { se } 5 / 6<\hat{\phi}_{U} \leq 1\end{cases}
$$

O limitador de fluxo deste esquema é dado por

$$
\psi\left(r_{f}\right)=\max \left\{0, \min \left[2 r_{f}, \frac{3+r_{f}}{4}, 2\right]\right\} .
$$

\subsubsection{CUBISTA}

O esquema CUBISTA (Convergent and Universally Bounded Interpolation Scheme for Treatment of Advection) foi desenvolvido por Alves et al. [1], sendo, em variáveis não normalizadas, 
dado por

$$
\phi_{f}= \begin{cases}\phi_{U}, & \text { se } \hat{\phi}_{U} \notin[0,1] \\ \frac{1}{4}\left(7 \phi_{U}-3 \phi_{R}\right), & \text { se } 0<\hat{\phi}_{U}<3 / 8, \\ \frac{1}{8}\left(3 \phi_{D}+6 \phi_{U}-\phi_{R}\right), & \text { se } 3 / 8 \leq \hat{\phi}_{U} \leq 3 / 4, \\ \frac{1}{4}\left(3 \phi_{D}+\phi_{U}\right), & \text { se } 3 / 4<\hat{\phi}_{U}<1 .\end{cases}
$$

O limitador de fluxo deste esquema é dado por

$$
\psi\left(r_{f}\right)=\max \left\{0, \min \left[2 r_{f}(-1+\theta), \frac{3+r_{f}}{4}, 2(1-\theta)\right]\right\}
$$

\subsubsection{Superbee}

Este método foi desenvolvido por Roe [47]. Na forma de variáveis não normalizadas é dado por

$$
\phi_{f}= \begin{cases}\phi_{U}, & \text { se } \hat{\phi}_{U} \notin[0,1] \\ 2 \phi_{U}-\phi_{R}, & \text { se } 0<\hat{\phi}_{U} \leq 1 / 3 \\ \frac{1}{2}\left(\phi_{D}+\phi_{U}\right), & \text { se } 1 / 3<\hat{\phi}_{U} \leq 1 / 2 \\ \frac{1}{2}\left(3 \phi_{U}-\phi_{R}\right), & \text { se } 1 / 2<\hat{\phi}_{U} \leq 2 / 3 \\ \phi_{D}, & \text { se } 2 / 3<\hat{\phi}_{U}<1\end{cases}
$$

O limitador de fluxo é dado por

$$
\psi\left(r_{f}\right)=\max \left\{0, \min \left(1,2 r_{f}\right), \min \left(2, r_{f}\right)\right\}
$$

\subsection{4 van Leer}

Desenvolvido originalmente por van Leer [61], este esquema em variáveis não normalizadas é da forma

$$
\phi_{f}=\left\{\begin{array}{lll}
\phi_{U}+(\varrho / 12)\left(\phi_{D}(3+\varrho)+\phi_{R}(\varrho-3)-2 \varrho \phi_{U}\right), & \text { se } & \hat{\phi}_{U} \in[0,1] \\
\phi_{U}, & \text { se } & \hat{\phi}_{U} \notin[0,1]
\end{array}\right.
$$

em que

$$
\varrho=\frac{2\left(1-\hat{\phi}_{U}\right) \hat{\phi}_{U}}{\left(1-\hat{\phi}_{U}\right)^{2}+\hat{\phi}_{U}}
$$

Seu limitador de fluxo pode ser escrito da forma

$$
\psi\left(r_{f}\right)=\frac{r_{f}+\left|r_{f}\right|}{1+\left|r_{f}\right|}
$$




\subsubsection{Monotonized Central-difference (MC)}

Este método foi proposto por van Leer em [62] e é dado, na forma de limitador de fluxo, por

$$
\psi\left(r_{f}\right)=\max \left\{0, \min \left(\frac{1+r_{f}}{2}, 2,2 r_{f}\right)\right\}
$$

\subsubsection{ADBQUICKEST}

O esquema ADaptative Bounded Quadratic Upstream for Convective Kinemactics with Estimated Streaming Terms, também denominado ADBQUICKEST, foi desenvolvido por Ferreira et al. [19]. Em variáveis não normalizadas é dado por

$$
\phi_{f}= \begin{cases}\phi_{U}, & \text { se } \hat{\phi}_{U} \notin[0,1], \\ (2-\theta) \phi_{U}-(1-\theta) \phi_{R}, & \text { se } 0<\hat{\phi}_{U}<a, \\ \left.\alpha_{D} \phi_{D}+\alpha_{U} \phi_{U}-\alpha_{R} \phi_{R}\right), & \text { se } a \leq \hat{\phi}_{U} \leq b, \\ (1-\theta) \phi_{D}+\theta \phi_{U}, & \text { se } b<\hat{\phi}_{U}<1\end{cases}
$$

em que

$$
\begin{aligned}
\alpha_{D} & =\frac{1}{6}\left(2-3|\theta|+\theta^{2}\right), \\
\alpha_{U} & =\frac{1}{6}\left(5+3|\theta|-2 \theta^{2}\right), \\
\alpha_{R} & =\frac{1}{6}\left(1-\theta^{2}\right) .
\end{aligned}
$$

Ainda, $a$ e $b$ são dados por

$$
a=\frac{2-|\theta|+\theta^{2}}{7-6 \theta-3|\theta|+2 \theta^{2}} \quad \text { e } \quad b=\frac{-4+6 \theta-3|\theta|+\theta^{2}}{-5+6 \theta-3|\theta|+2 \theta^{2}} .
$$

O limitador de fluxo deste esquema é dado por

$$
\psi\left(r_{f}\right)=\max \left\{0, \min \left[2 r_{f}, \frac{2+\theta^{2}-3 \theta+\left(1-\theta^{2}\right) r_{f}}{3-3 \theta}, 2\right]\right\}
$$

\subsubsection{TOPUS}

Desenvolvido por Queiroz [15], o esquema TOPUS (Third-Order Polynomial Upwind Scheme) é definido, em variáveis não normalizadas, por

$$
\phi_{f}=\left\{\begin{array}{lll}
\phi_{R}+\left(\phi_{D}-\phi_{R}\right)\left[\alpha \hat{\phi}_{U}^{4}+(-2 \alpha+1) \hat{\phi}_{U}^{3}+\left(\frac{5 \alpha-10}{4}\right) \hat{\phi}_{U}^{2}+\left(\frac{-\alpha+10}{4}\right) \hat{\phi}_{U}\right], & \text { se } \hat{\phi}_{U} \in[0,1] \\
\phi_{U}, & \text { se } \quad \hat{\phi}_{U} \notin[0,1]
\end{array}\right.
$$


O limitador de fluxo deste esquema é dado por

$$
\psi\left(r_{f}\right)=\max \left\{0, \frac{0.5\left(\left|r_{f}\right|+r_{f}\right)(-0.5 \alpha+1) r_{f}^{2}+(\alpha+4) r_{f}+(-0,5 \alpha+3)}{\left(1+\left|r_{f}\right|\right)^{3}}\right\} .
$$

Este esquema considera $\alpha \in[0,2]$, no entanto Queiroz [15] ressalta que os melhores resultados são obtidos para $\alpha=2$, uma vez que para esse valor o limitador de fluxo, neste caso, apresenta comportamento suave (ver, por exemplo, [65]). Em virtude disso, ao longo deste trabalho considerou-se $\alpha=2$.

\subsubsection{SDPUS-C1}

O esquema SDPUS-C1 (Six-Degree Polynomial Upwind Scheme - of $C^{1}$ class) foi introduzido por Lima [35]. Em variáveis não normalizadas é definido por

$$
\phi_{f}=\left\{\begin{array}{crc}
\phi_{R}+\left(\phi_{D}-\phi_{R}\right)\left[(-24+4 \gamma) \hat{\phi}_{U}^{6}+(68-12 \gamma) \hat{\phi}_{U}^{5}+\right. \\
\left.+(-64+13 \gamma) \hat{\phi}_{U}^{4}+(20-6 \gamma) \hat{\phi}_{U}^{3}+\gamma \hat{\phi}_{U}^{2}+\hat{\phi}_{U}\right], & \text { se } & \hat{\phi}_{U} \in[0,1], \\
\phi_{U}, & \text { se } & \hat{\phi}_{U} \notin[0,1] .
\end{array}\right.
$$

Seu limitador de fluxo é dado por

$$
\psi\left(r_{f}\right)=\max \left\{0, \frac{0.5\left(\left|r_{f}\right|+r_{f}\right)(-8+2 \gamma) r_{f}^{3}+(40-4 \gamma) r_{f}^{2}+2 \gamma r_{f}}{\left(1+\left|r_{f}\right|\right)^{5}}\right\}
$$

De acordo com Lima [35], o esquema SDPUS-C1 pode atingir até terceira ordem de precisão e, para $\gamma \in[4,13]$, o esquema está contido na região TVD, sendo que os melhores resultados foram obtidos para $\gamma=12$. Assim, ao longo deste trabalho utilizou-se $\gamma=12$.

\subsection{Estimativa de Erro}

Para uma melhor análise do desempenho dos esquemas numéricos, em alguns testes realizados calculou-se os erros relativos utilizando-se as normas $L_{1}, L_{2}$ e $L_{\infty}$, os quais são dados por

$$
\begin{aligned}
\left\|E_{h}\right\|_{1} & =\frac{\sum_{i=1}^{N}\left|u_{i, \text { exata }}-u_{i, \text { numérica }}\right|}{\sum_{i=1}^{N}\left|u_{i, \text { exata }}\right|}, \\
\left\|E_{h}\right\|_{2} & =\sqrt{\frac{\sum_{i=1}^{N}\left(u_{i, \text { exata }}-u_{i, \text { numérica }}\right)^{2}}{\sum_{i=1}^{N}\left(u_{i, \text { exata }}\right)^{2}}} \\
\left\|E_{h}\right\|_{\infty} & =\frac{\max _{1 \leq \mathrm{i} \leq \mathrm{N}}\left|\mathrm{u}_{\mathrm{i}, \text { exata }}-\mathrm{u}_{\mathrm{i}, \text { numérica }}\right|}{\max _{1 \leq \mathrm{i} \leq \mathrm{N}}\left|\mathrm{u}_{\mathrm{i}, \text { exata }}\right|}
\end{aligned}
$$


onde $N$ é o número de pontos da malha. Em alguns testes numéricos calculou-se também a ordem de convergência observada $\tilde{p}$ dos métodos numéricos. Considera-se uma estimativa assintótica para o erro relativo $\left\|E_{h}\right\|_{k}$, com $k=1,2, \infty$, dada da seguinte forma

$$
\left\|E_{h}\right\|_{k} \approx C h^{\tilde{p}}
$$

em que $C$ é uma constante dependente dos dados.

Ainda, pode-se escrever a partir de (3.39) que

$$
\left\|E_{\frac{h}{2}}\right\|_{k} \approx C\left(\frac{h}{2}\right)^{\tilde{p}}
$$

Dividindo (3.39) por (3.40), obtém-se

$$
\frac{\left\|E_{h}\right\|_{k}}{\left\|E_{\frac{h}{2}}\right\|_{k}} \approx \frac{h^{\tilde{p}}}{\left(\frac{h}{2}\right)^{\tilde{p}}}=2^{\tilde{p}} .
$$

Aplicando o logaritmo na Eq. (3.41), obtém-se a ordem de convergência observada por

$$
\tilde{p}=\frac{\left(\log \frac{\left\|E_{h}\right\|_{k}}{\left\|E_{\frac{h}{2}}\right\|_{k}}\right)}{\log 2} .
$$

Para mais detalhes sobre esta teoria, pode-se recorrer a [5]. 


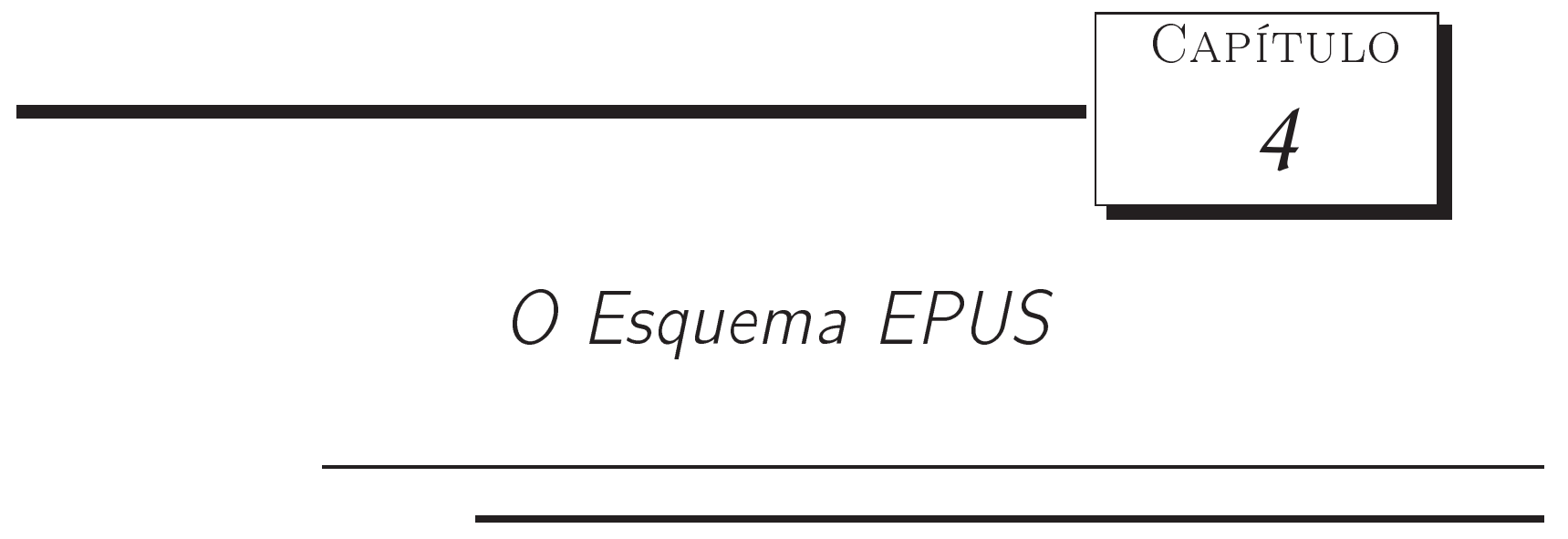

Neste capítulo apresenta-se o desenvolvimento do novo esquema upwind polinomial de alta resolução EPUS. Este esquema foi desenvolvido a partir de um polinômio de grau oito, e satisfaz as condições de Leonard e as restrições TVD/CBC, além de ser uma função de classe $C^{2}$ (propriedade esta adotada levando em consideração as recomendações de Lin e Chieng [36]).

\subsection{Derivação do Esquema EPUS}

Para o desenvolvimento do esquema EPUS, considerou-se um polinômio de grau oito no intervalo $[0,1]$ e fora deste intervalo o esquema FOU, isto é

$$
\hat{\phi}_{f}\left(\hat{\phi}_{U}\right)=\left\{\begin{array}{lll}
a_{8} \hat{\phi}_{U}^{8}+a_{7} \hat{\phi}_{U}^{7}+a_{6} \hat{\phi}_{U}^{6}+a_{5} \hat{\phi}_{U}^{5}+a_{4} \hat{\phi}_{U}^{4}+a_{3} \hat{\phi}_{U}^{3}+a_{2} \hat{\phi}_{U}^{2}+a_{1} \hat{\phi}_{U}+a_{0}, & \text { se } & \hat{\phi}_{U} \in[0,1], \\
\hat{\phi}_{U}, & \text { se } & \hat{\phi}_{U} \notin[0,1]
\end{array}\right.
$$

A determinação dos coeficientes $a_{0}, \ldots, a_{8}$ foi feita impondo-se, primeiramente, as condições de Leonard [30], como segue:

- passar pelo ponto $O(0,0)$;

- passar pelo ponto $P(1,1)$;

- passar pelo ponto $Q(0.5,0.75)$ (condição necessária e suficiente para atingir segunda ordem de precisão);

- ter inclinação de 0.75 no ponto $Q$ (condição necessária e suficiente para alcançar terceira ordem).

Aplicando tais condições em (4.1), tem-se 
$-\hat{\phi}_{f}(0)=0$ :

$$
a_{0}=0
$$

$-\hat{\phi}_{f}(1)=1$ :

$$
a_{8}+a_{7}+a_{6}+a_{5}+a_{4}+a_{3}+a_{2}+a_{1}=1 ;
$$

$-\hat{\phi}_{f}(0.5)=0.75$

$$
a_{8}+2 a_{7}+4 a_{6}+8 a_{5}+16 a_{4}+32 a_{3}+64 a_{2}+128 a_{1}=192 ;
$$

$-\hat{\phi}_{f}^{\prime}(0.5)=0.75$ :

$$
8 a_{8}+14 a_{7}+24 a_{6}+40 a_{5}+64 a_{4}+96 a_{3}+128 a_{2}+128 a_{1}=96 .
$$

Em adição a isso, para fechar o sistema, considerou-se que a função dada em (4.1) seja de classe $C^{2}$, isto é, deve possuir primeira e segunda derivadas contínuas nos pontos $(0,0)$ e $(1,1)$. De acordo com Lin e Chieng [36], impor que o esquema seja de classe $C^{1}$ evita problemas de convergência em malhas grosseiras. Esta ideia foi utilizada por Lima [35] no desenvolvimento de seu esquema polinomial SDPUS-C1 (ver Subseção 3.6.8). Neste trabalho, a continuidade da segunda derivada nos pontos $(0,0)$ e $(1,1)$ foi imposta na tentativa de se obter melhores resultados em problemas não lineares, visto que os exemplos numéricos confirmaram a nossa suspeita. Para tanto, as seguintes condições foram impostas:

$-\hat{\phi}_{f}^{\prime}(0)=1$ :

$$
a_{1}=1
$$

$-\hat{\phi}_{f}^{\prime}(1)=1$ :

$$
8 a_{8}+7 a_{7}+6 a_{6}+5 a_{5}+4 a_{4}+3 a_{3}+2 a_{2}+a_{1}=1 ;
$$

$-\hat{\phi}_{f}^{\prime \prime}(0)=0$ :

$$
a_{2}=0
$$

$-\hat{\phi}_{f}^{\prime \prime}(1)=0$ :

$$
56 a_{8}+42 a_{7}+30 a_{6}+20 a_{5}+12 a_{4}+6 a_{3}+2 a_{2}=0 .
$$

As duas primeiras condições impõem que o esquema seja de classe $C^{1}$ e as duas últimas condições 
de classe $C^{2}$.

Note que, impostas estas oito condições, tem-se um sistema formado por 8 equações e 9 incógnitas. Para o fechamento do sistema linear, considerou-se o coeficiente $a_{3}$ sendo um parâmetro livre, digamos $a_{3}=\lambda$. Com a resolução do sistema formado por (4.2)-(4.9) via WolframAlpha ${ }^{1}$, obtém-se

$$
\begin{gathered}
a_{8}=-4(\lambda-24) ; a_{7}=16(\lambda-23) ; a_{6}=(528-25 \lambda) ; a_{5}=(19 \lambda-336) ; \\
a_{4}=(80-7 \lambda) ; a_{3}=\lambda ; a_{2}=0 ; a_{1}=1 ; a_{0}=0 .
\end{gathered}
$$

Em resumo, o esquema EPUS em função do parâmetro livre $\lambda$ e em variáveis normalizadas é dado por

$$
\hat{\phi}_{f}=\left\{\begin{array}{rrr}
-4(\lambda-24) \hat{\phi}_{U}^{8}+16(\lambda-23) \hat{\phi}_{U}^{7}+(528-25 \lambda) \hat{\phi}_{U}^{6}+ & \\
+(19 \lambda-336) \hat{\phi}_{U}^{5}+(80-7 \lambda) \hat{\phi}_{U}^{4}+\lambda \hat{\phi}_{U}^{3}+\hat{\phi}_{U}, & \text { se } \hat{\phi}_{U} \in[0,1], \\
\hat{\phi}_{U}, & \text { se } \quad \hat{\phi}_{U} \notin[0,1] .
\end{array}\right.
$$

Utilizando a definição de variáveis normalizadas (NV) dada por (3.2), tem-se o esquema EPUS em variáveis não normalizadas:

$$
\phi_{f}=\left\{\begin{array}{rrr}
\phi_{R}+\left(\phi_{D}-\phi_{R}\right)\left[-4(\lambda-24) \hat{\phi}_{U}^{8}+16(\lambda-23) \hat{\phi}_{U}^{7}+(528-25 \lambda) \hat{\phi}_{U}^{6}+\right. & \\
\left.+(19 \lambda-336) \hat{\phi}_{U}^{5}+(80-7 \lambda) \hat{\phi}_{U}^{4}+\lambda \hat{\phi}_{U}^{3}+\hat{\phi}_{U}\right], & \text { se } & \hat{\phi}_{U} \in[0,1],(4.1 \\
\phi_{U}, & \text { se } & \hat{\phi}_{U} \notin[0,1] .
\end{array}\right.
$$

É importante observar que o novo esquema EPUS, apesar de ser um polinômio de grau oito no intervalo $[0,1]$, é de fácil implementação, pois é dado por apenas duas condicionais, ao passo que em outros esquemas, tais como WACEB [37], CUBISTA [1] ou WENO [4, 26], há muitas condicionais.

\subsection{Limitador de Fluxo do Esquema EPUS}

Derivado o esquema EPUS, pode-se determinar também o seu limitador de fluxo utilizando as seguintes equações:

$$
\begin{gathered}
\hat{\phi}_{f}=\hat{\phi}_{U}+\frac{1}{2} \psi\left(r_{f}\right)\left(1-\hat{\phi}_{U}\right), \\
\hat{\phi}_{U}=\frac{r_{f}}{1+r_{f}}
\end{gathered}
$$

\footnotetext{
${ }^{1}$ http://www.wolframalpha.com/
} 
onde $r_{f}$ é o sensor dado pela Eq. (3.7) e $\psi\left(r_{f}\right)$ é o limitador de fluxo. A expressão desse limitador é obtida na sequência.

Igualando (4.10) e (4.12), obtém-se, para a primeira sentença de (4.10)

$$
\begin{aligned}
&-4(\lambda-24) \hat{\phi}_{U}^{8}+16(\lambda-23) \hat{\phi}_{U}^{7}+(528-25 \lambda) \hat{\phi}_{U}^{6}+(19 \lambda-336) \hat{\phi}_{U}^{5}+ \\
&+(80-7 \lambda) \hat{\phi}_{U}^{4}+\lambda \hat{\phi}_{U}^{3}+\hat{\phi}_{U}=\hat{\phi}_{U}+\frac{1}{2} \psi\left(r_{f}\right)\left(1-\hat{\phi}_{U}\right) .
\end{aligned}
$$

A partir disso e utilizando a equação (4.13), tem-se

$$
\psi\left(r_{f}\right)=\frac{(2 \lambda-32) r_{f}^{5}+(160-4 \lambda) r_{f}^{4}+2 \lambda r_{f}^{3}}{\left(1+r_{f}\right)^{7}}
$$

Para a segunda sentença condicional do esquema em (4.10), tem-se que quando $\hat{\phi}_{f}=\hat{\phi}_{U}$, $\psi\left(r_{f}\right)=0$, o que é facilmente obtido por meio de (4.12).

Para se obter as condições na forma de limitador de fluxo para (4.10), note que quando $\psi\left(r_{f}\right)=0$, tem-se que $r_{f}<0$. De fato

$$
\begin{aligned}
& \hat{\phi}_{U} \notin[0,1] \Longleftrightarrow \hat{\phi}_{U}<0 \text { ou } \hat{\phi}_{U}>1, \\
& \hat{\phi}_{U}<0 \Longleftrightarrow \frac{r_{f}}{1+r_{f}}<0 \Longleftrightarrow r_{f}<0, \\
& \hat{\phi}_{U}>1 \Longleftrightarrow \frac{r_{f}}{1+r_{f}}>1 \Longleftrightarrow \text { absurdo. }
\end{aligned}
$$

Finalmente, o limitador de fluxo do esquema EPUS é dado por

$$
\psi\left(r_{f}\right)= \begin{cases}\frac{(2 \lambda-32) r_{f}^{5}+(160-4 \lambda) r_{f}^{4}+2 \lambda r_{f}^{3}}{\left(1+r_{f}\right)^{7}}, & \text { se } r_{f} \geq 0 \\ 0, & \text { se } r_{f}<0 .\end{cases}
$$

Em uma notação mais usual (ver, por exemplo, Waterson e Deconink [65]), o limitador de fluxo do esquema EPUS torna-se

$$
\psi\left(r_{f}\right)=\max \left\{0, \frac{0.5\left(\left|r_{f}\right|+r_{f}\right)\left[(2 \lambda-32) r_{f}^{4}+(160-4 \lambda) r_{f}^{3}+2 \lambda r_{f}^{2}\right]}{\left(1+\left|r_{f}\right|\right)^{7}}\right\} .
$$

\subsection{Considerações Relevantes sobre o Esquema EPUS}

A ordem formal do esquema EPUS é demonstrada como segue.

Utilizando a condição (3.9), verifica-se, para $r_{f} \geq 0$, que o esquema EPUS pode alcançar 
segunda ordem de precisão. De fato, pois

$$
\psi(1)=\frac{2 \lambda-32+160-4 \lambda+2 \lambda}{2^{7}}=1 .
$$

Ainda, utilizando a condição (3.10), conclui-se também que o esquema EPUS pode atingir até terceira ordem de precisão. De fato, a derivada do limitador de fluxo (4.15) para $r_{f} \geq 0$ é:

$\psi^{\prime}\left(r_{f}\right)=\frac{\left[5(2 \lambda-32) r_{f}^{4}+4(160-4 \lambda) r_{f}^{3}+6 \lambda r_{f}^{2}\right]\left(1+r_{f}\right)^{7}-7\left[(2 \lambda-32) r_{f}^{5}+(160-4 \lambda) r_{f}^{4}+2 \lambda r_{f}^{3}\right]\left(1+r_{f}\right)^{6}}{\left(1+r_{f}\right)^{14}}$.

Substituindo $r_{f}$ por 1 na derivada acima, tem-se

$$
\begin{aligned}
\psi^{\prime}(1) & =\frac{[5(2 \lambda-32)+4(160-4 \lambda)+6 \lambda] 2^{7}-7[(2 \lambda-32)+(160-4 \lambda)+2 \lambda] 2^{6}}{2^{14}} \\
& =\frac{1}{4} .
\end{aligned}
$$

Com isso, é possível concluir que o esquema EPUS pode alcançar até terceira ordem de precisão.

Em contrapartida, o limitador de fluxo do esquema EPUS não satisfaz o princípio de monotonicidade de Sweby, definido por (3.8), já que ao substituir $\psi^{\prime}(0)$ em (4.18), obtém-se $\psi^{\prime}(0)=0$, não satisfazendo, assim, $\psi^{\prime}(0)=2$. No entanto, essa propriedade não interfere na monotonicidade do esquema EPUS, pois este é TVD.

A propriedade TVD do esquema EPUS em função do parâmetro livre $\lambda$ é discutida como segue.

Considerando que neste projeto busca-se um novo esquema TVD, demonstrou-se para quais valores do parâmetro livre $\lambda$ o esquema EPUS satisfaz esta restrição para estabilidade. O intervalo obtido foi $\lambda \in[16,95]$ (para mais detalhes, ver demonstração Apêndice A). A Figura 4.1 mostra o esquema EPUS na região TVD para os limitantes inferior e superior deste intervalo, sendo a Figura 4.1 (a) no plano $\hat{\phi}_{U} \perp \hat{\phi}_{f}$ e a Figura 4.1 (b) no plano $r_{f} \perp \psi\left(r_{f}\right)$.

Ainda, buscou-se definir também a escolha do parâmetro livre de acordo com o problema em questão. A partir de diversos testes numéricos realizados ao longo do desenvolvimento do projeto, observou-se que o limitante inferior do intervalo de parâmetros, isto é $\lambda=16$, é o que conduz a um melhor desempenho do esquema EPUS em problemas com condições inicias suaves. Enquanto que para o limitante superior $\lambda=95$, melhores resultados são obtidos pelo esquema EPUS em problemas com descontinuidades, pontos extremos e altos gradientes.

Finaliza-se este capítulo comentando que o esquema EPUS em variáveis não normalizadas dado pela Eq. (4.11) foi implementado no ambiente de simulação Freeflow e nos programas desenvolvidos em linguagem $\mathrm{C}$ para resolver as equações de advecção e Burgers. O limitador de fluxo para o esquema EPUS dado pela Eq. (4.16) foi incorporado ao ambiente de simulação CLAWPACK. 
(a)

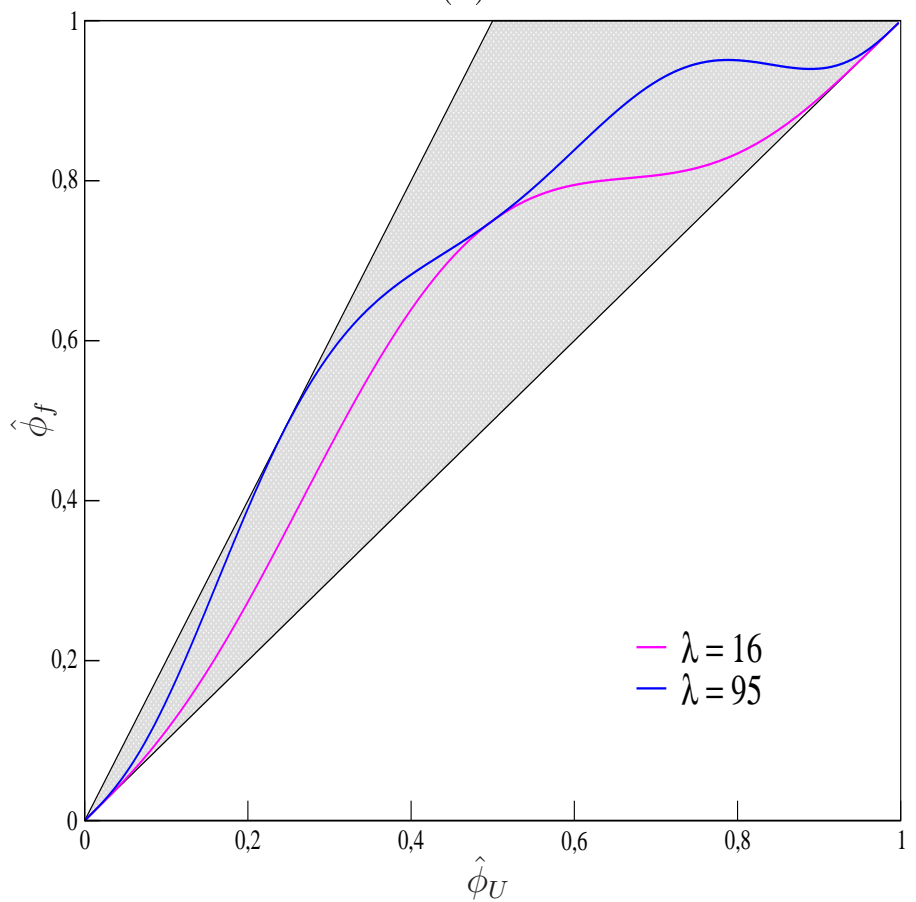

(b)

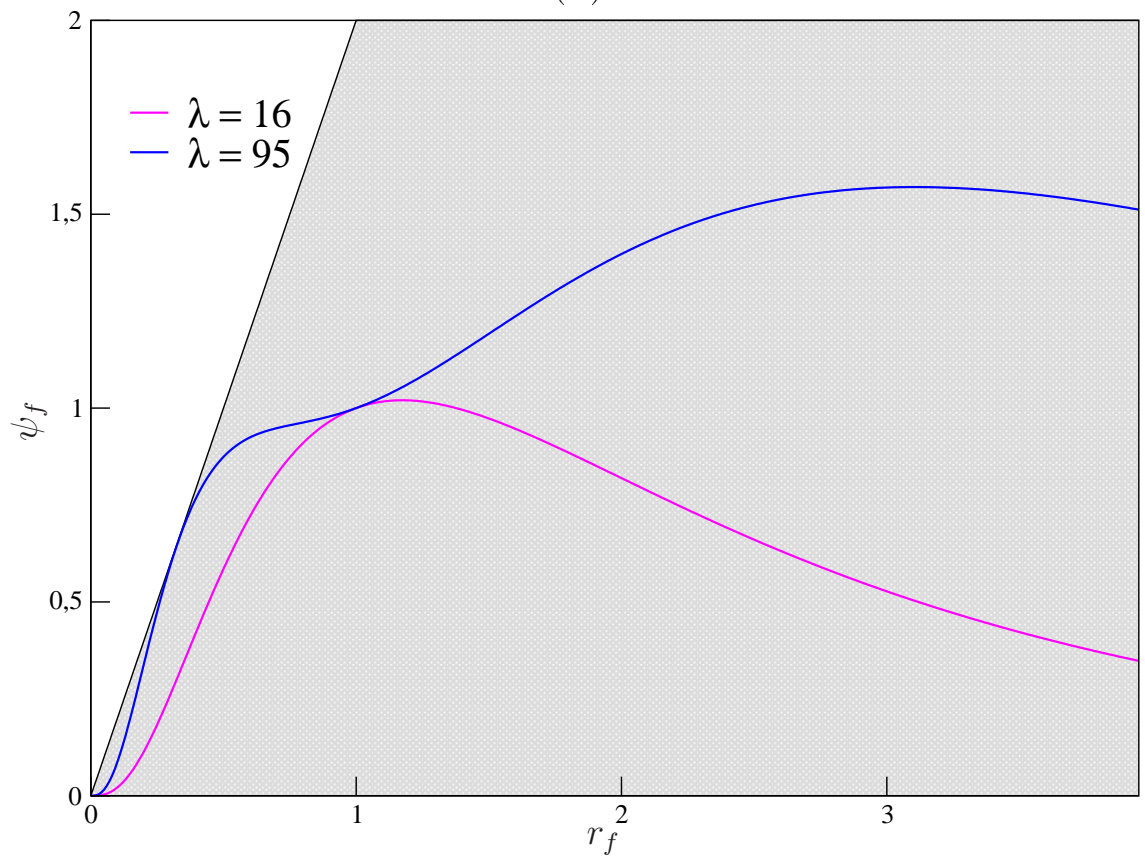

Figura 4.1: EPUS contido na região TVD para $\lambda=16$ e $\lambda=95$ (a) em variáveis normalizadas e (b) na forma de limitador de fluxo. 


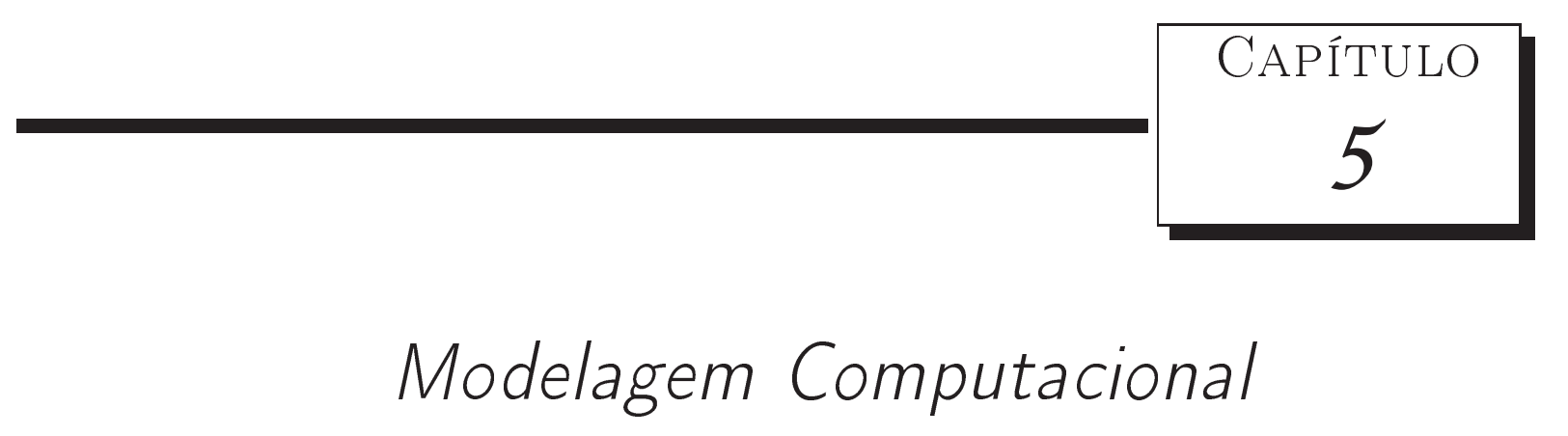

Neste capítulo apresenta-se a modelagem computacional das leis de conservação e das equações instantâneas e médias de Navier-Stokes, cuja formulação matemática é descrita no Capítulo 2. A discretização dessas equações é feita no contexto de diferenças finitas, em que as derivadas espaciais são aproximadas por diferença central de segunda ordem e a derivada temporal é aproximada por métodos de Runge-Kutta explícitos (Euler [20] e TVD de terceira ordem [56]).

\subsection{Discretização das Leis de Conservação 1D}

As leis de conservação 1D apresentadas na Eq. (2.1) são aproximadas, usando Euler explícito para a marcha no tempo, da seguinte forma

$$
\phi_{i}^{n+1}=\phi_{i}^{n}-\frac{\delta t}{\delta x}\left(F_{i+\frac{1}{2}}^{n}-F_{i-\frac{1}{2}}^{n}\right)
$$

em que $\phi_{i}^{n}$ representa a solução numérica da variável conservada $\phi$ no ponto $\left(x_{i}, t_{n}\right) \equiv\left(i \delta_{x}, n \delta_{t}\right) \equiv$ $(i, n)$. Os termos $F_{i+\frac{1}{2}}$ e $F_{i-\frac{1}{2}}$ denotam, respectivamente, os fluxos numéricos nas interfaces $f=i+\frac{1}{2}$ e $g=i-\frac{1}{2}$ das células computacionais, os quais são aqui aproximados pelos esquemas upwind de alta ordem de resolução.

No caso em que se usa o método Runge-Kutta TVD de terceira ordem para a discretização 
temporal, a aproximação numérica da EDP (2.1) é feita da forma

$$
\begin{aligned}
\phi^{(1)} & =\phi_{i}^{n}-\frac{\delta t}{\delta x}\left(F_{i+\frac{1}{2}}^{n}-F_{i-\frac{1}{2}}^{n}\right) \\
\phi^{(2)} & =\frac{3}{4} \phi_{i}^{n}+\frac{1}{4} \phi^{(1)}-\frac{1}{4} \frac{\delta t}{\delta x}\left(F_{i+\frac{1}{2}}^{(1)}-F_{i-\frac{1}{2}}^{(1)}\right) \\
\phi_{i}^{n+1} & =\frac{1}{3} \phi_{i}^{n}+\frac{2}{3} \phi^{(2)}-\frac{2}{3} \frac{\delta t}{\delta x}\left(F_{i+\frac{1}{2}}^{(2)}-F_{i-\frac{1}{2}}^{(2)}\right)
\end{aligned}
$$

em que $\phi^{(1)}$ e $\phi^{(2)}$ são variáveis auxiliares do método de Runge-Kutta. $F^{(1)}$ e $F^{(2)}$ nestas fórmulas representam os fluxos numéricos auxiliares calculados por algum esquema upwind de alta ordem de resolução. Doravante, o sobrescrito $n$ será omitido por simplicidade.

\subsubsection{Equação de Advecção}

Como visto na Seção 2.1.1, a função fluxo da equação de advecção é dada por $a u$, em que $a$ é uma constante (assumida igual a 1) que representa a velocidade advectiva. Considerando isso, a aproximação para a diferença dos fluxos numéricos $\left(F_{i+\frac{1}{2}}-F_{i-\frac{1}{2}}\right)$ em (5.1) e (5.2) é feita por

$$
F_{i+\frac{1}{2}}-F_{i-\frac{1}{2}}=\left.(a u)\right|_{i+\frac{1}{2}}-\left.(a u)\right|_{i-\frac{1}{2}}=a_{i+\frac{1}{2}} \cdot u_{i+\frac{1}{2}}-a_{i-\frac{1}{2}} \cdot u_{i-\frac{1}{2}}
$$

em que a variável convectada $u$ é aproximada pelos esquemas upwind de alta resolução nas faces $f$ e $g$. Nesta discretização considera-se o número de Courant $\theta=\frac{a \delta t}{\delta x}$, em que para a estabilidade deve-se satisfazer $\theta \leq 1$.

\subsubsection{Equação de Burgers}

Para esta equação tem-se um fluxo não linear convexo, o qual, como visto na Seção 2.1.2, é dado por $\frac{u^{2}}{2}$. A aproximação para a diferença dos fluxos numéricos $\left(F_{i+\frac{1}{2}}-F_{i-\frac{1}{2}}\right)$ em $(5.1) \mathrm{e}$ (5.2) é dada por

$$
F_{i+\frac{1}{2}}-F_{i-\frac{1}{2}}=\left.\left(\frac{u^{2}}{2}\right)\right|_{i+\frac{1}{2}}-\left.\left(\frac{u^{2}}{2}\right)\right|_{i-\frac{1}{2}}=\frac{1}{2}\left(\bar{u}_{i+\frac{1}{2}} \cdot u_{i+\frac{1}{2}}-\bar{u}_{i-\frac{1}{2}} \cdot u_{i-\frac{1}{2}}\right),
$$

em que as velocidades convectivas são dadas pelas médias

$$
\bar{u}_{i-\frac{1}{2}}=\frac{1}{2}\left(u_{i}+u_{i-1}\right) \quad \text { e } \quad \bar{u}_{i+\frac{1}{2}}=\frac{1}{2}\left(u_{i+1}+u_{i}\right) .
$$

A variável convectada $u$ nas faces $f$ e $g$ é calculada de maneira inteiramente análoga àquela da equação de adveç̧ão. Nesta discretização considera-se o número de Courant $\theta=\frac{\delta t}{\delta x} \leq 1$.

\subsubsection{Sistemas Hiperbólicos: Euler e Águas Rasas}

Estes dois sistemas hiperbólicos, os quais são descritos nas Seções 2.1.3, 2.2.1 e 2.2.2, são resolvidos numericamente neste trabalho utilizando o ambiente de simulação computacional 
CLAWPACK ${ }^{1}$ (Conservation LAW PACKage) de LeVeque [32]. Este ambiente foi desenvolvido no contexto de volumes finitos e contempla esquemas upwind de alta resolução na forma de limitadores de fluxo.

Em resumo, esse software utiliza, a critério do usuário, o método de Godunov de primeira ou de segunda ordem (sendo este último de primeira ordem com termo de correção, o qual foi proposto por LeVeque [32]). Para a utilização dos métodos de alta ordem de resolução, aplica-se neste termo de correção o limitador de fluxo desejado. No Apêndice C faz-se uma descrição sobre como o CLAWPACK resolve as leis de conservação aqui apresentadas. Para mais detalhes sobre esta resolução, ver LeVeque [32].

\subsection{Discretização das Leis de Conservação 2D}

Os sistemas hiperbólicos 2D de Euler e águas rasas, os quais foram descritos nas Seções 2.2.1 e 2.2.2, respectivamente, também foram resolvidos neste trabalho utilizando-se o software CLAWPACK. O método de resolução utilizado por este pacote computacional para o caso 2D é análogo ao caso 1D, por isso omite-se aqui sua descrição. Assim como a versão 1D do CLAWPACK, a versão 2D foi equipada com os esquemas de alta resolução aqui apresentados. Para mais detalhes, o leitor pode recorrer a LeVeque [32].

\subsection{Discretização das Equações de Navier-Stokes}

Nesta seção apresenta-se a discretização das equações de Navier-Stokes para os casos 2D, 3D e axissimétricos. Para a simulação destes escoamentos incompressíveis não estacionários utiliza-se o código Freeflow [10,41]. A metodologia numérica de cálculo utilizado neste código é a técnica GENSMAC (Generalized Simplified MAC) [58], sendo uma variante do método de projeção. Esta metodologia utiliza uma malha deslocada (ver descrição Seção 5.3.1) para a discretização do domínio de solução. Ainda, neste método, a marcha no tempo é feita usando-se o método de Euler explícito. Por fim, nos termos convectivos aplica-se para os fluxos numéricos os esquemas upwind de alta resolução apresentados na Seção 3.6.

\subsubsection{Malha Computacional}

Conforme visto, o método GENSMAC é implementado utilizando uma malha deslocada (ver Figura 5.1 para o caso 3D). Isso se deve ao fato de que, caso a malha não seja deslocada, a equação da quantidade de movimento na direção $x$, por exemplo, em um ponto $(i, j)$, é influenciada pela diferença de pressão $p_{i+1, j}-p_{i-1, j}$; o valor da pressão no próprio ponto $(i, j)$ não influencia diretamente essa equação, o que não satisfaz o problema físico, levando ao aparecimento de oscilações não físicas nas soluções (ver Fortuna [20]). Este tipo de malha foi apresentado por Harlow e Welch [23] e tem sido muito utlizado no cálculo de escoamentos incompressíveis. A malha deslocada, para o caso $3 D$, é composta por células com arestas $\delta x$,

\footnotetext{
${ }^{1}$ http://www.amath.washington.edu/ claw/
} 
$\delta y$ e $\delta z$. As três componentes da velocidade $u, v$ e $w$ são calculadas nas direções ilustradas na Figura 5.1.
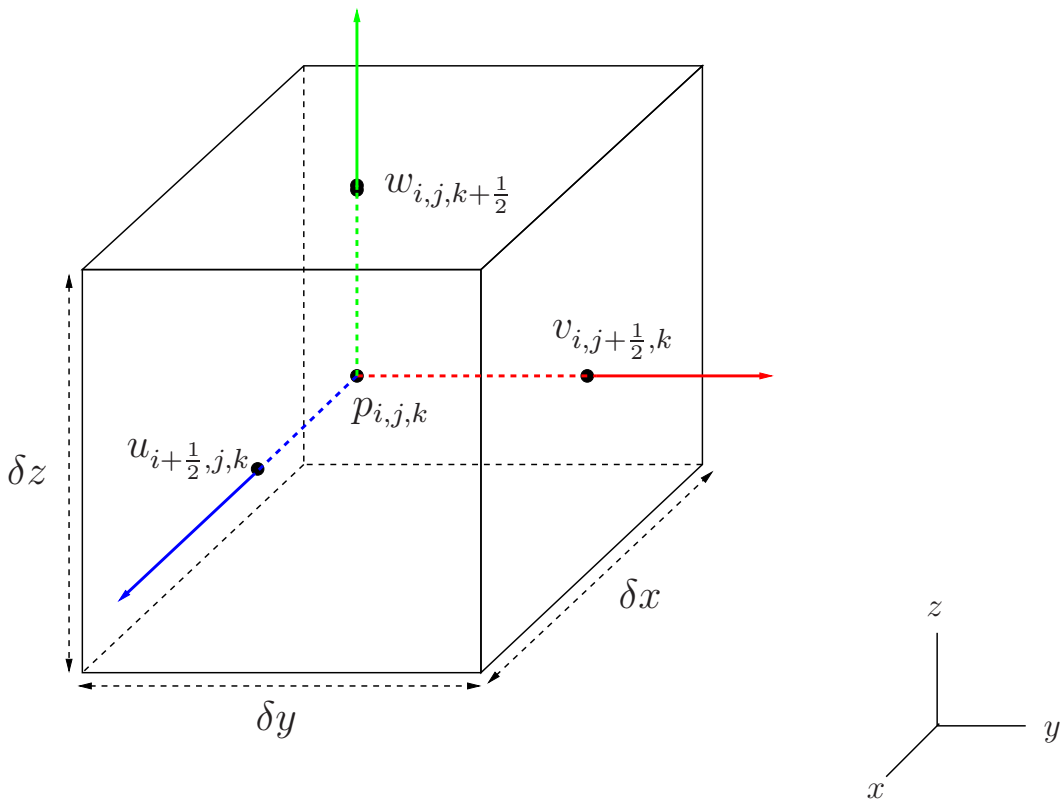

Figura 5.1: localização das componentes da velocidade numa típica célula computacional de uma malha deslocada 3D.

\subsubsection{Discretização dos Termos Convectivos - Caso Laminar}

As discretizações dos termos convectivos presentes nas equações instantâneas de Navier-Stokes são descritas neste trabalho apenas para o esquema EPUS, já que segue de maneira análoga para os demais esquemas de alta resolução. Considere, por exemplo, a propriedade convectada $\phi=u$ na equação de transporte de $u$, a qual é calculada na face $f$ utilizando a estratégia upwind conforme ilustrado na Figura 5.2. Na face $g$ desta figura os cálculos são semelhantes.

O termo convectivo em questão, isto é,

$$
\frac{\partial u_{j} u}{\partial x_{j}}=\left(\frac{\partial(u u)}{\partial x}+\frac{\partial(v u)}{\partial y}+\frac{\partial(w u)}{\partial z}\right)
$$

é avaliado na posição $\left(i+\frac{1}{2}, j, k\right)$ na Figura 5.1. Assim, a discretização é como segue:

$$
\begin{aligned}
\left.\left(\frac{\partial(u u)}{\partial x}+\frac{\partial(v u)}{\partial y}+\frac{\partial(w u)}{\partial z}\right)\right|_{i+\frac{1}{2}, j, k} \approx & \frac{\bar{u}_{1} u_{i+1, j, k}-\bar{u}_{2} u_{i, j, k}}{\delta x}+\frac{\bar{v}_{1} u_{i+\frac{1}{2}, j+\frac{1}{2}, k}-\bar{v}_{2} u_{i+\frac{1}{2}, j-\frac{1}{2}, k}}{\delta y} \\
& +\frac{\bar{w}_{1} u_{i+\frac{1}{2}, j, k+\frac{1}{2}}-\bar{w}_{2} u_{i+\frac{1}{2}, j, k-\frac{1}{2}}}{\delta z}
\end{aligned}
$$




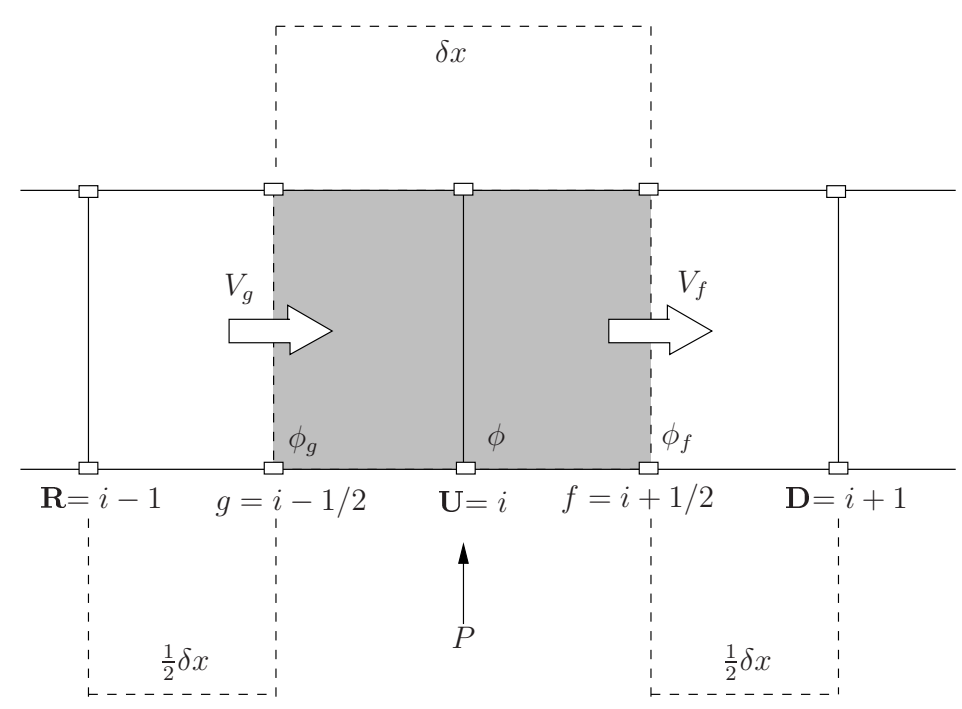

Figura 5.2: representação esquemática para aproximação dos termos convectivos, em que $P$ é o ponto de discretização e $\phi_{f}$ e $\phi_{g}$ são as velocidades de convecção nas faces $f$ e $g$, respectivamente.

em que os termos representados com uma barra são dados pelas médias, como por exemplo:

$$
\begin{aligned}
& \bar{v}_{1}=v_{i+\frac{1}{2}, j+\frac{1}{2}, k} \approx \frac{1}{2}\left(v_{i+1, j+\frac{1}{2}, k}+v_{i, j+\frac{1}{2}, k}\right), \\
& \bar{v}_{2}=v_{i+\frac{1}{2}, j-\frac{1}{2}, k} \approx \frac{1}{2}\left(v_{i+1, j-\frac{1}{2}, k}+v_{i, j-\frac{1}{2}, k}\right) .
\end{aligned}
$$

Na Eq. (5.9), os valores da propriedade convectada $u$ nos pontos $(i+1, j, k),(i, j, k)$, $\left(i+\frac{1}{2}, j+\frac{1}{2}, k\right),\left(i+\frac{1}{2}, j-\frac{1}{2}, k\right),\left(i+\frac{1}{2}, j, k+\frac{1}{2}\right)$ e $\left(i+\frac{1}{2}, j, k-\frac{1}{2}\right)$ são aproximados pelo novo esquema upwind de alta resolução EPUS.

Uma vez conhecidas as direções das velocidades advectivas $\bar{u}_{1}, \bar{u}_{2}, \bar{v}_{1}, \bar{v}_{2}, \bar{w}_{1}$ e $\bar{w}_{2}$ nas faces $(i+1, j, k),(i, j, k),\left(i+\frac{1}{2}, j+\frac{1}{2}, k\right),\left(i+\frac{1}{2}, j-\frac{1}{2}, k\right),\left(i+\frac{1}{2}, j, k+\frac{1}{2}\right)$ e $\left(i+\frac{1}{2}, j, k-\frac{1}{2}\right)$, respectivamente, os pontos vizinhos a jusante $\mathbf{D}$, a montante $\mathbf{U}$ e remoto a montante $\mathbf{R}$, em cada caso, ficam automaticamente especificados. Assim, usando-se a definição (3.2) de variáveis normalizadas de Leonard, seguem as aproximações para as velocidades convectadas nessas faces:

(1) Aproximação para $u_{i+1, j, k}$ :

- Se $\bar{u}_{1}=u_{i+1, j, k}>0$ e $\hat{u}_{U}=\frac{u_{i+\frac{1}{2}, j, k}-u_{i-\frac{1}{2}, j, k}}{u_{i+\frac{3}{2}, j, k}-u_{i-\frac{1}{2}, j, k}}$ então

$u_{i+1, j, k}= \begin{cases}u_{R}+\left(u_{D}-u_{R}\right)\left[-4(\lambda-24) \hat{u}_{U}^{8}+16(\lambda-23) \hat{u}_{U}^{7}+(528-25 \lambda) \hat{u}_{U}^{6}+\right. & \\ \left.+(19 \lambda-336) \hat{u}_{U}^{5}+(80-7 \lambda) \hat{u}_{U}^{4}+\lambda \hat{u}_{U}^{3}+\hat{u}_{U}\right], & \text { se } \hat{u}_{U} \in[0,1], \\ u_{U}, & \text { se } \quad \hat{u}_{U} \notin[0,1],\end{cases}$

em que 


$$
\mathbf{D}=\left(i+\frac{3}{2}, j, k\right), \quad \mathbf{U}=\left(i+\frac{1}{2}, j, k\right), \quad \mathbf{R}=\left(i-\frac{1}{2}, j, k\right)
$$

- Se $\bar{u}_{1}=u_{i+1, j, k}<0$ e $\hat{u}_{U}=\frac{u_{i+\frac{3}{2}, j, k}-u_{i+\frac{5}{2}, j, k}}{u_{i+\frac{1}{2}, j, k}-u_{i+\frac{5}{2}, j, k}}$ então

$u_{i+1, j, k}= \begin{cases}u_{R}+\left(u_{D}-u_{R}\right)\left[-4(\lambda-24) \hat{u}_{U}^{8}+16(\lambda-23) \hat{u}_{U}^{7}+(528-25 \lambda) \hat{u}_{U}^{6}+\right. & \\ \left.+(19 \lambda-336) \hat{u}_{U}^{5}+(80-7 \lambda) \hat{u}_{U}^{4}+\lambda \hat{u}_{U}^{3}+\hat{u}_{U}\right], & \text { se } \hat{u}_{U} \in[0,1], \\ u_{U}, & \text { se } \hat{u}_{U} \notin[0,1],\end{cases}$

em que

$$
\mathbf{D}=\left(i+\frac{1}{2}, j, k\right), \quad \mathbf{U}=\left(i+\frac{3}{2}, j, k\right), \quad \mathbf{R}=\left(i+\frac{5}{2}, j, k\right) ;
$$

(2) Aproximação para $u_{i, j, k}$ :

- Se $\bar{u}_{2}=u_{i, j, k}>0$ e $\hat{u}_{U}=\frac{u_{i-\frac{1}{2}, j, k}-u_{i-\frac{3}{2}, j, k}}{u_{i+\frac{1}{2}, j, k}-u_{i-\frac{3}{2}, j, k}}$ então

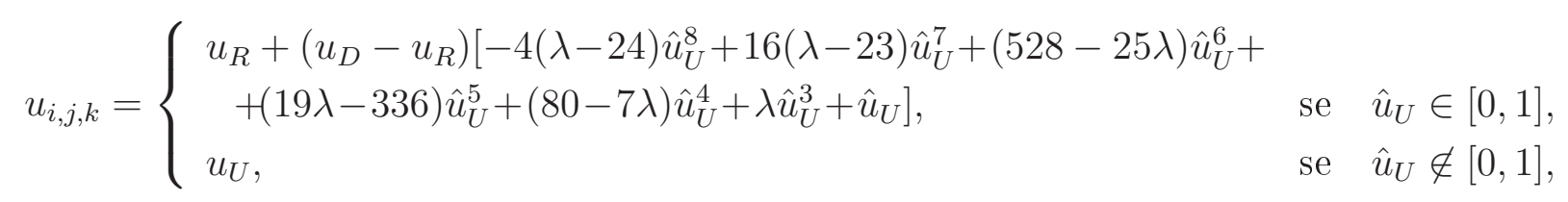

em que

$$
\begin{aligned}
& \mathbf{D}=\left(i+\frac{1}{2}, j, k\right), \quad \mathbf{U}=\left(i-\frac{1}{2}, j, k\right), \quad \mathbf{R}=\left(i-\frac{3}{2}, j, k\right) ; \\
& \text { - Se } \bar{u}_{2}=u_{i, j, k}<0 \text { e } \hat{u}_{U}=\frac{u_{i+\frac{1}{2}, j, k}-u_{i+\frac{3}{2}, j, k}}{u_{i-\frac{1}{2}, j, k}-u_{i+\frac{3}{2}, j, k}} \text { então } \\
& u_{i, j, k}=\left\{\begin{array}{lrl}
u_{R}+\left(u_{D}-u_{R}\right)\left[-4(\lambda-24) \hat{u}_{U}^{8}+16(\lambda-23) \hat{u}_{U}^{7}+(528-25 \lambda) \hat{u}_{U}^{6}+\right. & \\
\left.+(19 \lambda-336) \hat{u}_{U}^{5}+(80-7 \lambda) \hat{u}_{U}^{4}+\lambda \hat{u}_{U}^{3}+\hat{u}_{U}\right], & \text { se } \hat{u}_{U} \in[0,1], \\
u_{U}, & \text { se } \quad \hat{u}_{U} \notin[0,1],
\end{array}\right.
\end{aligned}
$$

em que

$$
\mathbf{D}=\left(i-\frac{1}{2}, j, k\right), \quad \mathbf{U}=\left(i+\frac{1}{2}, j, k\right), \quad \mathbf{R}=\left(i+\frac{3}{2}, j, k\right) ;
$$

(3) Aproximação para $u_{i+\frac{1}{2}, j+\frac{1}{2}, k}$ :

- Se $\bar{v}_{1}=v_{i+\frac{1}{2}, j+\frac{1}{2}, k}>0$ e $\hat{u}_{U}=\frac{u_{i+\frac{1}{2}, j, k}-u_{i+\frac{1}{2}, j-1, k}}{u_{i+\frac{1}{2}, j+1, k}-u_{i+\frac{1}{2}, j-1, k}}$ então

$u_{i+\frac{1}{2}, j+\frac{1}{2}, k}= \begin{cases}u_{R}+\left(u_{D}-u_{R}\right)\left[-4(\lambda-24) \hat{u}_{U}^{8}+16(\lambda-23) \hat{u}_{U}^{7}+(528-25 \lambda) \hat{u}_{U}^{6}+\right. & \\ \left.+(19 \lambda-336) \hat{u}_{U}^{5}+(80-7 \lambda) \hat{u}_{U}^{4}+\lambda \hat{u}_{U}^{3}+\hat{u}_{U}\right], & \text { se } \hat{u}_{U} \in[0,1], \\ u_{U}, & \text { se } \hat{u}_{U} \notin[0,1],\end{cases}$

em que 


$$
\mathbf{D}=\left(i+\frac{1}{2}, j+1, k\right), \quad \mathbf{U}=\left(i+\frac{1}{2}, j, k\right), \quad \mathbf{R}=\left(i+\frac{1}{2}, j-1, k\right)
$$

- Se $\bar{v}_{1}=v_{i+\frac{1}{2}, j+\frac{1}{2}, k}<0$ e $\hat{u}_{U}=\frac{u_{i+\frac{1}{2}, j+1, k}-u_{i+\frac{1}{2}, j+2, k}}{u_{i+\frac{1}{2}, j, k}-u_{i+\frac{1}{2}, j+2, k}}$ então

$u_{i+\frac{1}{2}, j+\frac{1}{2}, k}= \begin{cases}u_{R}+\left(u_{D}-u_{R}\right)\left[-4(\lambda-24) \hat{u}_{U}^{8}+16(\lambda-23) \hat{u}_{U}^{7}+(528-25 \lambda) \hat{u}_{U}^{6}+\right. & \\ \left.+(19 \lambda-336) \hat{u}_{U}^{5}+(80-7 \lambda) \hat{u}_{U}^{4}+\lambda \hat{u}_{U}^{3}+\hat{u}_{U}\right], & \text { se } \hat{u}_{U} \in[0,1], \\ u_{U}, & \text { se } \quad \hat{u}_{U} \notin[0,1],\end{cases}$

em que

$$
\mathbf{D}=\left(i+\frac{1}{2}, j, k\right), \quad \mathbf{U}=\left(i+\frac{1}{2}, j+1, k\right), \quad \mathbf{R}=\left(i+\frac{1}{2}, j+2, k\right) ;
$$

(4) Aproximação para $u_{i+\frac{1}{2}, j-\frac{1}{2}, k}$ :

- Se $\bar{v}_{2}=v_{i+\frac{1}{2}, j-\frac{1}{2}, k}>0$ e $\hat{u}_{U}=\frac{u_{i+\frac{1}{2}, j-1, k}-u_{i+\frac{1}{2}, j-2, k}}{u_{i+\frac{1}{2}, j, k}-u_{i+\frac{1}{2}, j-2, k}}$ então

$u_{i+\frac{1}{2}, j-\frac{1}{2}, k}= \begin{cases}u_{R}+\left(u_{D}-u_{R}\right)\left[-4(\lambda-24) \hat{u}_{U}^{8}+16(\lambda-23) \hat{u}_{U}^{7}+(528-25 \lambda) \hat{u}_{U}^{6}+\right. & \\ \left.+(19 \lambda-336) \hat{u}_{U}^{5}+(80-7 \lambda) \hat{u}_{U}^{4}+\lambda \hat{u}_{U}^{3}+\hat{u}_{U}\right], & \text { se } \hat{u}_{U} \in[0,1], \\ u_{U}, & \text { se } \hat{u}_{U} \notin[0,1],\end{cases}$

em que

$$
\mathbf{D}=\left(i+\frac{1}{2}, j, k\right), \quad \mathbf{U}=\left(i+\frac{1}{2}, j-1, k\right), \quad \mathbf{R}=\left(i+\frac{1}{2}, j-2, k\right)
$$

- Se $\bar{v}_{2}=v_{i+\frac{1}{2}, j-\frac{1}{2}, k}<0$ e $\hat{u}_{U}=\frac{u_{i+\frac{1}{2}, j, k}-u_{i+\frac{1}{2}, j+1, k}}{u_{i+\frac{1}{2}, j-1, k}-u_{i+\frac{1}{2}, j+1, k}}$ então

$u_{i+\frac{1}{2}, j-\frac{1}{2}, k}= \begin{cases}u_{R}+\left(u_{D}-u_{R}\right)\left[-4(\lambda-24) \hat{u}_{U}^{8}+16(\lambda-23) \hat{u}_{U}^{7}+(528-25 \lambda) \hat{u}_{U}^{6}+\right. & \\ \left.+(19 \lambda-336) \hat{u}_{U}^{5}+(80-7 \lambda) \hat{u}_{U}^{4}+\lambda \hat{u}_{U}^{3}+\hat{u}_{U}\right], & \text { se } \hat{u}_{U} \in[0,1], \\ u_{U}, & \text { se } \quad \hat{u}_{U} \notin[0,1],\end{cases}$

em que

$$
\mathbf{D}=\left(i+\frac{1}{2}, j-1, k\right), \quad \mathbf{U}=\left(i+\frac{1}{2}, j, k\right), \quad \mathbf{R}=\left(i+\frac{1}{2}, j+1, k\right) ;
$$

(5) Aproximação para $u_{i+\frac{1}{2}, j, k+\frac{1}{2}}$ :

- Se $\bar{w}_{1}=w_{i+\frac{1}{2}, j, k+\frac{1}{2}}>0$ e $\hat{u}_{U}=\frac{u_{i+\frac{1}{2}, j, k}-u_{i+\frac{1}{2}, j, k-1}}{u_{i+\frac{1}{2}, j, k+1}-u_{i+\frac{1}{2}, j, k-1}}$ então

$u_{i+\frac{1}{2}, j, k+\frac{1}{2}}= \begin{cases}u_{R}+\left(u_{D}-u_{R}\right)\left[-4(\lambda-24) \hat{u}_{U}^{8}+16(\lambda-23) \hat{u}_{U}^{7}+(528-25 \lambda) \hat{u}_{U}^{6}+\right. & \\ \left.+(19 \lambda-336) \hat{u}_{U}^{5}+(80-7 \lambda) \hat{u}_{U}^{4}+\lambda \hat{u}_{U}^{3}+\hat{u}_{U}\right], & \text { se } \hat{u}_{U} \in[0,1], \\ u_{U}, & \text { se } \quad \hat{u}_{U} \notin[0,1]\end{cases}$ em que 


$$
\mathbf{D}=\left(i+\frac{1}{2}, j, k+1\right), \quad \mathbf{U}=\left(i+\frac{1}{2}, j, k\right), \quad \mathbf{R}=\left(i+\frac{1}{2}, j, k-1\right)
$$

- Se $\bar{w}_{1}=w_{i+\frac{1}{2}, j, k+\frac{1}{2}}<0$ e $\hat{u}_{U}=\frac{u_{i+\frac{1}{2}, j, k+1}-u_{i+\frac{1}{2}, j, k+2}}{u_{i+\frac{1}{2}, j, k}-u_{i+\frac{1}{2}, j, k+2}}$ então

$u_{i+\frac{1}{2}, j, k+\frac{1}{2}}= \begin{cases}u_{R}+\left(u_{D}-u_{R}\right)\left[-4(\lambda-24) \hat{u}_{U}^{8}+16(\lambda-23) \hat{u}_{U}^{7}+(528-25 \lambda) \hat{u}_{U}^{6}+\right. & \\ \left.+(19 \lambda-336) \hat{u}_{U}^{5}+(80-7 \lambda) \hat{u}_{U}^{4}+\lambda \hat{u}_{U}^{3}+\hat{u}_{U}\right], & \text { se } \hat{u}_{U} \in[0,1], \\ u_{U}, & \text { se } \hat{u}_{U} \notin[0,1],\end{cases}$

em que

$$
\mathbf{D}=\left(i+\frac{1}{2}, j, k\right), \quad \mathbf{U}=\left(i+\frac{1}{2}, j, k+1\right), \quad \mathbf{R}=\left(i+\frac{1}{2}, j, k+2\right)
$$

(6) Aproximação para $u_{i+\frac{1}{2}, j, k-\frac{1}{2}}$ :

- Se $\bar{w}_{2}=w_{i+\frac{1}{2}, j, k-\frac{1}{2}}>0$ e $\hat{u}_{U}=\frac{u_{i+\frac{1}{2}, j, k-1}-u_{i+\frac{1}{2}, j, k-2}}{u_{i+\frac{1}{2}, j, k}-u_{i+\frac{1}{2}, j, k-2}}$ então

$u_{i+\frac{1}{2}, j, k-\frac{1}{2}}= \begin{cases}u_{R}+\left(u_{D}-u_{R}\right)\left[-4(\lambda-24) \hat{u}_{U}^{8}+16(\lambda-23) \hat{u}_{U}^{7}+(528-25 \lambda) \hat{u}_{U}^{6}+\right. & \\ \left.+(19 \lambda-336) \hat{u}_{U}^{5}+(80-7 \lambda) \hat{u}_{U}^{4}+\lambda \hat{u}_{U}^{3}+\hat{u}_{U}\right], & \text { se } \hat{u}_{U} \in[0,1], \\ u_{U}, & \text { se } \quad \hat{u}_{U} \notin[0,1],\end{cases}$

em que

$$
\mathbf{D}=\left(i+\frac{1}{2}, j, k\right), \quad \mathbf{U}=\left(i+\frac{1}{2}, j, k-1\right), \quad \mathbf{R}=\left(i+\frac{1}{2}, j, k-2\right) ;
$$

- Se $\bar{w}_{2}=w_{i+\frac{1}{2}, j, k-\frac{1}{2}}<0$ e $\hat{u}_{U}=\frac{u_{i+\frac{1}{2}, j, k}-u_{i+\frac{1}{2}, j, k+1}}{u_{i+\frac{1}{2}, j, k-1}-u_{i+\frac{1}{2}, j, k+1}}$ então

$u_{i+\frac{1}{2}, j, k-\frac{1}{2}}= \begin{cases}u_{R}+\left(u_{D}-u_{R}\right)\left[-4(\lambda-24) \hat{u}_{U}^{8}+16(\lambda-23) \hat{u}_{U}^{7}+(528-25 \lambda) \hat{u}_{U}^{6}+\right. & \\ \left.+(19 \lambda-336) \hat{u}_{U}^{5}+(80-7 \lambda) \hat{u}_{U}^{4}+\lambda \hat{u}_{U}^{3}+\hat{u}_{U}\right], & \text { se } \hat{u}_{U} \in[0,1], \\ u_{U}, & \text { se } \hat{u}_{U} \notin[0,1],\end{cases}$

em que

$$
\mathbf{D}=\left(i+\frac{1}{2}, j, k-1\right), \quad \mathbf{U}=\left(i+\frac{1}{2}, j, k\right), \quad \mathbf{R}=\left(i+\frac{1}{2}, j, k+1\right)
$$

A aproximação dos demais termos convectivos das equações instantâneas de Navier-Stokes segue de maneira análoga, assim como as aproximações por meio dos demais esquemas upwind de alta resolução citados no Capítulo 3.

\subsubsection{Discretização dos Termos Convectivos - Caso Turbulento}

As discretizações dos termos convectivos para as equações médias de Navier-Stokes com modelagem $\kappa-\varepsilon$ (caso turbulento) são feitas também apenas pelo esquema EPUS, já que segue de maneira análoga para os demais esquemas de alta resolução. 
Por simplicidade e sem perda de generalidade, considera-se apenas a propriedade transportada $\kappa$ da Eq. (2.43). O termo convectivo correspondente é

$$
\frac{\partial u_{j} \kappa}{\partial x_{j}}=\left(\frac{\partial(u \kappa)}{\partial x}+\frac{\partial(v \kappa)}{\partial y}\right)
$$

que é avaliado na posição $(i, j)$. Assim,

$$
\left.\left(\frac{\partial(u \kappa)}{\partial x}+\frac{\partial(v \kappa)}{\partial y}+\right)\right|_{i, j} \approx \frac{\bar{u}_{1} \kappa_{i+\frac{1}{2}, j}-\bar{u}_{2} \kappa_{i-\frac{1}{2}, j}}{\delta x}+\frac{\bar{v}_{1} \kappa_{i, j+\frac{1}{2}}-\bar{v}_{2} \kappa_{i, j-\frac{1}{2}}}{\delta y}
$$

em que os termos representados com uma barra são dados por

$$
\bar{u}_{1}=u_{i+\frac{1}{2}, j}, \quad \bar{u}_{2}=u_{i-\frac{1}{2}, j}, \quad \bar{v}_{1}=v_{i, j+\frac{1}{2}} \quad \text { e } \quad \bar{v}_{2}=v_{i, j-\frac{1}{2}} .
$$

Na Eq. (5.13), os valores da propriedade turbulenta $\kappa$ nos pontos $\left(i+\frac{1}{2}, j\right),\left(i-\frac{1}{2}, j\right)$, $\left(i, j+\frac{1}{2}\right)$ e $\left(i, j-\frac{1}{2}\right)$ são aproximados pelo novo esquema upwind de alta resolução EPUS.

Uma vez conhecidas as direções das velocidades advectivas $\bar{u}_{1}, \bar{u}_{2}, \bar{v}_{1}$ e $\bar{v}_{2}$ nas faces $\left(i+\frac{1}{2}, j\right)$, $\left(i+\frac{1}{2}, j\right),\left(i, j+\frac{1}{2}\right)$ e $\left(i, j-\frac{1}{2}\right)$, respectivamente, os pontos vizinhos $\mathbf{D}, \mathbf{U}$ e $\mathbf{R}$, em cada caso, ficam automaticamente especificados. Assim, usando-se a definição (3.2) de variáveis normalizadas de Leonard, seguem as aproximações para as propriedades convectadas nessas faces:

(1) Aproximação para $\kappa_{i+\frac{1}{2}, j}$ :

- Se $\bar{u}_{1}=u_{i+\frac{1}{2}, j}>0$ e $\hat{\kappa}_{U}=\frac{\kappa_{i, j}-\kappa_{i-1, j}}{\kappa_{i+1, j}-\kappa_{i-1, j}}$ então

$$
\kappa_{i+\frac{1}{2}, j}= \begin{cases}\kappa_{R}+\left(\kappa_{D}-\kappa_{R}\right)\left[-4(\lambda-24) \hat{\kappa}_{U}^{8}+16(\lambda-23) \hat{\kappa}_{U}^{7}+(528-25 \lambda) \hat{\kappa}_{U}^{6}+\right. & \\ \left.+(19 \lambda-336) \hat{\kappa}_{U}^{5}+(80-7 \lambda) \hat{\kappa}_{U}^{4}+\lambda \hat{\kappa}_{U}^{3}+\hat{\kappa}_{U}\right], & \text { se } \quad \hat{\kappa}_{U} \in[0,1], \\ \kappa_{U}, & \text { se } \quad \hat{\kappa}_{U} \notin[0,1],\end{cases}
$$

em que

$$
\begin{aligned}
& \mathbf{D}=(i+1, j), \quad \mathbf{U}=(i, j), \quad \mathbf{R}=(i-1, j) ; \\
& - \text { Se } \bar{u}_{1}=u_{i+\frac{1}{2}, j}<0 \text { e } \hat{\kappa}_{U}=\frac{\kappa_{i+1, j}-\kappa_{i+2, j}}{\kappa_{i, j}-\kappa_{i+2, j}} \text { então } \\
& \kappa_{i+\frac{1}{2}, j}= \begin{cases}\kappa_{R}+\left(\kappa_{D}-\kappa_{R}\right)\left[-4(\lambda-24) \hat{\kappa}_{U}^{8}+16(\lambda-23) \hat{\kappa}_{U}^{7}+(528-25 \lambda) \hat{\kappa}_{U}^{6}+\right. & \\
\left.+(19 \lambda-336) \hat{\kappa}_{U}^{5}+(80-7 \lambda) \hat{\kappa}_{U}^{4}+\lambda \hat{\kappa}_{U}^{3}+\hat{\kappa}_{U}\right], & \text { se } \quad \hat{\kappa}_{U} \in[0,1], \\
\kappa_{U}, & \text { se } \quad \hat{\kappa}_{U} \notin[0,1],\end{cases}
\end{aligned}
$$

em que

$$
\mathbf{D}=(i, j), \quad \mathbf{U}=(i+1, j), \quad \mathbf{R}=(i+2, j)
$$


(2) Aproximação para $\kappa_{i-\frac{1}{2}, j}$ :

- Se $\bar{u}_{2}=u_{i-\frac{1}{2}, j}>0$ e $\hat{\kappa}_{U}=\frac{\kappa_{i-1, j}-\kappa_{i-2, j}}{\kappa_{i, j}-\kappa_{i-2, j}}$ então

$\kappa_{i-\frac{1}{2}, j}= \begin{cases}\kappa_{R}+\left(\kappa_{D}-\kappa_{R}\right)\left[-4(\lambda-24) \hat{\kappa}_{U}^{8}+16(\lambda-23) \hat{\kappa}_{U}^{7}+(528-25 \lambda) \hat{\kappa}_{U}^{6}+\right. & \\ \left.+(19 \lambda-336) \hat{\kappa}_{U}^{5}+(80-7 \lambda) \hat{\kappa}_{U}^{4}+\lambda \hat{\kappa}_{U}^{3}+\hat{\kappa}_{U}\right], & \text { se } \hat{\kappa}_{U} \in[0,1], \\ \kappa_{U}, & \text { se } \quad \hat{\kappa}_{U} \notin[0,1],\end{cases}$

em que

$$
\mathbf{D}=(i, j), \quad \mathbf{U}=(i-1, j), \quad \mathbf{R}=(i-2, j) ;
$$

- Se $\bar{u}_{2}=u_{i-\frac{1}{2}, j}<0$ e $\hat{\kappa}_{U}=\frac{\kappa_{i, j}-\kappa_{i+1, j}}{\kappa_{i-1, j}-\kappa_{i+1, j}}$ então

$$
\kappa_{i-\frac{1}{2}, j}= \begin{cases}\kappa_{R}+\left(\kappa_{D}-\kappa_{R}\right)\left[-4(\lambda-24) \hat{\kappa}_{U}^{8}+16(\lambda-23) \hat{\kappa}_{U}^{7}+(528-25 \lambda) \hat{\kappa}_{U}^{6}+\right. & \\ \left.+(19 \lambda-336) \hat{\kappa}_{U}^{5}+(80-7 \lambda) \hat{\kappa}_{U}^{4}+\lambda \hat{\kappa}_{U}^{3}+\hat{\kappa}_{U}\right], & \text { se } \hat{\kappa}_{U} \in[0,1], \\ \kappa_{U}, & \text { se } \quad \hat{\kappa}_{U} \notin[0,1],\end{cases}
$$

em que

$$
\mathbf{D}=(i-1, j), \quad \mathbf{U}=(i, j), \quad \mathbf{R}=(i+1, j) ;
$$

(3) Aproximação para $\kappa_{i, j+\frac{1}{2}}$ :

- Se $\bar{v}_{1}=v_{i, j+\frac{1}{2}}>0$ e $\hat{\kappa}_{U}=\frac{\kappa_{i, j}-\kappa_{i, j-1}}{\kappa_{i, j+1}-\kappa_{i, j-1}}$ então

$$
\kappa_{i, j+\frac{1}{2}}= \begin{cases}\kappa_{R}+\left(\kappa_{D}-\kappa_{R}\right)\left[-4(\lambda-24) \hat{\kappa}_{U}^{8}+16(\lambda-23) \hat{\kappa}_{U}^{7}+(528-25 \lambda) \hat{\kappa}_{U}^{6}+\right. & \\ \left.+(19 \lambda-336) \hat{\kappa}_{U}^{5}+(80-7 \lambda) \hat{\kappa}_{U}^{4}+\lambda \hat{\kappa}_{U}^{3}+\hat{\kappa}_{U}\right], & \text { se } \hat{\kappa}_{U} \in[0,1], \\ \kappa_{U}, & \text { se } \quad \hat{\kappa}_{U} \notin[0,1],\end{cases}
$$

em que

$$
\mathbf{D}=(i, j+1), \quad \mathbf{U}=(i, j), \quad \mathbf{R}=(i, j-1) ;
$$

- Se $\bar{v}_{1}=v_{i, j+\frac{1}{2}}<0$ e $\hat{\kappa}_{U}=\frac{\kappa_{i, j+1}-\kappa_{i, j+2}}{\kappa_{i, j}-\kappa_{i, j+2}}$ então

$$
\kappa_{i, j+\frac{1}{2}}= \begin{cases}\kappa_{R}+\left(\kappa_{D}-\kappa_{R}\right)\left[-4(\lambda-24) \hat{\kappa}_{U}^{8}+16(\lambda-23) \hat{\kappa}_{U}^{7}+(528-25 \lambda) \hat{\kappa}_{U}^{6}+\right. & \\ \left.+(19 \lambda-336) \hat{\kappa}_{U}^{5}+(80-7 \lambda) \hat{\kappa}_{U}^{4}+\lambda \hat{\kappa}_{U}^{3}+\hat{\kappa}_{U}\right], & \text { se } \quad \hat{\kappa}_{U} \in[0,1], \\ \kappa_{U}, & \text { se } \quad \hat{\kappa}_{U} \notin[0,1],\end{cases}
$$

em que

$$
\mathbf{D}=(i, j), \quad \mathbf{U}=(i, j+1), \quad \mathbf{R}=(i, j+2) ;
$$


(4) Aproximação para $\kappa_{i, j-\frac{1}{2}}$ :

- Se $\bar{v}_{2}=v_{i, j-\frac{1}{2}}>0$ e $\hat{\kappa}_{U}=\frac{\kappa_{i, j-1}-\kappa_{i, j-2}}{\kappa_{i, j}-\kappa_{i, j-2}}$ então

$\kappa_{i, j-\frac{1}{2}}= \begin{cases}\kappa_{R}+\left(\kappa_{D}-\kappa_{R}\right)\left[-4(\lambda-24) \hat{\kappa}_{U}^{8}+16(\lambda-23) \hat{\kappa}_{U}^{7}+(528-25 \lambda) \hat{\kappa}_{U}^{6}+\right. & \\ \left.+(19 \lambda-336) \hat{\kappa}_{U}^{5}+(80-7 \lambda) \hat{\kappa}_{U}^{4}+\lambda \hat{\kappa}_{U}^{3}+\hat{\kappa}_{U}\right], & \text { se } \hat{\kappa}_{U} \in[0,1], \\ \kappa_{U}, & \text { se } \quad \hat{\kappa}_{U} \notin[0,1],\end{cases}$

em que

$$
\begin{gathered}
\mathbf{D}=(i, j), \quad \mathbf{U}=(i, j-1), \quad \mathbf{R}=(i, j-2) ; \\
- \text { Se } \bar{v}_{2}=v_{i, j-\frac{1}{2}}<0 \text { e } \hat{\kappa}_{U}=\frac{\kappa_{i, j}-\kappa_{i, j+1}}{\kappa_{i, j-1}-\kappa_{i, j+1}} \text { então } \\
\kappa_{i, j-\frac{1}{2}}=\left\{\begin{array}{c}
\kappa_{R}+\left(\kappa_{D}-\kappa_{R}\right)\left[-4(\lambda-24) \hat{\kappa}_{U}^{8}+16(\lambda-23) \hat{\kappa}_{U}^{7}+(528-25 \lambda) \hat{\kappa}_{U}^{6}+\right. \\
\left.+(19 \lambda-336) \hat{\kappa}_{U}^{5}+(80-7 \lambda) \hat{\kappa}_{U}^{4}+\lambda \hat{\kappa}_{U}^{3}+\hat{\kappa}_{U}\right], \\
\kappa_{U},
\end{array}\right) \text { se } \hat{\kappa}_{U} \in[0,1],
\end{gathered}
$$

em que

$$
\mathbf{D}=(i, j-1), \quad \mathbf{U}=(i, j), \quad \mathbf{R}=(i, j+1)
$$

A aproximação dos demais termos convectivos das equações médias de Navier-Stokes com modelagem $\kappa-\varepsilon$ segue de maneira análoga, assim como as aproximações por meio dos demais esquemas upwind de alta resolução citados no Capítulo 3.

\subsubsection{Metodologia Computacional para as Equações de Navier-Stokes}

No contexto de variáveis primitivas velocidade-pressão, as equações de Navier-Stokes são discretizadas sobre malhas deslocadas. Nessas malhas, variáveis escalares (tais como pressão e energia cinética da turbulência) são avaliadas nos centros das células computacionais, e variáveis vetoriais (velocidade, por exemplo) são avaliadas nas bordas das células computacionais, como visto na Figura 5.1 da Subseção 5.3.1. A metodologia numérica de solução utilizada neste trabalho é a GENSMAC, que é uma variante do método de projeção de Chorin [11], proposto originalmente por Harlow e Welch [23] (método MAC) e bem discutido por Peyret \& Taylor [43]. Baseado no MAC, este método utiliza formulações explícitas e semi-implícitas para a solução numérica de escoamentos incompressíveis não estacionários.

O ambiente de simulação Freeflow (ver [10] e [41]) foi o ambiente computacional adotado para as simulações computacionais de problemas de escoamentos incompressíveis não estacionários. Essa tecnologia numérica adaptada para simular escoamentos em condições adversas foi validada e verificada utilizando-se dados numéricos, experimentais e soluções analíticas. 
A metodologia computacional utilizada na simulação das equações de Navier-Stokes para o caso laminar trata-se de um ciclo computacional que atualiza as variáveis discretas, a partir do tempo inicial $t_{0}$ (ou de um tempo anterior $t_{n}$ ) no tempo $t_{n+1}=t_{n}+\delta t$, utilizando para isso os seguintes passos:

- Passo 1: considera-se $p^{n}=\tilde{p}$ um campo de pressão que satisfaça as condições de contorno na superfície livre, onde a pressão é calculada por meio de (2.35) e o campo de velocidade por meio de (2.36) e (2.37);

- Passo 2: conhecendo-se a pressão, calcula-se um campo de velocidade intermediário $\tilde{u}_{i}$ dado por

$$
\tilde{u}_{i}=u_{i}+\delta_{t}\left[-\frac{\partial\left(u_{i} u_{j}\right)}{\partial x_{j}}-\frac{\partial \tilde{p}}{\partial x_{i}}+\frac{1}{R e} \frac{\partial}{\partial x_{j}}\left(\frac{\partial u_{i}}{\partial x_{j}}\right)+\frac{1}{F r^{2}} g_{i}\right], \quad i=1,2,3
$$

- Passo 3: resolve-se a equação de Poisson para o potencial auxiliar $\varphi$ através de

$$
\frac{\partial}{\partial x_{i}}\left(\frac{\partial \varphi}{\partial x_{i}}\right)=\left(\frac{\partial \tilde{u}_{i}}{\partial x_{i}}\right), \quad i=1,2,3
$$

utilizando-se condição de contorno homogênea de Dirichlet na superfície livre e na saída $(\varphi=0)$, e de Neumann na entrada e no contorno rígido $\left(\frac{\partial \varphi}{\partial n}=0\right)$. Vale ressaltar que o sistema linear (5.16) é resolvido pelo método dos gradientes conjugados (para mais detalhes ver [60]);

- Passo 4: atualiza-se o campo de velocidade através de

$$
u_{i}=\tilde{u}_{i}-\frac{\partial \varphi}{\partial x_{i}}, \quad i=1,2,3
$$

- Passo 5: conhecendo-se o campo de velocidade, atualiza-se o campo de pressão por meio de

$$
p=\tilde{p}+\frac{\varphi}{\delta t}
$$

- Passo 6: determina-se as novas posições das partículas marcadoras que representam o fluido (as quais permitem a visualização do escoamento e a orientação da superfície livre) por meio da resolução do sistema de EDOs

$$
\frac{d x_{i}}{d t}=u_{i}, \quad i=1,2,3
$$

utilizando-se o método de Euler explícito;

- Passo 7: atualiza-se as condições de contorno necessárias e retorna-se ao Passo 1 para dar início a um novo ciclo computacional.

Para o caso turbulento, o ciclo computacional considerado para a resolução das equações 
médias de Navier-Stokes 2D com modelagem $\kappa-\varepsilon$ é análogo ao ciclo computacional apresentado acima para o caso laminar. No entanto alguns passos são acrescentados neste algoritmo para o cálculo das variáveis turbulentas. Para isso, inicia-se o ciclo computacional acima descrito e, após executar o Passo 5, os seguintes passos são executados:

- Passo I: calcula-se a energia cinética turbulenta $\kappa$ através de

$$
\kappa^{n+1}=\kappa^{n}+\delta t\left[-\frac{\partial\left(\kappa u_{j}\right)}{\partial x_{j}}+\frac{1}{R e} \frac{\partial}{\partial x_{j}}\left(\left(1+\frac{\nu_{t}}{\sigma_{\kappa}}\right) \frac{\partial \kappa}{\partial x_{j}}\right)+\nu_{t} D_{i j} \frac{\partial u_{i}}{\partial x_{j}}-\varepsilon\right]
$$

em que $D_{i j}$ é definido pela Eq. (2.42);

- Passo II: calcula-se a dissipação de energia $\varepsilon$ através de

$$
\varepsilon^{n+1}=\varepsilon^{n}+\delta t\left[-\frac{\partial\left(\varepsilon u_{j}\right)}{\partial x_{j}}+\frac{1}{R e} \frac{\partial}{\partial x_{j}}\left(\left(1+\frac{\nu_{t}}{\sigma_{\varepsilon}}\right) \frac{\partial \varepsilon}{\partial x_{j}}\right)+\frac{1}{T_{t}}\left(C_{1 \varepsilon} \nu_{t} D_{i j} \frac{\partial u_{i}}{\partial x_{j}}-C_{2 \varepsilon} \varepsilon\right)\right],
$$

em que $T_{t}, C_{1 \varepsilon}$ e $C_{2 \varepsilon}$ são definidos na Seção 2.5;

- Passo III: atualiza-se a viscosidade turbulenta por

$$
\nu_{t}=C_{\mu} \kappa T_{t}
$$

onde $C_{\mu}$ é definido na Seção 2.5. Calculadas as variáveis $\kappa$ e $\varepsilon$ e a viscosidade turbulenta $\nu_{t}$, completa-se o ciclo computacional executando os passos finais Passo 6 e Passo 7. 
CAPÍTULO

6

\section{Resultados Numéricos 1D}

Com o objetivo de investigar o desempenho do esquema EPUS em problemas 1D, neste capítulo apresentam-se os resultados obtidos por ele na resolução de diversas leis de conservação hiperbólicas 1D, a saber: equação de advecção, Burgers e Euler. Os resultados obtidos são então comparados com resultados de outros esquemas da literatura apresentados na Seção 3.6.

\subsection{Equação Linear de Advecção}

O problema de advecção de um escalar, descrito na Seção 2.1.1, é resolvido nesta seção para quatro diferentes testes numéricos, a saber: no Teste-1 faz-se uma análise do EPUS para diversos valores do parâmetro livre $\lambda$ em problemas com condições iniciais suaves e não suaves; no Teste-2 analisa-se a restrição TVD do novo esquema; e no Teste-3 calcula-se a ordem de convergência observada do EPUS, a qual é comparada com a dos demais esquemas de alta resolução. Na discretização temporal, duas aproximações são consideradas: Euler explícito e Runge-Kutta TVD de terceira ordem. Ainda neste mesmo teste, calcula-se o tempo de CPU do EPUS para resolver o problema proposto, cujos valores são comparados com os obtidos pelos demais esquemas.

Teste-1: conforme demonstrado no Apêndice A, o esquema EPUS satisfaz a restrição de estabilidade TVD para todos os valores de $\lambda$ no intervalo [16,95]. Com o propósito de selecionar alguns destes parâmetros para determinados tipos de problemas, diversos testes numéricos foram realizados (neste trabalho seguem apresentados apenas alguns deles) e uma propriedade importante do novo esquema foi observada: para $\lambda=16$, o esquema EPUS apresenta bons resultados em problemas suaves; enquanto que para $\lambda=95$, melhor desempenho é obtido em problemas com descontinuidades e altos gradientes (problemas não suaves). Para verificar esta propriedade, resolve-se neste teste dois diferentes problemas: Problema Suave e Problema 


\section{Não-Suave.}

- Problema Suave: neste teste avalia-se o esquema EPUS para diferentes valores do parâmetro livre, a saber, $\lambda=16,60$ e 95, sendo estes valores os limitantes do intervalo para $\lambda$ e o outro um valor aleatório no intervalo. Considera-se aqui a equação de advecção suplementada com a condição inicial $u_{0}=\operatorname{sen}(x)$. Na simulação utiliza-se uma malha com $N=100$ células computacionais, número de Courant $\theta=0.5$, tempo final de simulação $t=1.0$ e domínio $x \in[-\pi, \pi]$ $(\delta x=0.06283)$. Os resultados obtidos seguem na Figura 6.1, onde pode-se observar que o novo esquema com $\lambda=16$ captura melhor a solução, enquanto que os resultados obtidos para $\lambda=95$ apresentam pior desempenho. Essas conclusões ficam também evidentes analisando-se a Tabela 6.1, a qual mostra os erros relativos obtidos pelo esquema EPUS para diversos os parâmetros livres.

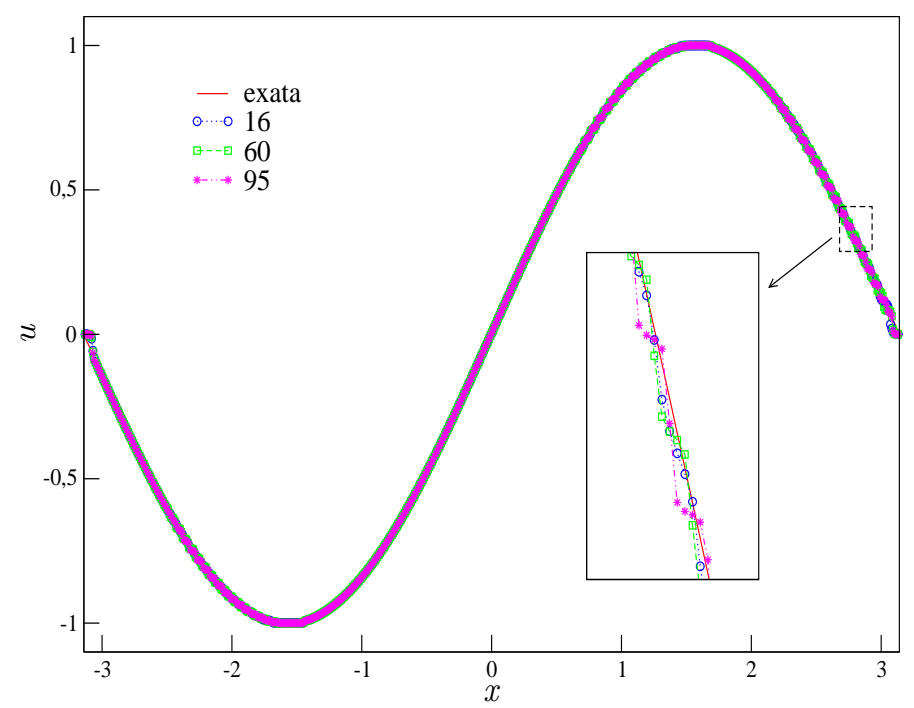

Figura 6.1: comparação para o Teste-1 - Problema Suave de alguns valores do parâmetro livre $\lambda$ para o caso de advecção de um escalar.

\begin{tabular}{l|c|c|c}
\hline \hline EPUS & $\left\|E_{h}\right\|_{1}$ & $\left\|E_{h}\right\|_{2}$ & $\left\|E_{h}\right\|_{\infty}$ \\
\hline$\lambda=16$ & $1.491449 \mathrm{e}-02$ & $1.912056 \mathrm{e}-02$ & $5.468171 \mathrm{e}-02$ \\
\hline$\lambda=60$ & $1.542339 \mathrm{e}-02$ & $2.060312 \mathrm{e}-02$ & $6.170965 \mathrm{e}-02$ \\
\hline$\lambda=95$ & $1.594061 \mathrm{e}-02$ & $2.146075 \mathrm{e}-02$ & $6.792484 \mathrm{e}-02$ \\
\hline \hline
\end{tabular}

Tabela 6.1: erros relativos obtidos pelo EPUS para o Teste-1 - Problema Suave.

- Problema Não-Suave: aqui avalia-se os parâmetros livres do esquema EPUS em um problema não suave. Considera-se a equação de advecção definida em [0,2] e suplementada 
com a seguinte condição inicial:

$$
u_{0}(x)= \begin{cases}\exp \left[-\log (50)\left(\frac{x-0.15}{0.05}\right)^{2}\right], & 0 \leq x<0.2, \\ 1, & 0.3<x<0.4, \\ 20 x-10, & 0.5<x<0.55, \\ 12-20 x, & 0.55 \leq x \leq 0.6, \\ \sqrt{1-\left(\frac{x-0.75}{0.05}\right)^{2}}, & 0.7<x<0.8, \\ 0, & \text { caso contrário. }\end{cases}
$$

Nesta simulação utiliza-se uma malha com $N=100$ células computacionais $(\delta x=0.02)$, número de Courant $\theta=0.5$ e tempo final de simulação $t=0.5$. Os resultados obtidos seguem na Figura 6.2, onde pode-se observar que o novo esquema com $\lambda=95$ captura com melhor desempenho os picos e as regiões de descontinuidades, ao passo que o EPUS com parâmetro $\lambda=16$ suaviza claramente a solução. Essas conclusões ficam também evidentes analisando-se a Tabela 6.2, a qual apresenta os erros relativos obtidos pelo EPUS para os diferentes parâmetros livres.

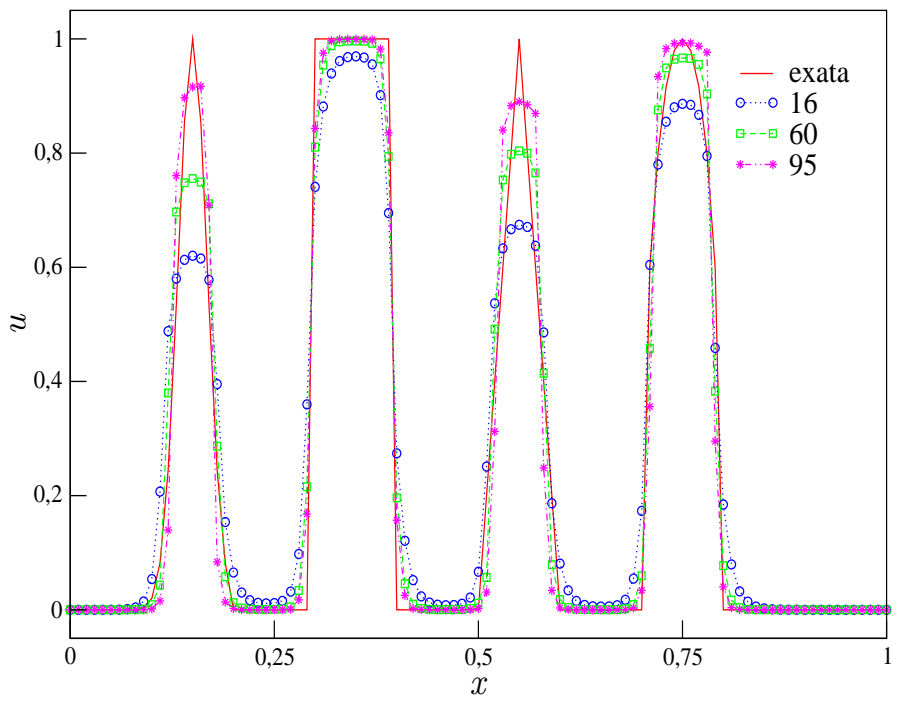

Figura 6.2: comparação para o Teste-1 - Problema Não-Suave de alguns valores do parâmetro livre $\lambda$ para o caso de advecção de um escalar.

Teste-2: neste teste faz-se uma análise da restrição TVD (ver Qamar [45]) para o novo esquema. Para isso, utiliza-se o EPUS com o parâmetro livre $\lambda=95$ aplicado à equação de advecção definida em $[-1,5]$ com condição inicial (problema de Riemann)

$$
u_{0}(x)= \begin{cases}1, & \text { se }-\frac{1}{3} \leq x \leq \frac{1}{3} \\ 0, & \text { caso contrário }\end{cases}
$$




\begin{tabular}{c|ccc}
\hline \hline EPUS & $\left\|E_{h}\right\|_{1}$ & $\left\|E_{h}\right\|_{2}$ & $\left\|E_{h}\right\|_{\infty}$ \\
\hline$\lambda=16$ & $1.533644 \mathrm{e}-01$ & $1.850316 \mathrm{e}-01$ & $3.797700 \mathrm{e}-01$ \\
\hline$\lambda=60$ & $1.394310 \mathrm{e}-01$ & $1.671179 \mathrm{e}-01$ & $3.046600 \mathrm{e}-01$ \\
\hline$\lambda=95$ & $1.153989 \mathrm{e}-01$ & $1.416574 \mathrm{e}-01$ & $2.445100 \mathrm{e}-01$ \\
\hline \hline
\end{tabular}

Tabela 6.2: erros relativos obtidos pelo EPUS para o Teste-1 - Problema Não-Suave.

Nesta simulação, considera-se três diferentes malhas, a saber, $N=50,100$ e 200 células computacionais $(\delta x=0.12,0.06$ e 0.03$)$, número de Courant $\theta=0.8$ e tempo final de simulação $t=4.0$. Os resultados obtidos para esta análise são apresentados na Figura 6.3, em que é possível observar que a TV do esquema EPUS não aumenta conforme avança-se no tempo, satisfazendo, assim, a restrição TVD para estabilidade. É importante ressaltar que, neste trabalho, a estabilidade do esquema EPUS é acessada através de testes numéricos como este. Isso se deve ao fato de que pretende-se aplicar o esquema em problemas não lineares, cuja estabilidade é verificada através de testes numéricos (ver [45]).

(a)

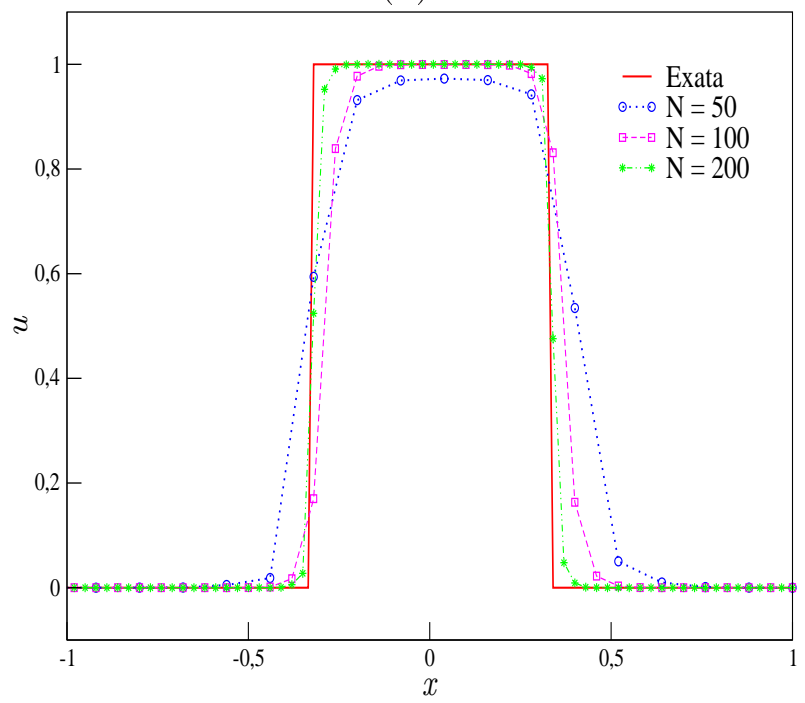

(b)

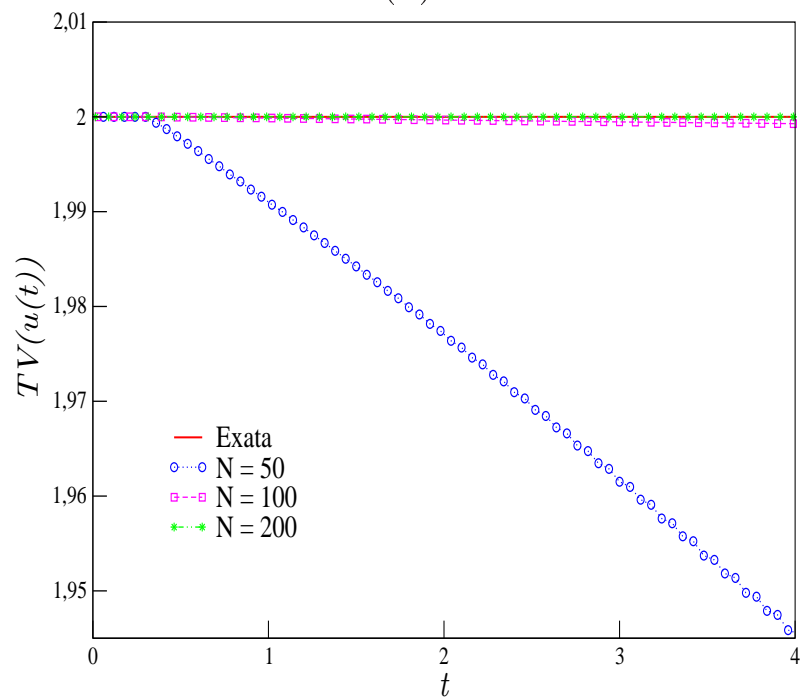

Figura 6.3: análise da TV feita em Teste-2: (a) comparação entre as soluções exata e numéricas e (b) comportamento da TV do esquema EPUS em diferentes malhas.

Teste-3: na busca de uma análise mais quantitativa do esquema EPUS, neste teste calcula-se a ordem de convergência observada, utilizando-se para isto duas aproximações temporais diferentes, a saber, Euler explícito e Runge-Kutta TVD de terceira ordem. Neste caso, a equação de advecção é suplementada com a condição inicial $u_{0}=\operatorname{sen}(\mathrm{x})$. Nesta simulação considera-se malhas com $N=20,40,80,160$ e 320 células computacionais, número de Courant $\theta=0.3$, tempo final de simulação $t=1.0$ e domínio $x \in[-\pi, \pi](\delta x=0.31415,0.15708,0.07854,0.03927 \mathrm{e}$ 
0.01963). Os resultados obtidos para este teste seguem na Tabela 6.3, a qual compara o esquema EPUS com os demais esquemas de alta resolução. A partir de uma análise desta tabela infere-se que o novo esquema mostra-se competitivo com os demais métodos, apresentando maior ordem de convergência quando combinado com Runge-Kutta para a marcha temporal, assim como para os demais esquemas.

\begin{tabular}{|c|c|c|c|c|c|c|c|c|c|}
\hline \multirow[t]{3}{*}{ Esquema } & \multirow{3}{*}{$N$} & \multicolumn{4}{|c|}{ Euler Explícito } & \multicolumn{4}{|c|}{ Runge-Kutta } \\
\hline & & \multicolumn{2}{|c|}{$L_{1}$} & \multicolumn{2}{|c|}{$L_{\infty}$} & \multicolumn{2}{|c|}{$L_{1}$} & \multicolumn{2}{|c|}{$L_{\infty}$} \\
\hline & & $E_{h}$ & $\tilde{p}$ & $E_{h}$ & $\tilde{p}$ & $E_{h}$ & $\tilde{p}$ & $E_{h}$ & $\tilde{p}$ \\
\hline \multirow[t]{5}{*}{ EPUS } & 20 & 0.129743 & - & 0.313360 & - & 0.032687 & - & 0.088860 & - \\
\hline & 40 & 0.051988 & 1.319413 & 0.313340 & 0.000092 & 0.004237 & 2.947580 & 0.029940 & 1.569460 \\
\hline & 80 & 0.012356 & 2.072989 & 0.249430 & 0.329094 & 0.000794 & 2.415378 & 0.016270 & 0.879859 \\
\hline & 160 & 0.003579 & 1.787605 & 0.249820 & -0.002254 & 0.000109 & 2.860303 & 0.008170 & 0.993808 \\
\hline & 320 & 0.001505 & 1.249383 & 0.246570 & 0.018892 & 0.000020 & 2.431090 & 0.004010 & 1.026728 \\
\hline \multirow[t]{5}{*}{ SDPUS-C1 } & 20 & 0.131333 & - & 0.325200 & & 0.032989 & - & 0.075050 & \\
\hline & 40 & 0.055304 & 1.247787 & 0.309710 & 0.070409 & 0.005519 & 2.579637 & 0.031220 & 1.265382 \\
\hline & 80 & 0.013098 & 2.077997 & 0.248910 & 0.315294 & 0.000779 & 2.824350 & 0.016120 & 0.953619 \\
\hline & 160 & 0.003194 & 2.035910 & 0.249880 & -0.005611 & 0.000144 & 2.438288 & 0.008090 & 0.994640 \\
\hline & 320 & 0.001502 & 1.088688 & 0.247440 & 0.014157 & 0.000023 & 2.636047 & 0.003980 & 1.023371 \\
\hline \multirow[t]{5}{*}{ TOPUS } & 20 & 0.125627 & - & 0.300040 & & 0.041857 & - & 0.092270 & - \\
\hline & 40 & 0.049530 & 1.342779 & 0.250000 & 0.263227 & 0.007626 & 2.456376 & 0.035510 & 1.377636 \\
\hline & 80 & 0.013048 & 1.924508 & 0.250000 & 0.000000 & 0.000964 & 2.984664 & 0.016110 & 1.140269 \\
\hline & 160 & 0.002698 & 2.274025 & 0.250000 & 0.000000 & 0.000195 & 2.301580 & 0.008000 & 1.009885 \\
\hline & 320 & 0.001252 & 1.107132 & 0.250000 & 0.000000 & 0.000027 & 2.860690 & 0.003950 & 1.018147 \\
\hline \multirow[t]{5}{*}{ ADB } & 20 & 0.023845 & - & 0.121090 & - & 0.129623 & - & 0.205110 & \\
\hline & 40 & 0.006465 & 1.882878 & 0.135750 & -0.164872 & 0.036191 & 1.840599 & 0.111830 & 0.875091 \\
\hline & 80 & 0.002068 & 1.644383 & 0.142170 & -0.066665 & 0.009552 & 1.921738 & 0.058580 & 0.932828 \\
\hline & 160 & 0.000974 & 1.086607 & 0.144460 & -0.023053 & 0.002452 & 1.962065 & 0.030050 & 0.963043 \\
\hline & 320 & 0.000458 & 1.087803 & 0.144820 & -0.003591 & 0.000621 & 1.982272 & 0.015220 & 0.981396 \\
\hline \multirow[t]{5}{*}{ WACEB } & 20 & 0.139407 & - & 0.345490 & - & 0.033652 & - & 0.141521 & - \\
\hline & 40 & 0.052389 & 1.411979 & 0.354650 & -0.037752 & 0.004799 & 2.809938 & 0.055704 & 1.345154 \\
\hline & 80 & 0.012282 & 2.092712 & 0.250000 & 0.504468 & 0.000906 & 2.404461 & 0.027775 & 1.004018 \\
\hline & 160 & 0.003595 & 1.772582 & 0.250000 & 0.000000 & 0.000173 & 2.386474 & 0.013379 & 1.053760 \\
\hline & 320 & 0.001374 & 1.387510 & 0.245540 & 0.025970 & 0.000036 & 2.274018 & 0.006373 & 1.069952 \\
\hline \multirow[t]{5}{*}{ CUBISTA } & 20 & 0.130729 & 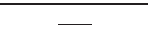 & 0.317830 & & 0.043900 & - & 0.162302 & - \\
\hline & 40 & 0.067099 & 0.962211 & 0.330870 & -0.058009 & 0.006595 & 2.734775 & 0.056310 & 1.527209 \\
\hline & 80 & 0.010881 & 2.624474 & 0.249920 & 0.404798 & 0.001053 & 2.646613 & 0.028006 & 1.007677 \\
\hline & 160 & 0.003380 & 1.686626 & 0.247480 & 0.014154 & 0.000204 & 2.365172 & 0.013475 & 1.055463 \\
\hline & 320 & 0.001594 & 1.084309 & 0.238680 & 0.052234 & 0.000039 & 2.404677 & 0.006411 & 1.071564 \\
\hline
\end{tabular}

Tabela 6.3: erros relativos nas normas $L_{1}$ e $L_{\infty}$ e ordem de convergência $(\tilde{p})$ calculados para o Teste-3 utilizando como aproximação temporal Euler explícito e Runge-Kutta TVD de terceira ordem.

Neste mesmo teste, faz-se uma outra análise quantitativa, em que se calcula o tempo de CPU utilizado pelos esquemas para resolver o problema aqui proposto. Nesta simulação considera-se uma malha com $N=400$ células computacionais, número de Courant $\theta=0.3$ e 0.5 , tempos finais de simulação $t=10.0$ e 20.0, no domínio $x \in[-\pi, \pi](\delta x=0.01571)$. Os resultados obtidos para este teste seguem apresentados na Tabela 6.4, em que é possível observar que o 
esquema EPUS, comparado com os demais esquemas polinomiais (SDPUS-C1 e TOPUS), é um pouco mais caro computacionalmente, como era esperado, considerando que o novo esquema é dado por um polinômio de grau oito, enquanto os outros dois são dados por polinômios de grau seis e quatro, respectivamente. No entanto, esse é o preço a se pagar para obter melhores resultados (principalmente em malhas grosseiras, onde o EPUS mostrou-se melhor). Comparado ao esquema ADBQUICKEST, o EPUS é mais barato por apresentar menos condicionais em sua formulação.

\begin{tabular}{l|rc|cc}
\hline \hline \multirow{2}{*}{ Esquema } & \multicolumn{2}{|c|}{$t=10$} & \multicolumn{2}{c}{$t=20$} \\
\cline { 2 - 5 } & $\theta=0.3$ & $\theta=0.5$ & $\theta=0.3$ & $\theta=0.5$ \\
\hline EPUS & 2.14 & 1.22 & 3.46 & 2.00 \\
\hline SDPUS-C1 & 2.07 & 1.18 & 3.38 & 1.95 \\
\hline TOPUS & 1.99 & 1.04 & 3.32 & 1.82 \\
\hline ADBQUICKEST & 2.26 & 1.31 & 3.58 & 2.08 \\
\hline \hline
\end{tabular}

Tabela 6.4: tempo de CPU calculado para o Teste-3.

\subsection{Equação Não-Linear de Burgers}

Nesta seção, a equação de Burgers, descrita na Seção 2.1.2, é solucionada em quatro situações (condições iniciais) com o objetivo de verificar se o esquema EPUS é apropriado para este problema não linear. No Teste-4 faz-se uma análise para diversos valores do parâmetro livre $\lambda$ em problemas com condições iniciais suaves e não suaves; no Teste- 5 analisa-se a restrição TVD do esquema EPUS; no Teste-6, para melhor avaliar o novo esquema, calcula-se sua ordem de convergência, a qual é comparada com a dos demais esquemas de alta resolução; e no Teste-7 faz-se uma comparação qualitativa dos esquemas para um problema não suave, o qual foi implementado utilizando-se a técnica de correção de entropia (ver [24, 55]).

Teste-4: a partir deste teste, faz-se uma análise do parâmetro livre $\lambda$, assim como feito para a equação de advecção, ilustrando novamente, agora para o caso não linear, que o EPUS com parâmetro livre $\lambda=16$ apresenta os melhores resultados para problemas suaves, enquanto que para o parâmetro $\lambda=95$, melhores resultados são alcançados em problemas não suaves.

- Problema Suave: neste teste, a equação de Burgers é aplicada à condição inicial $u_{0}=\operatorname{sen}(\mathrm{x})$ para $x \in[0,2 \pi]$. A solução exata para este problema é dada por (ver Platzman [44])

$$
u(x, t)=-2 \sum_{n}^{\infty} \frac{J_{n}(-n t)}{n t} \operatorname{sen}(n x),
$$

em que $J_{n}$ é a função de Bessel de grau $n$. Considera-se a equação (6.3) truncada com $n=500$ 
termos. Para a simulação, utiliza-se uma malha com $N=200$ células computacionais ( $\delta x=$ 0.03141 ), número de Courant $\theta=0.5$ e tempo final de simulação $t=1.0$. Aqui, os valores $\lambda=16,60$ e 95 são testados e, conforme esperado, os melhores resultados são obtidos pelo EPUS $\operatorname{com} \lambda=16$, como mostra a Figura 6.4. Vê-se por essa figura que menos oscilações são formadas ao aplicar o esquema com este parâmetro. Com o objetivo de ilustrar quantitativamente esta análise, apresenta-se a Tabela 6.5, a qual mostra o erro relativo obtido neste teste e comprova o bom desempenho do esquema EPUS com parâmetro $\lambda=16$.
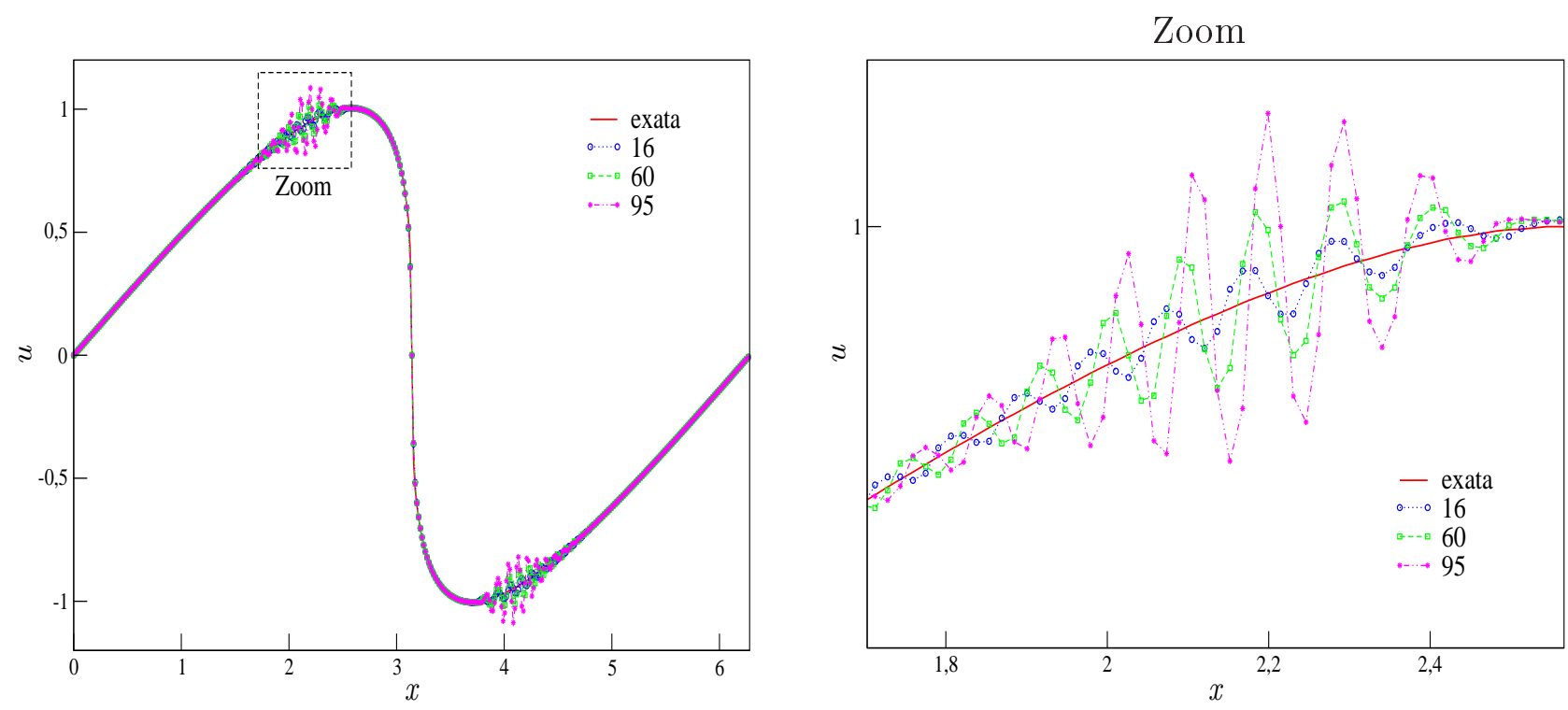

Figura 6.4: comparação para o Teste-4 - Problema Suave de alguns valores do parâmetro livre $\lambda$ para o caso não linear.

\begin{tabular}{c|c|c|c}
\hline \hline EPUS & $\left\|E_{h}\right\|_{1}$ & $\left\|E_{h}\right\|_{2}$ & $\left\|E_{h}\right\|_{\infty}$ \\
\hline$\lambda=16$ & $7.145545 \mathrm{e}-03$ & $1.286337 \mathrm{e}-02$ & $7.837454 \mathrm{e}-02$ \\
\hline$\lambda=60$ & $1.235676 \mathrm{e}-02$ & $2.292389 \mathrm{e}-02$ & $8.081938 \mathrm{e}-02$ \\
\hline$\lambda=95$ & $2.041206 \mathrm{e}-02$ & $4.192552 \mathrm{e}-02$ & $1.381012 \mathrm{e}-01$ \\
\hline \hline
\end{tabular}

Tabela 6.5: erros relativos obtidos pelo EPUS para o Teste-4 - Problema Suave.

- Problema Não-Suave: agora, assim como feito para a equação de advecção, ilustra-se a propriedade de o esquema EPUS apresentar melhor comportamento com o parâmetro livre $\lambda=95$ para problemas não suaves. Para isso, considera-se a equação de Burgers suplementada 
com condição inicial

$$
u_{0}(x)=\left\{\begin{array}{lcc}
1, & \text { se } & x \leq 1.5 \\
2.5-x, & \text { se } & 1.5<x \leq 2.5 \\
0, & \text { se } & x>2.5
\end{array}\right.
$$

cuja solução exata é (ver Shin et al. [49]):

- Para $t<1$ :

$$
u(x, t)=\left\{\begin{array}{llc}
1, & \text { se } & x \leq 1.5+t \\
\frac{2.5-x}{1-t}, & \text { se } & 1.5+t<x \leq 2.5, \\
0, & \text { se } & x>2.5
\end{array}\right.
$$

- Para $t>1$ :

$$
u(x, t)=\left\{\begin{array}{lll}
1, & \text { se } & x \leq 2+0.5 t \\
0, & \text { se } & x>2+0.5 t
\end{array}\right.
$$

Neste teste, consideram-se uma malha com $N=200$ células computacionais, número de Courant $\theta=0.5$, tempo final de simulação $t=1.0$ e domínio $x \in[1,3.5](\delta x=0.0125)$. Os resultados obtidos são apresentados nas Figuras 6.5 e 6.6, em que pode-se observar que o EPUS com parâmetro livre $\lambda=95$ apresenta os melhores resultados neste problema com descontinuidade. Com o objetivo de ilustrar quantitativamente esta análise, apresenta-se a Tabela 6.6, a qual mostra o erro relativo obtido neste teste, comprovando que o esquema EPUS com parâmetro $\lambda=95$ apresenta melhor desempenho neste caso não suave.

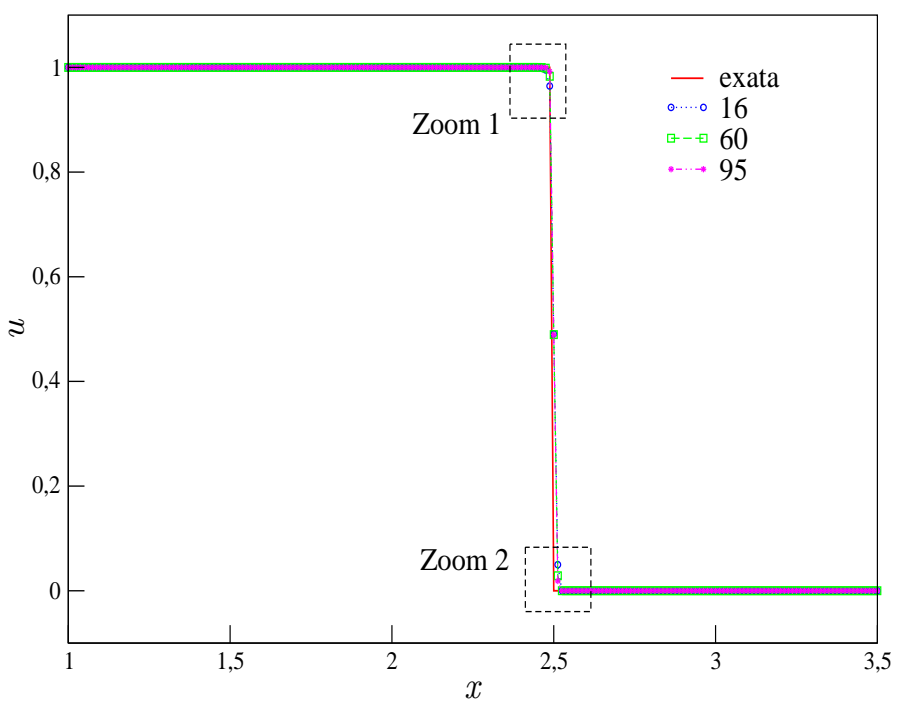

Figura 6.5: comparação para o Teste-4 - Problema Não-Suave de alguns valores do parâmetro livre $\lambda$ para o caso não linear. 
Zoom 1

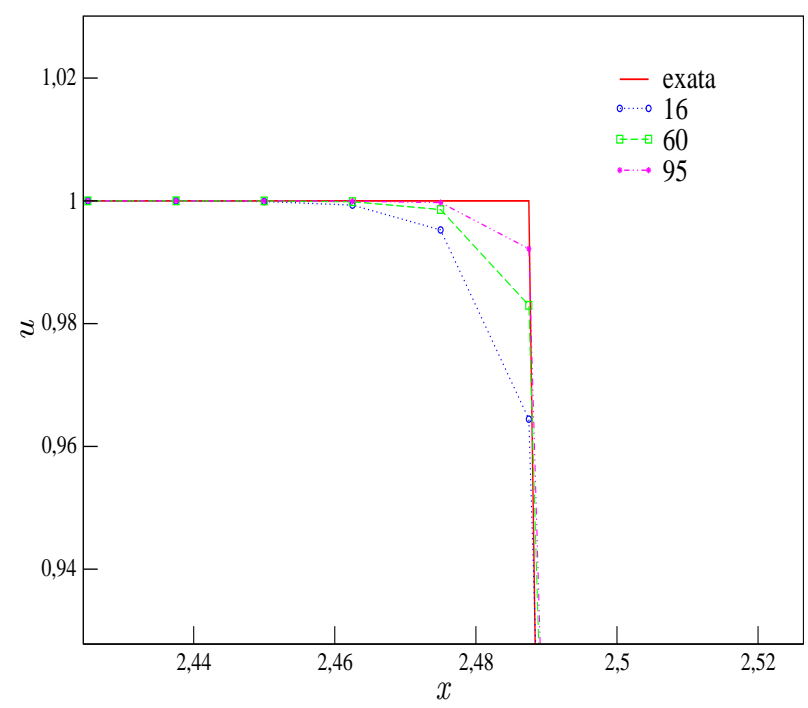

Zoom 2

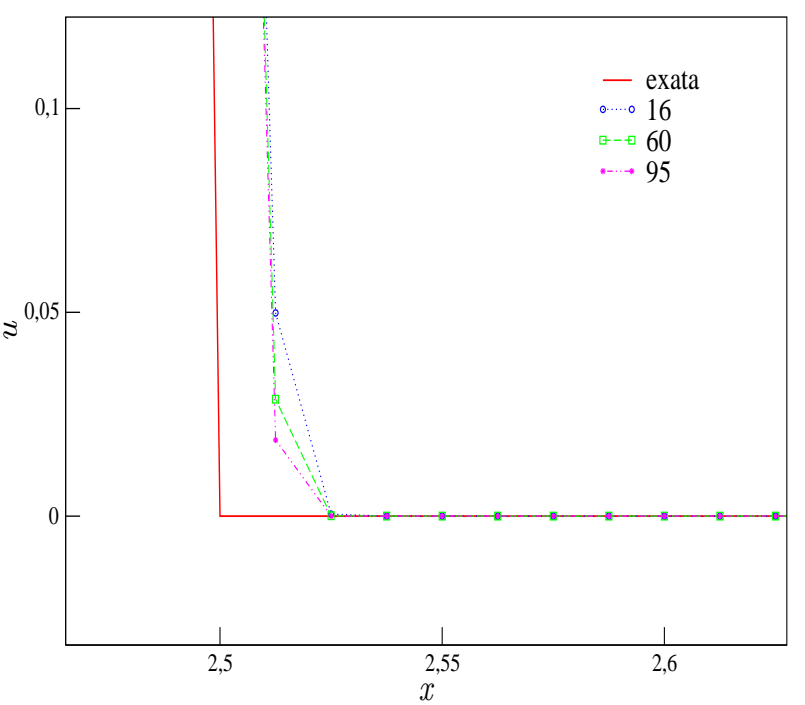

Figura 6.6: regiões de ampliação da Figura 6.5.

\begin{tabular}{l|c|c|c}
\hline \hline EPUS & $\left\|E_{h}\right\|_{1}$ & $\left\|E_{h}\right\|_{2}$ & $\left\|E_{h}\right\|_{\infty}$ \\
\hline$\lambda=16$ & $1.548996 \mathrm{e}-04$ & $1.559159 \mathrm{e}-03$ & $1.701999 \mathrm{e}-02$ \\
\hline$\lambda=60$ & $3.426671 \mathrm{e}-04$ & $3.275180 \mathrm{e}-03$ & $3.555602 \mathrm{e}-02$ \\
\hline$\lambda=95$ & $6.784996 \mathrm{e}-05$ & $7.129032 \mathrm{e}-04$ & $7.803977 \mathrm{e}-03$ \\
\hline \hline
\end{tabular}

Tabela 6.6: erros relativos obtidos pelo EPUS para o Teste-4 - Problema Não-Suave.

Teste-5: neste teste faz-se uma análise da propriedade TVD para o esquema EPUS, utilizando para isto a equação de Burgers com condição inicial e respectiva solução exata dadas por (ver Qamar [45] e Kumar [27])

$$
u_{0}(x)=\left\{\begin{array}{ll}
1, & \text { se }|x|<\frac{1}{3}, \\
0, & \text { se } \frac{1}{3}<|x| \leq 1,
\end{array} \quad \text { e } \quad u(x, t)=\left\{\begin{array}{cl}
0, & \text { se } x<-\frac{1}{3}, \\
\frac{x+\frac{1}{3}}{t}, & \text { se }-\frac{1}{3}<x<t-\frac{1}{3}, \\
1, & \text { se } t-\frac{1}{3}<x<\frac{1}{2} t+\frac{1}{3} \\
0, & \text { se } x>\frac{1}{2} t+\frac{1}{3} .
\end{array}\right.\right.
$$

Para a simulação deste teste, utiliza-se 3 malhas computacionais, a saber, $N=50,100$ e 200 , número de Courant $\theta=0.5$ e tempo final de simulação $t=0.6$, no domínio $x \in[-1,1]$ $(\delta x=0.04,0.02$ e 0.01$)$. Os resultados aqui obtidos seguem apresentados na Figura 6.7, em que vê-se claramente que o esquema EPUS satisfaz a propriedade TVD de estabilidade. Em particular, observa-se na Figura 6.7 (a) que houve convergência monótona da solução numérica. É importante ressaltar que, assim como no caso da equação de advecção, aqui a estabilidade do esquema é acessada através de testes numéricos, pelo fato de que pretende-se testar o esquema 
EPUS em problemas não lineares.

(a)

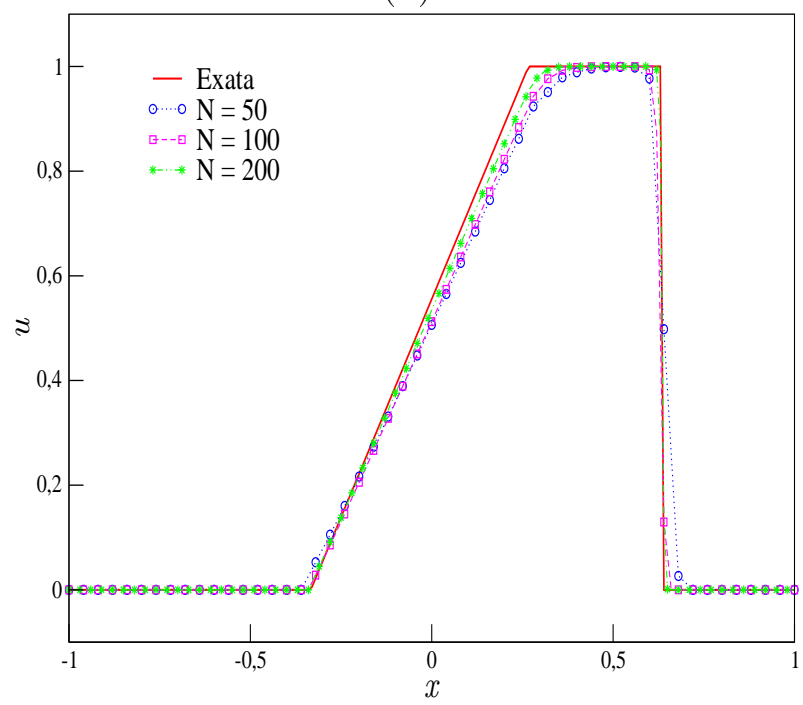

(b)

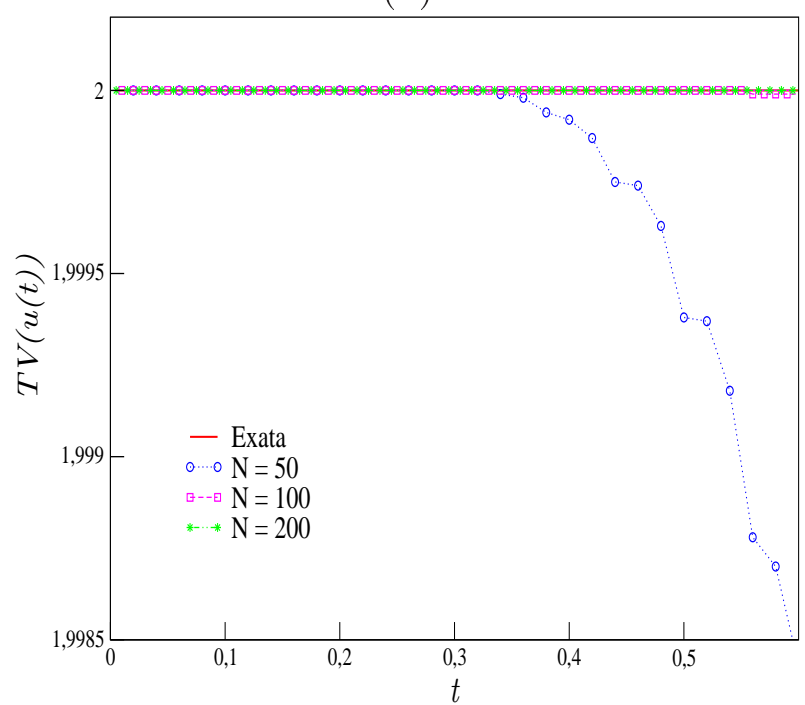

Figura 6.7: análise da TV feita no Teste-5: (a) comparação entre as soluções exata e numéricas e (b) comportamento da TV do esquema EPUS em diferentes malhas.

Teste-6: na busca de uma análise quantitativa do esquema EPUS, neste teste acessa-se sua ordem de convergência bem como comparações com outros esquemas. Considera-se o mesmo problema apresentado no Teste-1, utilizando-se os seguintes dados: malhas computacionais $N=$ $20,40,80,160$ e $320, \theta=0.5, t=0.25$ e domínio $x \in[-\pi, \pi](\delta x=0.31415,0.15708,0.07854$, 0.03927 e 0.01963). A comparação dos resultados é apresentada na Tabela 6.7, a partir da qual pode-se concluir que, em geral, os melhores resultados são obtidos com o esquema EPUS. Observa-se também que os esquemas ADBQUICKEST e SUPERBEE apresentam ordem de convergência inferiores àquelas dos demais esquemas, sendo que na norma infinito a ordem observada foi negativa. Analisando-se também os erros relativos, infere-se que o esquema WACEB, apesar de não apresentar a maior ordem de convergência, é aquele que apresenta menor erro relativo. Já o esquema EPUS, dentre os esquemas polinomiais, é aquele que apresenta maior erro relativo na primeira malha, mas com erro relativo menor na última malha, o que era de se esperar pelo fato de apresentar maior ordem de convergência. 


\begin{tabular}{l|r|cc|cc}
\hline \hline Esquema & \multicolumn{1}{|c|}{$N$} & \multicolumn{2}{|c|}{$L_{1}$} & \multicolumn{2}{c}{$L_{\infty}$} \\
\cline { 3 - 6 } & & $E_{h}$ & $\tilde{p}$ & $E_{h}$ & $\tilde{p}$ \\
\hline EPUS & 20 & 0.078897 & - & 0.033576 & - \\
& 40 & 0.017333 & 2.186443 & 0.011631 & 1.529454 \\
& 80 & 0.005186 & 1.740887 & 0.005483 & 1.084940 \\
& 160 & 0.000692 & 2.906027 & 0.001605 & 1.772381 \\
& 320 & 0.000314 & 1.139525 & 0.001578 & 0.024476 \\
\hline SDPUS & 20 & 0.072521 & - & 0.024405 & - \\
& 40 & 0.016754 & 2.113902 & 0.009341 & 1.385533 \\
& 80 & 0.004611 & 1.861411 & 0.005356 & 0.802423 \\
& 160 & 0.000653 & 2.820935 & 0.001615 & 1.729599 \\
& 320 & 0.000317 & 1.040141 & 0.001580 & 0.031646 \\
\hline TOPUS & 20 & 0.070807 & - & 0.026128 & - \\
& 40 & 0.017313 & 2.032027 & 0.008982 & 1.540492 \\
& 80 & 0.004611 & 1.908829 & 0.005398 & 0.734609 \\
& 160 & 0.000668 & 2.787919 & 0.001612 & 1.743591 \\
& 320 & 0.000317 & 1.075243 & 0.001579 & 0.029804 \\
\hline ADBQUICKEST & 20 & 4.485276 & - & 1.444864 & - \\
& 40 & 2.238254 & 1.002823 & 1.456373 & -0.011446 \\
& 80 & 1.118915 & 1.000274 & 1.451798 & 0.004539 \\
& 160 & 0.559377 & 1.000207 & 1.455630 & -0.003803 \\
& 320 & 0.279677 & 1.000059 & 1.456201 & -0.000565 \\
\hline WACEB & 20 & 0.069035 & - & 0.021577 & - \\
& 40 & 0.016246 & 2.087238 & 0.009577 & 1.171848 \\
& 80 & 0.004453 & 1.867334 & 0.005334 & 0.844363 \\
& 160 & 0.000651 & 2.773919 & 0.001617 & 1.721902 \\
& 320 & 0.000315 & 1.047434 & 0.001580 & 0.033404 \\
\hline SUPERBEE & 20 & 4.487269 & - & 1.460789 & - \\
& 40 & 2.238511 & 1.003299 & 1.465684 & -0.004826 \\
& 80 & 1.118840 & 1.000535 & 1.453763 & 0.011781 \\
& 160 & 0.559342 & 1.000202 & 1.456630 & -0.002843 \\
& 320 & 0.279667 & 1.000020 & 1.456739 & -0.000108 \\
\hline \hline
\end{tabular}

Tabela 6.7: erros relativos nas normas $L_{1}$ e $L_{\infty}$ e estimativa para ordem de convergência $(\tilde{p})$ calculados para o Teste-6.

Teste-7: embora atenção especial seja dada pela comunidade científica em CFD para a captura da solução nas regiões de ondas de choques e altos gradientes, dificuldades numéricas aparecem na presença de ondas de rarefação, sendo uma delas a imprecisão no ponto sônico (ponto em que a velocidade da onda muda de sinal), em que um máximo e/ou um mínimo local são criados e a solução deixa de ser monótona ao longo da onda de expansão.

Segundo Tang [55], esquemas TVD podem apresentar esses problemas próximo ao ponto sônico (que para a equação de Burgers é em $u=0$ ). Ainda, este autor afirma que isso também pode ocorrer com esquemas que utilizam a estratégia upwind. Uma técnica eficiente para resolver este fenômeno á apresentada por Tang [55], conhecida como correção de entropia (no caso, é utilizada a correção de entropia de Harten [24]), a qual introduz uma viscosidade numérica no esquema de diferenças finitas.

Para a introdução desse efeito, considera-se um esquema geral de três pontos da forma 
(expressão 5.1 com termo adicional):

$$
\phi_{i}=\phi_{i}-\theta\left(F_{i+1}-F_{i-1}\right)+\frac{Q_{i+\frac{1}{2}}}{2}\left(\phi_{i+1}-\phi_{i}\right)-\frac{Q_{i-\frac{1}{2}}}{2}\left(\phi_{i}-\phi_{i-1}\right)
$$

em que o termo $Q_{i+\frac{1}{2}}$ é dado, neste caso, pela correção de entropia de Harten, da forma:

$$
Q(x)= \begin{cases}|x|, & |x|>\varepsilon \\ \frac{x^{2}+\varepsilon^{2}}{2 \varepsilon}, & |x| \leq \varepsilon\end{cases}
$$

onde $\varepsilon$ é uma constante positiva dada.

Para ilustrar esse curioso fenômeno e mostrar como ele é solucionado através da correção de entropia, suplementa-se a equação de Burgers com a condição inicial

$$
u_{0}(x)=\left\{\begin{aligned}
1, & \text { se }|x|<\frac{1}{3} \\
-1, & \text { se } \frac{1}{3}<|x| \leq 1
\end{aligned}\right.
$$

cuja solução exata é

$$
u(x, t)=\left\{\begin{array}{cl}
-1, & \text { se } x<-t-\frac{1}{3} \\
\frac{x+\frac{1}{3}}{t}, & \text { se }-t-\frac{1}{3}<x<t-\frac{1}{3} \\
1, & \text { se } t-\frac{1}{3}<x<\frac{1}{3} \\
-1, & \text { se } x>\frac{1}{3}
\end{array}\right.
$$

Para a simulação deste problema, utiliza-se uma malha com $N=400$ células computacionais, $\theta=0.5, t=0.3$, domínio computacional $x \in[-1,1](\delta x=0.005)$ e considera-se como escolha para a constante positiva da correção de entropia $\varepsilon=0.5$ (escolha essa feita a partir de diversos testes numéricos).

Os resultados obtidos para este teste seguem apresentados na Figura 6.8 para vários esquemas, a qual apresenta uma comparação entre as soluções analítica e numéricas (estas calculadas sem e com a correção de entropia). Vê se claramente por esta figura que os resultados numéricos obtidos pelos esquemas sem a correção de entropia apresentam efeito dispersivo (não monótono) próximo ao ponto sônico, imprecisão essa que é solucionada pela adição da correção de entropia no esquema. Neste teste, observa-se que todos os esquemas capturam a solução quando há a correção de entropia, mas pode-se inferir que os polinomiais apresentam melhor desempenho com relação ao ADBQUICKEST, que é um esquema linear por partes. 

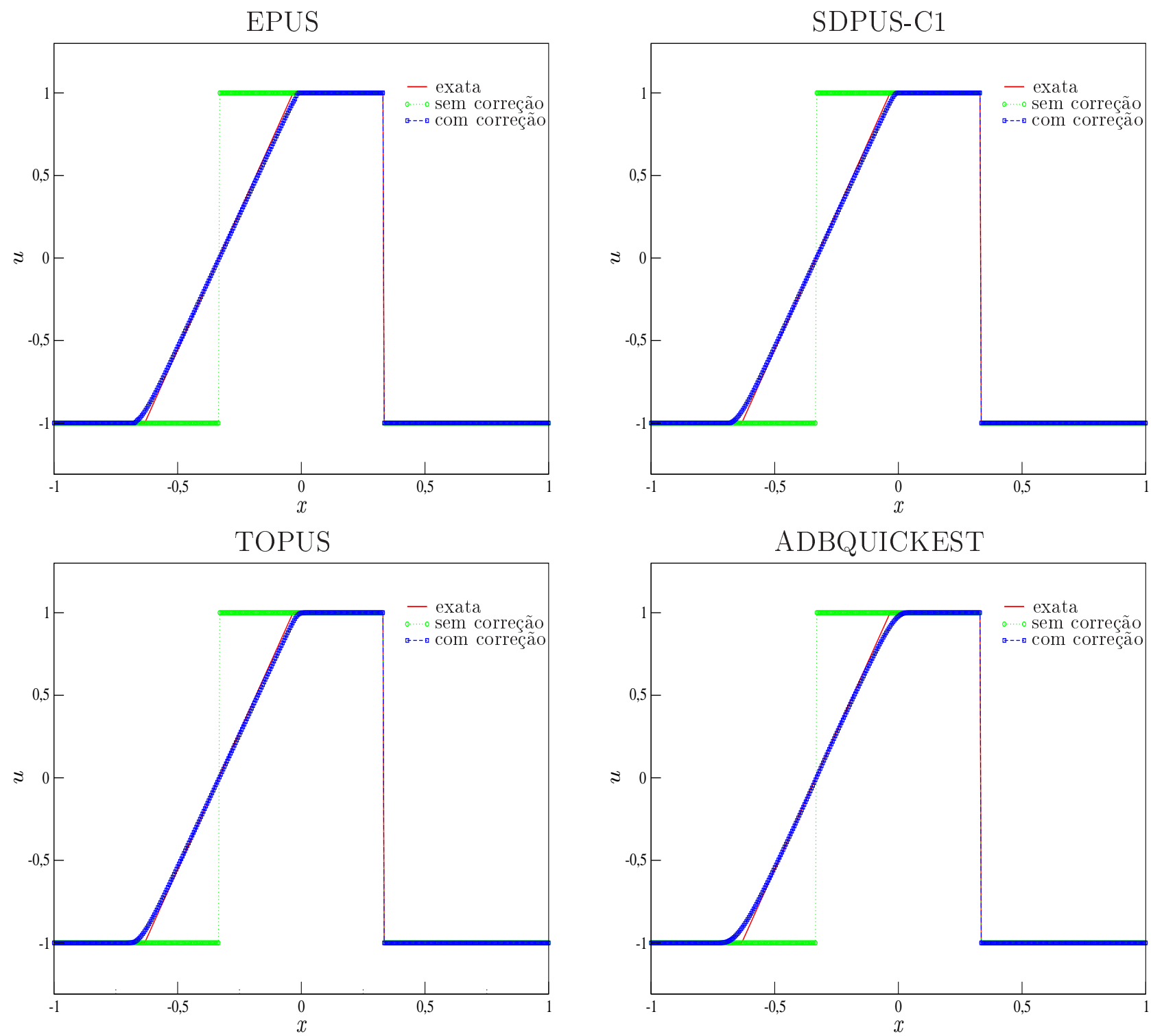

Figura 6.8: ilustração dos resultados obtidos sem e com correção de entropia para o Teste-7.

\subsection{Equações Não-Lineares de Euler}

Este sistema de equações não lineares está descrito na Seção 2.1.3. Para estas equações analisou-se o desempenho do esquema EPUS em resolver problemas de tubos de choques, comparando-o com o desempenho dos demais esquemas de alta resolução. Para esta análise, dois testes são apresentados, a saber: no Teste- 8 resolve-se o famoso problema de tubo de choque Two Interacting Blast Waves (por Wood e Collela [68]), conhecido por apresentar fortes interações de choques. Ainda neste teste faz-se uma avaliação qualitativa de seu desempenho, comparando tempo de CPU, erro relativo e ordem de convergência; e no Teste-9 simula-se o 
problema do tubo de choque de Sod [51], em que analisa-se qualitativamente o desempenho dos esquemas.

Teste-8: neste teste, simula-se o famoso problema Two Interacting Blast Waves proposto por Wood e Collela [68]. É um problema muito interessante pelo fato de apresentar fortes interações entre choques, além de ondas de rarefações e ondas de contato. Os dados iniciais consistem em duas ondas de choques fortes propagando em direções opostas e que colidem uma com a outra. Sua condição inicial depende de três estados constantes da forma

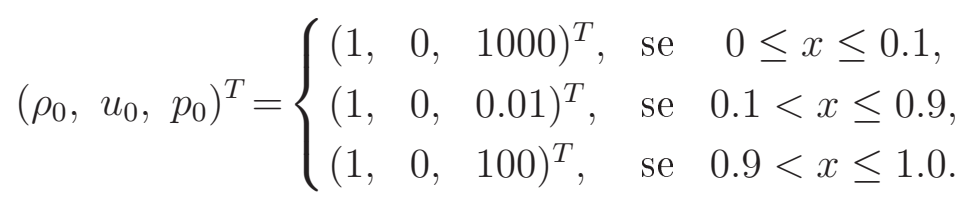

Para esta simulação considera-se uma malha com $N=1000$ células computacionais, número de Courant $\theta=0.9$, tempo final $t=0.038$ e domínio $x \in[0,1](\delta x=0.001)$. Para a solução de referência, os valores são calculados com o limitador de fluxo MC em uma malha com $N=2000$ células computacionais. Os resultados obtidos para este teste são mostrados nas Figuras 6.9, 6.10 e 6.11, em que pode-se observar que o esquema EPUS captura o fenômeno satisfatoriamente, apresentando melhor desempenho que os demais esquemas polinomiais. No entanto, em algumas regiões, o esquema ADBQUICKEST foi o que se comportou melhor.

Considerando o quão reconhecido é este problema, faz-se neste teste uma avaliação quantitativa do esquema EPUS. Para isso seu erro relativo e ordem de convergência são calculados, os quais são comparados com os obtidos pelos demais esquemas de alta resolução. Ainda, calcula-se o tempo de CPU utilizado por cada esquema para resolver este problema. Foram consideradas 4 malhas computacionais, a saber, $N=125,250,500$ e 1000. Os resultados obtidos seguem apresentados na Tabela 6.8, em que observa-se que o novo esquema foi o que alcançou maior ordem de convergência e também o menor erro relativo dentre todos os esquemas apresentados. No entanto, por ser derivado de um polinômio de grau oito, não é o que apresenta menor tempo de CPU para a execução deste teste. Levando em conta que bons resultados foram obtidos pelo EPUS, considera-se que o tempo de CPU utilizado por ele seja aceitável, sendo este o preço a se pagar para obter melhores resultados. 


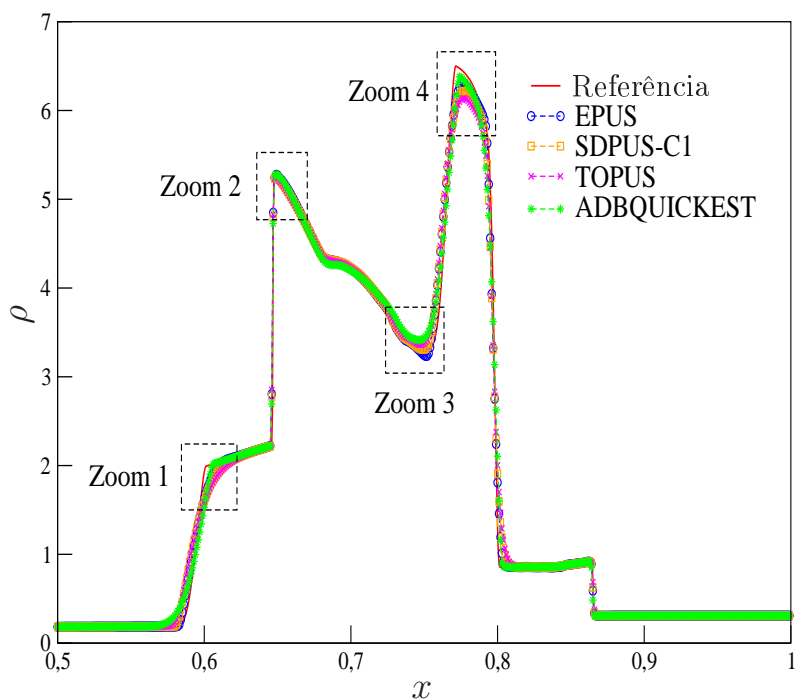

\section{Zoom 1}

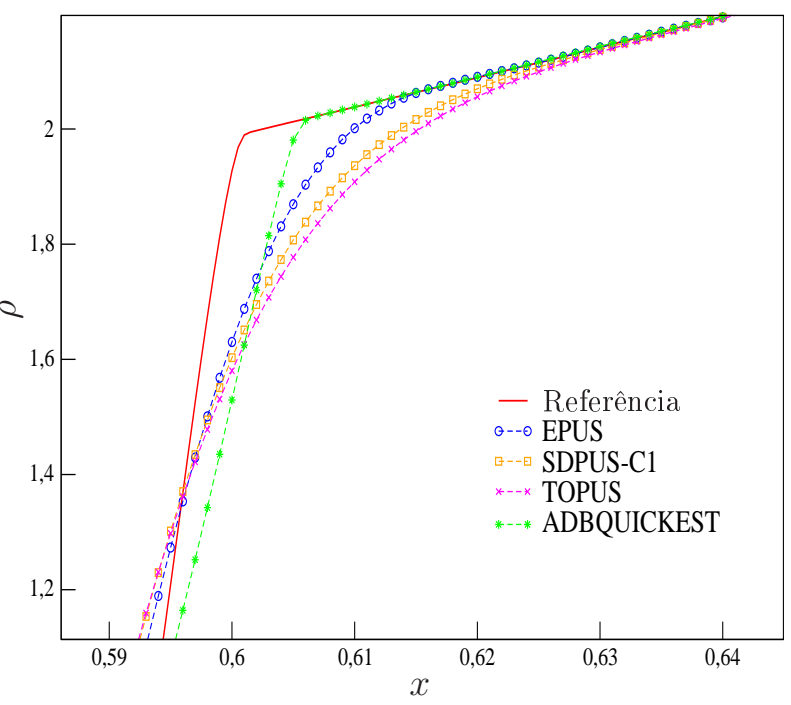

Zoom 3

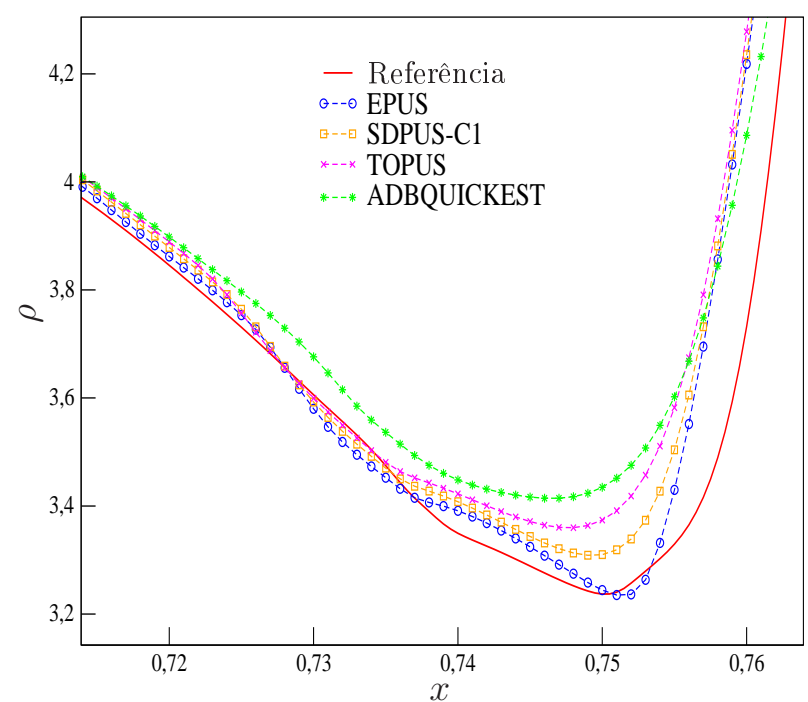

Zoom 2

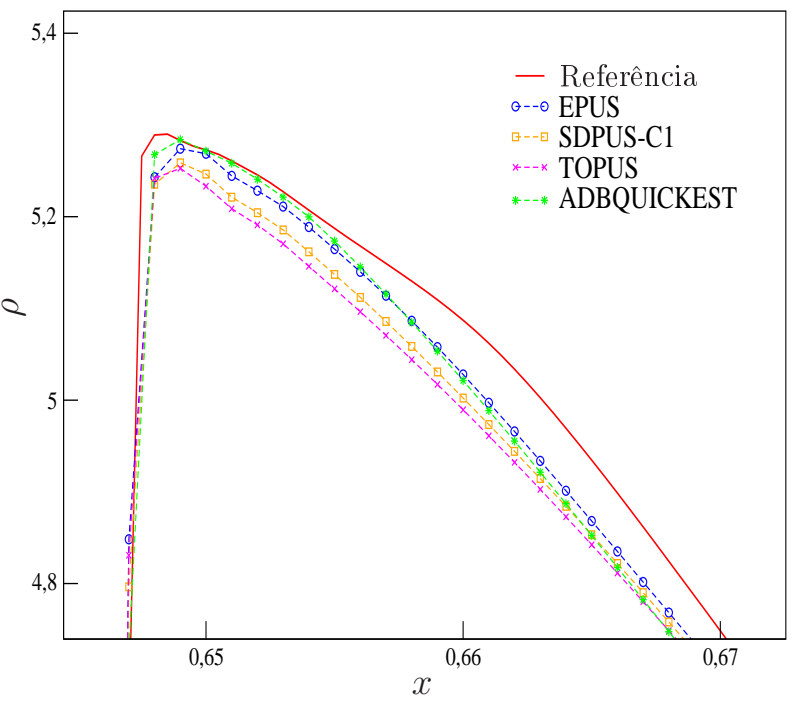

Zoom 4

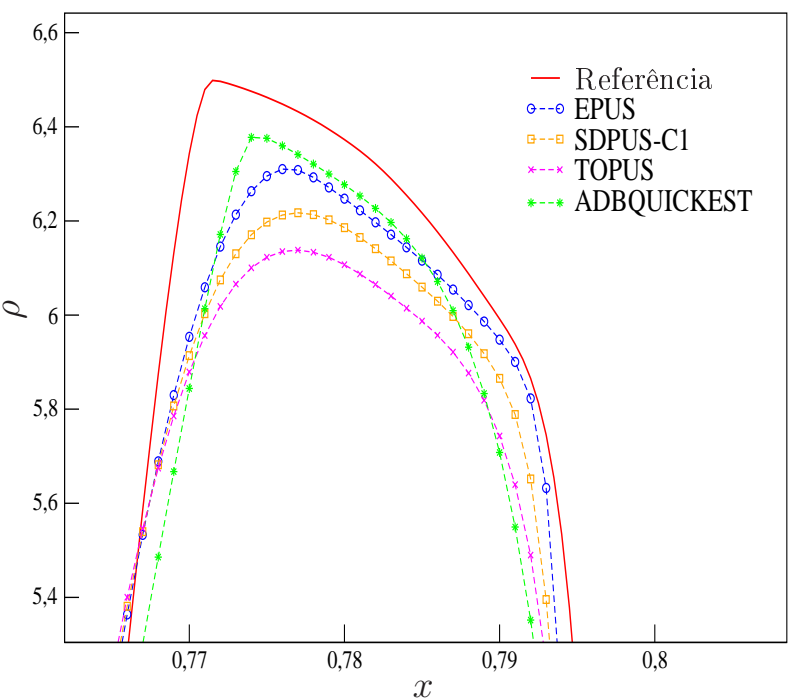

Figura 6.9: soluções de referência e numéricas para a densidade no problema Two Interacting Blast Waves. 


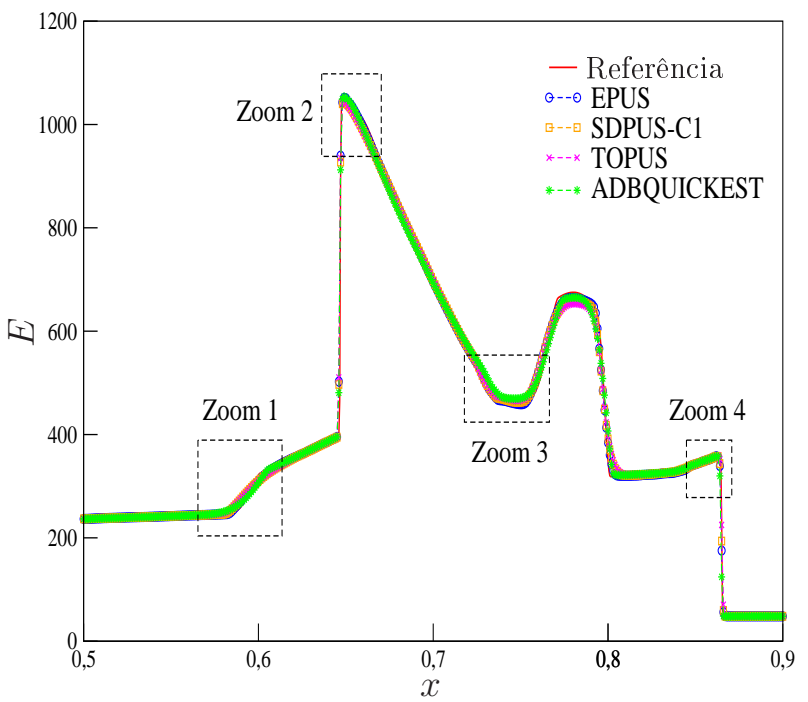

Zoom 1

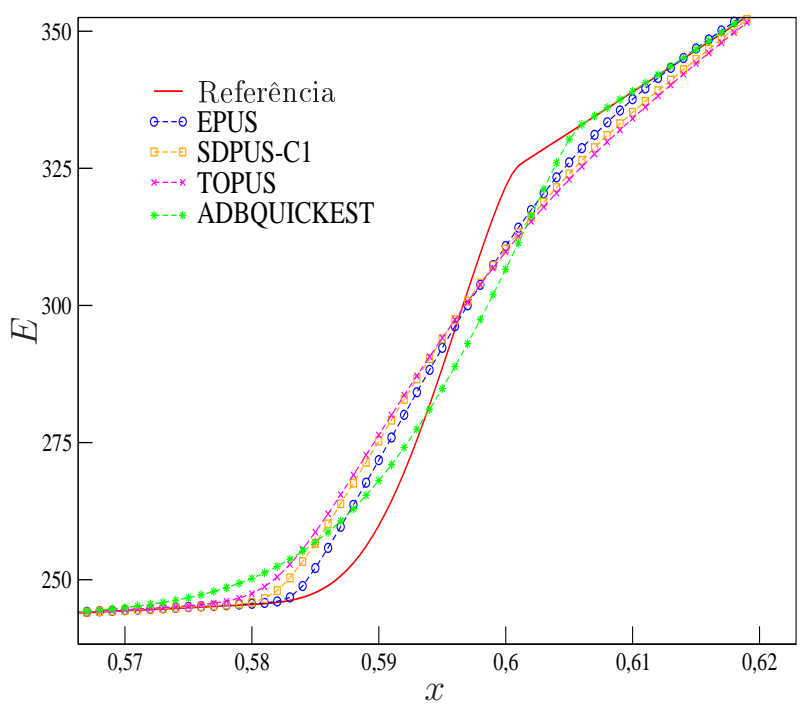

Zoom 3

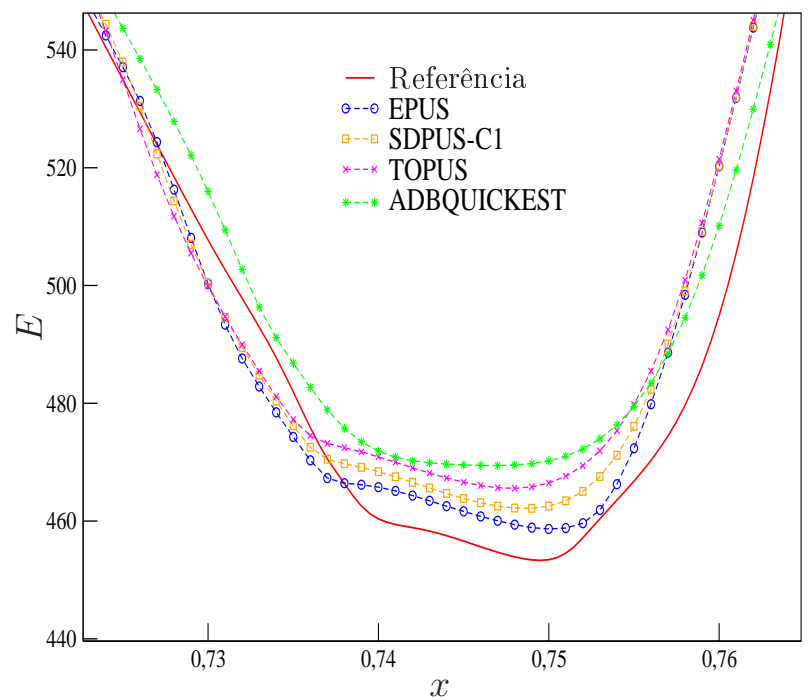

Zoom 2

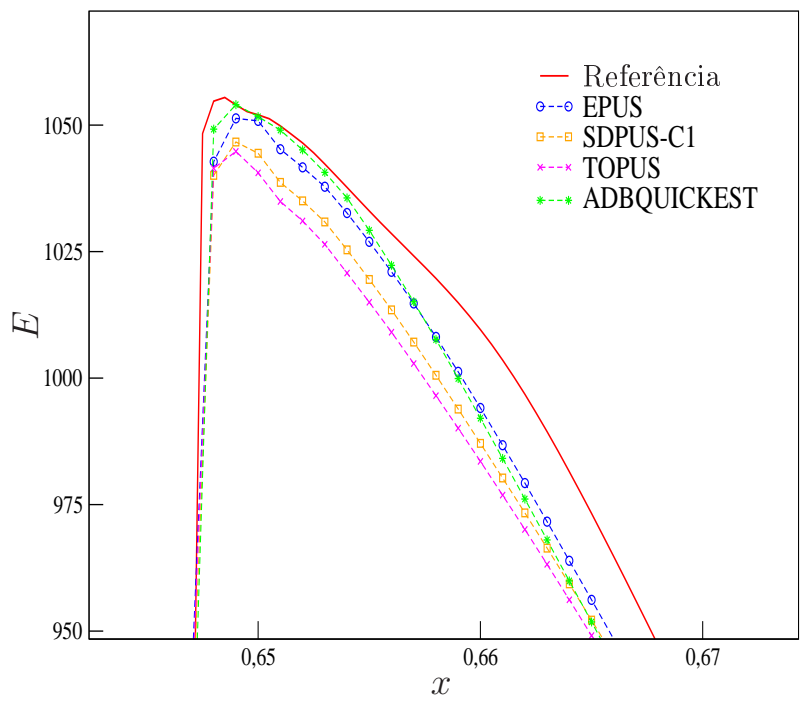

Zoom 4

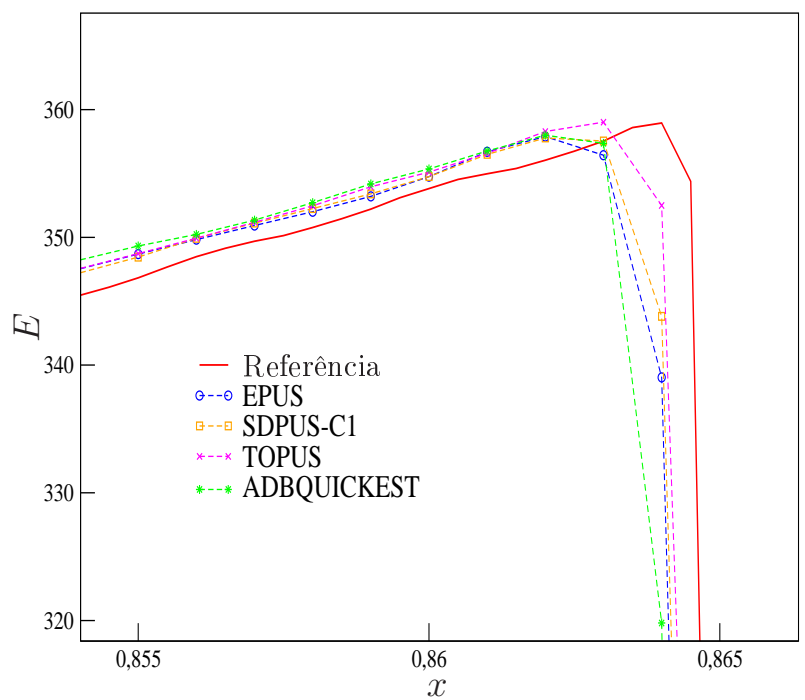

Figura 6.10: soluções de referência e numéricas para a energia total no problema Two Interacting Blast Waves. 


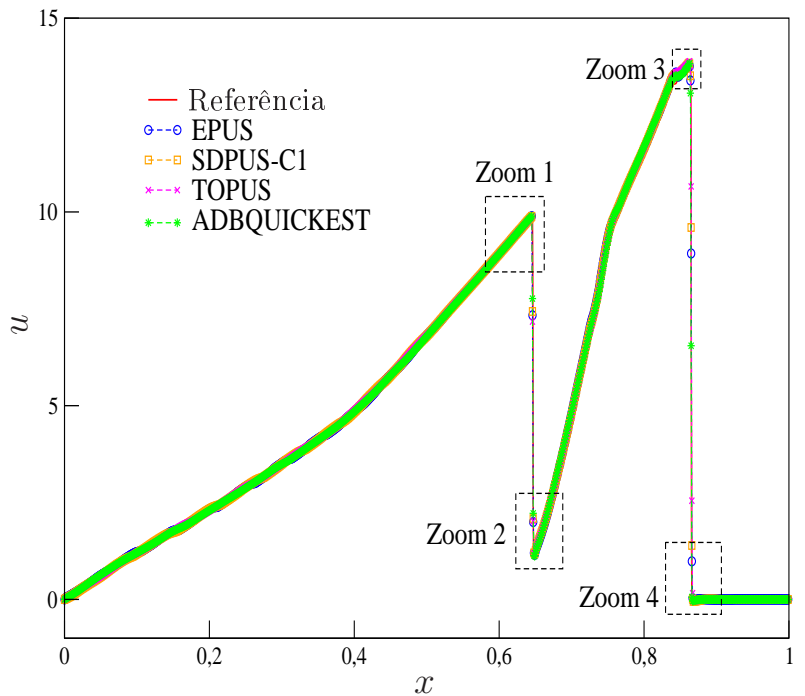

\section{Zoom 1}

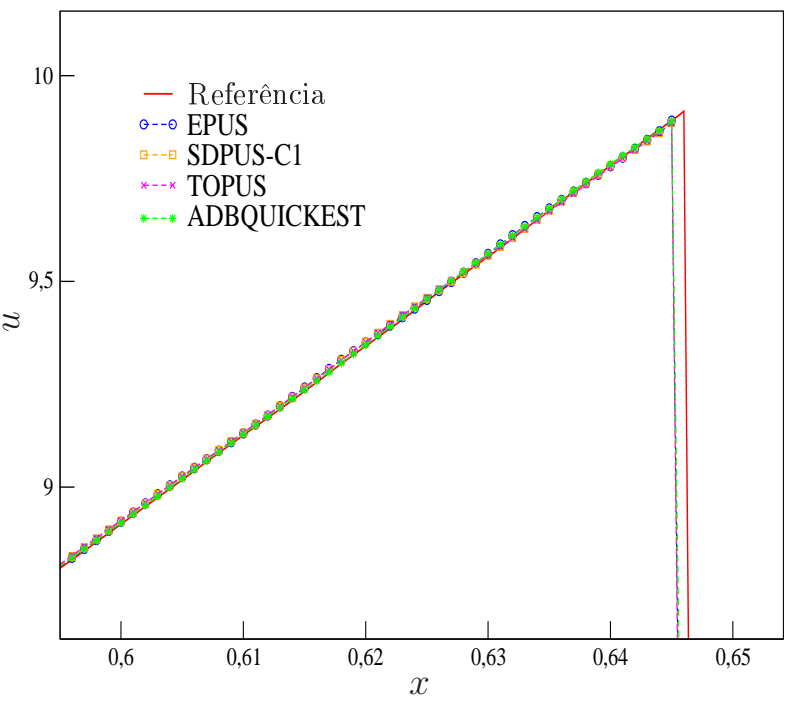

Zoom 3

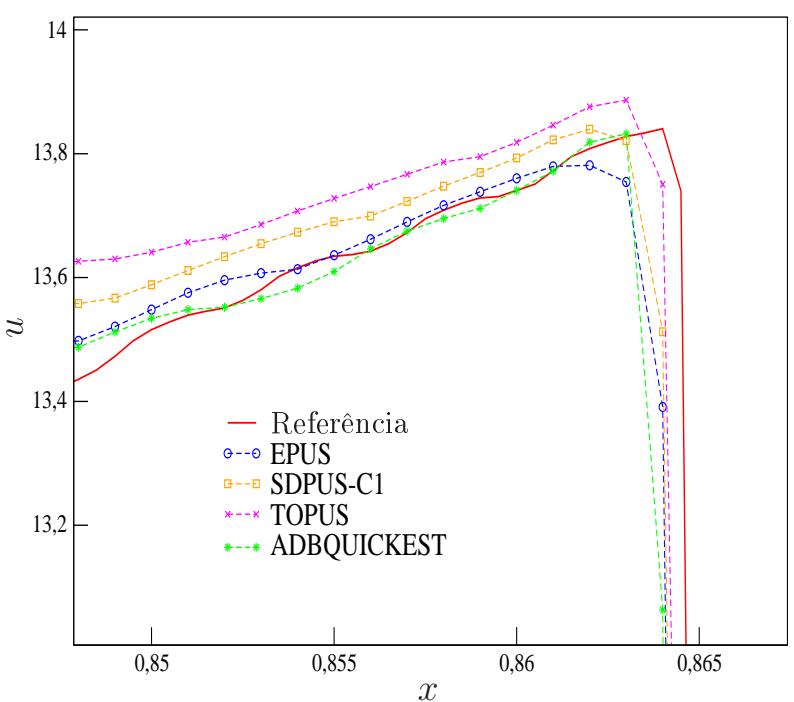

Zoom 2

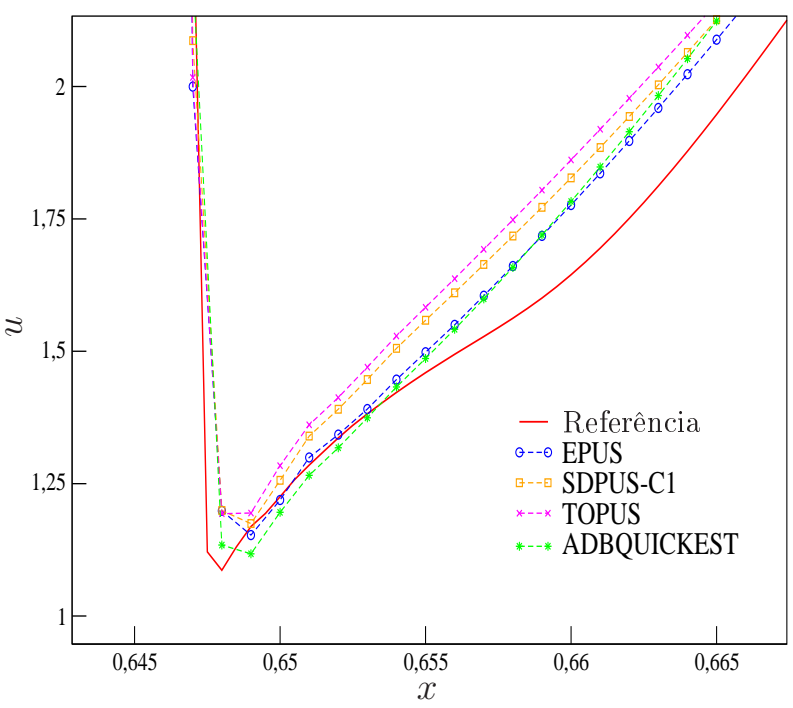

Zoom 4

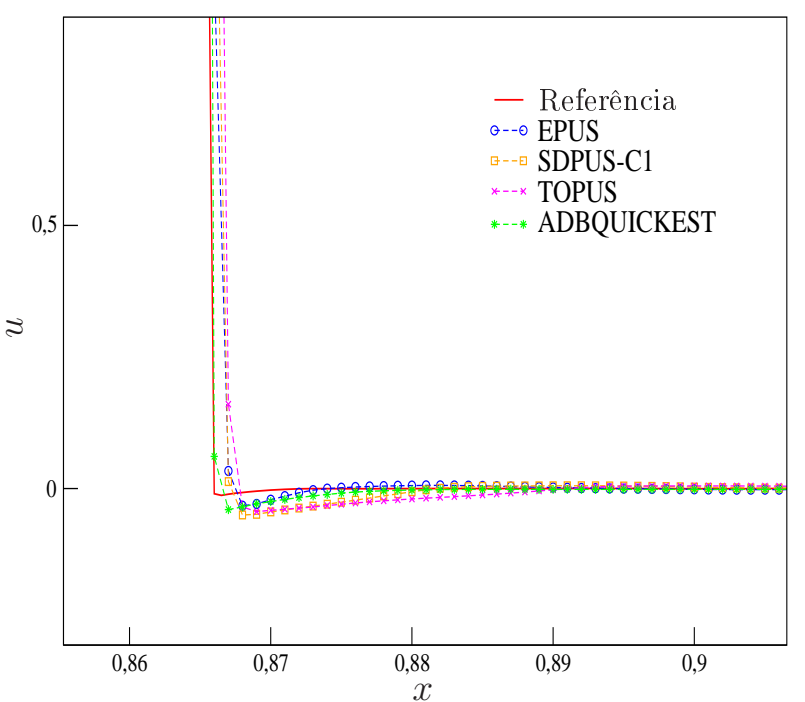

Figura 6.11: soluções de referência e numéricas para a velocidade no problema Two Interacting Blast Waves. 


\begin{tabular}{l|rccc}
\hline \hline Esquema & $N$ & $\left\|E_{h}\right\|_{1}$ & $\tilde{p}$ & Tempo de CPU \\
\hline EPUS & 125 & 0.186900 & - & 0.044 \\
& 250 & 0.098291 & 0.927138 & 0.108 \\
& 500 & 0.041372 & 1.248393 & 0.395 \\
& 1000 & 0.012660 & 1.708353 & 1.360 \\
\hline SDPUS & 125 & 0.202030 & - & 0.058 \\
& 250 & 0.113256 & 0.834988 & 0.112 \\
& 500 & 0.052581 & 1.106972 & 0.406 \\
& 1000 & 0.019254 & 1.449361 & 1.322 \\
\hline TOPUS & 125 & 0.219149 & - & 0.044 \\
& 250 & 0.125732 & 0.801561 & 0.104 \\
& 500 & 0.061682 & 1.027422 & 0.381 \\
& 1000 & 0.024237 & 1.347674 & 1.271 \\
\hline ADBQUICKEST & 125 & 0.202354 & - & 0.048 \\
& 250 & 0.114087 & 0.826747 & 0.111 \\
& 500 & 0.054632 & 1.062314 & 0.402 \\
& 1000 & 0.022263 & 1.295119 & 1.273 \\
\hline WACEB & 125 & 0.185689 & - & 0.033 \\
& 250 & 0.104136 & 0.834427 & 0.117 \\
& 500 & 0.046806 & 1.153707 & 0.367 \\
& 1000 & 0.016414 & 1.511779 & 1.239 \\
\hline \hline
\end{tabular}

Tabela 6.8: resultados obtidos para o Teste-8: erro relativo $E_{h}$ e estimativa para a ordem de convergência $\tilde{p}$, ambos obtidos na norma $L_{1}$, e tempo de CPU em cada malha.

Teste-9: neste teste apresenta-se o problema conhecido por tubo de choque de Sod [51]. Na Figura 6.12 é ilustrado as condições iniciais para um tubo de choque deste tipo, em que a Região 1 está à esquerda do choque e a Região 2 à direita dele. Nota-se que devem ser satisfeitas as seguintes condições: $p_{l}>p_{r}, \rho_{l}>\rho_{r}$ e $u_{l}=u_{r}=0$ (o fluido está inicialmente em repouso).

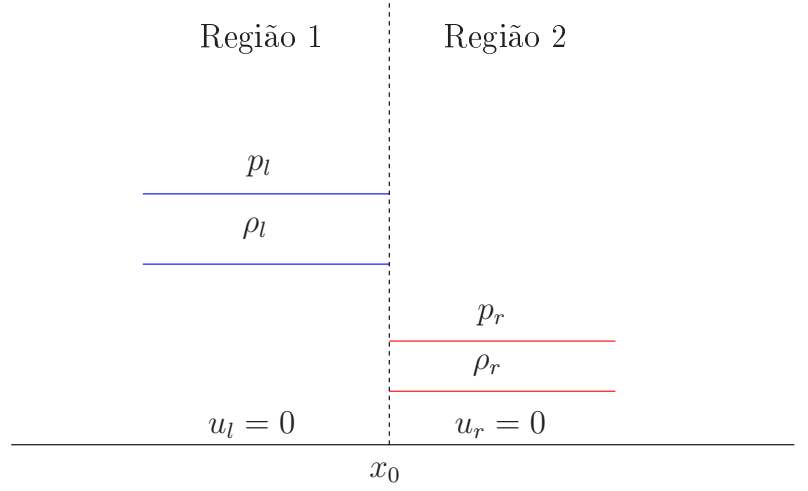

Figura 6.12: modelo do tubo de choque de Sod em $t=0$.

Neste teste, as condições iniciais para o tubo de choque de Sod são dadas por

$$
\left(\rho_{0}, u_{0}, p_{0}\right)^{T}=\left\{\begin{array}{l}
(1,0,1)^{T}, \quad x \leq 0.5 \\
(0.125,0,0.1)^{T}, \quad x>0.5 .
\end{array}\right.
$$


Para esta simulação, considera-se uma malha com 500 células computacionais, número de Courant $\theta=0.9$, tempo final de simulação $t=0.1$ e domínio computacional $x \in[0,1]$ $(\delta x=0.002)$. Para a solução de referência, utiliza-se o limitador MC aplicado a uma malha com 2000 células computacionais e número de Courant $\theta=0.1$. Os resultados obtidos seguem apresentados nas Figuras 6.13 e 6.14, em que são apresentados as soluções para densidade e energia interna, respectivamente. A partir destas figuras, é possível concluir que o esquema EPUS tem bom desempenho ao simular este problema de tubo de choque (tanto para os resultados da densidade quanto para a energia interna), sendo, entre os esquemas polinomiais, o melhor deles. No entanto, em algumas regiões, o ADBQUICKEST mostrou capturar melhor a solução. 


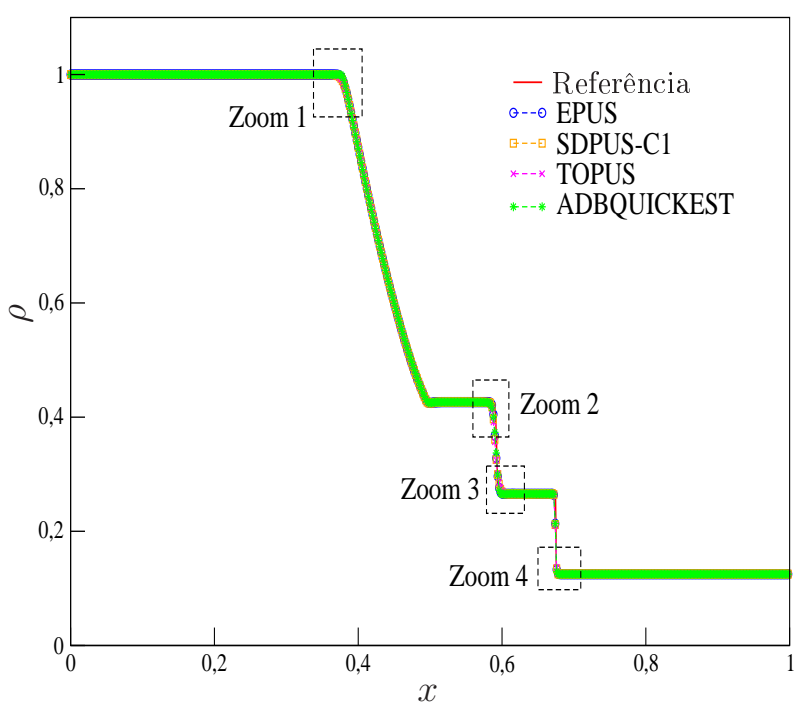

Zoom 1

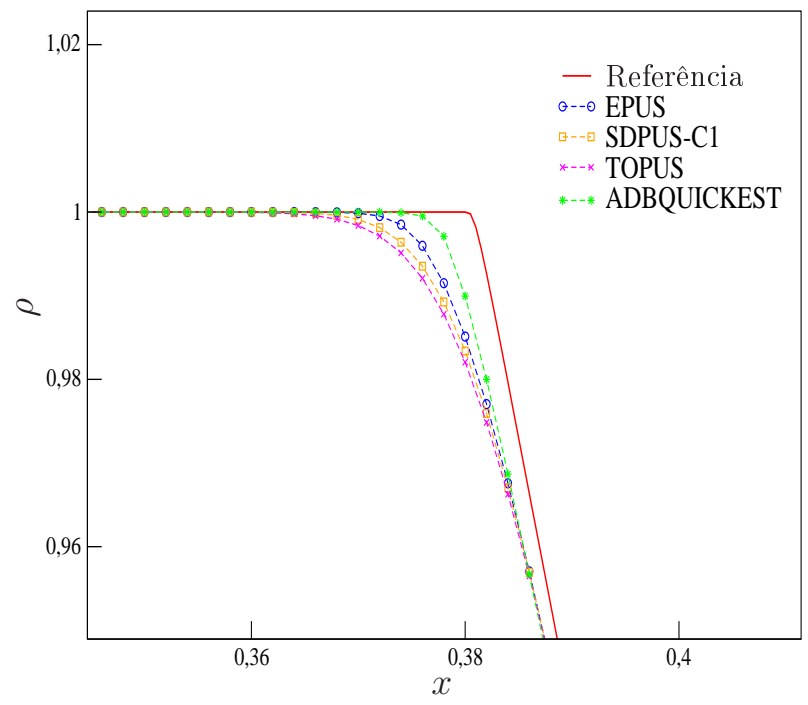

Zoom 3

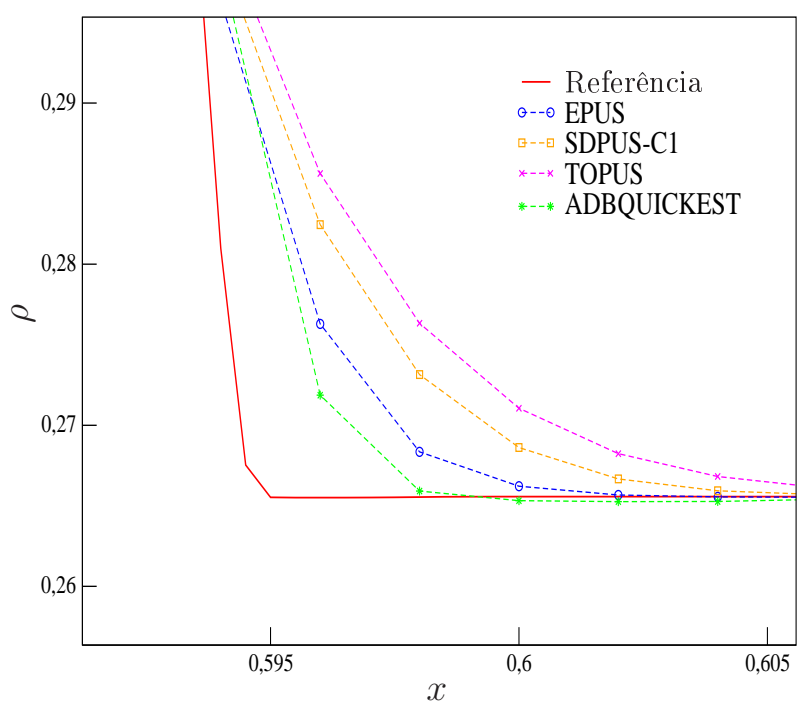

Zoom 2

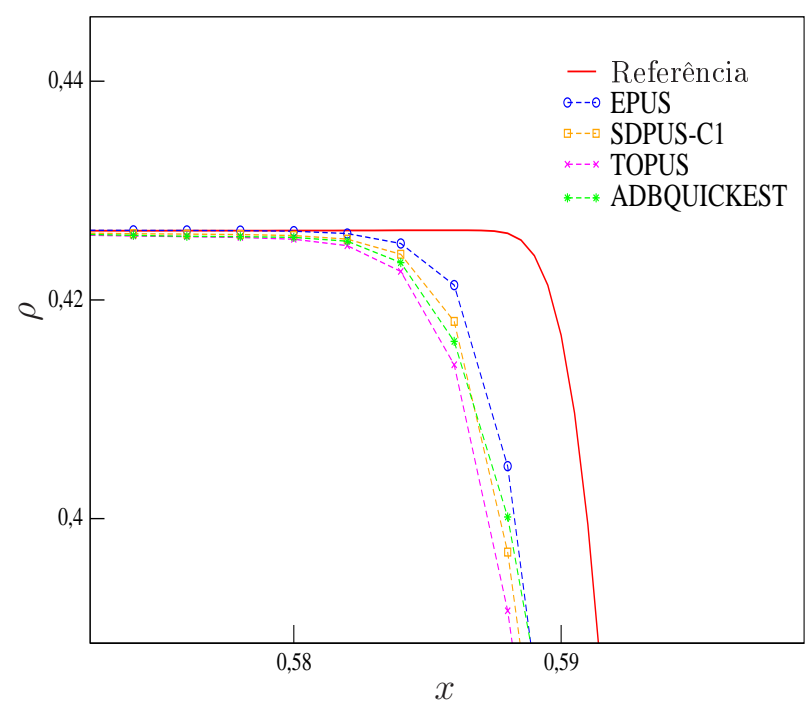

Zoom 4

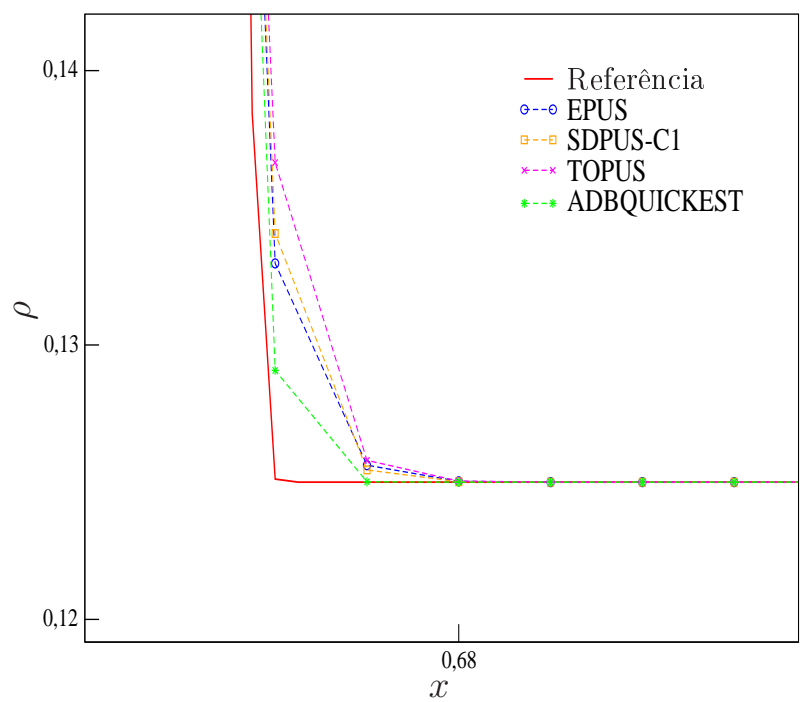

Figura 6.13: soluções de referência e numéricas para a densidade no problema do tubo de choque de Sod. 


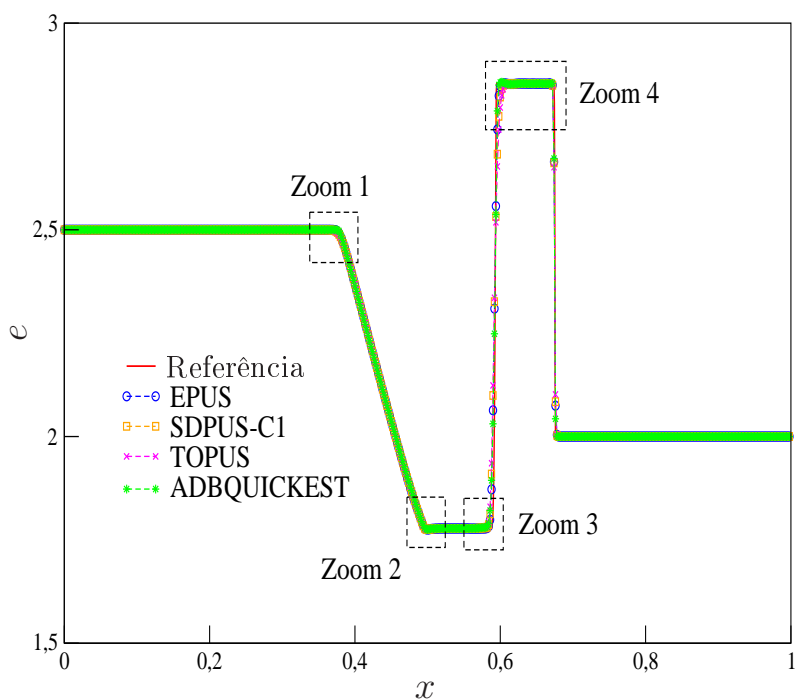

Zoom 1

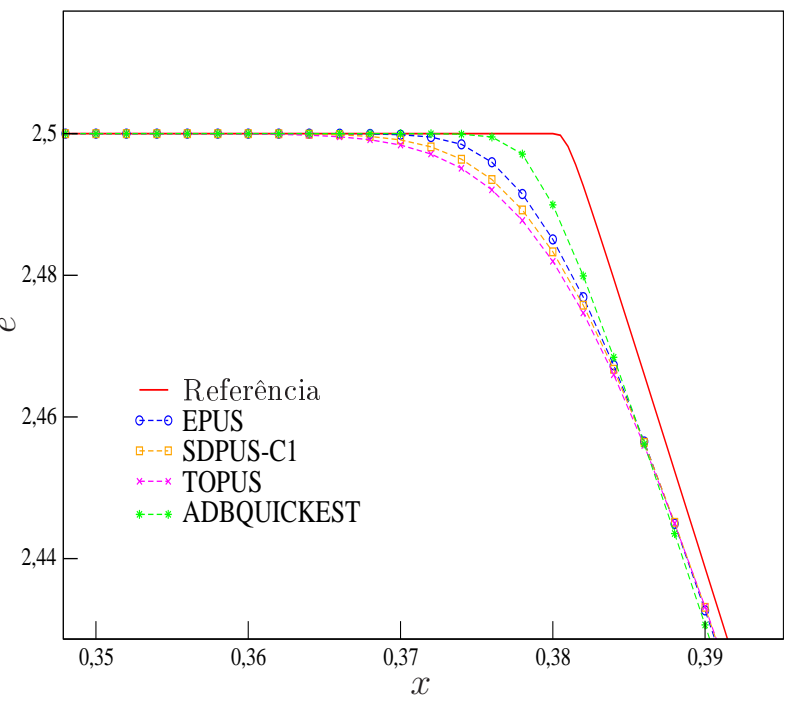

Zoom 3

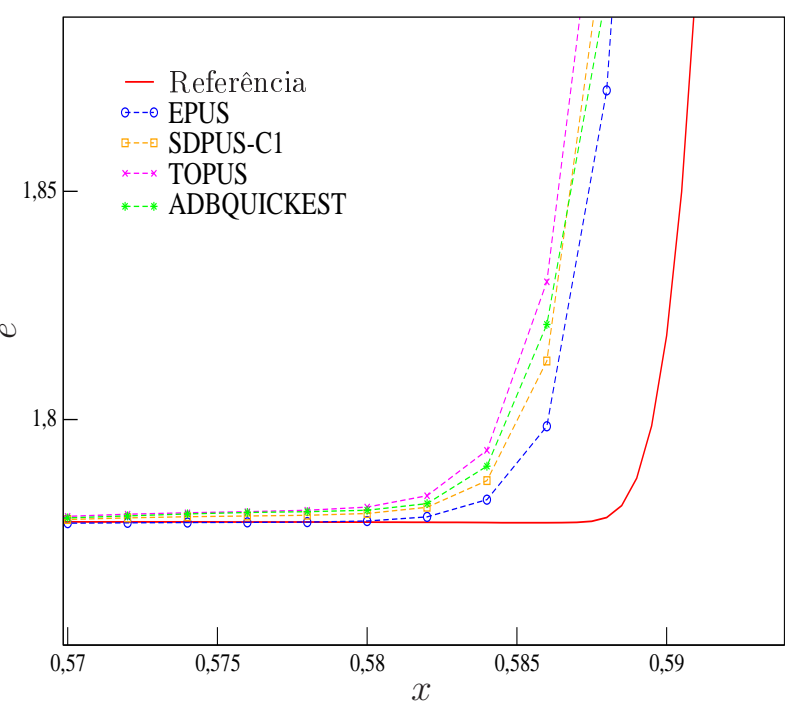

Zoom 2

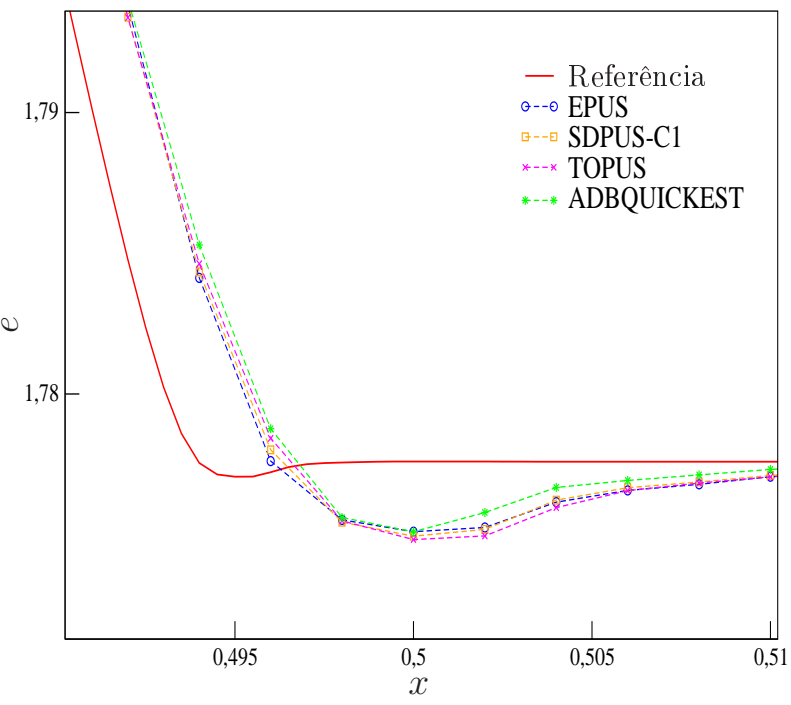

Zoom 4

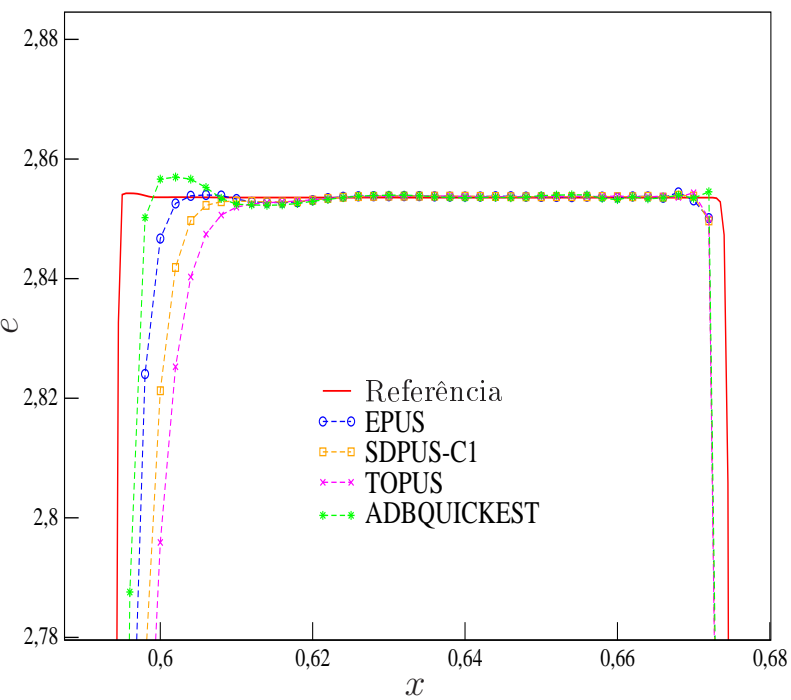

Figura 6.14: soluções de referência e numéricas para a energia interna no problema do tubo de choque de Sod. 


\section{Resultados Numéricos 2D e Axissimétricos}

O desempenho do esquema EPUS é verificado neste capítulo em leis de conservação 2D (Euler e águas rasas) e nas equações 2D e axissimétricas de Navier-Stokes. Para as leis de conservação, os resultados obtidos pelo esquema EPUS são confrontados com soluções de referência e também com os resultados obtidos por outros esquemas de alta resolução. Para os escoamentos incompressíveis modelados pelas equações de Navier-Stokes, os dados apresentados pelo esquema EPUS são comparados com resultados analíticos, experimentais, teóricos e numéricos presentes na literatura.

\subsection{Leis de Conservação 2D}

Nesta seção, apresentam-se os resultados numéricos obtidos pelo esquema EPUS para resolver dois sistemas hiperbólicos de leis de conservação 2D, a saber, Euler e águas rasas. Estes problemas foram simulados no ambiente computacional CLAWPACK ${ }^{1}$.

\subsubsection{Sistema Não-Linear de Euler}

O sistema hiperbólico não linear de Euler 2D, descrito na Seção 2.2.1, é utilizado aqui para modelar dois problemas da dinâmica dos gases, a saber, Four-shocks e Four-contacts, os quais são apresentados a seguir.

Teste-1: neste teste resolve-se o fenômeno conhecido por Four-shocks problem (ver [9, 32]), que consiste num problema de Riemann 2D com estados iniciais constantes dados em quatro quadrantes, definidos no domínio $[0,1] \times[0,1]$ e com um ponto de contato localizado em $(0.8,0.8)$.

\footnotetext{
${ }^{1}$ http://www.amath.washington.edu/ claw/
} 
Este problema é caracterizado pela interação de quatro ondas de choque. Seus estados iniciais são dados por

$$
\left[\rho_{0}, u_{0}, v_{0}, p_{0}\right]^{T}= \begin{cases}{\left[\begin{array}{l}
1.5,0,0,1.5 \\
{[}
\end{array}\right]^{T},} & {[0.8,1] \times[0.8,1]} \\
{[0.13799,1.2060454,1.2060454,0.0290323]^{T},} & {[0,0.8] \times[0,0.8],} \\
{[0.5322581,1.2060454,0,0.3]^{T},} & {[0,0.8] \times[0.8,1]} \\
{[0.5322581,0,1.2060454,0.3]^{T},} & {[0.8,1] \times[0,0.8]}\end{cases}
$$

Para a simulação deste teste considera-se uma malha com $200 \times 200$ células computacionais $(\delta x=\delta y=0.005)$, número de Courant $\theta=0.8$ e tempo final de simulação $t f=0.8$. Os resultados obtidos para o perfil da densidade $\rho$ no plano $x \perp y$ são mostrados na Figura 7.1, em que é possível observar que todos os esquemas apresentados conseguiram simular satisfatoriamente o fenômeno proposto, sendo que os melhores resultados foram obtidos pelo esquema EPUS, o qual captura melhor a solução.

A partir de uma melhor análise dos resultados obtidos neste teste, a qual é apresentada na Figura 7.2, avalia-se os valores de $\rho$ sobre a reta $y=x$, plotando-os em relação a $x$. Analisando esta figura, conclui-se que, de fato, o esquema que melhor resolve este problema é o EPUS, capturando com bom desempenho as regiões de picos e vales, sem suavizá-las, comportamento este muito evidenciado pelo esquema ADBQUICKEST.

Ainda, com o objetivo de fazer uma análise do ponto de vista quantitativo, calcula-se também a ordem de convergência observada. Os resultados obtidos seguem exibidos na Tabela 7.1. De um modo geral, todos os esquemas obtiveram resultados semelhantes, sendo que o esquema EPUS apresenta, em sua maioria, os melhores resultados.

Com o objetivo de fazer uma análise da solução com refino da malha, procurando evidenciar vantajosas propriedades do esquema EPUS, este mesmo problema é resolvido utilizando uma malha com $1000 \times 1000$ células computacionais (ver Cada [9]) $(\delta x=\delta y=0.001), \theta=0.8 \mathrm{e}$ $t f=0.8$. Os resultados obtidos para o perfil da densidade $\rho$ no plano $x \perp y$ são apresentados na Figura 7.3, em que pode-se observar que o esquema EPUS (com parâmetro livre $\lambda=95$ ) é o que menos amortece as estruturas vorticais formadas no problema, mostrando ser um esquema que introduz pouca dissipação numérica. Observe ainda, nesta mesma figura, mais um exemplo de outra propriedade do esquema EPUS já apresentada anteriormente: os melhores resultados são obtidos pelo novo esquema com parâmetro livre $\lambda=95$ em problemas com choques (que é o caso deste teste), enquanto $\operatorname{com} \lambda=16$ apresenta pior desempenho neste tipo de problema. 


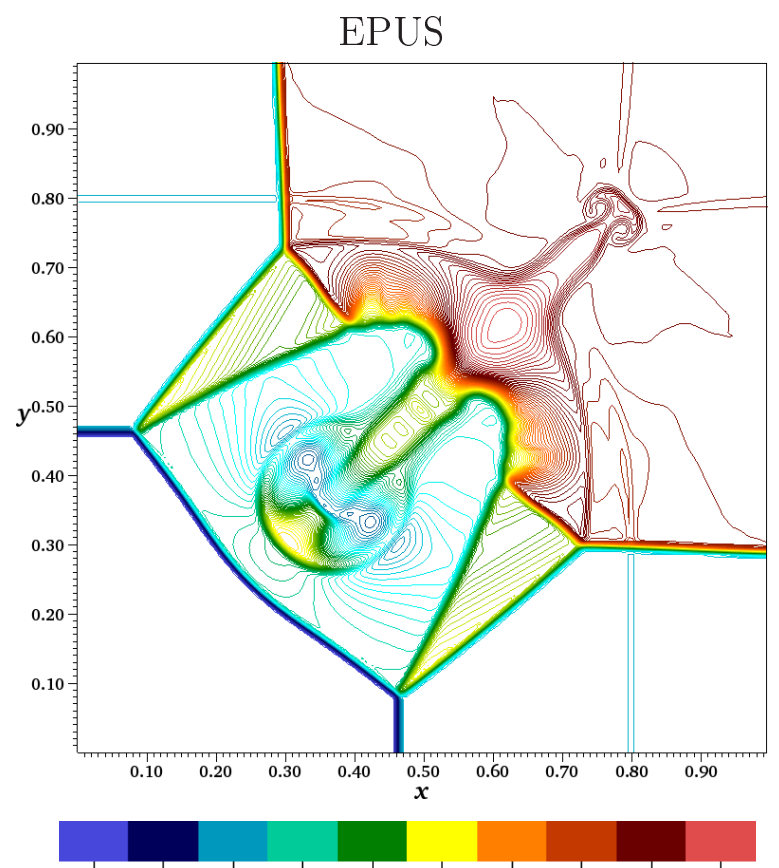

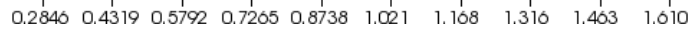

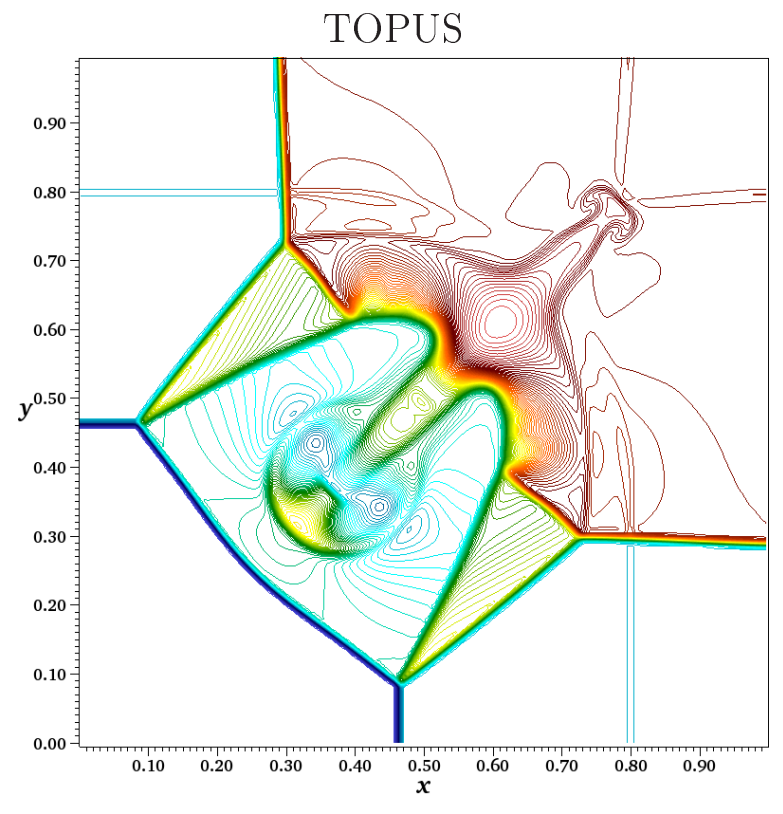

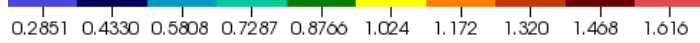
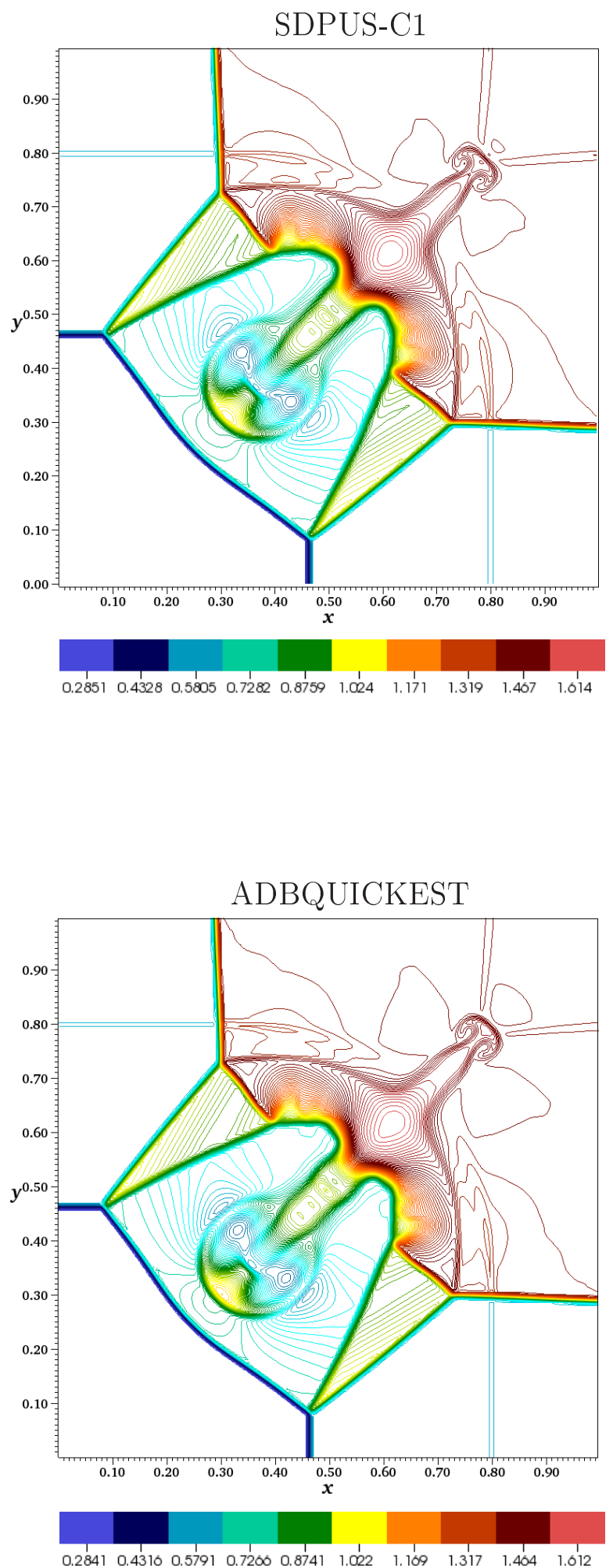

Figura 7.1: perfil da densidade para o problema Four-shocks problem (Teste-1). 

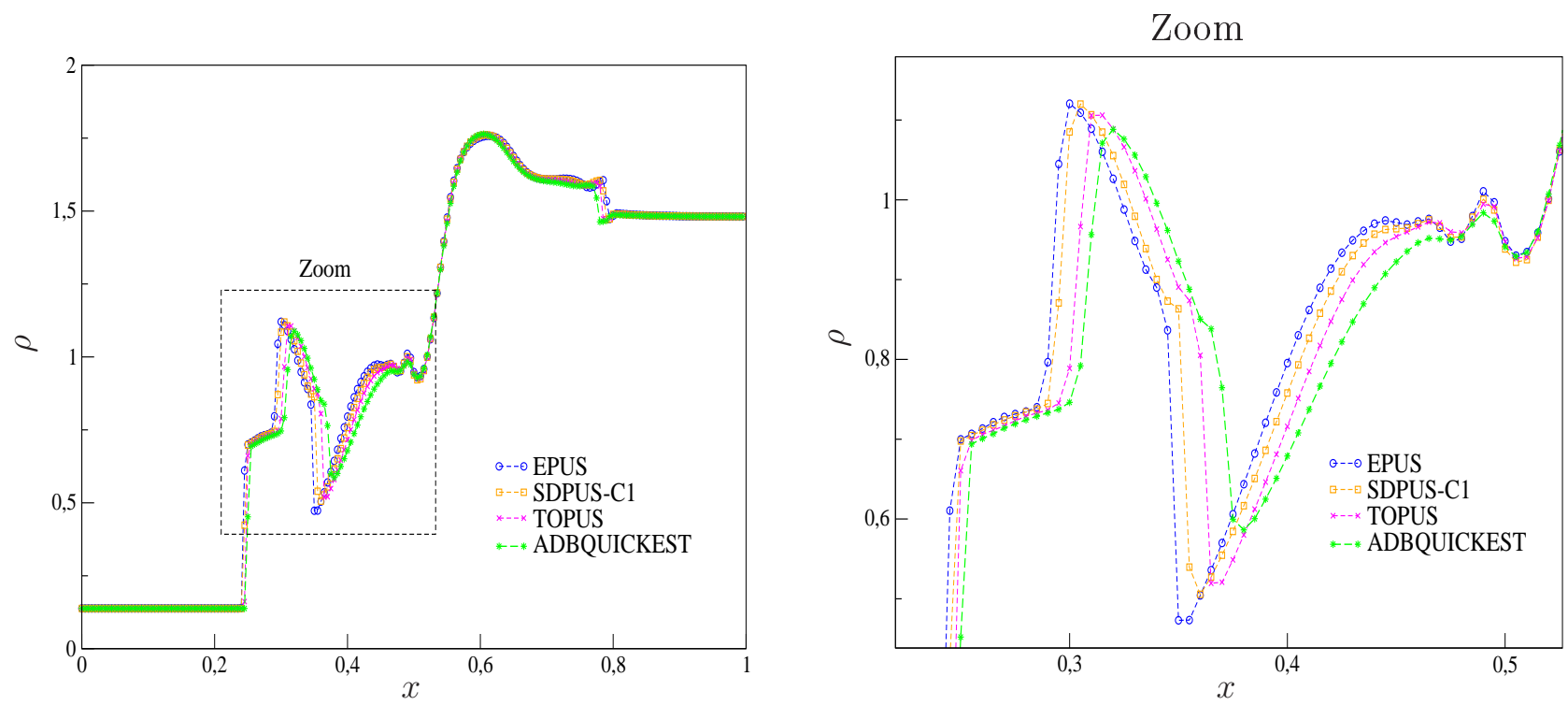

Figura 7.2: comportamento da densidade sobre a reta $y=x$ para o problema Four-shocks problem (Teste-1).

\begin{tabular}{l|r|c|c|c|c}
\hline \hline Esquemas & \multicolumn{1}{|c}{$N$} & $t f=0.2$ & $t f=0.4$ & $t f=0.6$ & $t f=0.8$ \\
\hline EPUS & 16 & - & - & - & - \\
& 32 & 2.802421 & 2.558469 & 1.989391 & 3.148710 \\
& 64 & 1.149728 & 1.468347 & 1.528174 & 0.655765 \\
& 128 & 1.452255 & 2.274759 & 1.606929 & 1.477884 \\
\hline SDPUS-C1 & 16 & - & - & - & - \\
& 32 & 2.786139 & 2.577962 & 1.983243 & 3.119330 \\
& 64 & 1.183948 & 1.446474 & 1.492269 & 0.675083 \\
& 128 & 1.433276 & 2.282586 & 1.614194 & 1.456111 \\
\hline TOPUS & 16 & - & - & - & - \\
& 32 & 2.781235 & 2.592425 & 1.979919 & 3.099280 \\
& 64 & 1.206041 & 1.432068 & 1.474187 & 0.688797 \\
& 128 & 1.417527 & 2.281726 & 1.612432 & 1.426924 \\
\hline ADBQUICKEST & 16 & - & - & - & - \\
& 32 & 2.766081 & 2.595229 & 1.983185 & 3.079302 \\
& 64 & 1.215765 & 1.402327 & 1.632650 & 0.701337 \\
& 128 & 1.418710 & 2.248497 & 1.564420 & 1.432594 \\
\hline \hline
\end{tabular}

Tabela 7.1: ordem de convergência $\tilde{p}$ na norma $L_{1}$ para o Teste-1 em diferentes tempos. 


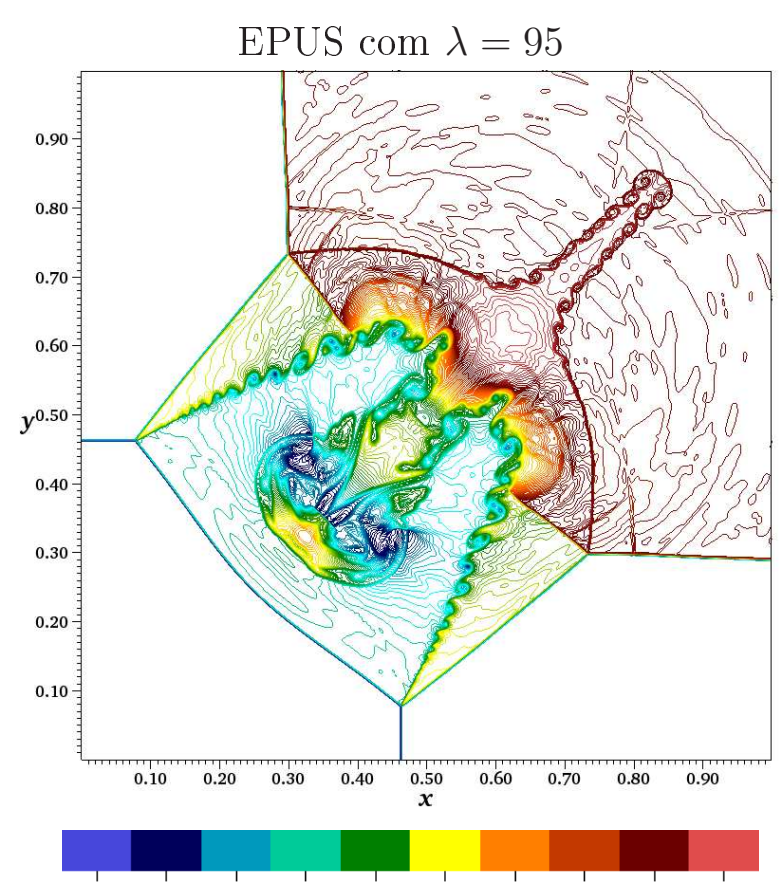

$\begin{array}{llllllllll}0.2826 & 0.4287 & 0.5747 & 0.7208 & 0.8008 & 1.013 & 1.159 & 1.305 & 1.451 & 1.597\end{array}$

\section{SDPUS-C1}

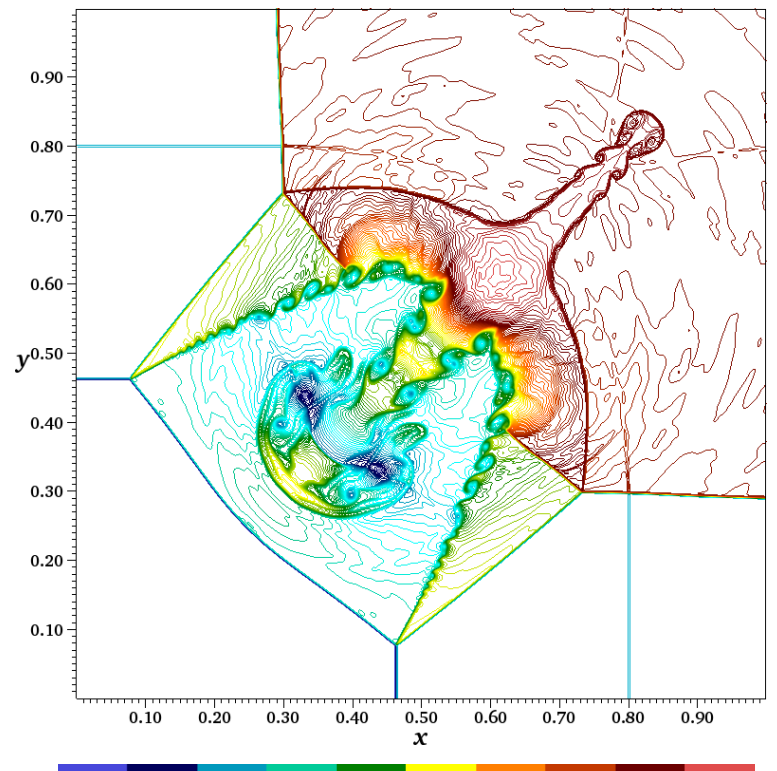

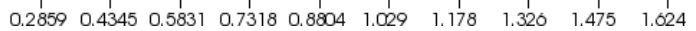

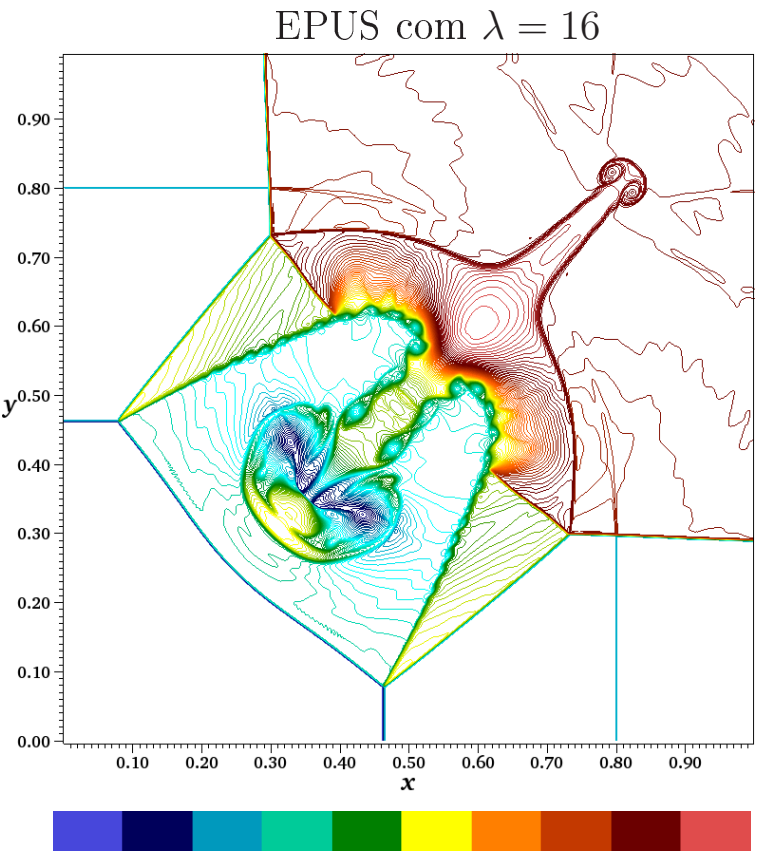

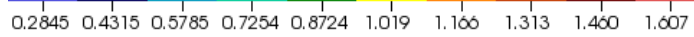

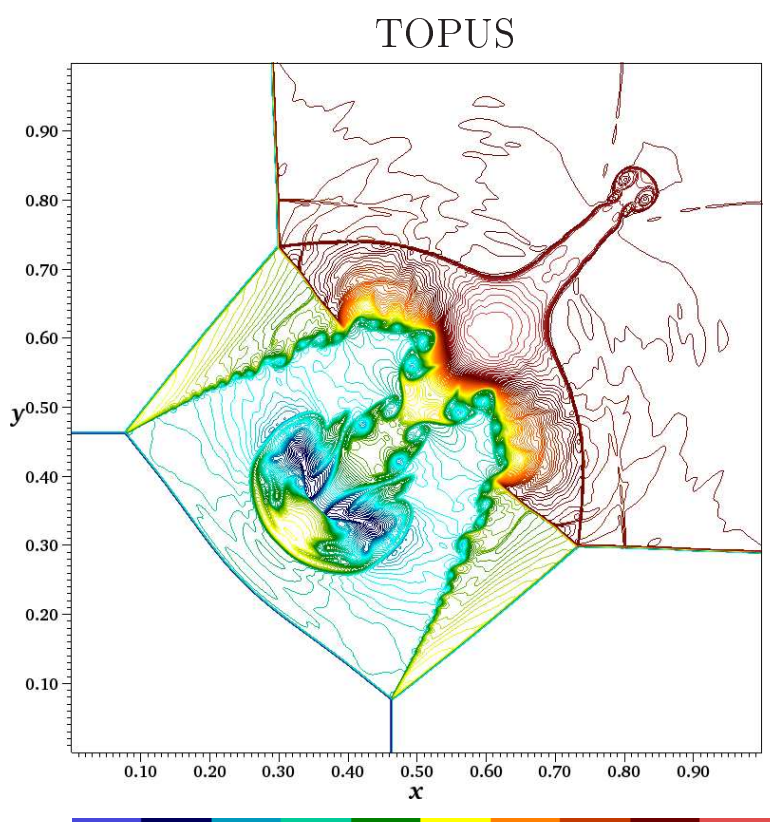

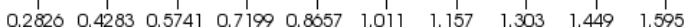

Figura 7.3: perfil da densidade numa malha refinada para o Four-shocks problem (Teste-1). 

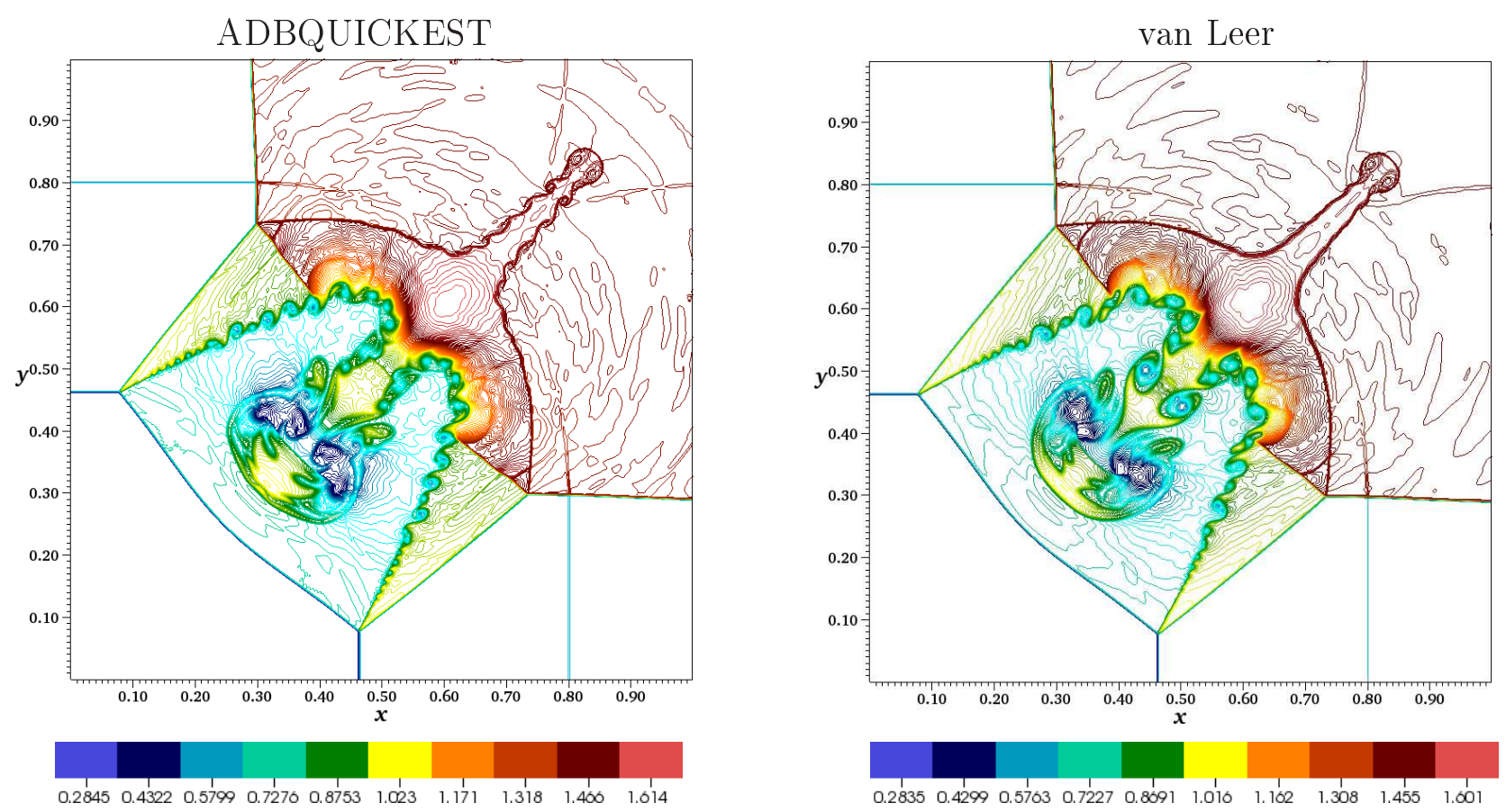

Figura 7.4: continuação da Figura 7.3.

Teste-2: este teste também consiste num problema de Riemann 2D, sendo conhecido por Four-contacts problem (ver [9]). A estrutura de sua solução é dada por um vórtice girando no sentido horário. Neste problema, duas ondas de choque são geradas através das linhas de contato, as quais se propagam para fora em forma de espiral. A condição inicial para este problema é:

$$
\left[\rho_{0}, u_{0}, v_{0}, p_{0}\right]^{T}= \begin{cases}{\left[\begin{array}{ll}
1,0.75,-0.5,1 \\
{[2,0.75,0.5,1}
\end{array}\right]^{T},} & {[0.5,1] \times[0.5,1]} \\
{\left[\begin{array}{ll}
1,-0.75,0.5,1 \\
{[3,}
\end{array}\right]^{T},} & {[0,0.5) \times[0.5,1]} \\
{[3,-0.75,-0.5,1]^{T},} & {[0.5,1] \times[0,0.5)}\end{cases}
$$

Nesta simulação, considera-se uma malha com $1500 \times 1500$ células computacionais, número de Courant $\theta=0.8$, tempo final de simulação $t f=0.8$ e domínio $[0,1] \times[0,1](\delta x=\delta y=$ 0.00067). Os resultados obtidos para o perfil da densidade $\rho$ seguem na Figura 7.5, onde pode-se observar que, mais uma vez, o esquema EPUS é o que menos amortece as estruturas vorticais que surgem neste fenômeno, mostrando ser, dentre os esquemas testados, o que introduz menor dissipação numérica. 


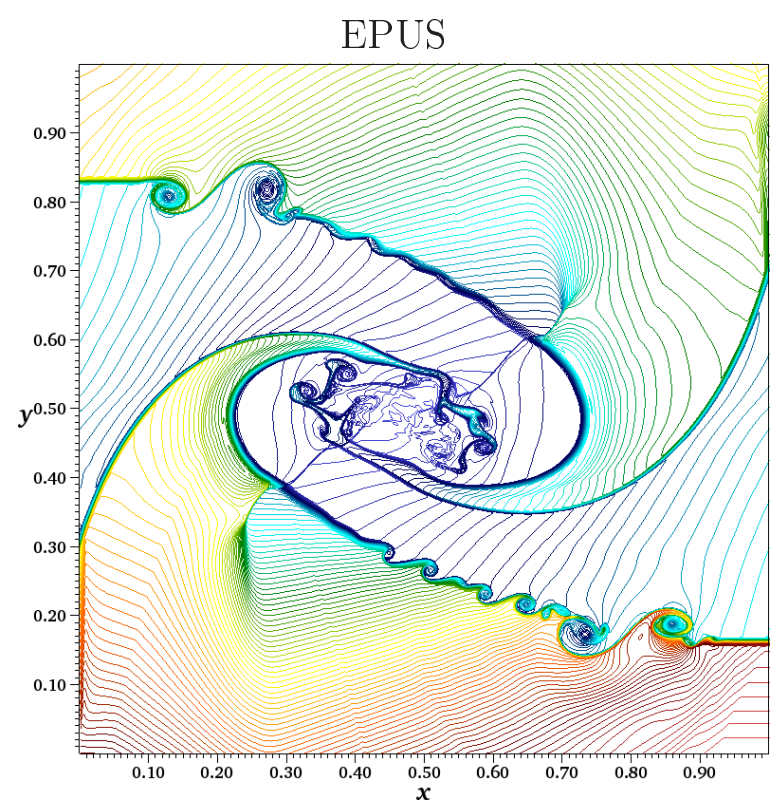

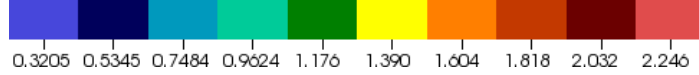

TOPUS

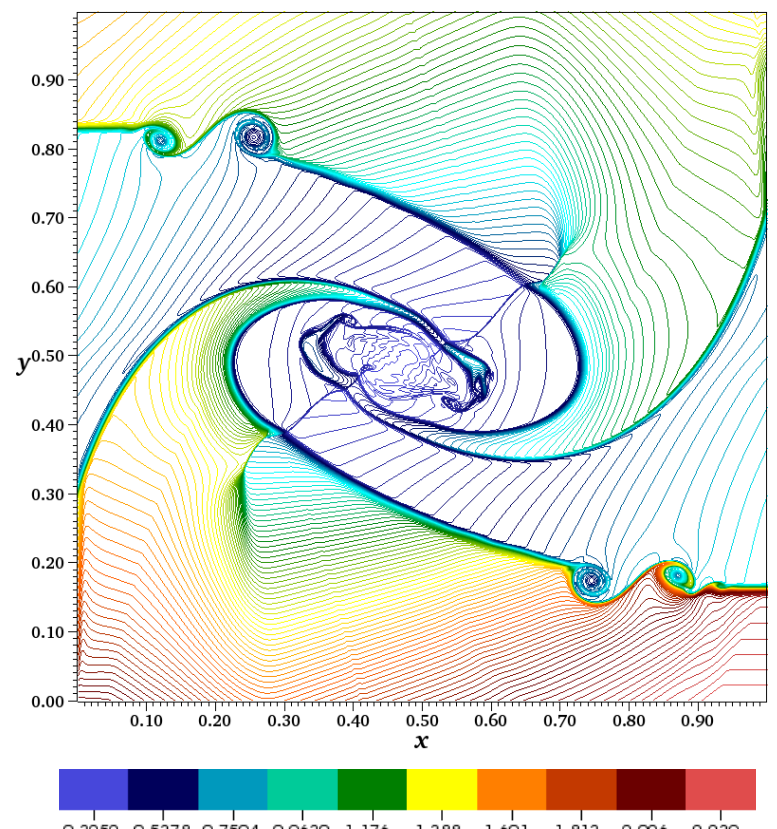

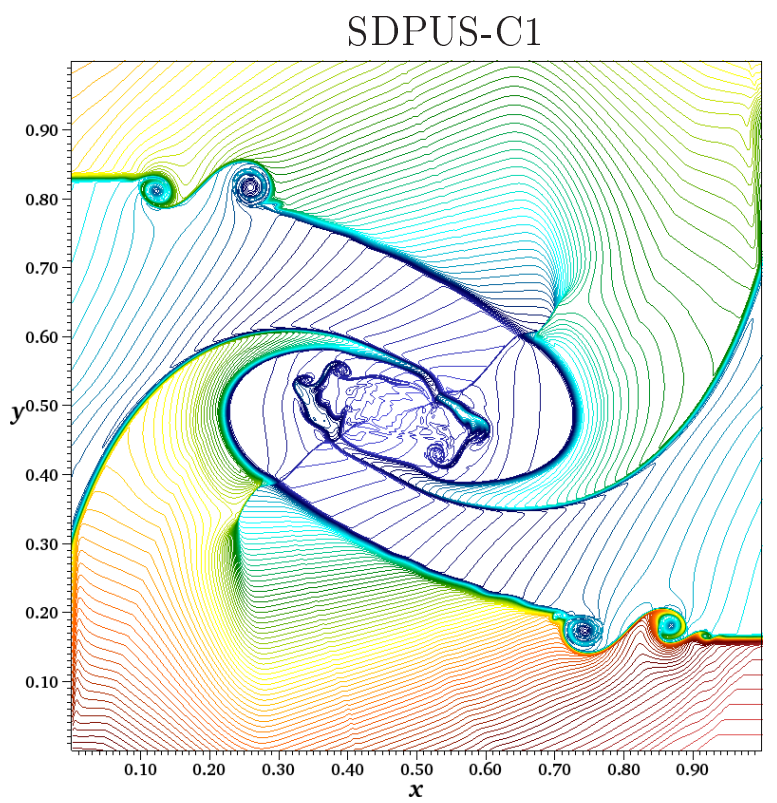

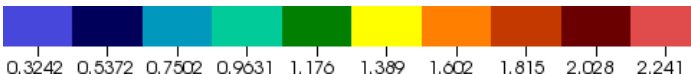

ADBQUICKEST

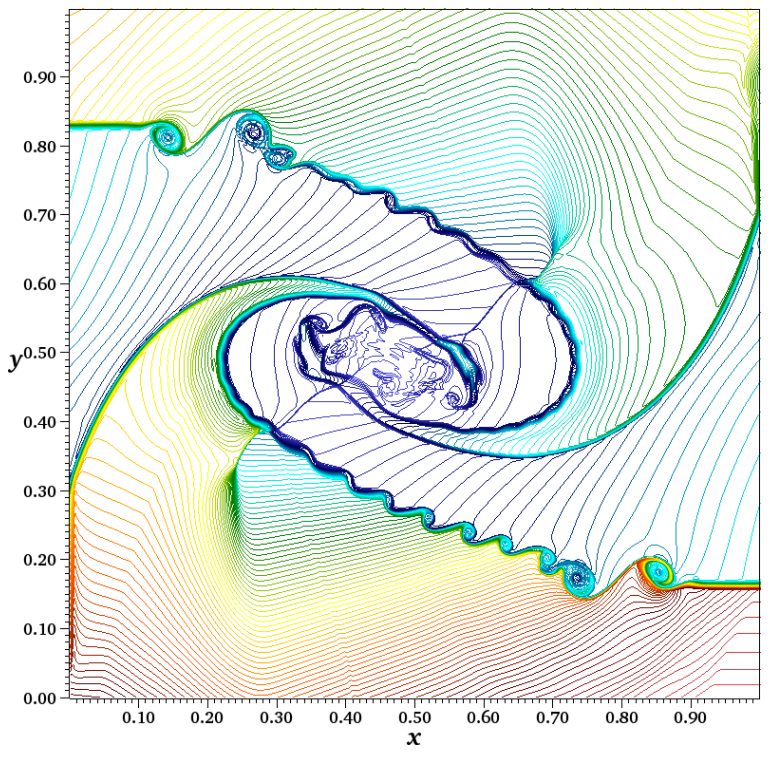

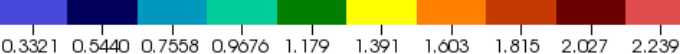

Figura 7.5: perfil da densidade numa malha refinada para o Four-contacts problem (Teste-2).

\subsubsection{Sistema Não-Linear de Águas Rasas}

O sistema hiperbólico não linear de águas rasas foi apresentado na Seção 2.2.2. Neste trabalho, estas equações modelam o problema conhecido como radial dam-break (quebra de barreira radial) [32], o qual é descrito e resolvido numericamente a seguir. 
Teste-3: resolve-se aqui o problema conhecido por radial dam-break problem (ver, por exemplo, [32]), que consiste numa porção de fluido circular inicialmente em repouso confinado por uma barreira (ver Figura 7.6 (a)). Esta barreira é removida instantaneamente, formando uma onda de choque que se espalha radialmente para fora, enquanto uma onda de rarefação se propaga para o interior (ver Figura 7.6 (b)). Inicialmente, a altura do fluido dentro da barreira é $h=2$ e $h=1$ fora dela. De acordo com LeVeque [32], este problema é similar a estrutura do problema de Riemann 1D de quebra de barreira. Levando em conta essa afirmação, considera-se a solução deste problema 1D como solução de referência para este teste. Esta solução é calculada resolvendo o seguinte sistema 1D de águas rasas com termo fonte:

$$
\begin{aligned}
h_{t}+(h U)_{r} & =-\frac{h U}{r} \\
(h U)_{t}+\left(h U^{2}+\frac{1}{2} g h^{2}\right)_{r} & =-\frac{h U^{2}}{r}
\end{aligned}
$$

onde $U(r, t)$ é a velocidade radial e $h$ é a altura como função de $r$ (distância a partir da origem).

(a) $t=0$

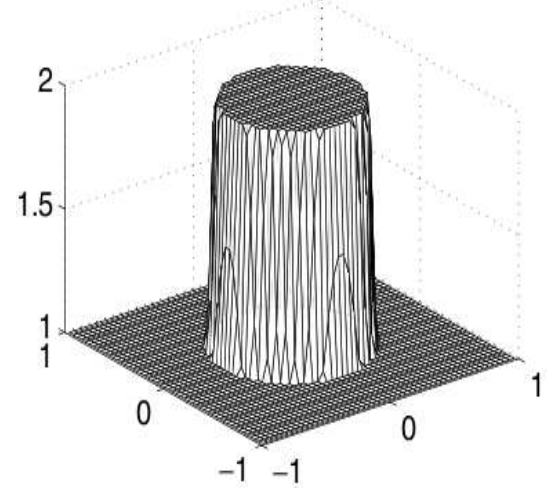

(b) $t=0.25$

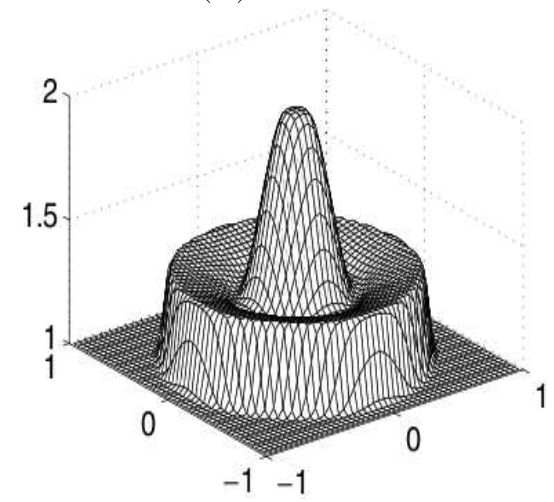

Figura 7.6: radial dam-break problem: comportamento da altura da porção de fluido nos instantes (a) $t=0$ e (b) $t=0.25$ (figura extraída de LeVeque [31]).

Para a simulação deste problema, considera-se, para as soluções numéricas, uma malha com $125 \times 125$ células computacionais, número de Courant $\theta=0.9$, tempo final de simulação $t f=1.5$ e domínio computacional $[-2.5,2.5] \times[-2.5,2.5](\delta x=\delta y=0.04)$. Para o cálculo da solução de referência, utiliza-se o limitador de fluxo MC e uma malha com $N=2000$ células computacionais.

Na Figura 7.7 apresenta-se o perfil da altura $h$ no plano $x \perp y$, em que é possível concluir que todos os esquemas capturam satisfatoriamente o fenômeno. No entanto, para uma melhor análise dos resultados obtidos, nas Figuras 7.8 e 7.9 plota-se os valores de $h$ em função de $x$ sobre a reta $y=0$. Nestas figuras, as soluções numéricas são comparadas com a solução de 
referência sugerida por LeVeque [32] (ver Eq. 7.3). Analisando-as é possível concluir que o esquema EPUS é o que captura melhor o fenômeno na região apresentada no Zoom 1 (região com descontinuidade e rarefação), enquanto que o esquema ADBQUICKEST é o que apresenta melhor desempenho em Zoom 2 (região com uma onda de choque).
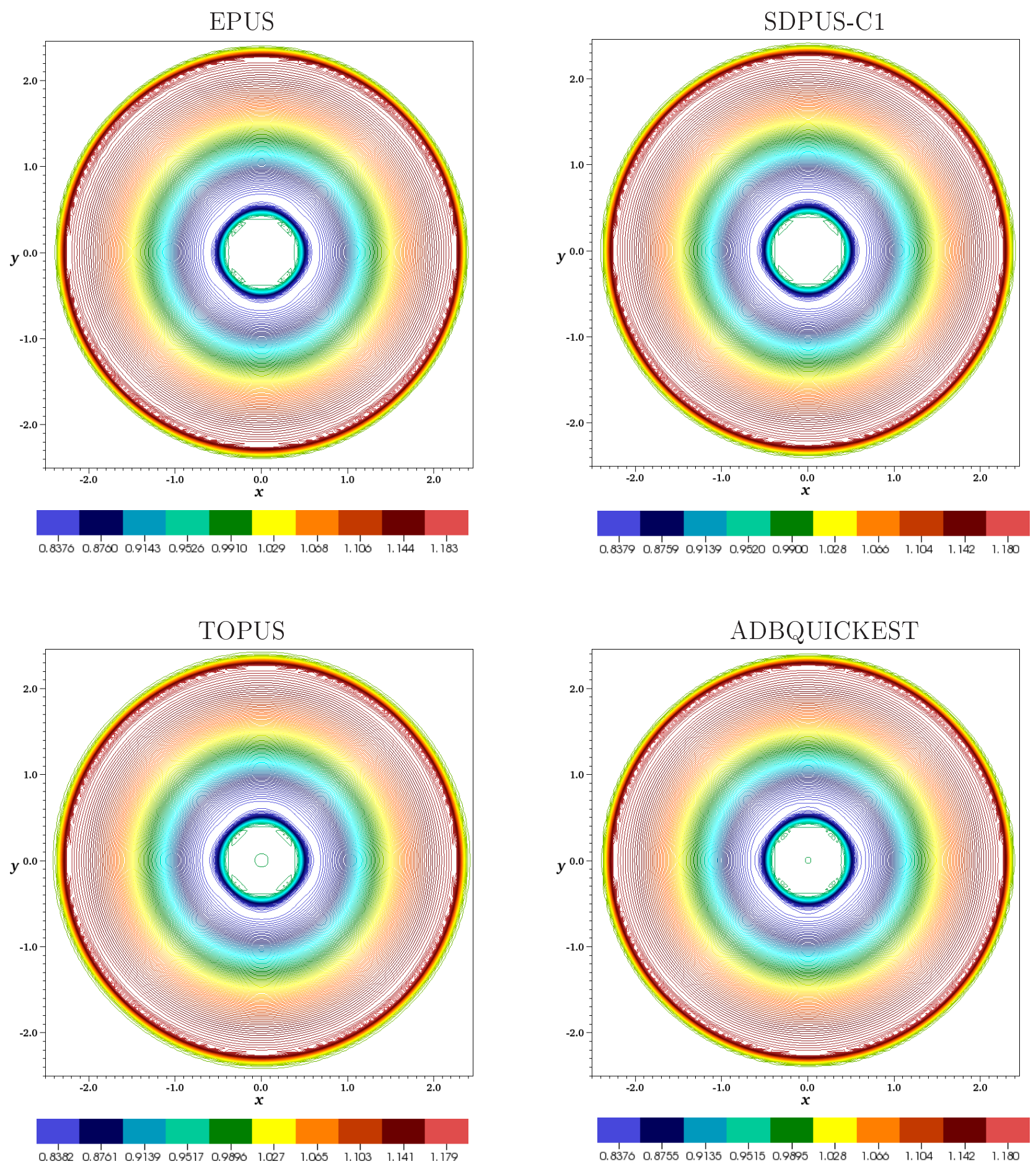

Figura 7.7: perfil da altura para o problema radial dam-break problem (Teste-3). 


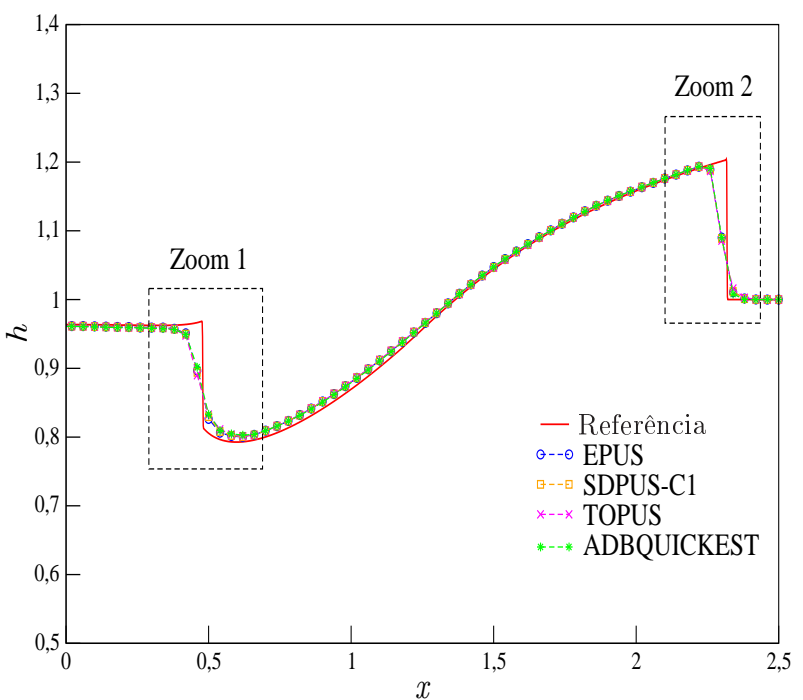

Figura 7.8: comportamento da altura sobre a reta $y=0$ para o problema radial dam-break problem (ver Teste-3).
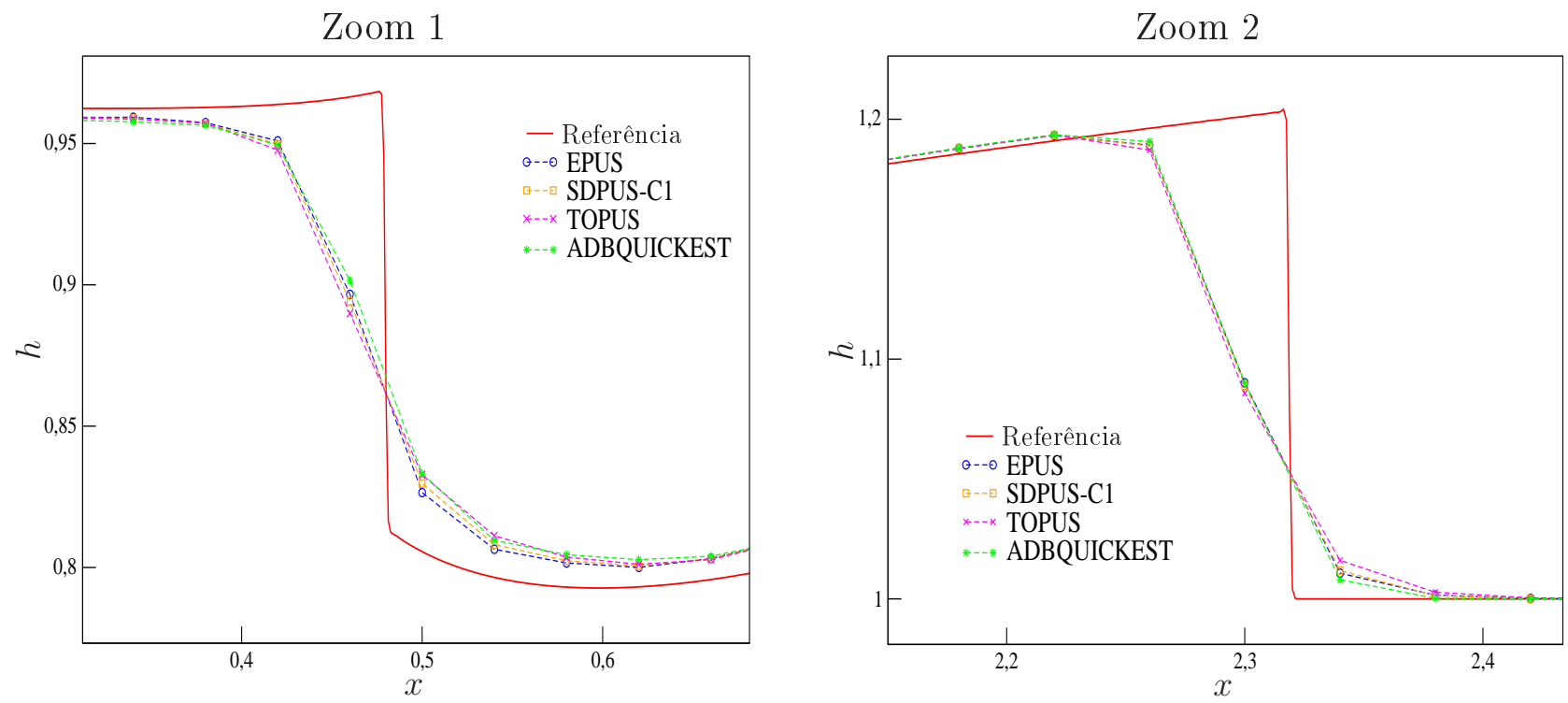

Figura 7.9: regiões de ampliação da Figura 7.8.

\subsection{Escoamentos Incompressíveis Laminares 2D}

Esta seção consiste em verificar o esquema EPUS na resolução de escoamentos incompressíveis laminares 2D com superfícies livres móveis, os quais são modelados pelas equações 2D de Navier-Stokes descritas na Seção 2.3. Para isto, considera-se dois problemas diferentes, a saber, colapso de uma coluna de fluido [12] (conhecido como Broken-dam) e jato livre sobre 
uma superfície rígida impermeável [66]. Estes problemas são descritos e apresentados a seguir. Para a simulação numérica considera-se o esquema EPUS com parâmetro livre $\lambda=95$. O valor para $\lambda=95$ foi considerado aqui em virtude de ter fornecido bons resultados em problemas não lineares complexos envolvendo descontinuidades.

\subsubsection{Colapso de uma Coluna de Fluido}

Este problema é caracterizado por ser um escoamento com superfície livre móvel. Considera-se uma coluna de fluido em equilíbrio hidrostático confinada entre paredes rígidas impermeáveis e sob à ação da gravidade. No tempo $t=0$ o fluido inicia seu movimento como ilustrado na Figura 7.10. Este problema foi estudado originalmente por Martin e Moyce [39], os quais forneceram dados experimentais para o valor máximo do espalhamento horizontal $\left(x_{\max }\right)$. Recentemente, dados numéricos, teóricos e experimentais foram apresentados por Colagrossi e Landrini em [12].

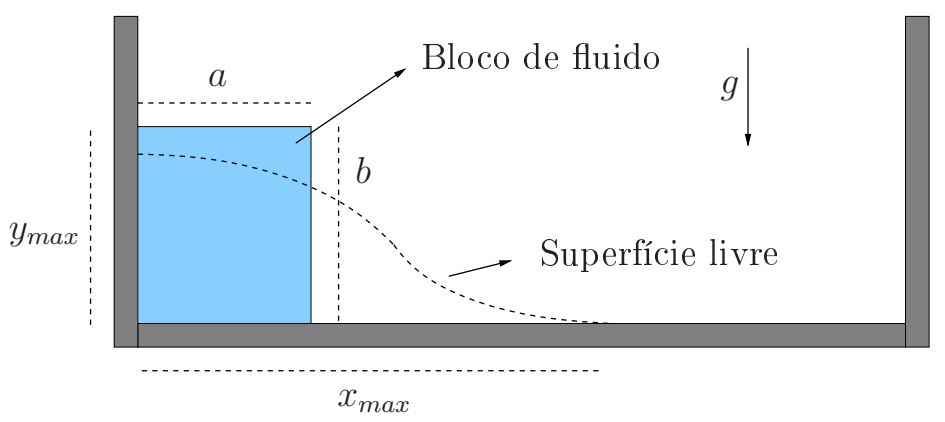

Figura 7.10: ilustração esquemática do problema de colapso de uma coluna de fluido.

Para verificar o desempenho do esquema EPUS neste problema, compara-se o comportamento do espalhamento horizontal $\left(x_{\max }\right)$ calculado com esse esquema com os resultados apresentados por Colagrossi e Landrini [12] e também com o experimento de Martin e Moyce [39]. A condição de contorno aplicada foi a free-slip e os dados utilizados foram:

- Malha: $1000 \times 200$ células computacionais $(\delta x=\delta y=0.0005)$;

- Domínio: $0.5 m \times 0.1 m$;

- Dimensão da coluna de fluido: $a=b=0.057 m$;

- Escala de comprimento: $L_{0}=a=b=0.057 m$;

- Escala de velocidade: $U_{0}=\sqrt{g L_{0}}=0.74778 \mathrm{~m} / \mathrm{s}$;

- Coeficiente de viscosidade cinemática: $\nu=10^{-6} \mathrm{~m}^{2} / \mathrm{s}$;

- Número de Reynolds: $R e=42623.27$.

A Figura 7.11 mostra uma comparação entre os dados experimentais, teóricos e numéricos (extraídos de Colagrossi e Landrini [12]) e os resultados obtidos pelo esquema EPUS para o valor 
$x_{\max }$. A partir desta comparação, pode-se concluir que o novo esquema EPUS fornece resultados em concordância com os dados disponíveis na literatura (numéricos, teóricos e experimentais).

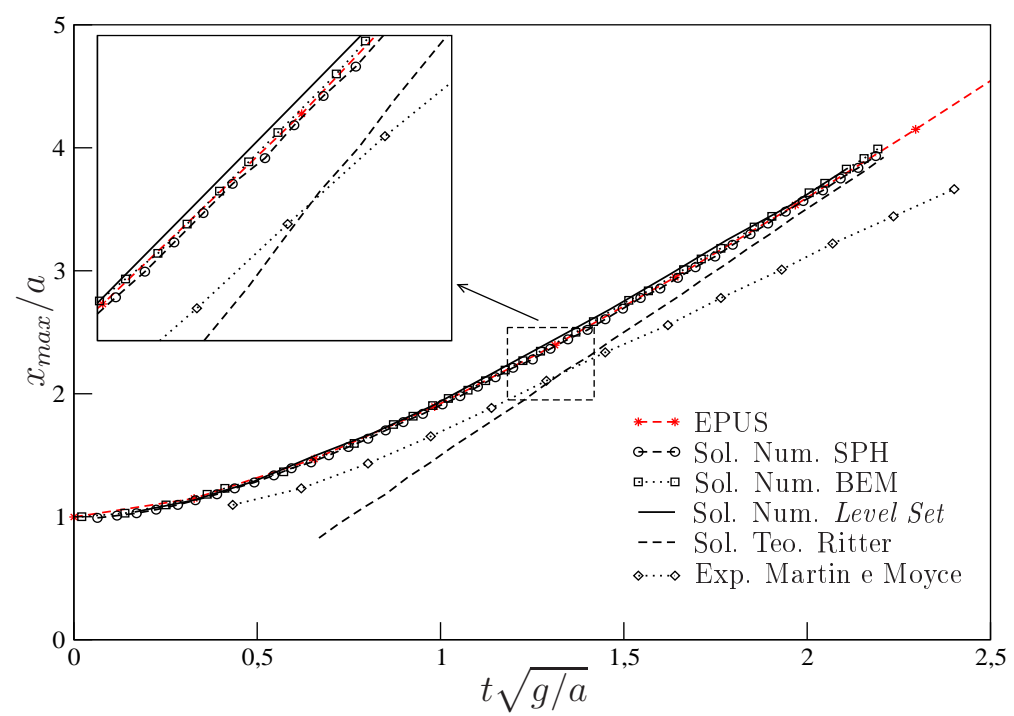

Figura 7.11: comparação das soluções para o problema do colapso de uma coluna de fluido.

Para ilustrar a evolução do escoamento como um todo e da superfície livre, nas Figuras 7.12, 7.13 e 7.14 apresentam-se, respectivamente, os resultados para pressão e velocidades nas direções $x$ e $y$ nos tempos $t=0.05 s, t=0.1 s, t=0.15 \mathrm{~s}$ e $t=0.2 \mathrm{~s}$. Analisando essas figuras, nota-se que de fato o esquema EPUS captura satisfatoriamente o fenômeno do colapso 2D.

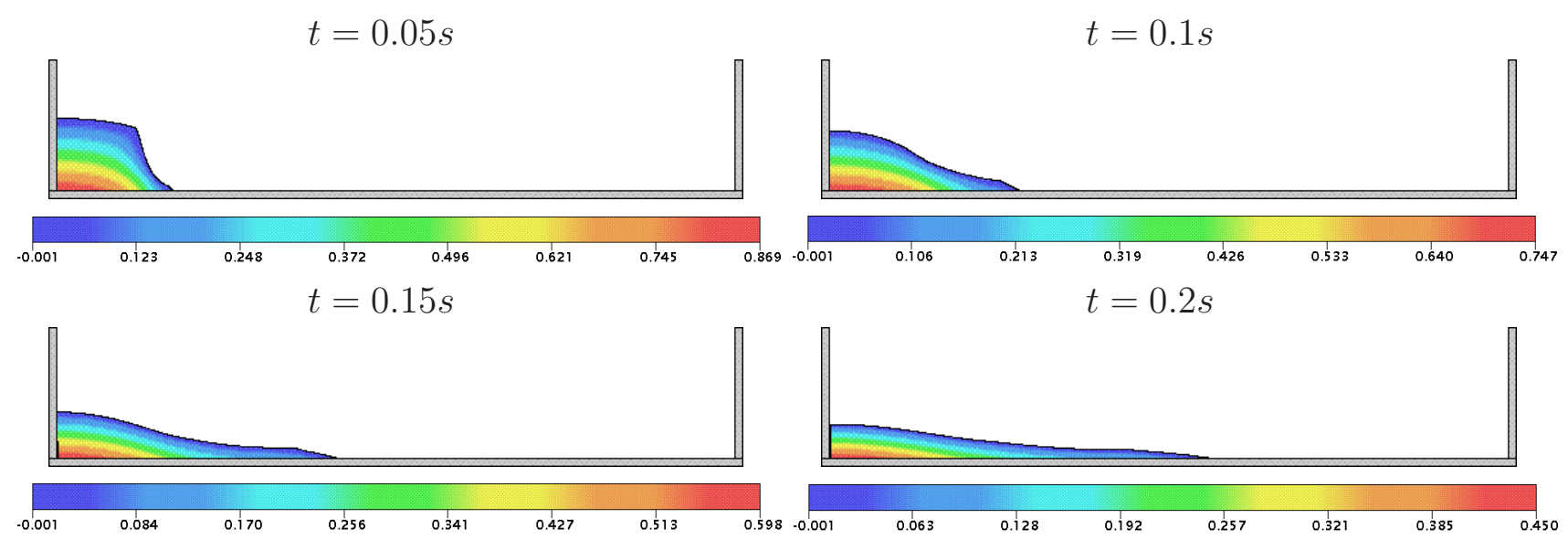

Figura 7.12: contorno da pressão para o problema do colapso de uma coluna de fluido em diferentes tempos. 


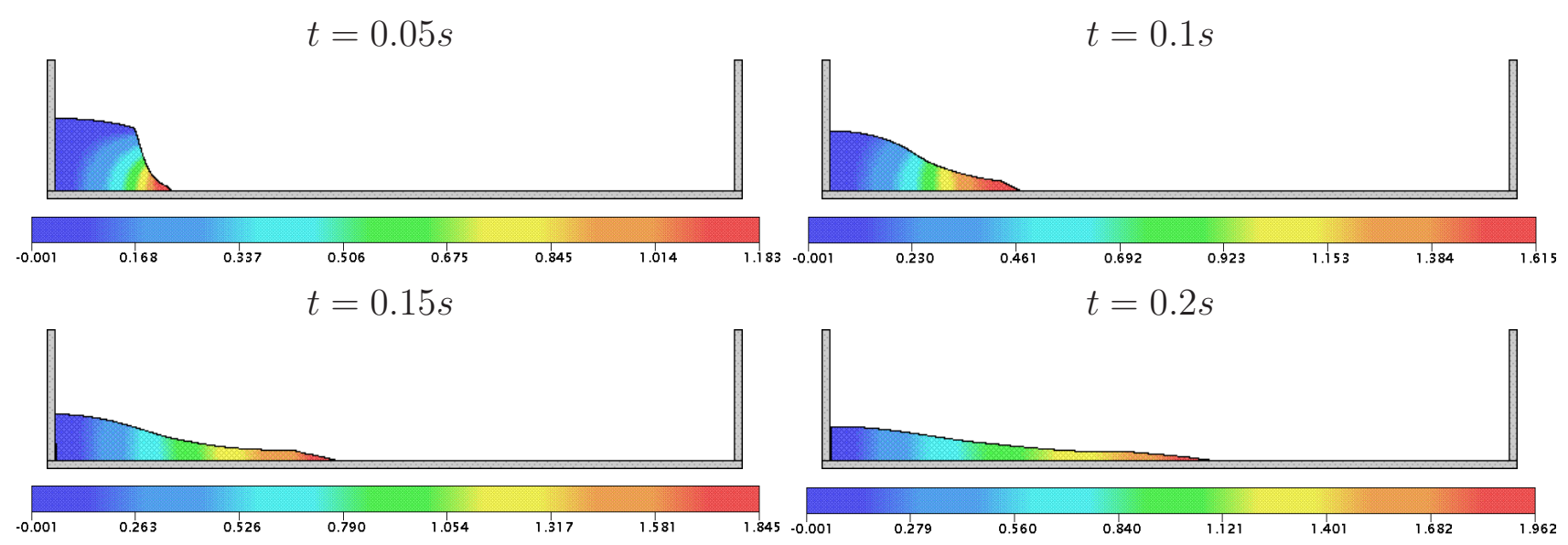

Figura 7.13: contorno da velocidade na direção $x$ para o problema do colapso de uma coluna de fluido em diferentes tempos.

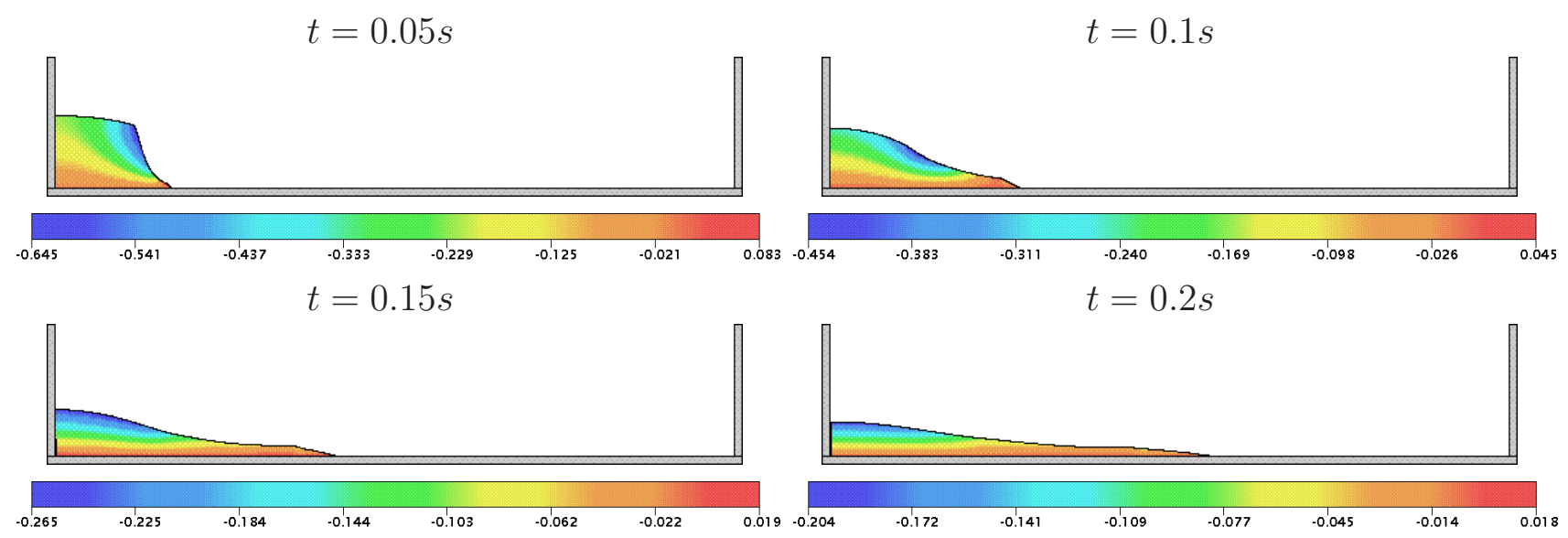

Figura 7.14: contorno da velocidade na direção y para o problema do colapso de uma coluna de fluido em diferentes tempos.

\subsubsection{Jato Livre sobre uma Superfície Rígida Impermeável}

Nesta seção, o esquema EPUS é verificado na resolução numérica do problema do jato livre incidindo perpendicularmente sobre uma superfície rígida impermeável sob a ação da força gravitacional (ver ilustração deste fenômeno na Figura 7.15). Foi proposto por Watson [66] uma solução analítica para a altura $H$ da superfície livre, dada por

$$
H(x)= \begin{cases}\frac{\pi}{\sqrt{3}} \frac{\nu(x+l)}{Q}, & x \geq x_{0}, \\ a+\left(\frac{1-2 \pi}{3 \sqrt{3} c^{2}}\right) \delta(x), & x<x_{0}\end{cases}
$$

em que $Q$ é a vazão, dada por $Q=a U_{0}$, considerando $a=L_{0} / 2$ o raio do injetor e 


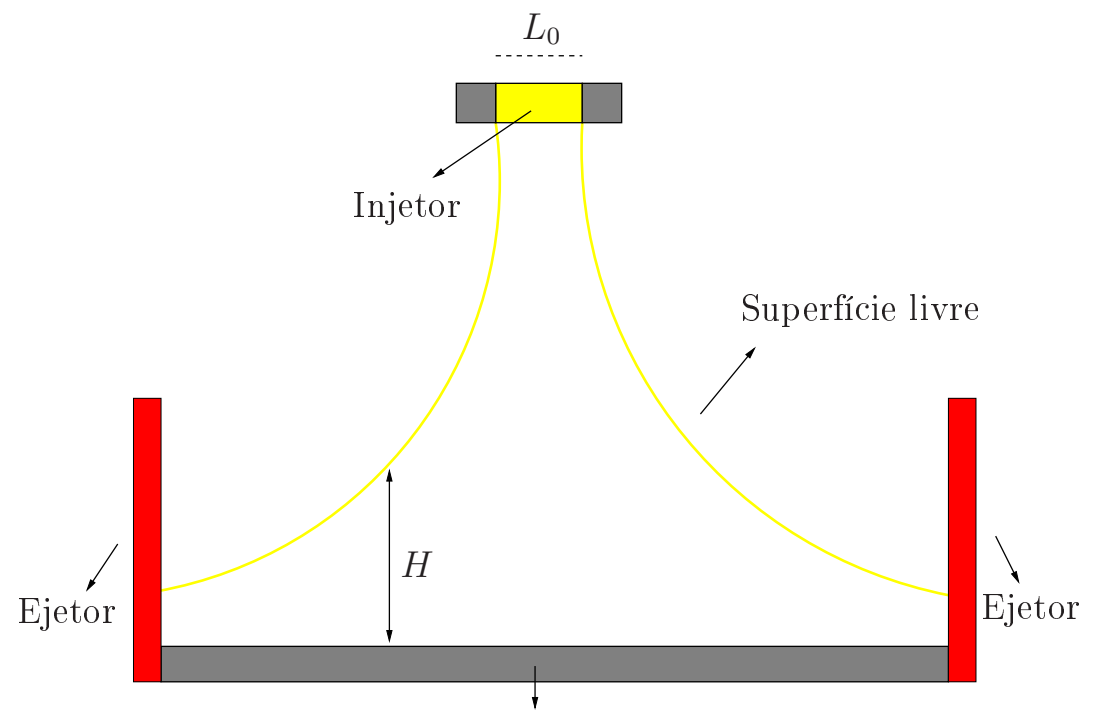

Superfície rígida

Figura 7.15: ilustração esquemática de um jato livre incidindo perpendicularmente sobre uma superfície rígida impermeável.

$$
\begin{gathered}
x_{0}=\left(\frac{3 \sqrt{3} c(\pi-c) \sqrt{3}}{2 \pi^{2}}\right) a R e, \\
l=\left(\frac{3 \sqrt{3} c(2 \sqrt{3} c-\pi)}{2 \pi^{2}}\right) a R e \\
\delta^{2}(x)=\frac{3 \sqrt{3} c^{3}}{2(\pi-c \sqrt{3})} \frac{\nu x}{U_{0}} .
\end{gathered}
$$

Para a simulação deste problema, considera-se condição de contorno no-slip aplicada nas paredes rígidas e os seguintes dados:

- Malha I: $100 \times 25$ células computacionais $(\delta x=\delta y=0.001)$;

- Malha II: $200 \times 50$ células computacionais $(\delta x=\delta y=0.0005)$;

- Malha III: $400 \times 100$ células computacionais $(\delta x=\delta y=0.00025)$;

- Malha IV: $800 \times 200$ células computacionais $(\delta x=\delta y=0.000125)$;

- Raio do injetor: $r_{i}=0.002 m$;

- Domínio: $0.1 \mathrm{~m} \times 0.025 \mathrm{~m}$;

- Escala de comprimento: $L_{0}=2 r_{i}=0.004 m$;

- Escala de velocidade (velocidade de injeção): $U_{0}=1 \mathrm{~m} / \mathrm{s}$;

- Coeficiente de viscosidade cinemática: $\nu=2 \times 10^{-6} \mathrm{~m}^{2} / \mathrm{s}$;

- Número de Reynolds: $R e=2000$. 
A Figura 7.25 apresenta uma comparação entre a solução analítica de Watson e as soluções calculadas pelo esquema EPUS nas quatro malhas I, II, III e IV. Analisando esta figura, pode-se observar que os resultados apresentados pelo novo esquema nas malhas mais finas estão em concordância com a solução analítica de Watson. Ainda, para ilustração deste escoamento com superfície livre, as Figuras 7.17, 7.18 e 7.19 mostram a dinâmica do problema na Malha IV, respectivamente, para os perfis da pressão e das velocidades nas direções $x$ e $y$. Observando estas figuras, pode-se inferir que o esquema EPUS simula satisfatoriamente o problema do jato livre sobre uma superfície rígida impermeável.

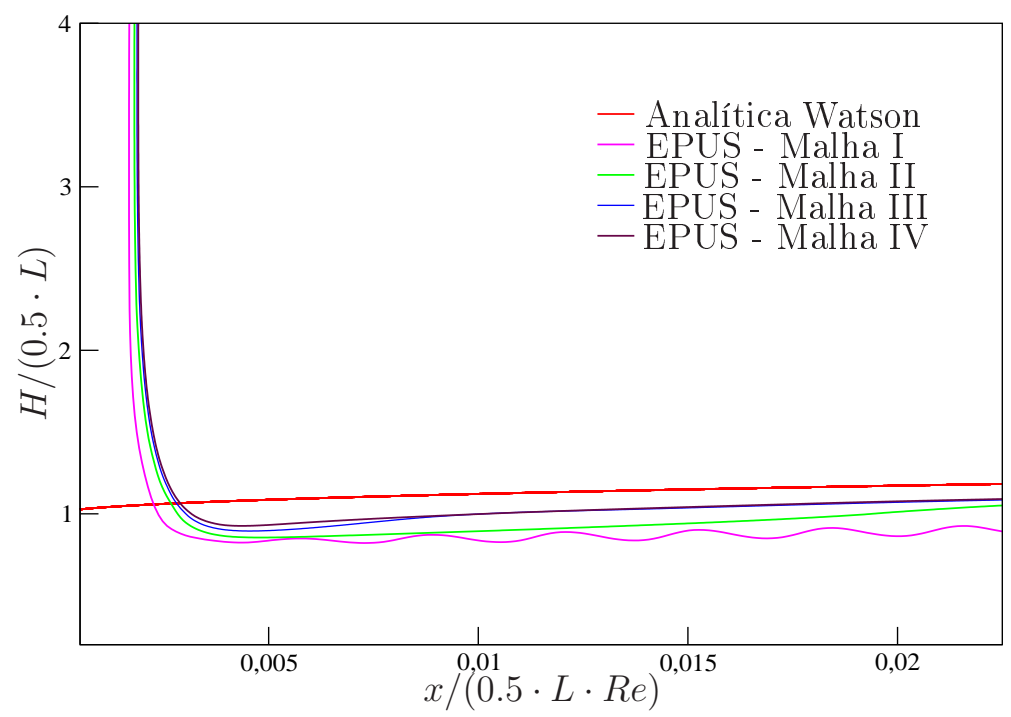

Figura 7.16: comparação das soluções para o problema do jato livre sobre uma superfície rígida impermeável.

\subsection{Escoamentos Incompressíveis Turbulentos 2D}

Esta seção é reservada para verificação do esquema EPUS na resolução de escoamentos incompressíveis 2D em regime turbulento com superfícies livres móveis, os quais são aqui modelados pelas equações médias de Reynolds acopladas ao modelo $\kappa-\varepsilon$ de turbulência (descritas nas Seções 2.4 e 2.5, respectivamente). Para tanto, resolve-se aqui o mesmo problema da Subseção 7.2.2 para o caso do regime turbulento.

Uma solução analítica para este problema foi calculada por Watson [66], que apresenta uma relação para a altura $H$ da superfície livre, dada por

$$
H(x)= \begin{cases}\frac{81(7 A)^{\frac{1}{4}} k}{800}\left(\frac{\nu}{Q}\right)^{\frac{1}{4}}(x+l), & x \geq x_{0}, \\ a+\left(1-\frac{A}{k}\right) \delta(x), & x<x_{0}\end{cases}
$$



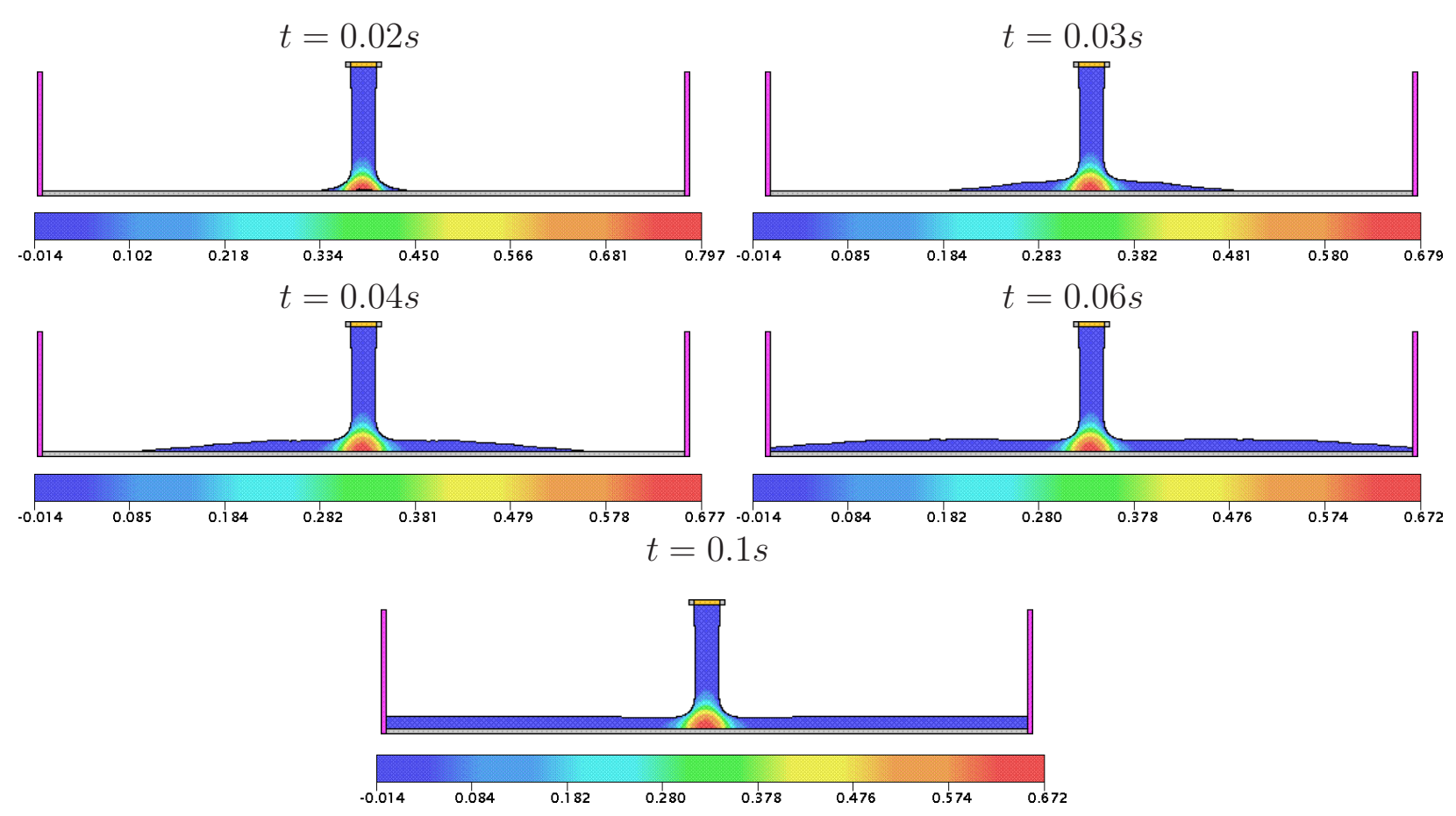

Figura 7.17: contorno da pressão para o problema do jato livre sobre uma superfície rígida impermeável em diferentes tempos.
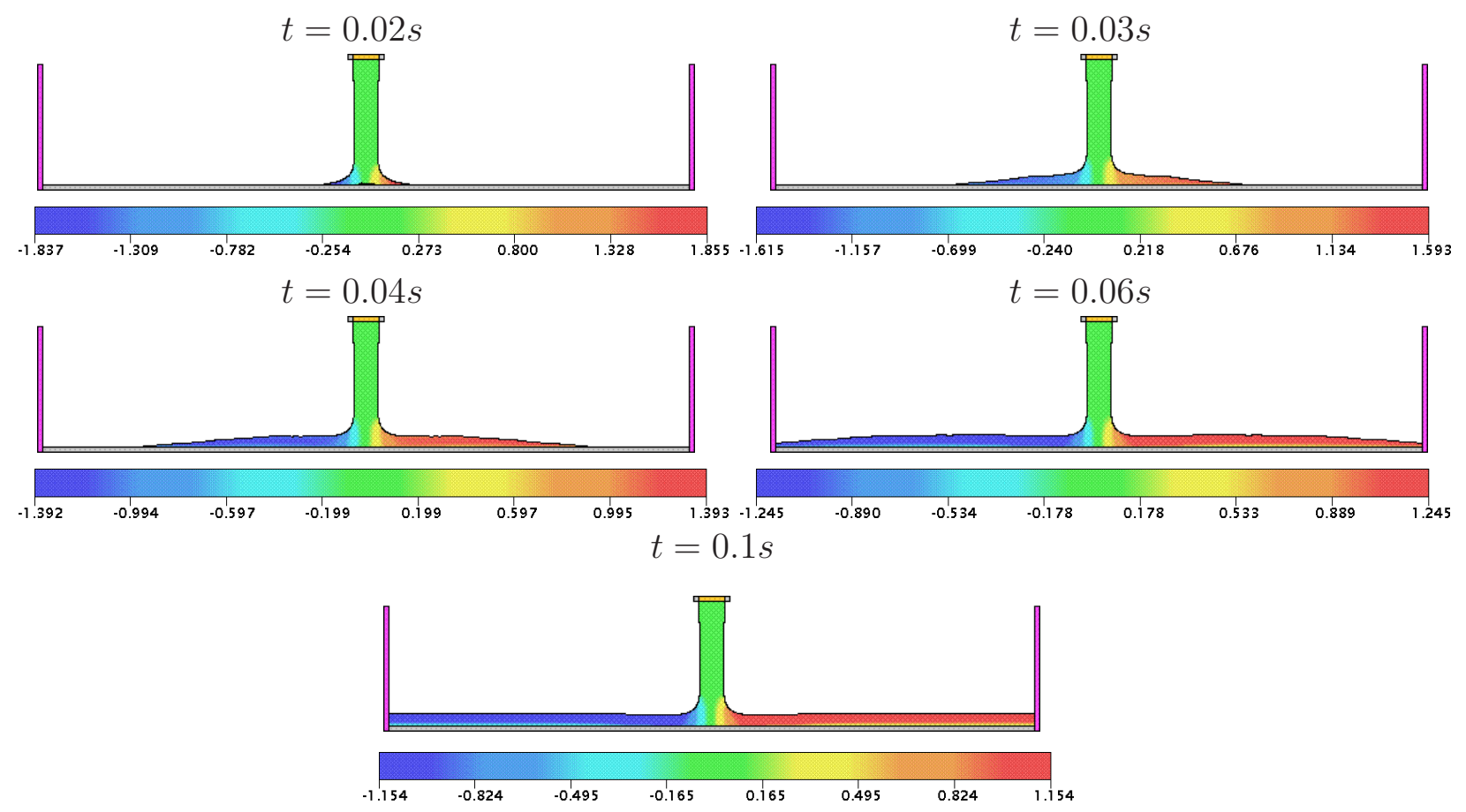

Figura 7.18: contorno da velocidade na direção $x$ para o problema do jato livre sobre uma superfície rígida impermeável em diferentes tempos. 


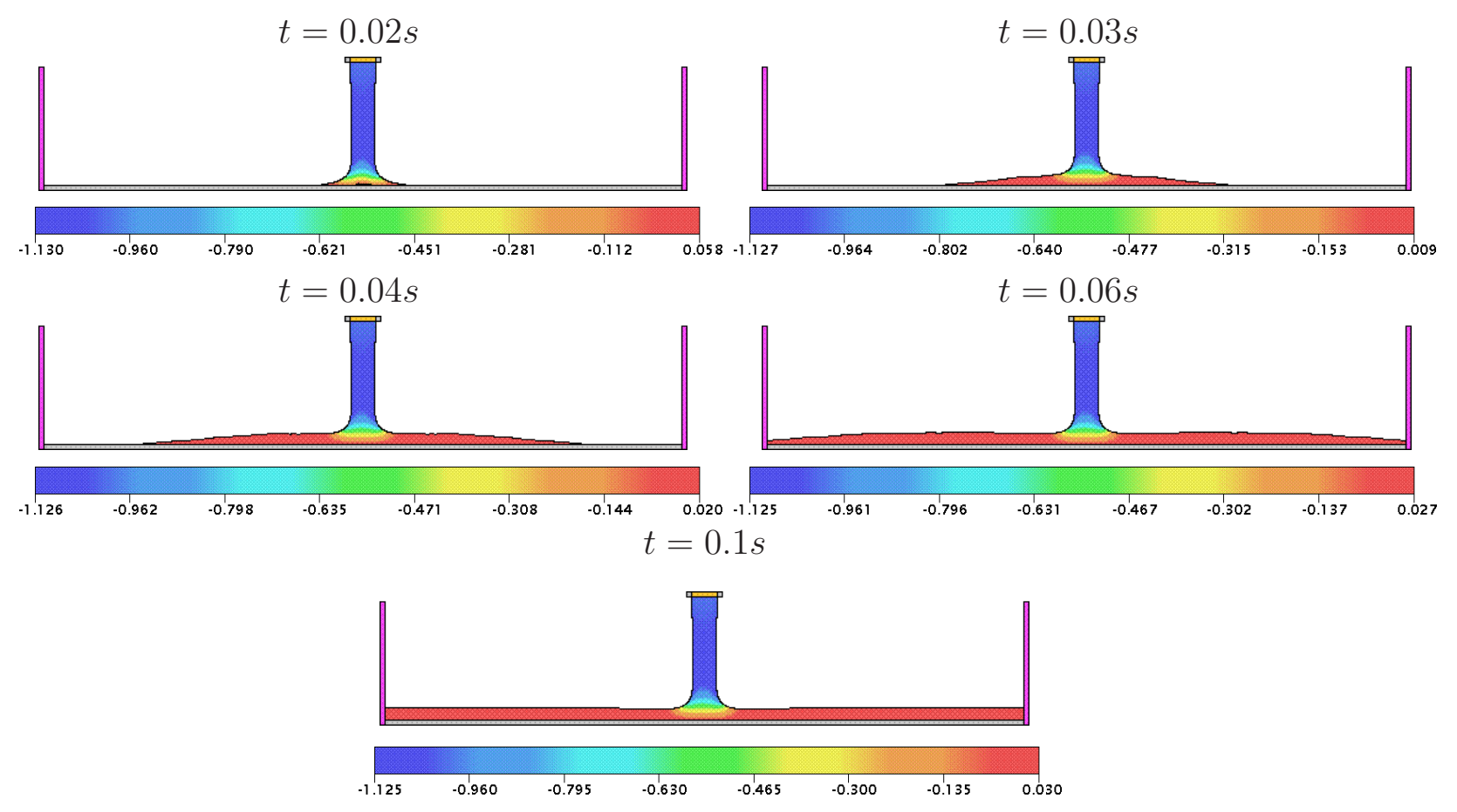

Figura 7.19: contorno da velocidade na direção y para o problema do jato livre sobre uma superfície rígida impermeável em diferentes tempos.

em que $A=0.239$ e $k=0.26$. Ainda, tem-se que

$$
\begin{gathered}
x_{0}=\frac{320(9 A-2)}{81 \times 7^{\frac{1}{4}} A^{\frac{5}{4}}} a R e^{\frac{1}{4}}, \\
l=\frac{160(1-2 A)}{9 \times 7^{\frac{1}{4}} A^{\frac{5}{4}}} a R e^{\frac{1}{4}}, \\
\delta(x)=\left(\frac{81}{320(9 A-2)}\right)^{\frac{4}{5}} 7^{\frac{1}{5}} k\left(\frac{a \nu}{Q}\right)^{\frac{1}{5}} x^{\frac{4}{5}} .
\end{gathered}
$$

Para a simulação deste problema, considera-se condição de contorno no-slip aplicada nas paredes rígidas e os seguintes dados:

- Malha I: $200 \times 50$ células computacionais $(\delta x=\delta y=0.001)$;

- Malha II: $400 \times 100$ células computacionais $(\delta x=\delta y=0.0005)$;

- Malha III: $800 \times 200$ células computacionais $(\delta x=\delta y=0.00025)$;

- Domínio: $0.2 m \times 0.05 m$;

- Raio do injetor: $r_{i}=0.005 m$;

- Escala de comprimento: $L_{0}=2 r_{i}=0.01 m$; 
- Escala de velocidade (velocidade de injeção): $U_{0}=1 \mathrm{~m} / \mathrm{s}$;

- Coeficiente de viscosidade cinemática: $\nu=2 \times 10^{-7} \mathrm{~m}^{2} / \mathrm{s}$;

- Número de Reynolds: $R e=50000$.

A Figura 7.20 mostra os resultados obtidos a partir da simulação feita com o esquema EPUS nas malhas I, II e III, soluções estas que são comparadas com a solução analítica de Watson dada pela Eq. (7.8). O tempo final de simulação considerado é $t=1$ s (tempo em que o escoamento já atingiu o estado estacionário). Analisando esta figura pode-se inferir que o novo esquema apresenta bom desempenho neste teste, mostrando resultados consistentes com a solução analítica de Watson, sendo o melhor deles para a malha mais fina, conforme esperado.
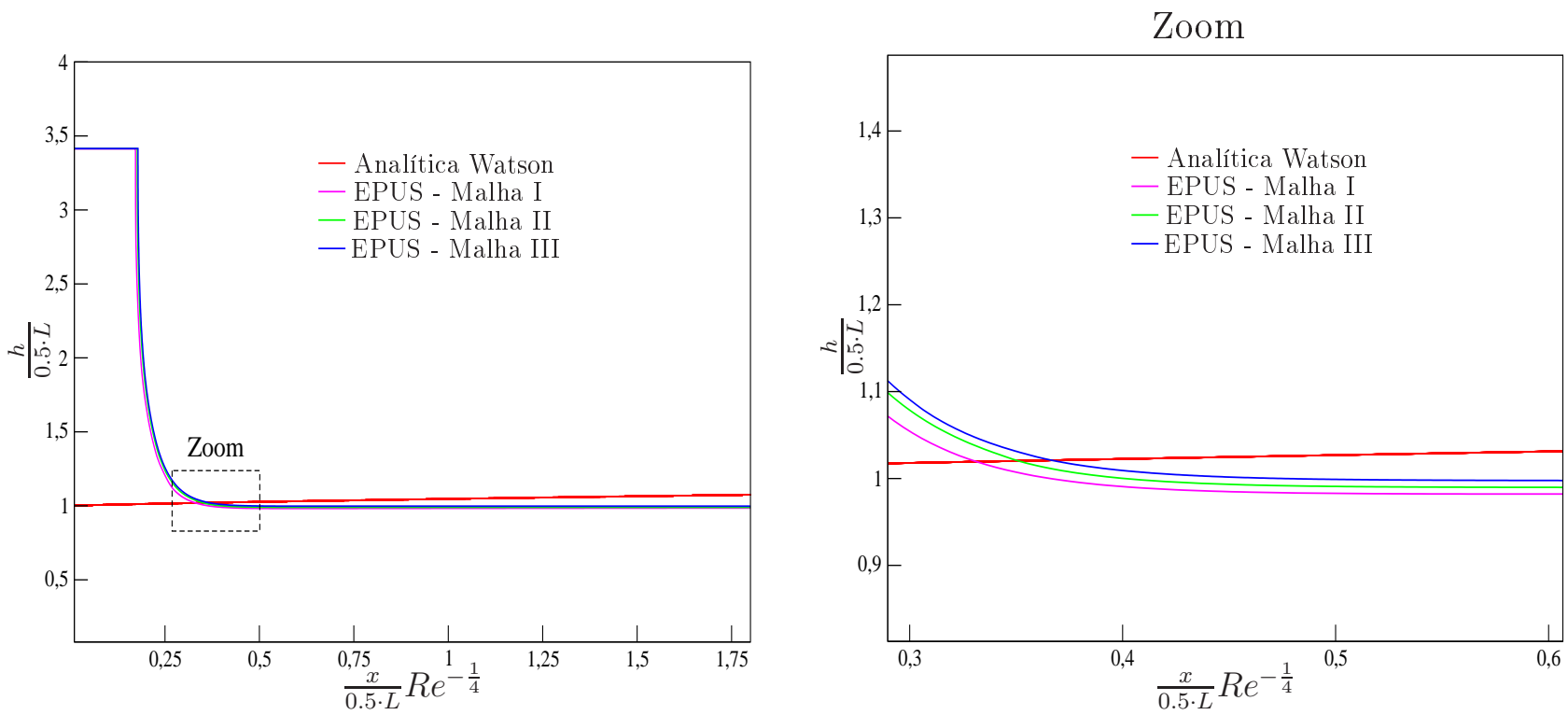

Figura 7.20: comparação das soluções para o problema do jato livre sobre uma superfície rígida impermeável (caso turbulento).

Neste teste também são apresentados os contornos da pressão e das velocidades nas direções $x$ e $y$, os quais são mostrados nas Figuras 7.21, 7.22 e 7.23, respectivamente. A partir destas ilustrações, observa-se claramente que o esquema EPUS captura satisfatoriamente o fenômeno simulado. 


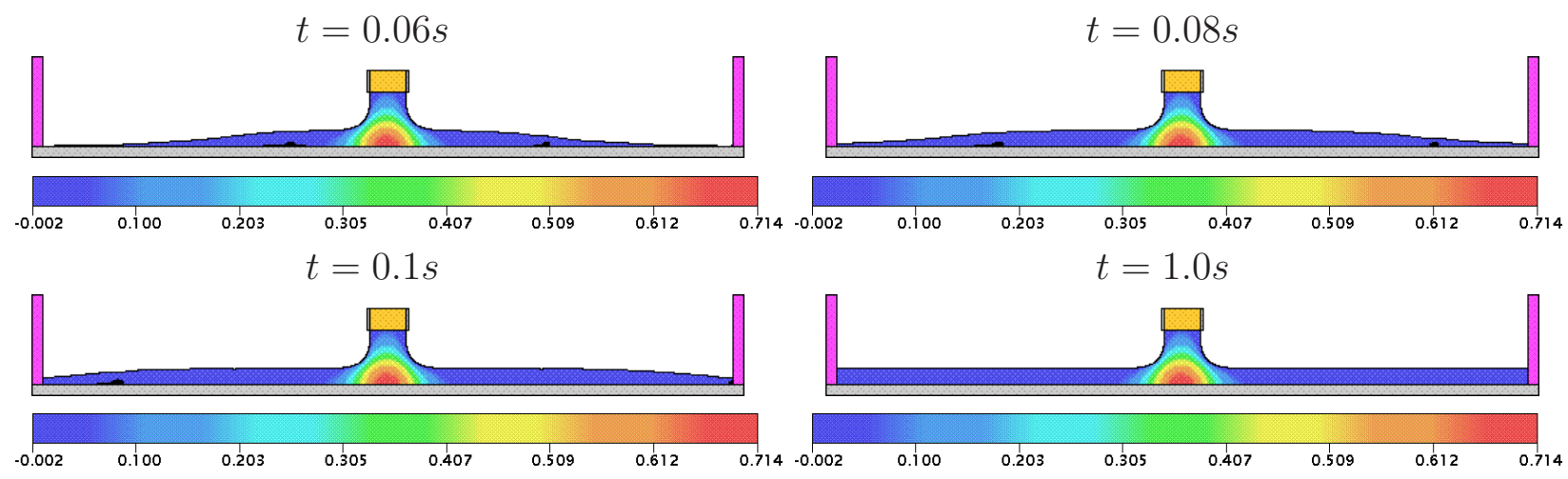

Figura 7.21: contorno da pressão para o problema do jato livre sobre uma superfície rígida impermeável (caso turbulento).

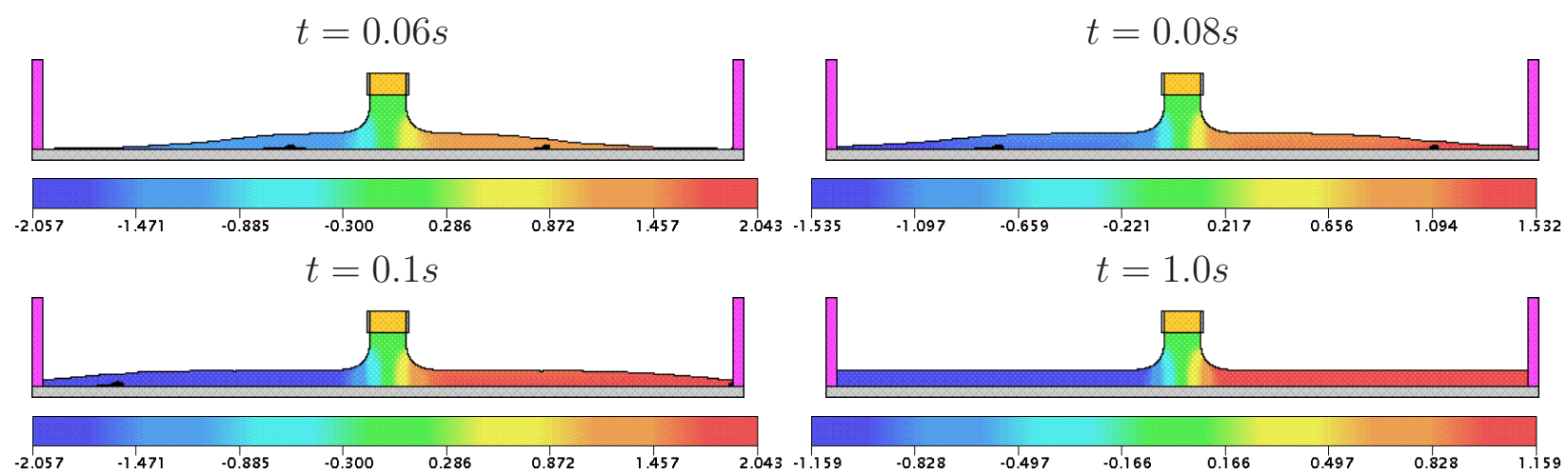

Figura 7.22: contorno da velocidade na direção $x$ para o problema do jato livre sobre uma superfície rígida impermeável (caso turbulento).

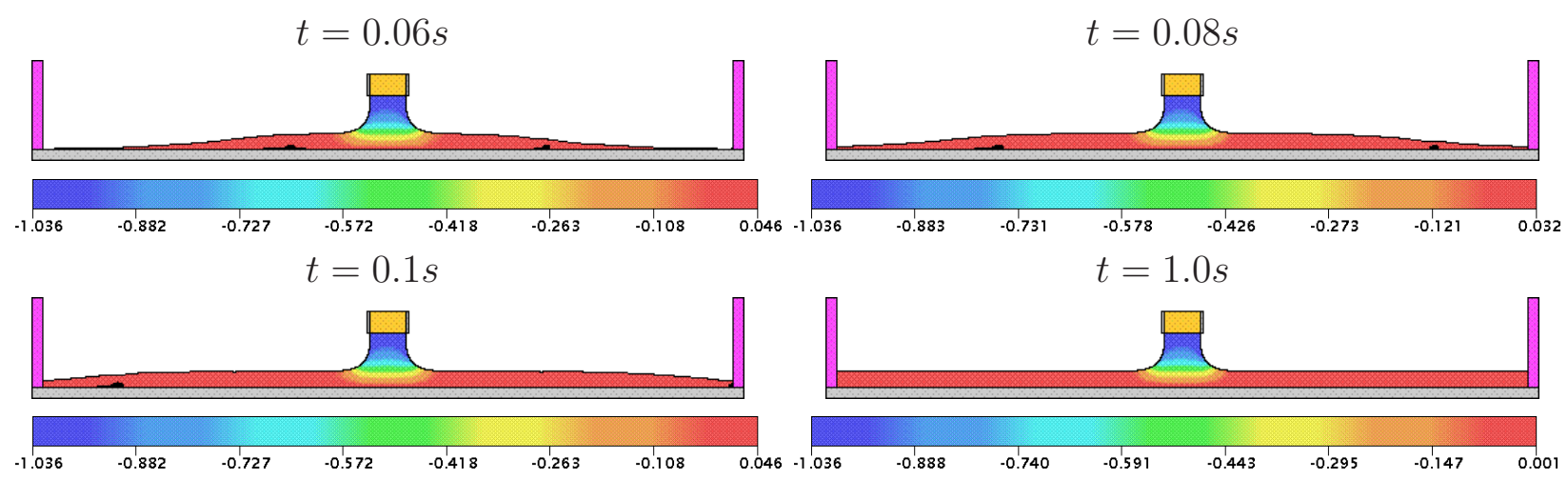

Figura 7.23: contorno da velocidade na direção y para o problema do jato livre sobre uma superfície rígida impermeável (caso turbulento). 


\subsection{Escoamentos Incompressíveis Laminares Axissimétri- $\cos$}

Esta seção consiste em verificar o esquema EPUS na resolução de escoamentos incompressíveis axissimétricos com superfícies livres, os quais são modelados pelas equações axissimétricas de Navier-Stokes, descritas na Seção 2.3. Para isto, considera-se dois problemas diferentes, a saber, ressalto hidráulico circular [66, 6, 8] e experimento de Taylor [57], os quais são descritos e apresentados a seguir, aplicando-se em suas resoluções numéricas o novo esquema EPUS com parâmetro livre $\lambda=95$.

\subsubsection{Ressalto Hidráulico Circular}

Este problema consiste em um jato vertical livre incidindo perpendicularmente sobre uma superfície rígida impermeável (sob à ação do campo gravitacional), levando à formação do curioso fenômeno observável na vida cotidiana, o qual é conhecido como ressalto hidráulico circular (ver ilustração deste fenômeno na Figura 7.24).

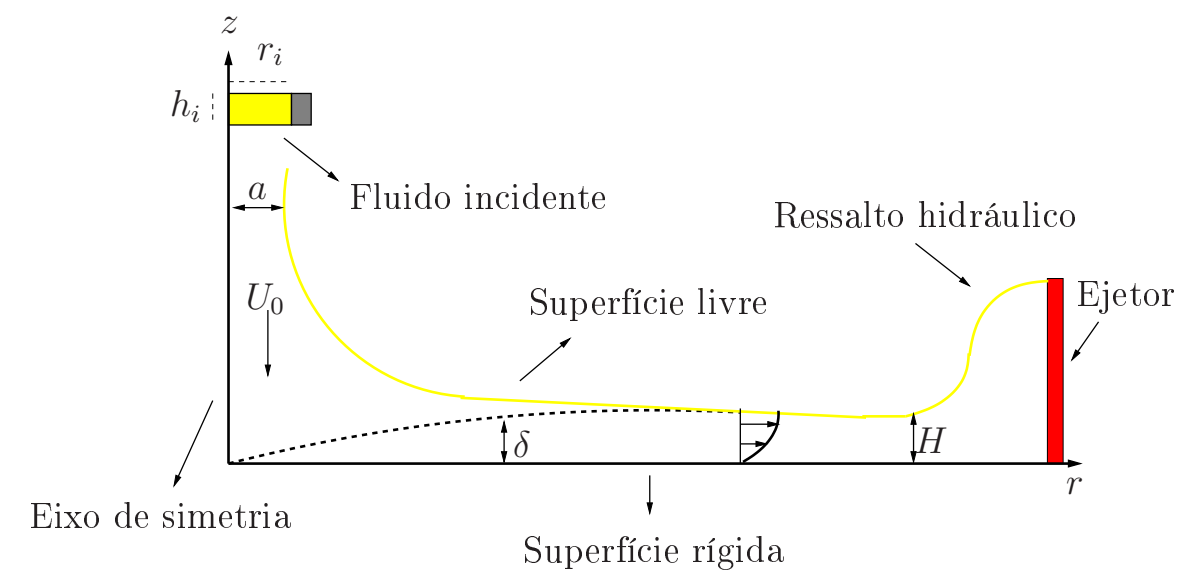

Figura 7.24: ilustração esquemática do ressalto hidráulico circular.

Uma solução analítica viscosa para este problema foi calculada por Watson [66], e é dada por

$$
H(r)= \begin{cases}\frac{a^{2}}{2 r}+\left(1-\frac{2 \pi}{3 \sqrt{3} c^{2}}\right) \delta, & r<r_{0} \\ \frac{2 \pi^{2}}{3 \sqrt{3}} \frac{\nu\left(r^{3}+l^{2}\right)}{Q r}, & r \geq r_{0}\end{cases}
$$

em que

$$
\delta^{2}=\frac{\pi \sqrt{3} c^{3}}{\pi-c \sqrt{3}} \frac{\nu r a^{2}}{Q}
$$

onde $c=1.402, r_{0}=0.3155 a R e^{\frac{1}{3}}$ e $l=0.567 a R e^{\frac{1}{3}}$ é uma constante arbitrária, a qual é 
calculada considerando o desenvolvimento inicial da camada limite. Vale ressaltar que esta solução analítica é válida somente na região do domínio depois que a superfície livre encontrou a camada limite e antes da formação do ressalto hidráulico (mais detalhes sobre esta solução ver Watson [66]).

Para a simulação computacional deste problema, utiliza-se condição de contorno no-slip e os seguintes dados:

- Malha I: $800 \times 504$ células computacionais $(\delta x=\delta y=0.0000625)$;

- Malha II: $400 \times 252$ células computacionais $(\delta x=\delta y=0.000125)$;

- Malha III: $200 \times 126$ células computacionais $(\delta x=\delta y=0.00025)$;

- Domínio: $0.050 m \times 0.0315 m$;

- Raio do injetor: $r_{i}=0.004 m$;

- Altura do injetor: $h_{i}=0.00075 m$;

- Escala de comprimento: $L_{0}=2 r_{i}=0.008 m$;

- Escala de velocidade (velocidade de injeção): $U_{0}=0.375 \mathrm{~m} / \mathrm{s}$;

- Coeficiente de viscosidade cinemática: $\nu=1.2 \cdot 10^{-5} \mathrm{~m}^{2} / \mathrm{s}$;

- Número de Reynolds: $R e=250$.

A Figura 7.25 mostra os resultados para a altura $H$ da superfície livre obtidos com o EPUS nas três malhas I, II e III e a solução analítica de Watson no tempo $t=2.5 \mathrm{~s}$. Esta figura também apresenta, para ilustração, a espessura da camada limite $\delta$ calculada por Watson. A partir desta figura, pode-se inferir que as soluções obtidas pelo esquema EPUS estão em boa concordância com a solução analítica de Watson (na região onde esta é válida).

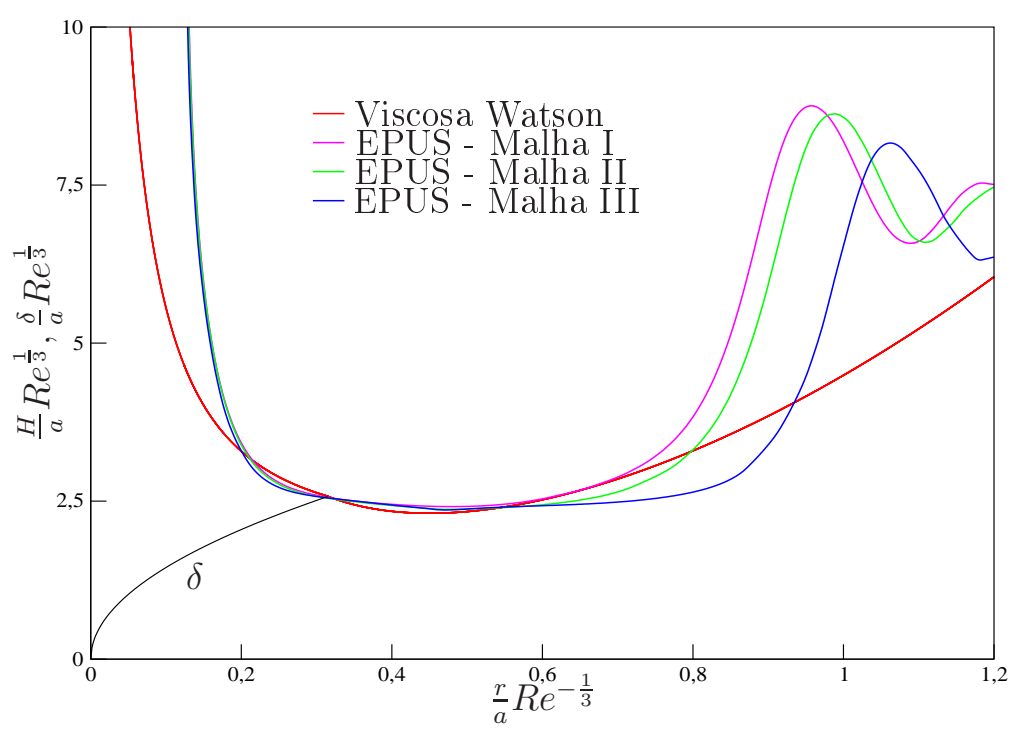

Figura 7.25: comparação das soluções para o problema do ressalto hidráulico circular.

Neste teste compara-se também a altura $H$ obtida com o esquema EPUS na Malha II 
para valores diferentes do espaçamento temporal $\delta t$. Objetiva-se observar a independência dos resultados com a mudança do passo temporal. As soluções obtidas para esta comparação são apresentadas na Figura 7.26, a partir da qual é possível observar que os resultados obtidos com o esquema EPUS mantêm praticamente o mesmo comportamento para diferentes valores de $\delta t$.

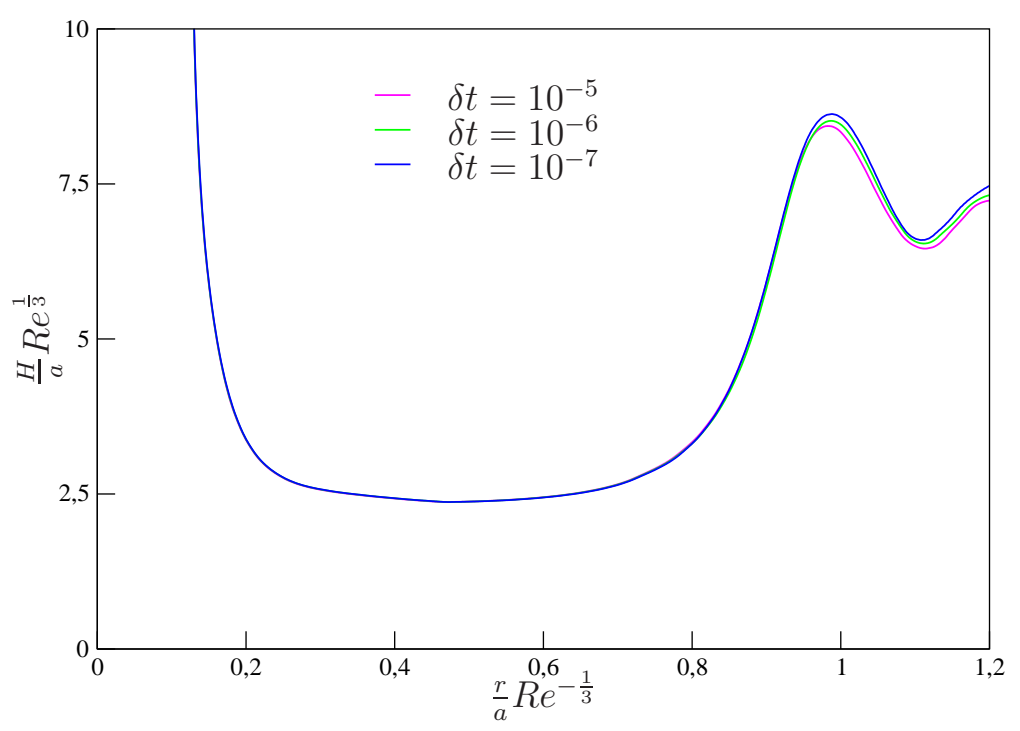

Figura 7.26: comparação das soluções para o problema do ressalto hidráulico circular utilizando diferentes valores para $\delta t$.

Como comparação qualitativa, a Figura 7.27 mostra as visualizações do experimento apresentado em Rai [46] (Figura 7.27 (a)) e da simulação com o esquema EPUS (Figura 7.27 (b)). Vê-se claramente por essa figura que o fenômeno do salto hidráulico circular foi simulado com sucesso pelo esquema EPUS.

(a)

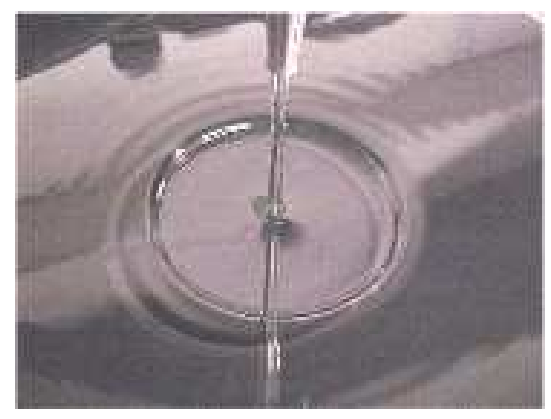

(b)

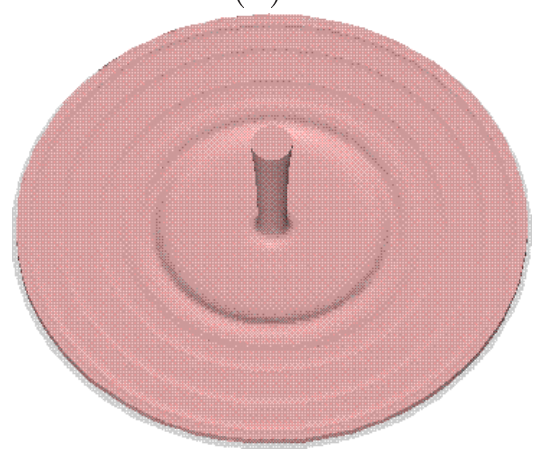

Figura 7.27: ilustração do ressalto hidráulico circular: resultados (a) experimental e (b) simulado pelo esquema EPUS.

Uma análise quantitativa é feita também neste teste. Para tanto, comparam-se os valores do raio do ressalto hidráulico circular calculados com o esquema EPUS e a aproximação teórica 
de Brechet e Néda [8], dada por

$$
R=\left(\frac{27 g^{-1 / 4}}{2^{1 / 4} 35 \pi}\right)^{\frac{2}{3}} Q^{2 / 3} d^{-1 / 6} \nu^{-1 / 3},
$$

em que $d$ é a altura do domínio entre a superfície rígida e o injetor. Os valores obtidos nesta análise seguem apresentados na Tabela 7.2, a partir da qual é possível concluir que conforme refina-se a malha, o raio do ressalto calculado pelo esquema EPUS vai convergindo para a aproximação teórica.

\begin{tabular}{ccc}
\hline \hline \multicolumn{3}{c}{ Raio do Ressalto $(R)$} \\
\hline Malhas & Aproximação Teórica & EPUS \\
\hline Malha-I & $1.325158 \mathrm{e}-2$ & $1.783112 \mathrm{e}-2$ \\
Malha-II & $1.325158 \mathrm{e}-2$ & $1.638573 \mathrm{e}-2$ \\
Malha-III & $1.325158 \mathrm{e}-2$ & $1.414912 \mathrm{e}-2$ \\
\hline \hline
\end{tabular}

Tabela 7.2: comparação dos valores obtidos para o raio do ressalto hidráulico circular.

Finaliza-se este teste mostrando a ordem de convergência observada com o EPUS. Para isso utiliza-se as malhas I, II e III e a relação matemática de Jameson e Martinelli [25]. O valor calculado é

$$
\log _{2}\left|\frac{R_{\text {MalhaI }}-R_{\text {MalhaII }}}{R_{\text {MalhaII }}-R_{\text {MalhaIII }}}\right|=1.90 .
$$

Nota-se que este valor da ordem observada neste problema complexo está consistente com a ordem de precisão formal do esquema EPUS.

\subsubsection{Experimento de Taylor}

Esse experimento consiste num jato vertical incidindo perpendicularmente sobre um recipiente contendo o mesmo fluido em repouso (ver esquema representativo deste problema na Figura 7.28). Este fenômeno, realizado experimentalmente por Taylor [57], também é modelado pelas equações axissimétricas de Navier-Stokes. Na sequência apresenta-se uma comparação qualitativa da simulação com o esquema EPUS e o experimento de Taylor.

Nesta simulação considera-se que o injetor é posicionado a $0.1 \mathrm{~m}$ do fluido em repouso. Além disso, utiliza-se condição de contorno no-slip e os seguintes dados:

- Malha: $123 \times 403$ células computacionais $(\delta x=\delta y=0.0005)$;

- Domínio: $0.0615 m \times 0.2015 m$;

- Raio do injetor: $r_{i}=0.002 m$;

- Altura do injetor: $h_{i}=0.03 m$; 


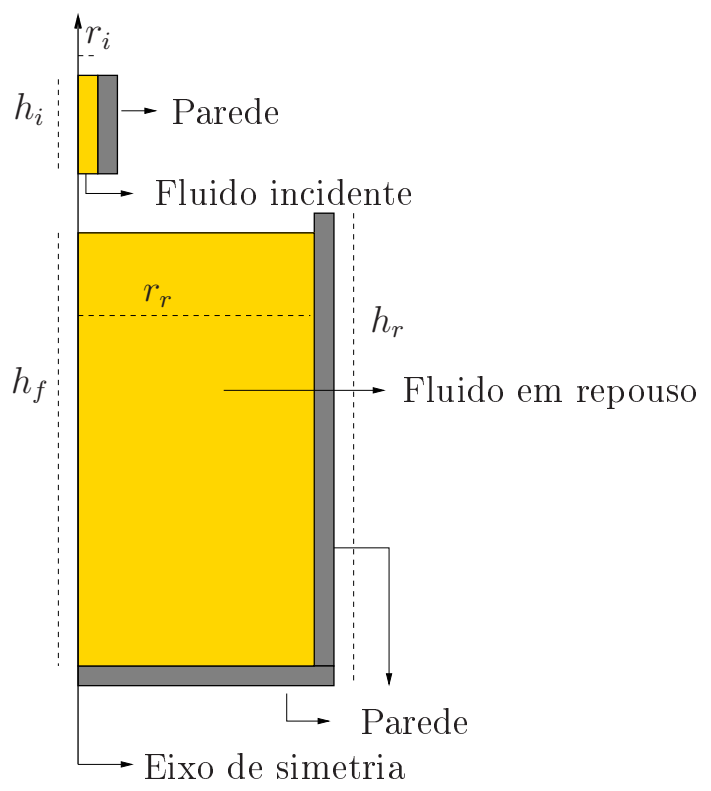

Figura 7.28: ilustração esquemática do experimento de Taylor.

- Raio do recipiente cilíndrico: $r_{r}=0.06 m$;

- Altura do recipiente cilíndrico: $h_{r}=0.17 m$;

- Altura do fluido contido no recipiente: $h_{f}=0.16 m$;

- Escala de comprimento: $L_{0}=2 r_{i}=0.004 m$;

- Escala de velocidade (velocidade de injeção): $U_{0}=0.5 \mathrm{~m} / \mathrm{s}$;

- Coeficiente de viscosidade cinemática: $\nu=10^{-5} \mathrm{~m}^{2} / \mathrm{s}$;

- Número de Reynolds: $R e=200$.

A Figura 7.29 apresenta os resultados obtidos por Taylor em seu experimento e os resultados obtidos pelo esquema EPUS nos tempos $t=0.75 \mathrm{~s}$ e $t=2.5 \mathrm{~s}$. A partir desta figura observa-se que o novo esquema captura satisfatoriamente o fenômeno (estrutura toroidal), mostrando concordância bastante razoável com o experimento de Taylor. Para simples ilustração, na Figura 7.30 apresentam-se a solução obtida pelo EPUS no tempo $t=10 \mathrm{~s}$ e um corte transversal da mesma para visualização das estruturas vorticais. Na Figura 7.31 apresentam-se os resultados para os campos de pressão e velocidades nas direções $r$ e $z$ no tempo $t=10 \mathrm{~s}$. 


$$
t=0.75 s
$$

(a)

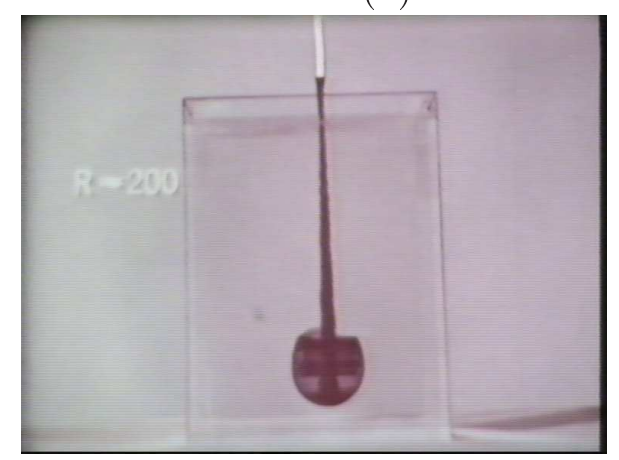

$t=2.5 \mathrm{~s}$

(a)

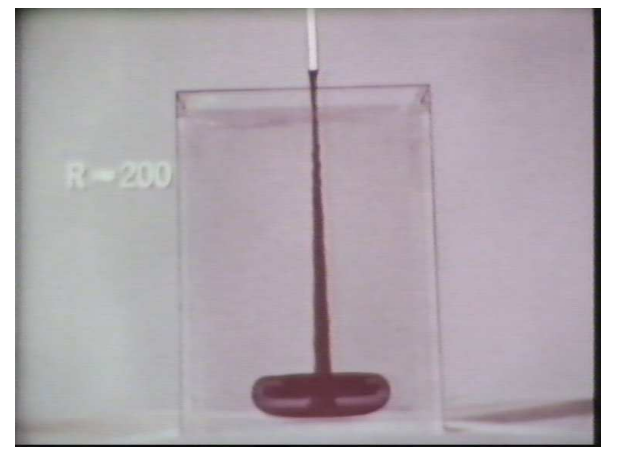

(b)

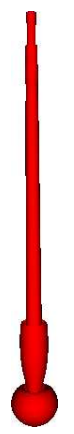

(b)

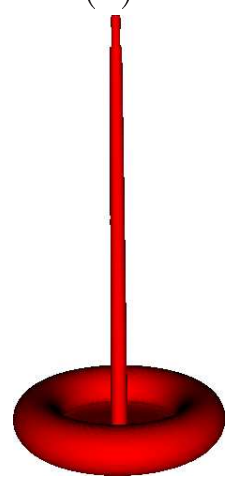

Figura 7.29: ilustração do experimento de Taylor: resultados (a) experimental e (b) simulado pelo esquema EPUS em diferentes tempos.

$$
t=10 \mathrm{~s}
$$

(a)

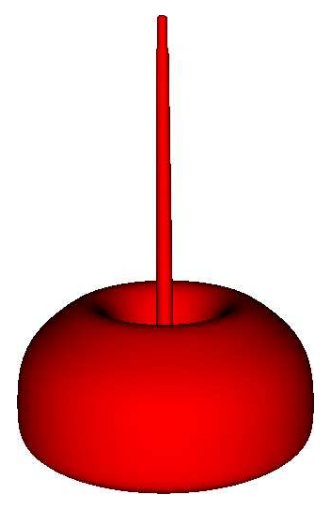

(b)

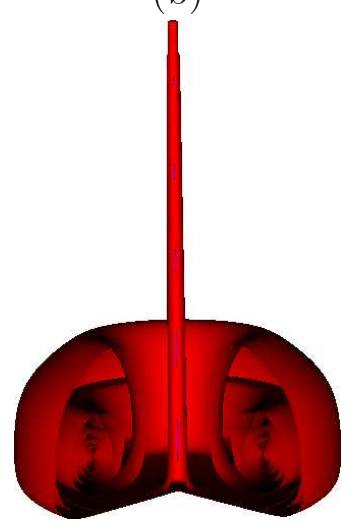

Figura 7.30: ilustração dos resultados obtidos pelo esquema EPUS (corte transversal em (b) para mostrar a estrutura vortical). 
(a)

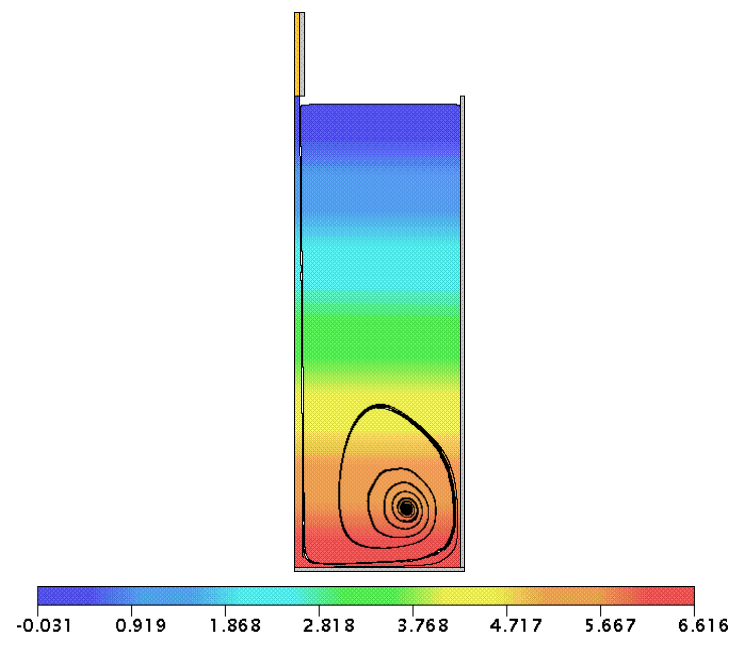

(b)

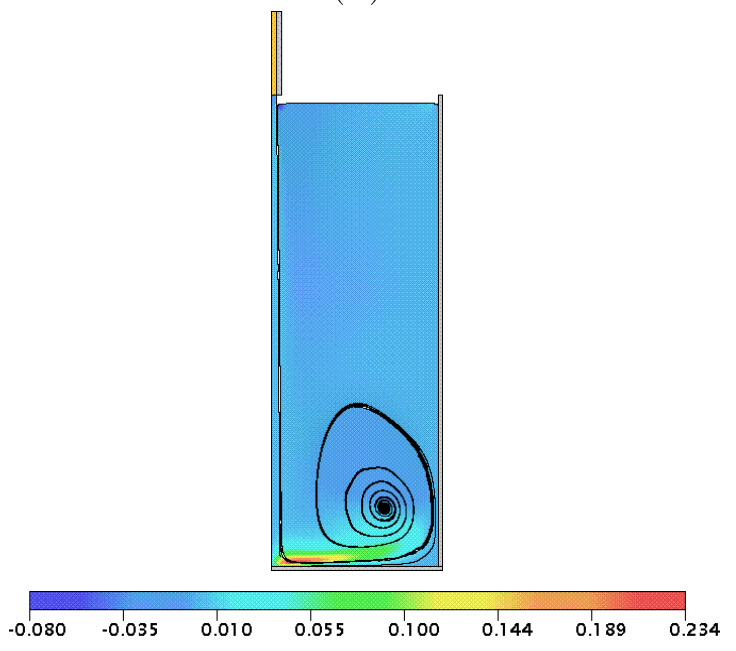

(c)

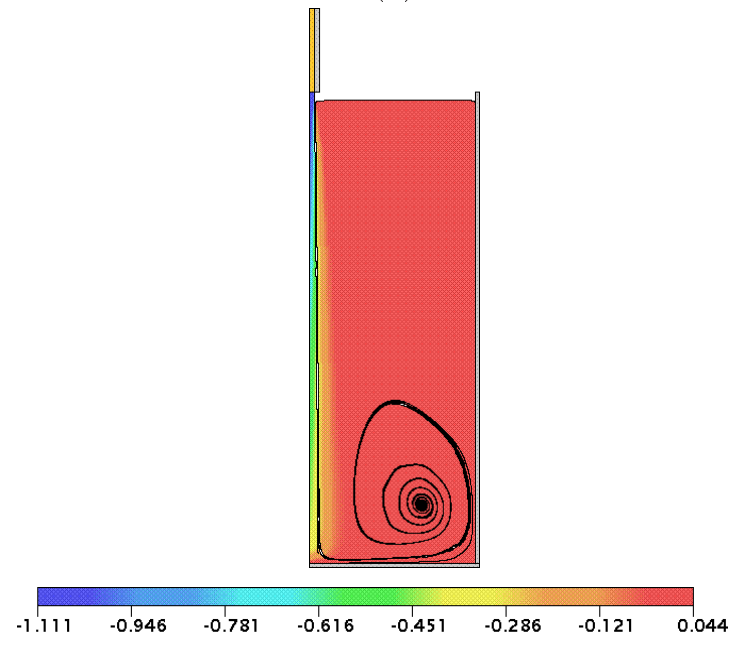

Figura 7.31: experimento de Taylor simulado pelo esquema EPUS: perfis de (a) pressão, (b) velocidade na direção $x$ e (c) velocidade na direção $y$ no tempo $t=10 s$. 


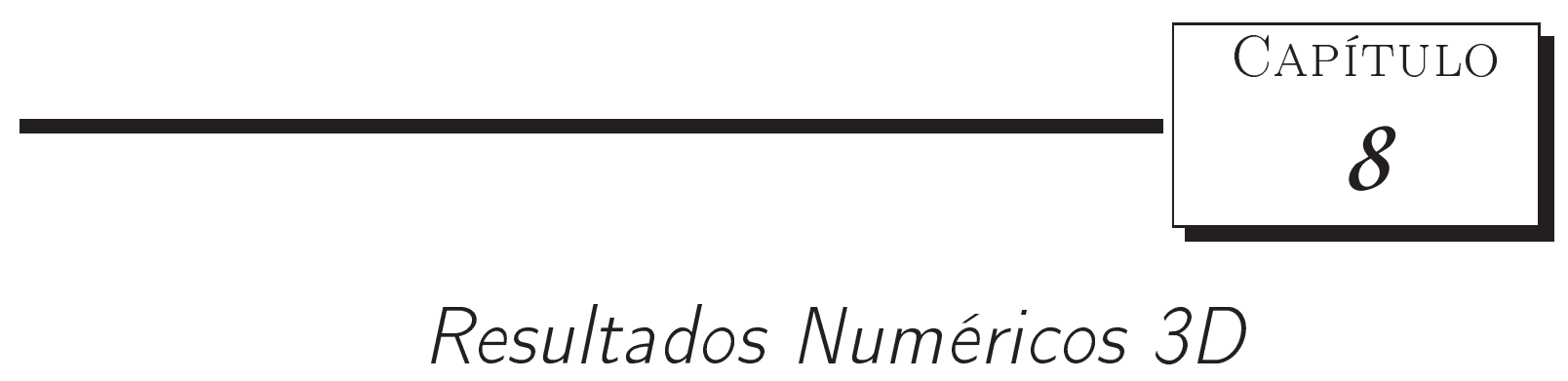

Neste capítulo, o esquema EPUS é investigado na solução das equações de Navier-Stokes para a simulação direta de escoamentos incompressíveis 3D com superfícies livres móveis. Para avaliar os resultados obtidos com o esquema, fazem-se comparações quantitativas e qualitativas contra resultados teóricos, numéricos e experimentais consagrados na literatura. Para isto, consideram-se os seguintes problemas: o do colapso de um bloco de fluido (Broken-dam), o do ressalto hidráulico circular e dos jatos oscilantes (Jet Buckling) nas configurações circular e planar. Na simulação destes problemas tridimensionais não estacionários e com superfície livre utiliza-se o esquema EPUS com $\lambda=95$.

\subsection{Colapso de um bloco de fluido}

Este problema, já apresentado na Subseção 7.2.1 para o caso 2D, é resolvido aqui para o caso 3D. As soluções numéricas obtidas com o esquema EPUS são comparadas com as soluções numéricas, teóricas e experimentais apresentadas por Colagrossi e Landrini [12].

Teste-1: neste teste, simula-se o problema do colapso de um bloco de fluido apresentado na Figura 8.1. Este problema é muito útil em engenharia para o entendimento do escoamento de água em barragens (ver, por exemplo, [3]).

Para esta simulação consideram-se condição de contorno free-slip aplicada nas paredes rígidas e os seguintes dados:

- Malha: $150 \times 50 \times 80$ células computacionais $(\delta x=\delta y=\delta z=0.002)$;

- Domínio: $0.3 m \times 0.1 m \times 0.16 m$;

- Escala de comprimento: $L_{0}=0.1 \mathrm{~m}$; 


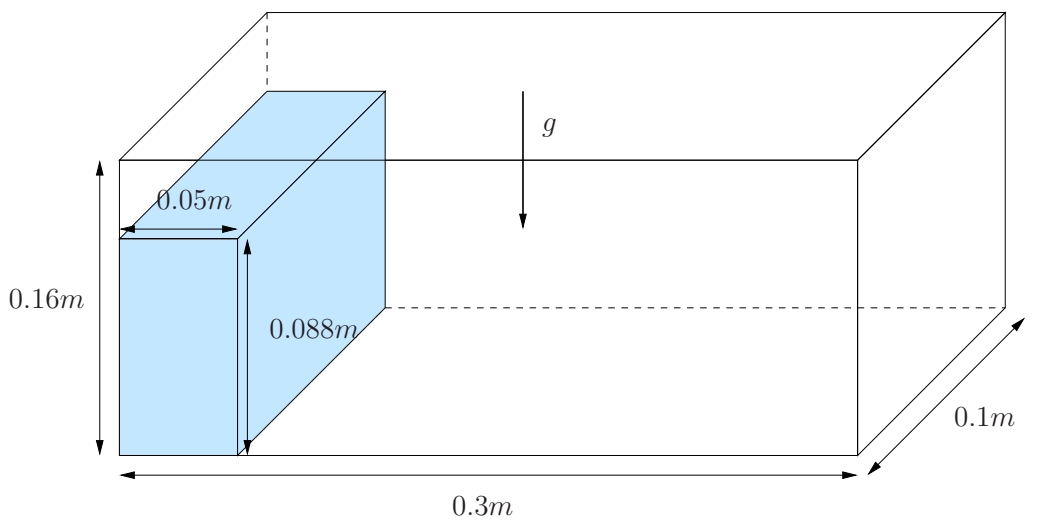

Figura 8.1: ilustração esquemática para o problema do colapso de um bloco de fluido.

- Escala de velocidade: $U_{0}=\sqrt{g L_{0}}=0.99045444 \mathrm{~m} / \mathrm{s}$;

- Coeficiente de viscosidade cinemática: $\nu=10^{-6} \mathrm{~m}^{2} / \mathrm{s}$;

- Número de Reynolds: $R e=99045.444$.

Na Figura 8.2, a solução numérica obtida com o esquema EPUS para o espalhamento horizontal $\left(x_{\max }\right)$ é comparada com os dados apresentados por Colagrossi e Landrini [12]. A partir desta figura, pode-se observar que o novo esquema apresenta resultados satisfatórios, mostrando concordância com os dados da literatura. Em particular, os dados numéricos com o EPUS concordam muito bem com dados numéricos de BEM e Level Set.

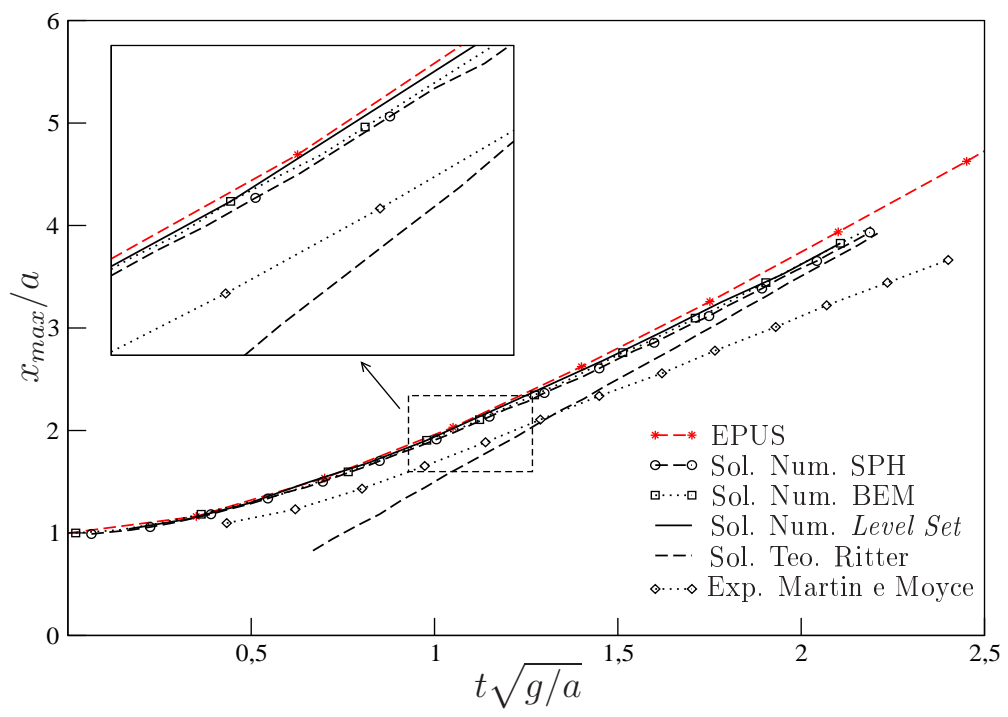

Figura 8.2: comparação das soluções para o problema do colapso de um bloco de fluido.

Para ilustração, nas Figuras 8.3, 8.4 e 8.5 são apresentados os campos da pressão e das velocidades nas direções $x$ e $z$, mostrando a evolução da superfície livre 3D. 

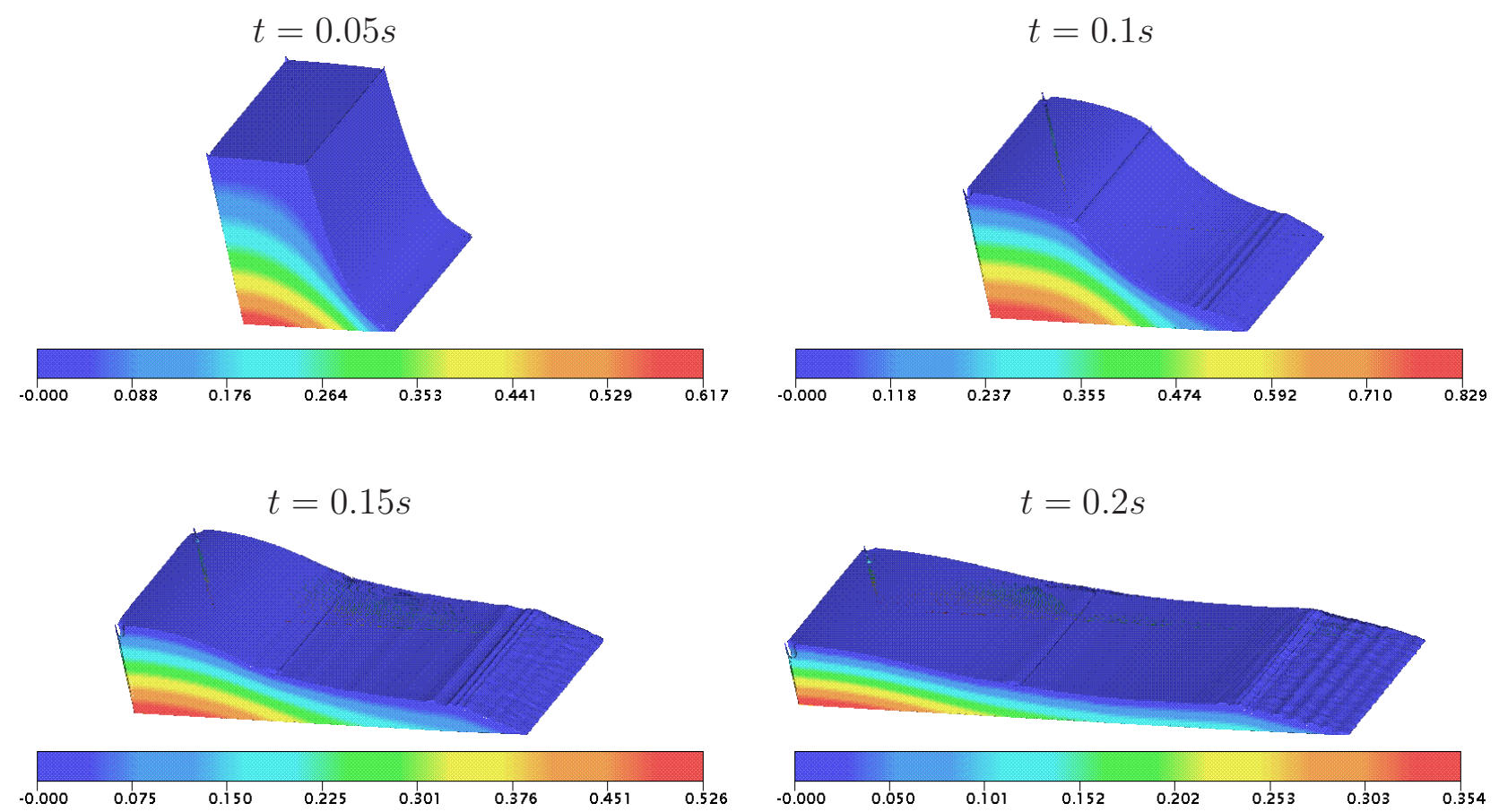

Figura 8.3: campo da pressão para o problema do colapso de um bloco de fluido em diferentes tempos.
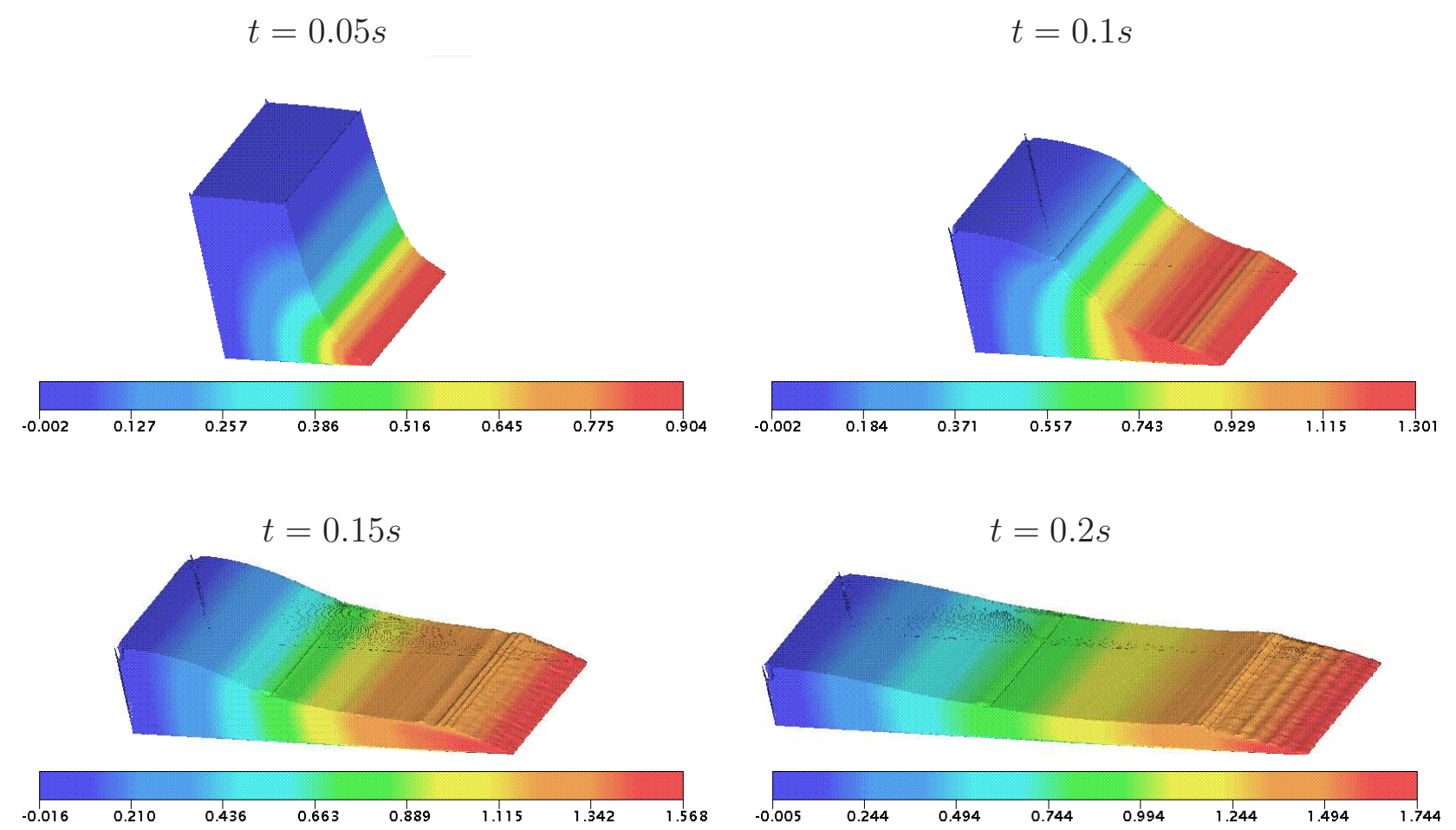

Figura 8.4: campo da velocidade na direção $x$ para o problema do colapso de um bloco de fluido em diferentes tempos. 

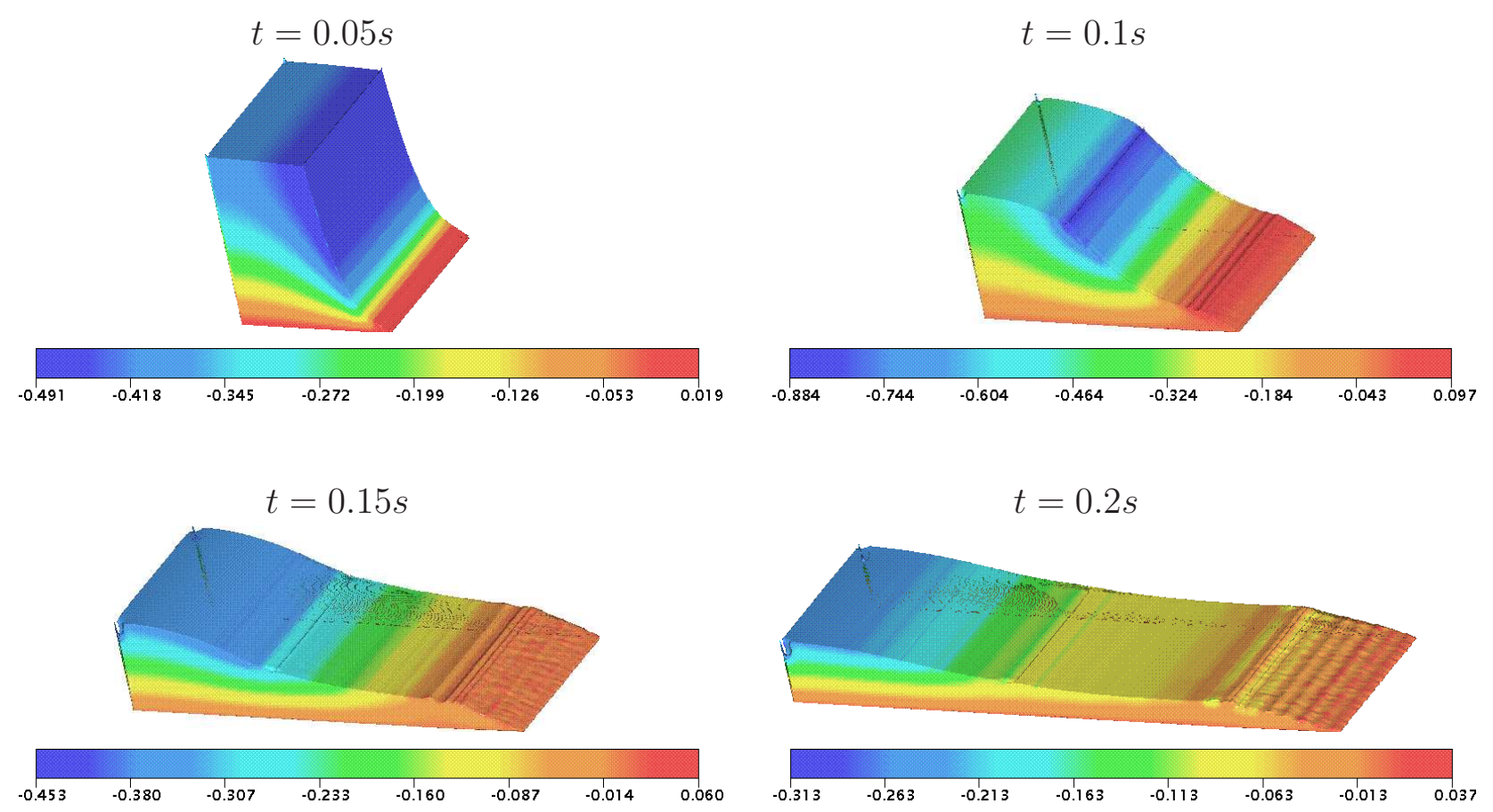

Figura 8.5: campo da velocidade na direção $z$ para o problema do colapso de um bloco de fluido em diferentes tempos.

\subsection{Ressalto Hidráulico Circular}

Nesta seção, o esquema EPUS é aplicado para simular o fenômeno salto hidráulico circular já discutido na Subseção 7.4.1. Para isso, dois testes diferentes são considerados.

Teste-2: o ressalto hidráulico circular é simulado aqui para o número de Reynolds $R e=250$, da mesma forma como já apresentado na Subseção 7.4.1 para o caso axissimétrico, só que agora usando as equações de Navier-Stokes 3D. A solução obtida também é comparada com o experimento apresentado em Rai [46]. Para a simulação, utilizam-se a condição de contorno no-slip e os seguintes dados:

- Malha: $100 \times 100 \times 35$ células computacionais $(\delta x=\delta y=\delta z=0.001)$;

- Domínio: $0.1 \times 0.1 \times 0.035$;

- Raio do injetor: $r_{i}=0.004 m$;

- Altura do injetor: $h_{i}=0.00075 m$;

- Escala de comprimento: $L_{0}=2 r_{i}=0.008 m$;

- Escala de velocidade (velocidade de injeção): $U_{0}=0.375 \mathrm{~m} / \mathrm{s}$;

- Coeficiente de viscosidade cinemática: $\nu=1.2 \cdot 10^{-5} \mathrm{~m}^{2} / \mathrm{s}$;

- Número de Reynolds: $R e=250$. 
Os resultados obtidos nesta simulação são apresentados na Figura 8.6, onde faz-se uma comparação com resultados experimentais. A partir desta figura, pode-se observar que, assim como no caso axissimétrico, o esquema EPUS simula com sucesso o fenômeno do ressalto hidráulico circular no caso tridimensional.

(a)

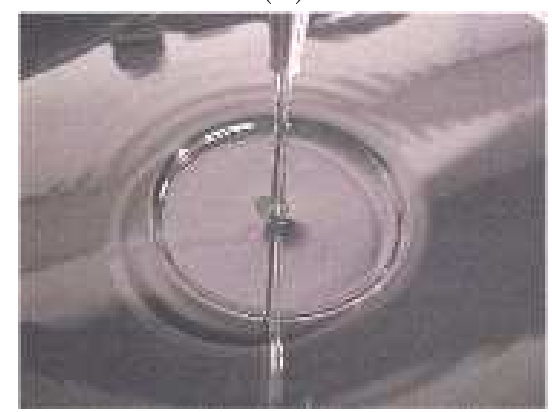

(b)

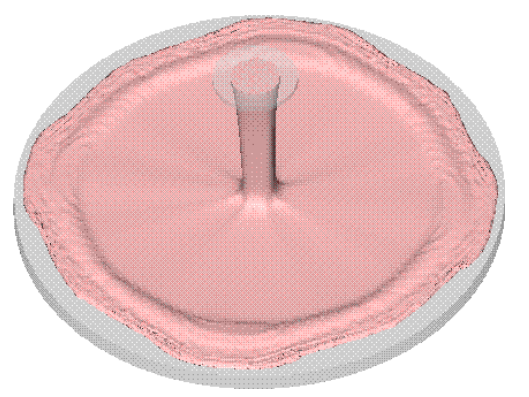

Figura 8.6: ilustração do ressalto hidráulico circular para $R e=250$ : resultados (a) experimental e (b) simulado pelo esquema EPUS.

Teste-3: neste teste o fenômeno do ressalto hidráulico circular é simulado para o número de Reynolds $R e=1000$, em que a formação de instabilidades físicas após o salto hidráulico é mais evidente que no Teste-2. A solução obtida pelo esquema EPUS é comparada aqui com o experimento de Ellegard [17]. Para isso, utilizam-se a condição de contorno no-slip e os seguintes dados:

- Malha: $120 \times 120 \times 10$ células computacionais $(\delta x=\delta y=\delta z=0.005)$;

- Domínio: $0.6 \times 0.6 \times 0.05$;

- Raio do injetor: $r_{i}=0.025 \mathrm{~m}$;

- Altura do injetor: $h_{i}=0.001 m$;

- Escala de comprimento: $L_{0}=2 r_{i}=0.005 m$;

- Escala de velocidade (velocidade de injeção): $U_{0}=1 \mathrm{~m} / \mathrm{s}$;

- Coeficiente de viscosidade cinemática: $\nu=5 \cdot 10^{-5} \mathrm{~m}^{2} / \mathrm{s}$;

- Número de Reynolds: $R e=1000$.

A Figura 8.7 mostra os resultados numéricos com o EPUS comparados com o experimento de Ellegard. A partir desta figura, observa-se que o esquema EPUS simula satisfatoriamente o fenômeno do ressalto hidráulico circular tridimensional para o caso de um alto valor do número de Reynolds. 
(a)

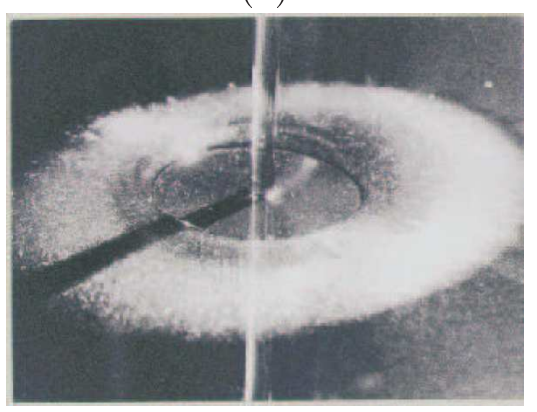

(b)

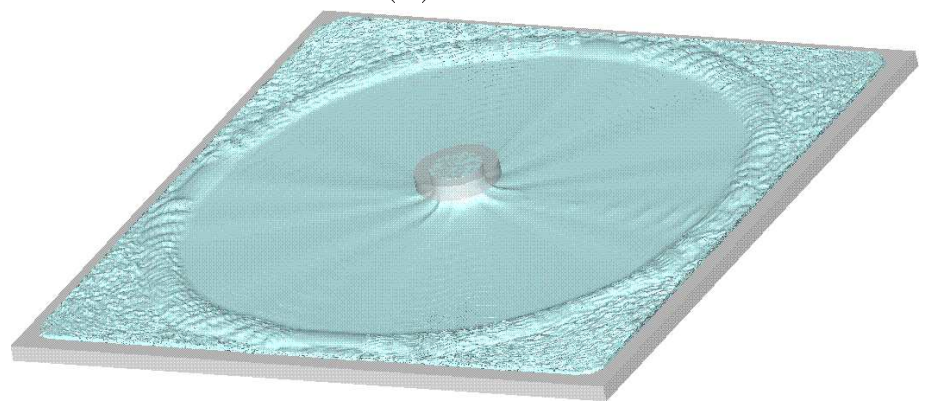

Figura 8.7: ilustração do ressalto hidráulico circular para $R e=1000$ : resultados (a) experimental e (b) simulado pelo esquema EPUS.

\subsection{Jato Circular Oscilante}

O fenômeno do jato circular oscilante é caracterizado pela formação de oscilações físicas (instabilidades) do fluido ao longo de seu escoamento (para mais detalhes, ver Cruickshank [14] e Ville [64]). Essas oscilações ocorrem quando um fluido altamente viscoso, e sob à ação da gravidade, incide perpendicularmente sobre uma superfície rígida impermeável (ver esquema Figura 8.8). Este fenômeno ocorre com muita frequência em aplicações industriais, como na moldagem por injeção de um propergol em cavidades com geometrias complexas. As instabilidades neste caso surgem durante a fase de enchimento do molde (ver, por exemplo, Ville [64]).

Nos últimos 40 anos, tem sido um grande desafio para os pesquisadores entender este tipo de escoamento (ver, por exemplo, [64]). A partir de diversos experimentos foi possível observar que as instabilidades ocorrem quando se obedecem determinadas relações de dois parâmetros críticos. Uma delas é a razão $H / d$ (comprimento da queda/diâmetro do injetor), a qual deve ser maior que o valor crítico $(H / d)_{c}=7.2$. A outra é o adimensional $R e$, que deve ser menor que o valor crítico $R e_{c}=1.2$ (para mais detalhes, ver Cruickshank [14]). É importante observar que, muito embora o esquema EPUS tenha sido planejado para simular problemas a altos Reynolds, ele também é útil para problemas a baixos Reynolds, como é mostrado a seguir.

Para a simulação deste fenômeno, consideram-se dois testes diferentes, sendo que para ambos adota-se condição de contorno no-slip.

Teste-4: neste teste, o problema do jato circular oscilante é simulado utilizando-se os seguintes dados:

- Malha: $50 \times 50 \times 63$ células computacionais $(\delta x=\delta y=\delta z=0.02)$;

- Domínio: $1 m \times 1 m \times 1.26 m$;

- Diâmetro do injetor: $d=0.08 m$;

- Altura do injetor: $h_{i}=0.2 m$; 


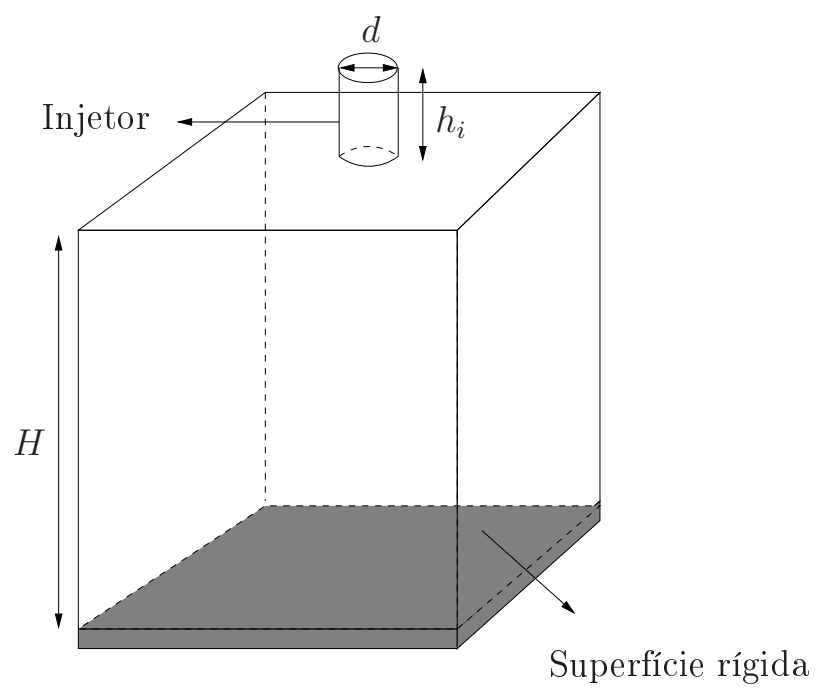

Figura 8.8: ilustração esquemática para problema do jato circular oscilante.

- Escala de comprimento: $L_{0}=d=0.08 m$;

- Escala de velocidade (velocidade de injeção): $U_{0}=1 \mathrm{~m} / \mathrm{s}$;

- Coeficiente de viscosidade cinemática: $\nu=0.278 \mathrm{~m}^{2} / \mathrm{s}$;

- Número de Reynolds: $R e=0.288$.

O valor da relação $H / d$ é 12.5. Este valor, juntamente com o número de Reynolds, permite verificar que as restrições para a formação das instabilidades (condições de Cruickshank) estão satisfeitas. A simulação do jato circular oscilante referente a este teste é apresentada na Figura 8.9, onde pode-se observar claramente que o esquema EPUS captura com sucesso o fenômeno.

Teste-5: neste teste, o caso Teste-4 é simulado novamente diminuindo-se pela metade o diâmetro do injetor. Para tanto, utilizam-se os seguintes dados:

- Malha: $100 \times 100 \times 123$ células computacionais $(\delta x=\delta y=\delta z=0.01)$;

- Domínio: $1 \mathrm{~m} \times 1 \mathrm{~m} \times 1.23 \mathrm{~m}$;

- Diâmetro do injetor: $d=0.04 m$;

- Altura do injetor: $h_{i}=0.2 m$;

- Escala de comprimento: $L_{0}=2 d=0.08 m$;

- Escala de velocidade (velocidade de injeção): $U_{0}=1 \mathrm{~m} / \mathrm{s}$;

- Coeficiente de viscosidade cinemática: $\nu=0.278 \mathrm{~m}^{2} / \mathrm{s}$;

- Número de Reynolds: $R e=0.288$.

A razão $H / d$ é 25 , a qual, juntamente com o número de Reynolds satisfazem as condições de Cruickshank. A simulação deste teste está ilustrada na Figura 8.10, onde compara-se a solução calculada com o EPUS com resultados experimentais (ver [40]). Mais uma vez, vê-se por essa 

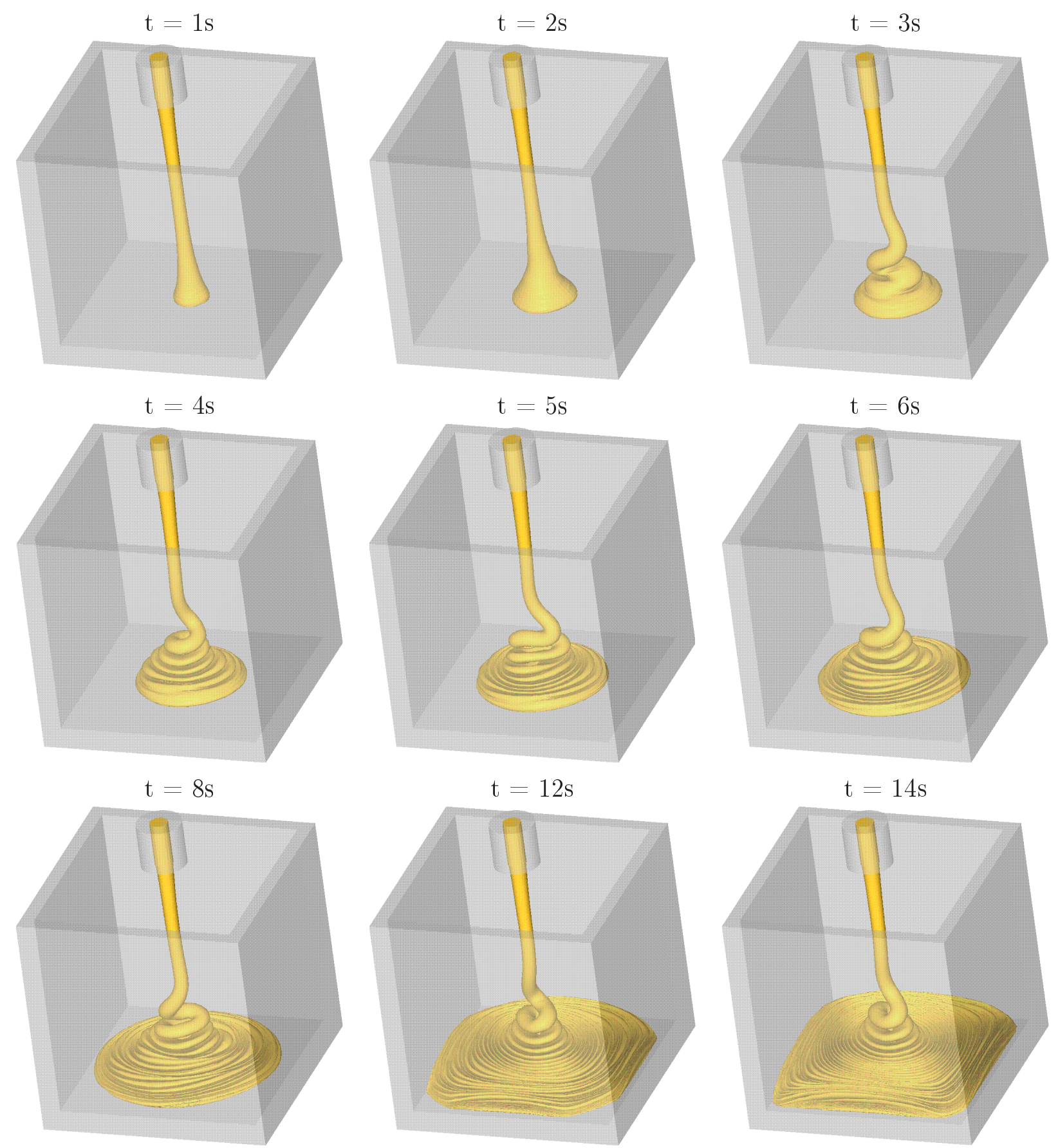

Figura 8.9: ilustração dos resultados obtidos pelo EPUS para o fenômeno do jato circular oscilante (Teste-4). 
comparação que, de fato, o esquema EPUS captura o fenômeno. Vale ressaltar ainda o fato de que, diminuindo-se o diâmetro do injetor (ou seja, aumentando a razão $H / d$ ), surgem algumas deficiências na formação das oscilações circulares, diferentemente dos resultados apresentados no Teste-4. 


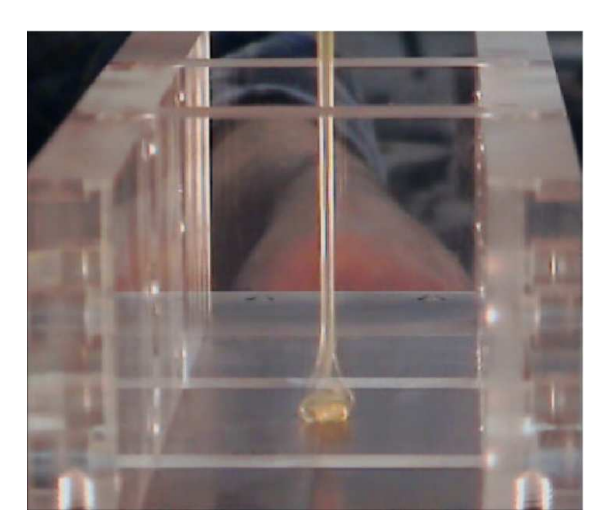

$\mathrm{t}=1.5 \mathrm{~s}$

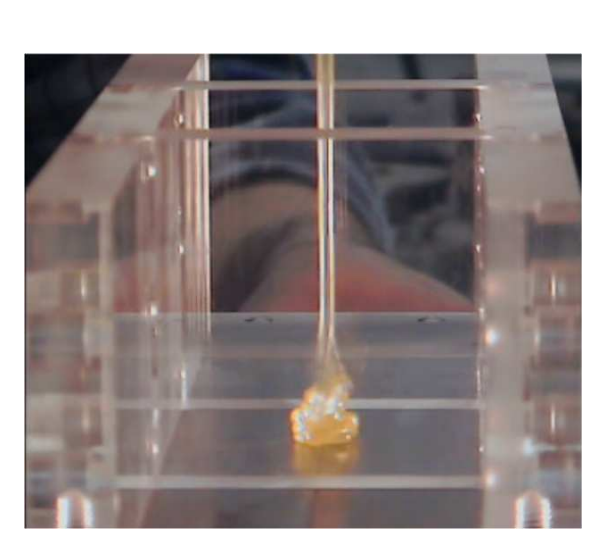

$\mathrm{t}=2.8 \mathrm{~s}$
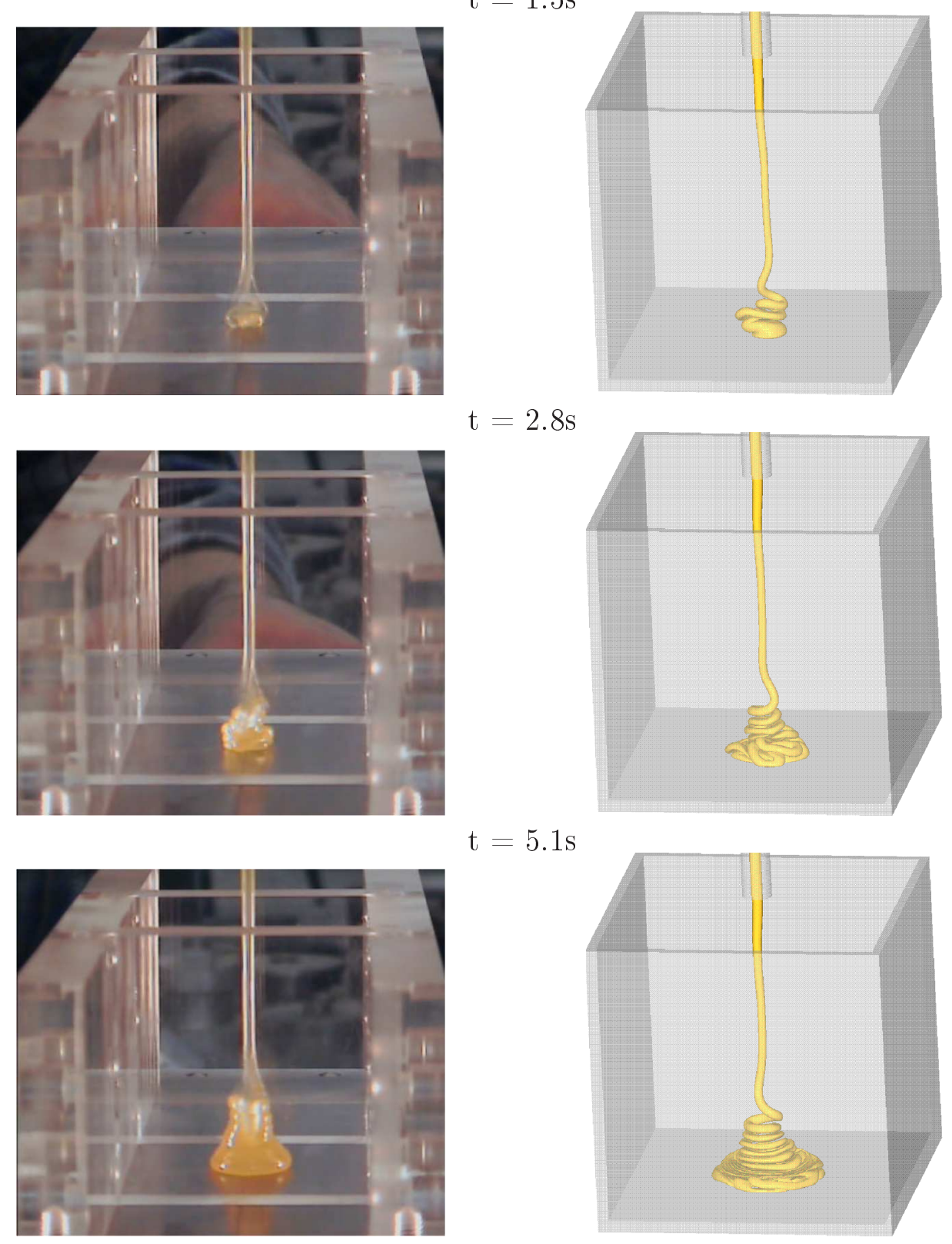

$\mathrm{t}=5.1 \mathrm{~s}$

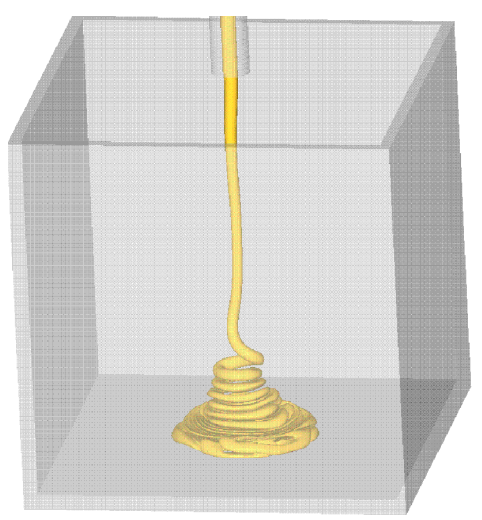

$\mathrm{t}=9.4 \mathrm{~s}$
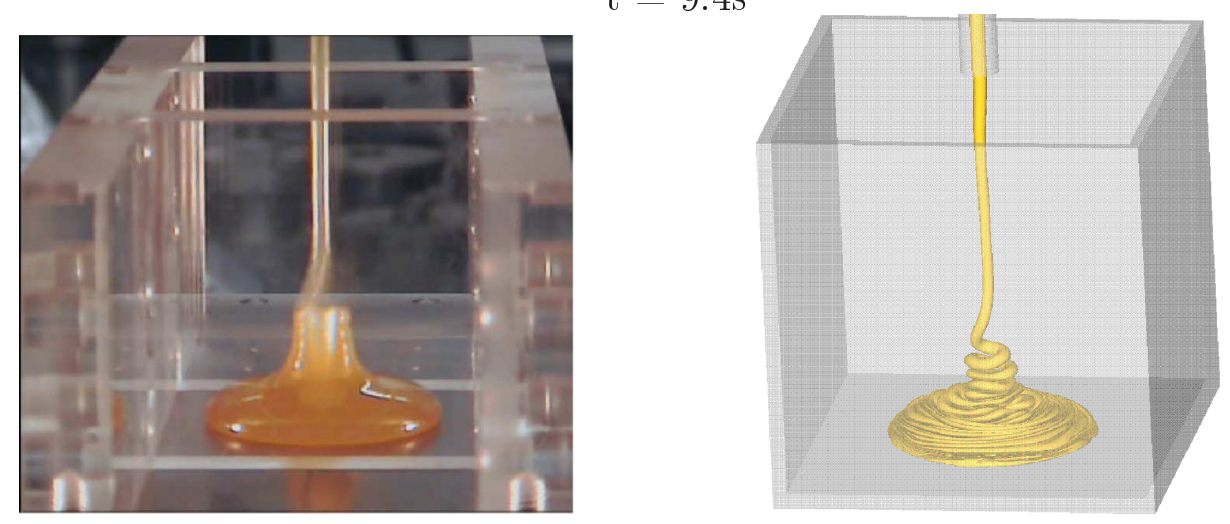

Figura 8.10: ilustração do jato circular oscilante para o Teste-5: resultados (a) experimental e (b) simulado com o esquema EPUS. 


\subsection{Jato Planar Oscilante}

Este problema apresenta características semelhantes ao jato circular oscilante. Para este caso, considera-se um injetor retangular (ver Figura 8.11), o qual proporciona a formação de dobras quando o fluido (altamente viscoso) atinge a superfície rígida (ver, por exemplo, [64]).

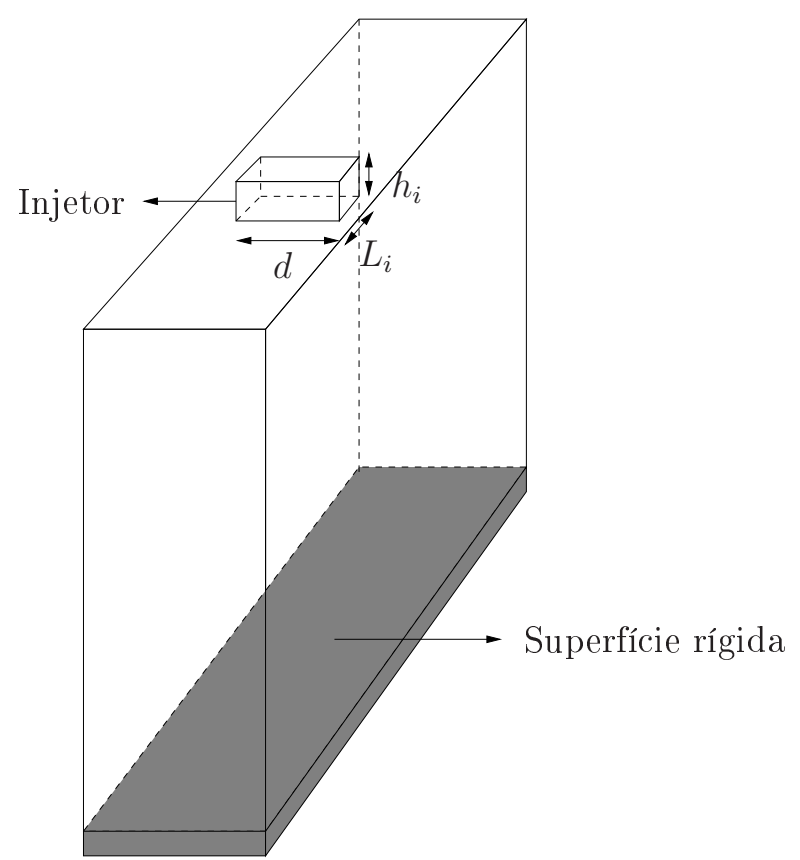

Figura 8.11: ilustração esquemática para o problema do jato planar oscilante.

Simula-se aqui o seguinte teste, com condição no-slip aplicada ao contorno rígido.

Teste-6: neste teste, o problema do jato planar oscilante é simulado com os seguintes dados:

- Malha: $146 \times 66 \times 213$ células computacionais $(\delta x=\delta y=\delta z=0.005)$;

- Domínio: $0.73 m \times 0.33 m \times 1.065 m$;

- Dimensões do injetor: $d=0.2 m, L_{i}=0.02 m$ e $h_{i}=0.05 m$;

- Escala de comprimento: $L_{0}=d=0.2 m$;

- Escala de velocidade: $U_{0}=1 \mathrm{~m} / \mathrm{s}$;

- Coeficiente de viscosidade cinemática: $\nu=0.278 \mathrm{~m}^{2} / \mathrm{s}$;

- Número de Reynolds: $R e=0.72$.

Os resultados obtidos nesta simulação são apresentados na Figura 8.12, a partir da qual pode-se inferir que o esquema EPUS captura com bom desempenho o fenômeno do jato planar oscilante, formando com sucesso as dobras que surgem quando o fluido toca a superfície rígida. 

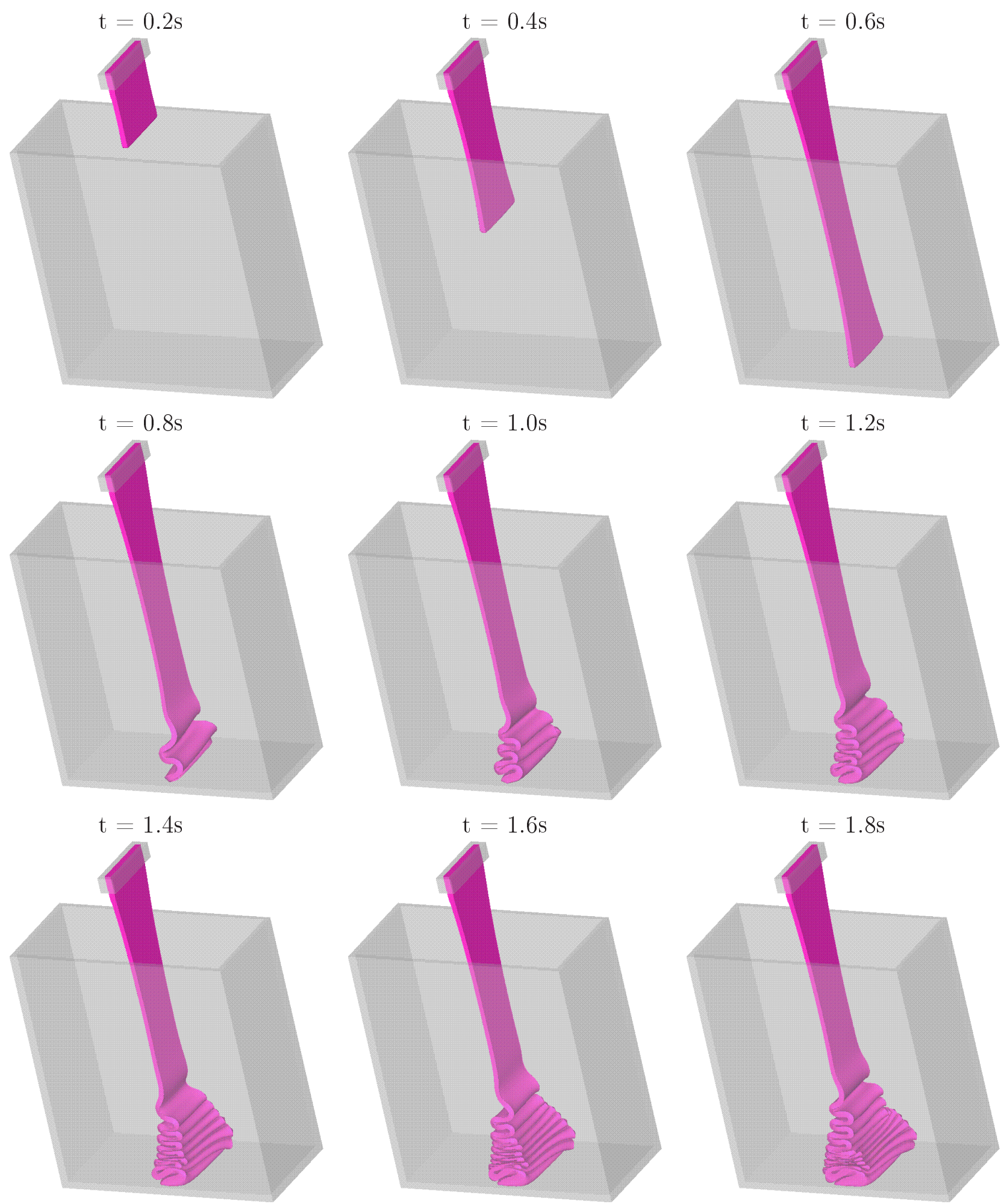

Figura 8.12: ilustração dos resultados obtidos pelo EPUS para o fenômeno do jato planar oscilante no Teste-6. 
CAPÍTULO 9

\section{Considerações Finais e Planos Futuros}

No presente trabalho de mestrado desenvolveu-se, analisou-se e implementou-se um novo esquema convectivo upwind de alta resolução para resolver leis de conservação e problemas de dinâmica dos fluidos relacionados. O novo esquema, então denominado EPUS, foi desenvolvido a partir de um polinômio de grau oito inserido nas regiões TVD/CBC. Ainda, este polinômio trata-se de uma função pertencente a classe de diferenciabilidade $C^{2}$ e satisfaz as condições de Leonard, ou seja, passa pelos $O(0,0)$ e $P(1,1)$ (para monotonicidade), pelo ponto $Q(0.5,0.75)$ (para atingir segunda ordem de precisão) e ainda passa pelo ponto $Q$ com inclinação 0.75 (para alcançar terceira ordem de precisão). Uma versão não oscilatória do esquema EPUS para simular problemas complexos foi introduzida definindo o parâmetro livre $\lambda=95$. Ademais, de acordo com o problema, o esquema EPUS pode também ser aplicado em outras situações segundo a variação de $\lambda$.

Com o objetivo de investigar o desempenho do esquema EPUS, ele foi aplicado na resolução de leis de conservação hiperbólicas, tais como as equações de advecção 1D, Burgers 1D, Euler 1D/2D e águas rasas 2D. E então, o EPUS foi aplicado para simular escoamentos incompressíveis não estacionários com superfícies livres móveis 2D (nos regimes laminar e turbulento), axissimétricos e 3D. Em particular, na simulação computacional das leis de conservação hiperbólicas foi utilizado o pacote computacional CLAWPACK ${ }^{1}$ desenvolvido por LeVeque (ver [32]) e para a simulação dos escoamentos incompressíveis não estacionários utilizou-se o código Freeflow de Castelo et al. [10].

Conforme apresentado nos capítulos de resultados numéricos (ver Capítulos 6, 7 e 8), o esquema EPUS teve bom desempenho nos testes numéricos propostos, mostrando ser uma ferramenta confiável na solução de leis de conservação e escoamentos incompressíveis não esta-

\footnotetext{
${ }^{1}$ http://www.amath.washington.edu/ claw/
} 
cionários com superfícies livres, e de fácil incorporação em pacotes computacionais disponíveis na literatura. Vale destacar que o esquema EPUS simulou com sucesso dois fenômenos físicos complexos: o salto hidráulico circular (no caso axissimétrico e tridimensional), o colapso de um bloco de fluido e as instabilidades físicas em jatos altamente viscosos (tridimensionais). Além disso, na simulação do salto hidráulico, os resultados obtidos com o esquema EPUS mostraram estar em boa concordância com a solução analítica de Watson dada pela Eq. (7.12) e com a estimativa para o raio do ressalto de Brechet e Néda dada pela Eq. (7.14).

Para o futuro, estão previstas as seguintes atividades de pesquisa:

- Dar continuidade a este trabalho de mestrado. Para tanto, pretende-se desenvolver uma metodologia numérica que combine os recentes avanços alcançados neste trabalho com novas ferramentas já existentes na área de CFD, permitindo a utilização de programas computacionais a um custo otimizado;

- Adaptar o esquema EPUS com o número de CFL. O objetivo aqui é melhorar a aproximação dos termos convectivos não lineares das equações de transporte e, consequentemente, conseguir métodos numéricos mais robustos, estáveis e com baixo custo computacional;

- Investigar o desempenho do esquema EPUS nas equações tridimensionais de Navier-Stokes para o regime turbulento;

- Utilizar a metodologia Simulação de Grandes Escalas (Large Eddy Simulation - LES) associada aos esquemas upwind desenvolvidos, a uma modelagem submalha (Subgrid Scale - SGS) e a uma relação algébrica para a tensão na parede (Wall Stress Model - WSM) - metodologia esta que tem adquirido popularidade crescente na literatura especializada (ver, por exemplo, [22]). O objetivo é incorporar mais física na modelagem global;

- Reunir os principais resultados obtidos neste trabalho em um artigo para revista internacional. 
CAPÍTULO

\section{Produção Científica Associada}

No decorrer deste trabalho de mestrado também voltou-se a atenção para a produção de artigos científicos, os quais seguem listados nas seções abaixo.

\subsection{Artigos e Resumos Publicados em Anais de Congres- SOS}

- Laís Corrêa, Magda Kimico Kaibara e Valdemir G. Ferreira, Leis de conservação: tratamento teórico e numérico, DINCON 2009 - 8th Brazilian Conference on Dynamics, Control and Applications, 2009, Bauru-SP.

Resumo: O principal objetivo deste artigo é a investigação das equações diferenciais parciais hiperbólicas tanto do ponto de vista teórico quanto numérico. Estas equações modelam problemas que envolvem conservação de propriedades físicas, o que é de grande interesse na ciência e na engenharia.

- Giseli A. B. Lima, Valdemir G. Ferreira, Rafael A. B. Queiroz, Miguel A. C. Candezano e Laís Corrêa, Development and evaluation of upwind schemes for conservation laws, COBEM2009 - International Congress of Mechanical Engineering, 2009, Gramado-RS.

Resumo: This work deals with a computational evaluation of two new high resolution upwind schemes, namely ADBQUICKEST (Int. J. Numer. Meth. Fluids 2009; 60:1-26) and TOPUS (Comput. Fluids - Submitted), for solving general hyperbolic conservation laws. By using the finite difference methodology, the schemes are analyzed and implemented in the context of normalized variables of Leonard (1988). In order to access the performance of these schemes, a series of one-dimensional test problems are examined beginning with classical linear advection of scalars and ending with Riemann problems for Burgers, Buckley-Leverett, shallow water, and Euler equations. And, as application, the ADBQUICK- 
EST and TOPUS schemes are used for the numerical simulation of $3 D$ incompressible Navier-Stokes equations involving free surface.

- Patrícia Sartori, Giseli A. B. Lima, Laís Corrêa, Miguel A.C. Candezano e Valdemir G. Ferreira, Avaliação computacional de três esquemas upwind originais, SIMMEC 2010 - $9^{\circ}$ Simpósio de Mecânica Computacional, 2010, São João Del Rei-MG.

Resumo: Resolver numericamente problemas em dinâmica dos fluidos é uma tarefa difícil e desafiadora, principalmente quando tais problemas são dominados por convecção. Isso requer o desenvolvimento de esquemas numéricos tipo upwind que sejam precisos, monotônicos e robustos. O presente trabalho é destinado à avaliação computacional de três novos esquemas upwind de alta resolução, desenvolvidos no LCAD-ICMC/USP, denominados ADBQUICKEST (ADaptative Quickest), TOPUS (Third-Order Polynomial Upwind Scheme) e SDPUS-C1 (Six-Degree Polynomial Upwind Scheme of C1 Class). O desempenho desses esquemas é investigado a partir da simulação computacional de problemas de leis de conservação hiperbólicas, a saber, advecção e problemas de Riemann para acústica e Euler da dinâmica dos gases. E então, como aplicação, esses esquemas são utilizados na simulação de escoamentos incompressíveis com superfícies livres móveis modelados pelas equações de Navier-Stokes $3 \mathrm{D}$.

- Laís Corrêa, Giseli A. B. Lima, Valdemir G. Ferreira, Solving fluid dynamics problems using a new polynomial upwind convection scheme, DINCON 2010 - 9th Brazilian Conference on Dynamics, Control and Their Applications, 2010, Serra Negra-SP.

Resumo: The purpose of this work is to present a new polynomial convection scheme for solving complex fluid dynamics problems. The scheme is evaluated in linear and nonlinear hyperbolic conservation laws and then is applied for simulating axisymmetric flow with moving free surfaces. From the results, the scheme shows to be a good tool for CFD.

- Giseli A. B. Lima, Laís Corrêa, Valdemir G. Ferreira, The new FDPUS-C1 scheme, 2010, Serra Negra-SP.

Resumo: In this work, we present a new high resolution polynomial upwind scheme, namely FDPUS-C1. Numerical results are presented for 1D/2D hyperbolic conservation laws. Then, as an application, the FDPUS-C1 scheme is used for solving of $2 D$ incompressible fluid that involves moving free surfaces. The numerical results show that the new convective upwind scheme behaves well under several CFL.

- Giseli A. B. Lima, Laís Corrêa, Miguel A.C. Candezano, Patrícia Sartori e Valdemir G. Ferreira, A simple NVD/TVD-based upwinding scheme for convection term discretization, ECCOMAS CFD 2010 - Fifth European Conference on Computational Fluid Dynamics, 2010, Lisboa-Portugal.

Resumo: The correct modeling for processes involving convection, without introducing excessive artificial damping while retaining high accuracy, stability, boundedness and simplicity of implementation continues being nowadays a challenging task for the cientific CFD community. In this context, the 
objective of this study is to present and to evaluate the performance of a new TVD-based upwinding scheme, namely Six Degree Polynomial Upwind Scheme of C1 Class (SDPUS-C1), for convection term discretization. SDPUS-C1 satisfies the TVD principle of Harten and is based on the NVD formulation of Leonard. Firstly, a description of the scheme is done and then numerical results are presented for three-dimensional hyperbolic conservation laws, such as acoustics, Burgers and Euler equations. Finally, as application, the SDPUS-C1 scheme is used for the computational simulation of three-dimensional incompressible fluid flows involving moving free surfaces.

- Giseli A. B. Lima, Laís Corrêa, Miguel A.C. Candezano, Patrícia Sartori e Valdemir G. Ferreira, Two upwinding schemes for nonlinear problems in fluid dynamics, Dynamics Days South America-Internacional Conference on Chaos and Nonlinear Dynamics, 2010, São José dos Campos-SP.

Resumo: The appropriated modeling of convection terms is a key point for reproducing complex physical phenomena in fluid dynamics problems, particularly in hyperbolic nonlinear conservation laws and related fluid flow problems. In this context, the objective of this work is to present/compare two new high-order upwind schemes for approximating convective terms, namely the Six-Degree Polynomial Upwind Scheme of C1 Class (SDPUS-C1) and the "Esquema teste" scheme. "Esquema teste" has the same properties of the SDPUS-C1 scheme, but it is of C2 class. These schemes are based on normalized variable diagram of Leonard and the TVD principle of Harten.

- Laís Corrêa, Giseli A. B. Lima, Patrícia Sartori, Miguel A.C. Candezano e Valdemir G. Ferreira, A new polynomial upwind convection scheme for fluid flow simulations, CONEM 2010 - VI Congresso Nacional de Engenharia Mecânica, 2010, Campina Grande-PB.

Resumo: The simulation of fluid flow problems involving strong convective character is a difficult problem to solve and has atracted many researchers in the CFD community. In this scenario, we present in this work a new polynomial upwind scheme, called SDPUS ("Six Degree Polynomial Upwind Scheme of C1 Class"), for numerical solution of conservation laws and related fluid dynamics problems. The scheme is developed in the context of normalized variables of Leonard and satisfies the CBC and TVD stability criteria of Gaskell and Lau, and Harten, respectively. The numerical solutions obtained with this scheme can achieve second/third order of accuracy in smooth regions and first order near to discontinuities (shocks). The performance of the SDPUS is assessed in the solution of nonlinear hyperbolic systems, such as shallow water, acoustics, and Euler equations of gas dynamics. As application, the scheme is then used in the solution of incompressible Navier-Stokes equations in cylindrical coordinates. From numerical results, one can clearly see that the SDPUS-C1 scheme is a robust tool for resolving both compressible and incompressible complex flow problems.

- Laís Corrêa, Giseli A. B. Lima, Valdemir G. Ferreira, A $C^{2}$ class-based upwinding scheme for aproximating terms, CNMAC 2010 - XXXIII Congresso Nacional de Matemática Aplicada e Computacional, 2010, Águas de Lindóia-SP. 
Resumo: Numerical difficulties are frequent when one intends to approximate convective terms (in general nonlinear) of conservation laws and related fluid dynamics problems. In this scenario, we present in this work a $C^{2}$ class TVD-based upwinding scheme for approximating convection terms. In a more general way as was made by Lin and Chieng [2], this differenciability property is imposed to avoid convergence problems when coarse meshes are employed.

- Giseli A. B. Lima, Laís Corrêa, Valdemir G. Ferreira, Uma nova classe de esquemas convectivos, V Encontro dos Pós-graduandos do IMECC, 2010, Campinas-SP.

Resumo: A aproximação numérica correta dos modelos matemáticos convectivos continua sendo uma tarefa desafiadora para a comunidade científica de CFD (Computational Fluid Dynamics). Essa aproximação numérica correta requer boa precisão, estabilidade, limitação e simplicidade de execução. Neste contexto, os objetivos desta pesquisa científica são apresentar e avaliar o desempenho de uma nova classe de esquemas upwinding polinomiais de alta precisão, para a discretização de termos convectivos, denominada SDPUS-C1 (Six-Degree Polynomial Upwind Scheme of $C^{1}$ Class). Essa classe de esquemas é baseada na formulação NVD (Normalized Variable Diagram) de Leonard e satisfaz as restrições TVD (Total Variation Diminishing) de Harten. Para alcançar esses objetivos, em primeiro lugar será feita uma descrição do desenvolvimento da classe de esquemas SDPUS-C1. Em seguida, apresenta-se o esquema representante dessa classe através de análise de alguns resultados numéricos obtidos para advecção de escalares. Logo depois, esse esquema é aplicado na resolução de leis de conservação 2D tais como acústica e águas rasas. Finalmente, como aplicação, o esquema SDPUS-C1 é usado para a simulação computacional de escoamentos incompressíveis bidimensionais e tridimensionais envolvendo superfícies livres móveis à alto números de Reynolds.

- Miguel A. C. Candezano, Laís Corrêa, Giseli A. B. Lima, Patrícia sartori, Valdemir G. Ferreira, A computational evaluation of two new high-resolution convective schemes for fluid dynamics problems, ENCIT 2010 - 13th Brazilian Congress of Thermal Scences and Engineering, 2010, Uberlândia-MG.

Resumo: It is well recognized that researchers face many problems for numerically approximating nonlinear convective terms in conservation laws and related fluid dynamics problems. One of the main challenges is to develop upwind schemes that capture well discontinuities (or shock waves) and allow high (at least second order) accuracy solution. In this scenario, the goal of this work is to present a computational evaluation of two genuinely Brazilian high resolution convective upwind schemes, namely ADBQUICKEST and SDPUS-C1, for solving general fluid dynamics problems. Both schemes are developed in the context of normalized variables (NV) of Leonard and satisfy the total-variation diminishing (TVD) constraints of Harten. 


\subsection{Artigos Submetidos em Anais de Congressos}

- Laís Corrêa, Giseli A. B. Lima, Valdemir G. Ferreira, Simulating compressible and incompressible flows using a new high resolution upwind convection scheme, submetido ao COBEM 2011 - International Congress of Mechanical Engineering, Natal-RN.

Resumo: Convection schemes of high resolution are extensively used nowadays to solve fluid dynamics problems, especially the incompressible class of flows involving high values of Reynolds number with moving free surfaces. Numerical solutions for this class of problems are difficult to find, because of the strong influence of nonlinear convective terms in the transport equations. Consequently, the choice of the numerical method that takes into account the flow direction (upwinding) has atracted many researchers in the modern CFD community. In this sense and with these motivations, we present in this work a new high resolution polynomial upwind convection scheme, called EPUS (Eight-degree Polynomial Upwind Scheme), for the numerical solution of systems of conservation laws and related fluid dynamics problems. The new scheme is developed by using a polynomial of eight-degree in the context of normalized variables of Leonard, that satisfies the CBC (Convection Boundedness Criterion) and TVD (Total Variation Diminishing) stability criteria. An important property of the high resolution EPUS scheme is to be as accurate as possible in smooth regions and with controled numerical dissipation in regions of high gradients and discontinuites. The performance of the EPUS scheme is assessed in the numerical solution of compressible Euler equations of the gas dynamics. As application, the scheme is then used for solving incompressible Navier-Stokes equations; in particular, the numerical solutions of the circular hydraulic jump and broken-dam problems are presented. The numerical results confirm that the EPUS scheme is a effective tool for resolving both compressible and incompressible complex flow problems.

\subsection{Artigos Aceitos em Revistas Internacionais}

- Giseli A. B. Lima, Laís Corrêa, Valdemir G. Ferreira, Two upwinding schemes for nonlinear problems in fluid dynamics, Journal of Phisics: Conference Series.

Resumo: The correct modeling for processes involving convection, without introducing excessive artificial damping while retaining high accuracy, stability, boundedness and simplicity of implementation continues being nowadays a challenging task for CFD practitioners. The objective of this study is to present and evaluate the performance of two new upwinding schemes, namely SDPUS-C1 and EPUS, for nonlinear convection term discretization. Both SDPUS-C1 and EPUS schemes satisfy the TVD principle of Harten and are based on the NVD formulation of Leonard. Firstly, a description of the schemes is presented and then the numerical results are provided for one- and two-dimensional hyperbolic conservation laws. Finally, as an application, the SDPUS-C1 and EPUS schemes are employed for the simulation of two-dimensional incompressible fluid flows involving moving free surfaces. The numerical experiments show that the proposed upwinding schemes perform very well. 


\subsection{Cursos de Curta Duração Ministrados}

- Giseli A. B. Lima, Laís Corrêa, Valdemir G. Ferreira, Simulação computacional de alguns problemas em dinâmica dos fluidos, DINCON 2010 - 9th Brazilian Conference on Dynamics, Control and Their Applications, 2010, Serra Negra-SP.

Resumo: O presente minicurso é direcionado, principalmente, a alunos de graduação que tenham alguma familiaridade com cálculo avançado, cálculo numérico e física elementar. A proposta do minicurso é mostrar que dinâmica dos fluidos computacional é a ciência de construir soluções numéricas para equações de conservação, avançando a solução no espaço e no tempo para obter uma descrição numérica do escoamento de interesse. O seu objetivo principal é mostrar ao aluno como resolver, no contexto de diferenças finitas, as equações de Navier-Stokes para o caso incompressível. 


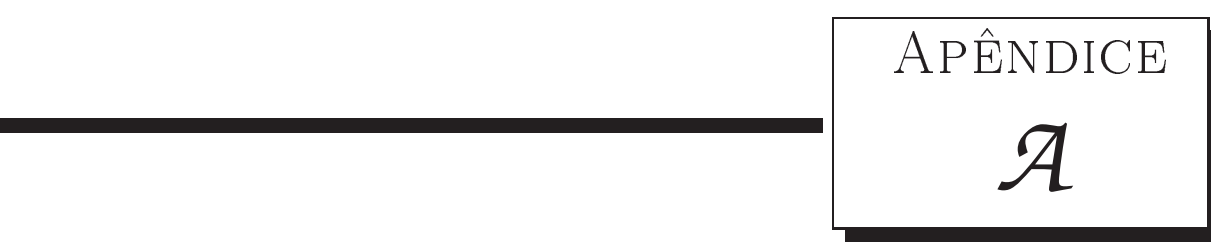

EPUS e a Propriedade TVD

Como visto no Capítulo 4, o esquema EPUS é dependente de um parâmetro livre $\lambda$. No entanto, como uma das principais propostas do novo esquema é que ele satisfaça a restrição TVD de estabilidade, deve-se determinar para quais parâmetros o novo esquema satisfaz essa propriedade. O intervalo de $\lambda$ para o qual o EPUS é TVD foi apresentado no Capítulo 4 e é dado por $16 \leq \lambda \leq 95$. Neste apêndice demonstra-se como este intervalo foi obtido.

Considere o esquema EPUS escrito em variáveis normalizadas:

$$
\hat{\phi}_{f}=\left\{\begin{array}{rrr}
-4(\lambda-24) \hat{\phi}_{U}^{8}+16(\lambda-23) \hat{\phi}_{U}^{7}+(528-25 \lambda) \hat{\phi}_{U}^{6}+ & \\
+(19 \lambda-336) \hat{\phi}_{U}^{5}+(80-7 \lambda) \hat{\phi}_{U}^{4}+\lambda \hat{\phi}_{U}^{3}+\hat{\phi}_{U}, & \text { se } \hat{\phi}_{U} \in[0,1], \\
\hat{\phi}_{U}, & \text { se } \quad \hat{\phi}_{U} \notin[0,1] .
\end{array}\right.
$$

Na seção 3.5 apresenta-se a seguinte relação entre variáveis normalizadas e a restrição TVD:

$$
\begin{cases}\hat{\phi}_{U} \leq \hat{\phi}_{f} \leq \min \left\{1,2 \hat{\phi}_{U}\right\}, & \text { se } \quad \hat{\phi}_{U} \in[0,1], \\ \hat{\phi}_{f}=\hat{\phi}_{U}, & \text { se } \quad \hat{\phi}_{U} \notin[0,1],\end{cases}
$$

em que dado um esquema em variáveis normalizadas, se a restrição acima for satisfeita, então tem-se um esquema TVD. A partir destas relações, demonstra-se a seguir que de fato para os valores de $\lambda \in[16,95]$ tem-se que o esquema EPUS é TVD.

\section{Demonstração:}

Claramente observa-se que a segunda condicional de (A.2) é diretamente satisfeita pela segunda condicional de (A.1), em que $\hat{\phi}_{f}=\hat{\phi}_{U}$ para $\hat{\phi}_{U} \notin[0,1]$.

Com isso, falta demonstrar a primeira condicional de (A.2). Para isso, demonstra-se as três seguintes condições: 
(1) $\hat{\phi}_{f} \geq \hat{\phi}_{U}$ para $\hat{\phi}_{U} \in[0,1]:$

Considere $r_{1}=\hat{\phi}_{f}-\hat{\phi}_{U}$. Substituindo a primeira condicional de (A.1) em $r_{1}$, tem-se

$$
r_{1}=-4(\lambda-24) \hat{\phi}_{U}^{8}+16(\lambda-23) \hat{\phi}_{U}^{7}+(528-25 \lambda) \hat{\phi}_{U}^{6}+(19 \lambda-336) \hat{\phi}_{U}^{5}+(80-7 \lambda) \hat{\phi}_{U}^{4}+\lambda \hat{\phi}_{U}^{3} .
$$

Com isso, tem-se que mostrar que $r_{1} \geq 0$ para $\hat{\phi}_{U} \in[0,1]$. Para calcular quais parâmetros $\lambda$ satisfazem essa condição, utilizou-se um programa em linguagem C, o qual varre valores inteiros de $\lambda$ de 0 a 1000. A partir deste programa, obteve-se que os valores para os quais $r_{1} \geq 0$ são $\lambda \in[16,1000]$.

(2) $\hat{\phi}_{f} \leq 2 \hat{\phi}_{U}$ para $\hat{\phi}_{U} \in[0,0.5]$ :

Considere $r_{2}=\hat{\phi}_{f}-2 \hat{\phi}_{U}$. Substituindo a primeira condicional de (A.1) em $r_{2}$, tem-se

$$
r_{2}=-4(\lambda-24) \hat{\phi}_{U}^{8}+16(\lambda-23) \hat{\phi}_{U}^{7}+(528-25 \lambda) \hat{\phi}_{U}^{6}+(19 \lambda-336) \hat{\phi}_{U}^{5}+(80-7 \lambda) \hat{\phi}_{U}^{4}+\lambda \hat{\phi}_{U}^{3}-\hat{\phi}_{U} .
$$

Com isso, tem-se que mostrar que $r_{2} \leq 0$ para $\hat{\phi}_{U} \in[0,0.5]$. Para calcular quais parâmetros $\lambda$ satisfazem essa condição, utilizou-se o mesmo programa do caso (1), a partir do qual obtiveram-se que os valores para os quais $r_{2} \leq 0$ são $\lambda \in[0,95]$.

(3) $\hat{\phi}_{f} \leq 1$ para $\hat{\phi}_{U} \in[0.5,1]:$

Considere $r_{3}=\hat{\phi}_{f}-1$. Substituindo a primeira condicional de (A.1) em $r_{3}$, tem-se $r_{3}=-4(\lambda-24) \hat{\phi}_{U}^{8}+16(\lambda-23) \hat{\phi}_{U}^{7}+(528-25 \lambda) \hat{\phi}_{U}^{6}+(19 \lambda-336) \hat{\phi}_{U}^{5}+(80-7 \lambda) \hat{\phi}_{U}^{4}+\lambda \hat{\phi}_{U}^{3}+\hat{\phi}_{U}-1$.

Com isso, tem-se que mostrar que $r_{3} \leq 0$ para $\hat{\phi}_{U} \in[0.5,1]$. Utilizando o mesmo programa dos casos anteriores para varrer os valores de $\lambda$ obteve-se que os valores para os quais $r_{3} \leq 0$ são $\lambda \in[0,1000]$.

Para obter o intervalo final de valores de $\lambda$ para os quais o esquema EPUS é TVD, faz-se a interseção dos 3 intervalos obtidos nos casos (1), (2) e (3), resultando em $\lambda \in[16,95]$. Com isso, conforme visto ao longo deste texto, apenas valores dentro deste intervalo foram utilizados para aplicar o esquema EPUS, já que um dos objetivos de desenvolver um novo método é a proposta de que este seja TVD. 
APÊNDICE $\mathcal{B}$

\section{Esquema Desenvolvido Sem Parâmetro Livre}

Ao longo deste trabalho, desenvolveu-se também um outro esquema com as mesmas propriedades do esquema EPUS, no entanto sem a presença de um parâmetro livre. Este novo esquema foi testado em várias leis de conservação 1D e 2D, no entanto, como o esquema EPUS apresentou melhores resultados em todos estes testes, o esquema sem parâmetro livre foi desconsiderado como nova proposta neste trabalho, sendo apresentado apenas como apêndice.

No desenvolvimento do esquema sem parâmetro livre foram impostas oito condições para sua derivação. Sendo assim, para o desenvolvimento do esquema considerou-se um polinômio de grau sete, da forma

$$
\hat{\phi}_{f}\left(\hat{\phi}_{U}\right)=\left\{\begin{array}{lll}
a_{7} \hat{\phi}_{U}^{7}+a_{6} \hat{\phi}_{U}^{6}+a_{5} \hat{\phi}_{U}^{5}+a_{4} \hat{\phi}_{U}^{4}+a_{3} \hat{\phi}_{U}^{3}+a_{2} \hat{\phi}_{U}^{2}+a_{1} \hat{\phi}_{U}+a_{0}, & \text { se } \hat{\phi}_{U} \in[0,1], \\
\hat{\phi}_{U}, & \text { se } \quad \hat{\phi}_{U} \notin[0,1] .
\end{array}\right.
$$

Na determinação dos coeficientes $a_{0}, \ldots, a_{7}$ foram impostas, primeiramente, as condições de Leonard, como segue:

- passar pelo ponto $O(0,0)$;

- passar pelo ponto $P(1,1)$;

- passar pelo ponto $Q(0.5,0.75)$ (condição necessária e suficiente para atingir segunda ordem de precisão);

- ter inclinação de 0.75 no ponto $Q$ (condição necessária e suficiente para alcançar terceira ordem).

Aplicando tais condições em (B.1), tem-se

$-\hat{\phi}_{f}(0)=0$ :

$$
a_{0}=0 ;
$$


$-\hat{\phi}_{f}(1)=1$ :

$$
a_{7}+a_{6}+a_{5}+a_{4}+a_{3}+a_{2}+a_{1}=1
$$

$-\hat{\phi}_{f}(0.5)=0.75$

$$
a_{7}+2 a_{6}+4 a_{5}+8 a_{4}+16 a_{3}+32 a_{2}+64 a_{1}=96
$$

$-\hat{\phi}_{f}^{\prime}(0.5)=0.75$

$$
7 a_{7}+12 a_{6}+20 a_{5}+32 a_{4}+48 a_{3}+64 a_{2}+64 a_{1}=48 .
$$

Em adição a isso, para fechar o sistema, considerou-se que o polinômio de grau sete seja uma função de classe $C^{2}$, assim como feito para o esquema EPUS.

Para isso, as seguintes condições devem ser satisfeitas, sendo que as duas primeiras determinam que ele seja de classe $C^{1}$ e as duas últimas que se estenda para classe $C^{2}$, as quais seguem aplicadas em (B.1):

$-\hat{\phi}_{f}^{\prime}(0)=1$ :

$$
a_{1}=1
$$

$-\hat{\phi}_{f}^{\prime}(1)=1:$

$$
7 a_{7}+6 a_{6}+5 a_{5}+4 a_{4}+3 a_{3}+2 a_{2}+a_{1}=1
$$

$-\hat{\phi}_{f}^{\prime \prime}(0)=0$ :

$$
a_{2}=0
$$

$-\hat{\phi}_{f}^{\prime \prime}(1)=0$ :

$$
42 a_{7}+30 a_{6}+20 a_{5}+12 a_{4}+6 a_{3}+2 a_{2}=0 .
$$

Com a resolução do sistema formado por (B.2)-(B.9), obtém-se

$$
a_{7}=16 ; a_{6}=-72 ; a_{5}=120 ; a_{4}=-88 ; a_{3}=24 ; a_{2}=0 ; a_{1}=1 ; a_{0}=0 \text {. }
$$

Finalmente, o esquema sem parâmetro livre, em variáveis normalizadas, é dado por

$$
\hat{\phi}_{f}=\left\{\begin{array}{lll}
16 \hat{\phi}_{U}^{7}-72 \hat{\phi}_{U}^{6}+120 \hat{\phi}_{U}^{5}-88 \hat{\phi}_{U}^{4}+24 \hat{\phi}_{U}^{3}+\hat{\phi}_{U}, & \text { se } & \hat{\phi}_{U} \in[0,1] \\
\hat{\phi}_{U}, & \text { se } & \hat{\phi}_{U} \notin[0,1]
\end{array}\right.
$$


Utilizando a definição de variáveis normalizadas (NV), tem-se a equação do esquema sem parâmetro livre em variáveis não-normalizadas:

$$
\phi_{f}=\left\{\begin{array}{lll}
\phi_{R}+\left(\phi_{D}-\phi_{R}\right)\left[16 \hat{\phi}_{U}^{7}-72 \hat{\phi}_{U}^{6}+120 \hat{\phi}_{U}^{5}-88 \hat{\phi}_{U}^{4}+24 \hat{\phi}_{U}^{3}+\hat{\phi}_{U}\right], & \text { se } \hat{\phi}_{U} \in[0,1] \\
\phi_{U}, & \text { se } \hat{\phi}_{U} \notin[0,1]
\end{array}\right.
$$

Ainda, pode-se reescrever o esquema na forma de limitador de fluxo, o qual é dado por

$$
\psi\left(r_{f}\right)= \begin{cases}\frac{16 r_{f}^{4}+48 r_{f}^{3}}{\left(1+r_{f}\right)^{6}}, & \text { se } r_{f} \geq 0 \\ 0, & \text { se } r_{f}<0\end{cases}
$$


APÊNDICE

\section{Software CLAWPACK}

Para resolver as leis de conservação 1D dadas pela Eq. (2.1), consideram-se estas equações escritas na forma quase-linear, a qual é dada por

$$
\phi_{t}+A(x, t) \phi_{x}=0
$$

em que $A(x, t)$ é a matriz jacobiana $F^{\prime}(\phi(x, t))=A(x, t)$.

Para resolver (C.1) é empregado o algoritmo a seguir, o qual é conhecido como algoritmo REA (ver LeVeque [32]):

Passo 1: Substitui-se a solução pontual por uma distribuição constante por partes;

Passo 2: Resolve-se o problema de Riemann aproximado pelo método de Roe. O solucionador do problema de Riemann retorna, para quaisquer dois estados $\phi_{i-1}$ e $\phi_{i}$, um conjunto de $M$ ondas definidas como

$$
\sum_{q=1}^{M} \mathcal{W}_{i-\frac{1}{2}}^{q}=\phi_{i}-\phi_{i-\frac{1}{2}}=\Delta \phi_{i-\frac{1}{2}}
$$

e as flutuações

$$
\begin{aligned}
& \mathcal{A}^{-\Delta} \phi_{i-\frac{1}{2}}=\sum_{q}^{M}\left(\hat{s}^{q}\right)^{-} \mathcal{W}_{i-\frac{1}{2}}^{q}, \\
& \mathcal{A}^{+} \Delta \phi_{i-\frac{1}{2}}=\sum_{q}^{M}\left(\hat{s}^{q}\right)^{+} \mathcal{W}_{i-\frac{1}{2}}^{q},
\end{aligned}
$$

onde $\left(\hat{s}^{q}\right)^{-}=\min (\hat{\lambda}, 0)$ e $\left(\hat{s}^{q}\right)^{+}=\max (\hat{\lambda}, 0)$, em que $\hat{\lambda}$ é o autovalor da matriz $A$.

Passo 3: Aplica-se a fórmula de atualização, as quais são dadas utilizando-se o método de 
Godunov de primeira ordem ou sua variante de segunda ordem com termo de correção (proposta por LeVeque [32]):

- método de primeira ordem de Godunov:

$$
\phi_{i}^{n+1}=\phi_{i}^{n}-\frac{\delta t}{\delta x}\left(\mathcal{A}^{+} \Delta \phi_{i-\frac{1}{2}}+\mathcal{A}^{-} \Delta \phi_{i+\frac{1}{2}}\right)
$$

- método de primeira ordem de Godunov com termo de correção:

$$
\phi_{i}^{n+1}=\phi_{i}^{n}-\frac{\delta t}{\delta x}\left(\mathcal{A}^{+} \Delta \phi_{i-\frac{1}{2}}+\mathcal{A}^{-} \Delta \phi_{i+\frac{1}{2}}\right)-\frac{\delta t}{\delta x}\left(\tilde{F}_{i+\frac{1}{2}}-\tilde{F}_{i-\frac{1}{2}}\right),
$$

em que

$$
\tilde{F}_{i-\frac{1}{2}}=\mathcal{A}^{+} \phi_{i}+\mathcal{A}^{-} \phi_{i-1}+\frac{1}{2}|A|\left(I-\frac{\delta t}{\delta x}|A|\right)\left(\phi_{i}-\phi_{i-1}\right),
$$

com

$$
\phi_{i}-\phi_{i-1}=\sum_{q}^{M} \tilde{\alpha}_{i-\frac{1}{2}} \hat{s}^{q} \quad \text { e } \quad \tilde{\alpha}_{i-\frac{1}{2}}=\alpha_{i-\frac{1}{2}} \psi\left(r_{i-\frac{1}{2}}^{q}\right) .
$$

Ainda, $\alpha=R^{-1} \delta, R$ é a matriz dos autovetores, $\delta=\phi_{i}-\phi_{i-1}, \psi$ são as funções limitadores de fluxo e $r_{i-\frac{1}{2}}^{q}$ é a razão dos gradientes consecutivos definida por

$$
r_{i-\frac{1}{2}}^{q}=\frac{\alpha_{I-\frac{1}{2}}^{q}}{\alpha_{i-\frac{1}{2}}^{q}}, \quad \text { em que } \quad I= \begin{cases}i-1, & \lambda^{q} \leq 0 \\ i+1, & \lambda^{q}>0\end{cases}
$$

com $\lambda^{q}$ autovalores de $R$.

É importante observar que qualquer esquema convectivo pode ser implementado no software CLAWPACK, desde que se obtenha seu limitador de fluxo. Neste trabalho, todos os esquemas de alta resolução apresentados no Capítulo 2 foram implementados neste pacote computacional. 


\section{Referências Bibliográficas}

[1] M. A. Alves, P. J. Oliveira, and F. T. Pinho. A convergent and universally bounded interpolation scheme for the treatment of advection. International Journal for Numerical Methods in Fluids, 41:47-75, 2003.

[2] M. Arora and P.L. Roe. A well-behaved TVD limiter for high-resolution calculations of unsteady flow. Journal of Computational Physics, 132:3, 1997.

[3] B. Ataie-Ashtiani and L. Farhadi. A stable moving-particle semi-implicit method for free surface flows. Fluid Dynamics Research, 38:241-256, 2006.

[4] D. S. Balsara and C. W. Shu. Monotonicity preserving weighted essentially non-oscilatory scheme with increasingly high order of accuracy. Journal of Computational Physics, 160:405-452, 2000.

[5] E. B. Becker, G. F. Carey, and J. T. Oden. Finite Elements - An Introduction. Prentice-Hall, 1981.

[6] T. Bohr, P. Dimon, and V. Piutkaradze. Shallow-water approach to the circular hydraulic jump. Journal of fluid mechanics, 254:635-648, 1993.

[7] A. C. Brandi. Estratégias upwind e modelagem $\kappa-\varepsilon$ para simulação numérica de escoamentos com superfícies livres a altos números de reynolds. Master's thesis, Instituto de Ciências Matemáticas e de Computação - Universidade de São Paulo, 2005.

[8] Y. Brechet and Z. Néda. On the circular hydraulic jump. American Journal of Physics, 67, 1999.

[9] M. Cada and M. Torrilhon. Compact third-order limiter functions for finite volume methods. Journal Computation Physics, 228:4118-4145, 2009. 
[10] A. F. Castelo, M. F. Tomé, C. N. L. César, S. McKee, and J. A. Cuminato. Freeflow: An integrated simulation system for three-dimensional free-surface flows. Journal of Computers and Visualization in Science, 2:199-210, 2000.

[11] A. J. Chorin. Numerical solution of the Navier-Stokes equations. Mathematics Computational, 22:745-762, 1968.

[12] A. Colagrossi and M. Landrini. Numerical simulation of interfacial flows by smoothed particle hydrodynamics. Journal Computation Physics, 191:448-475, 2003.

[13] R. Courant, E. Isaacson, and M. Rees. On the solution of nonlinear hyperbolic differential equations by finite differences. Community Pure Applied Mathematics, 5:243-255, 1952.

[14] J. O. Cruickshank. Low Reynolds number instabilities in stagnating jet flows. Journal of fluid mechanics, 193:111-127, 1987.

[15] R. A. B. de Queiroz and V. G. Ferreira. Development and testing of high-resolution upwind schemes - Upwind schemes for incompressible free surface flows. VDM Verlag Dr Müller, 2010.

[16] P. A. Durbin. On the $\kappa-\varepsilon$ stagnation point anomaly. Journal Heat and Fluid Flow, 17, 1996.

[17] C. Ellegard, A. E. Hansen, A. Haaning, K. Hansen, A. Marcussen, and T. Bohr. Poligonal hydraulic jumps. Nonlinearity, 12:1-7, 1999.

[18] V. G. Ferreira. Análise e implementação de esquemas de convecção e modelos de turbulência para simulação de escoamentos incompressíveis envolvendo superfícies livres móveis. PhD thesis, Instituto de Ciências Matemáticas e de Computação - Universidade de São Paulo, 2001.

[19] V.G. Ferreira, F.A. Kurokawa, R.A.B. Queiroz, M.K. Kaibara, C.M. Oishi, J.A. Cuminato, A. Castelo, M.F. Tomé, and S. Mckee. Assessment of a high-order finite difference upwind scheme for the simulation of convection-diffusion problems. International Journal for Numerical Methods in Fluids, 60:1-26, 2009.

[20] A. O. Fortuna. Técnicas Computacionais para Dinâmica dos Fluidos. 2000.

[21] P. H. Gaskell. and A. K. C. Lau. Curvature-compensated convective transport: SMART, a new boundedness preserving transport algorithm. International Journal for Numerical Methods in Fluids, 8:617-641, 1988.

[22] N. J. Georgiadis, D. P. Rizzetta, and C. Fureby. Large-Eddy Simulation: current capabilities, recommended tractices, and future research. AIAA Journal, 48:1772-1784, 2010. 
[23] F. H. Harlow and E. Welch. Numerical calculation of time-dependent viscous incompressible flow of fluid with free surface. The Physics of Fluids, 8:2182-2189, 1965.

[24] A. Harten. High resolution schemes for hyperbolic conservation laws. Journal of Computational Physics, 49:357-393, 1983.

[25] A. Jameson and L. Martinelli. Mesh refinement and modeling errors in flow simulation. AIAA Journal, 36, 1998.

[26] G. S. Jiang and C. W. Shu. Efficient implementation of weighted ENO shemes. Journal Computation Physics, 126:202-212, 1996.

[27] V. Kumar and S. V. R. Rao. Composite scheme using localized relaxation with non-standard finite difference method for hyperbolic conservation laws. Journal of Sound and Vibration, 311:786-801, 2008.

[28] P. D. Lax and B. Wendroff. Systems of conservation laws. Communications in Pure and Applied Mathematics Computational, 13:217-237, 1960.

[29] B. P. Leonard. The QUICK algorithm: a uniformly third-order finite difference method for highly convective flows. Computational Methods Applicate Mechanic Engineering, 19:59, 1979.

[30] B. P. Leonard. Simple high-accuracy resolution program for convective modelling of discontinuities. International Journal for Numerical Methods in Fluids, 8:1291-1318, 1988.

[31] R. J. LeVeque. Numerical methods for conservation laws. University of Washington, 1992.

[32] R. J. LeVeque. Finite volume methods for hyperbolic problems. University of Washington, 2002.

[33] R. J. LeVeque. Finite Difference Methods for Ordinary and Partial Differential Equations, Steady-State and Time-Dependent Problems. SIAM, 2007.

[34] Y. Li. Wave number-entended high-order upwind-based schemes for convective scalar transport. Journal of Computational Phisics, 133:235-255, 1997.

[35] G. A. B. Lima. Desenvolvimento de estratégias de captura de descontinuidades para leis de conservação e problemas relacionados em dinâmica dos fluidos. Master's thesis, Instituto de Ciências Matemáticas e de Computação - ICMC-USP, 2010.

[36] H. Lin and C.C. Chieng. Characteristic-based flux limiters of an essentially third-order flux-splitting method for hyperbolic conservation laws. International Journal for Numerical Methods in Fluids, 13:287-307, 1991. 
[37] B. Liu, G.R. Lam, K.Y. Song, and R.S. Amano. On a higher-order bounded discretization scheme. International Journal for Numerical Methods in Fluids, 32:881-897, 2000.

[38] D.W. Lomax, H. Zingg, and H. Jurgens. High accuracy finite difference schemes for linear wave propagation. J. Sci. Comput., 17:328-346, 1996.

[39] J. C. Martin and W. J. Moyce. An experimental study of the collapse of liquid columns on a rigid horizontal plane. Philosophical Transactions of the Royal Society, Mathematical, Phisical \& Engeneering Sciences, 244:312-324, 1952.

[40] J. M. Nóbrega, O. S. Carneiro, F. T. Pinho, G. S. Paulo, M. F. Tomé, A. Castelo, and J.A. Cuminato. The phenomenon of jet buckling: Experimental results and numerical predictions. The Polymer Processing Society 23rd Annual Meeting.

[41] M. B. Oliveira. Freeflow-axi: um ambiente de simulação de escoamentos axissimétricos com superfícies livres. Master's thesis, Instituto de Ciências Matemáticas e de Computação Universidade de São Paulo, 2002.

[42] S. V. Patankar. Numerical heat transfer and fluid flows. Hemisphere Publishing Co., New York, 1980.

[43] R. Peyret and T. D. Taylor. Computational methods for fluid flow. New York, 1983.

[44] G.W. Platzman. An exact integral of complet spectral equation for unsteady one-dimensional flow. The University of Chicago, 1964. Manuscript received.

[45] A. Qamar, N. Hasan, and S. Sanghi. New scheme for the computation of compressible flows. AIA A Journal, 44, 2006.

[46] A. Rai, B. S. Dandapat, and S. Poria. hydraulic jump in generalized-Newtonian fluids. arXiv:0809.2231v3 [physics.flu-dyn], 2008.

[47] P. L. Roe. Some contribuitions to the modeling of discontinuous flows. Lect. Notes Appl. Math., 22:163-193, 1985.

[48] P. L. Roe. Characteristic-based schemes for the euler equations. Annual Reviews Fluid Mechanic, 18:337-365, 1986.

[49] B. R. Shin, T. Hikohagi, and H. Daiguji. A modified QUICK scheme with good stability and high convergence rate. Journal of Computational Fluids Dynamics, 7:283-299, 1998.

[50] C. W. Shu and S. Osher. Efficient implementation of essentially non-oscilatory shock capturing schemes. Journal of Computational Physics, 83:32, 1989. 
[51] G. Sod. A survey of several finte difference methods for systems of nonlinear hyperbolic conservation laws. Journal of Computational Phisics, 27:1, 1978.

[52] D. L. Sondak and R. H. Pletcher. Application of wall functions to generalized nonorthogonal curvilinear coordinate systems. AIAA Journal, 33:33-41, 1995.

[53] D. B. Spalding. A novel finite difference formulation for differential expressions involving both first and second derivatives. International Journal for Numerical Methods in Fluids, 4:551-559, 1972 .

[54] P. K. Sweby. High resolution schemes using flux limiters for hyperbolic conservation laws. SIAM Journal on Numerical Analysis, 21:995-1011, 1884.

[55] H. Tang. On the sonic point glitch. Journal Computation Physics, 202:507-532, 2005.

[56] H. Tang and G. Warneckes. A Runge-Kutta discontinuous Galerkin method for the Euler equations. Computers \& Fluids, 2003.

[57] G. I. Taylor. Low Reynolds number flows. National Committee for Fluid Mechanics Films - Illustrated experiments in fluid mechanics, 1974.

[58] M.F. Tomé, A.C. Filho, J.A. Cuminato, N. Mangiavacchi, and S. McKee. GENSMAC3D: a numerical method for solving unsteady three-dimensional free surface flows. International Journal for Numerical Methods in Fluids, 37:747-796, 2001.

[59] E. F. Toro. Riemann Solvers and Numerical Methods for Fluid Dynamics. New York, 1999.

[60] L. N. Trefethen and D. Bau. Numerical Linear Algebra. Society for Industrial and Applied Mathematics, 1997.

[61] B. van Leer. Towards the ultimate conservative difference scheme. II. monotonicity and conservation combined in a second-order scheme. Journal of Computational Phisics, 14, 1974.

[62] B. van Leer. Towards and ultimate conservative difference scheme. IV. a new approach to numerical convection. Journal of Computational Phisics, 23, 1977.

[63] A. Varonos and G. Bergeles. Development and assessament of a variable-order non-oscilatory scheme for convection term discretization. International Journal for $\mathrm{Nu}$ merical Methods in Fluids, 26:1-16, 1988.

[64] L. Ville, L. Silva, and T. Coupez. Convected level set method for the numerical simulation of fluid buckling. International Journal for Numerical Methods in Fluids, 2010. 
[65] N. P. Waterson and H. Deconinck. Design principles for bounded higher-order convection schemes - a unified approach. Journal of Computational Physics, 224:182-207, 2007.

[66] E. J. Watson. The radial spread of a liquid jet over a horizontal plane. Journal of Fluid Mechanics, 20:481-499, 1964.

[67] G.B. Whitham. Linear and nonlinear waves. 1974.

[68] P. Woodward and P. Collela. The numerical simulation of two-dimensional fluid flow with strong shocks. Journal of Computational Phisics, 54:115-173, 1984.

[69] J. Zhu. On the higher-order bounded discretization schemes for finite volume computations of incompressible flows. Computer Methods in Applied Mechanics and Engineering, 98:345-360, 1992.

[70] M. Zijlema. On the construction of a third-order accurate monotone convection scheme with application to turbulent flows in general domains. International Journal for Numerical Methods in Fluids, 22:619-641, 1996. 